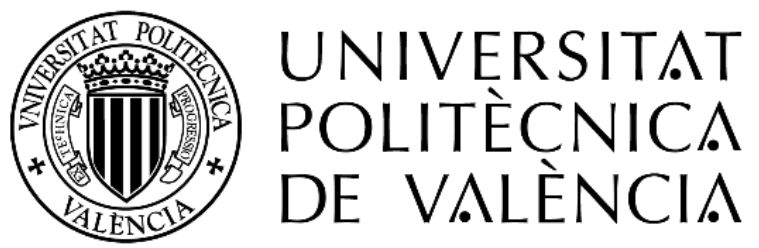

Doctorado en Administración y Dirección de Empresas

\author{
Tesis Doctoral
}

\title{
Modelos de optimización para el diseño estratégico-táctico de una red de transporte intermodal
}

Autora Anny del Mar Agámez Arias

Directores José Pedro García Sabater José Moyano Fuentes

Valencia, septiembre de 2021 


\section{Agradecimientos}

A Dios, siempre me has indicado el camino y me has puesto junto a personas maravillosas; no tengo suficientes palabras escritas para ti Padre.

A mis padres, recuerdo una de sus enseñanzas: cuando empieces algo debes termínalo, y si decides hacerlo que quede bien hecho; más que perfección, es pasión; más que esfuerzo, es entrega; más que éxito, es amor hacia los demás y hacia ti... Su compañía en la distancia y gratitud desde mi corazón.

A mis hermanas, por su apoyo incondicional en cada etapa de mi vida y en ésta por hacerme los días más alegres.

A mis sobrinos, porque me hacen experimentar el amor terrenal más puro que pueda vivir una persona, siempre los extrañé ¡los amo!

A JoséP, por toda la entrega, dedicación y paciencia en este camino, y tu apoyo en las cosas de la vida. Sí, siempre recordaré lo aprendido de ti y a ti. Dios cruza los caminos de las personas con gran ingenio, le agradezco tanto por ello.

A José, por tu disposición, confianza y apoyo en la tesis. Siempre estuviste ahí, en el momento indicado y con las palabras justas. Agradezco a Dios por permitirme conocerte y trabajar contigo.

A Julio Amézquita, por asumir un rol importante en este camino que recorrí, por tantos años de estar trabajando juntos, por los consejos, el cariño y acogerme como una hija. Mi gratitud es inmensa con usted.

A Angel Ruiz, por su paciencia y dedicación en cada reunión, y por todo lo aprendido.

A la Gobernación de Bolívar y Fundación CEIBA (Colombia), por el apoyo financiero para desarrollar ésta tesis doctoral.

A mis demás familiares, ahijados y amigos por mi ausencia durante este tiempo. 


\section{Contenido}

Figuras viii

Tablas $\quad \mathrm{X}$

Resumen $\quad$ xii

Abstract xiv

Resum xvi

Siglas $\quad$ xviii

Capítulo 1. Introducción general ............................................1

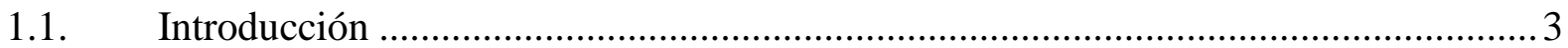

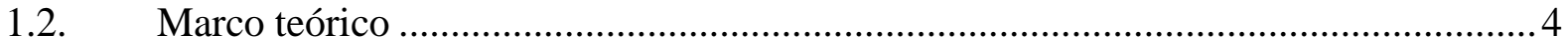

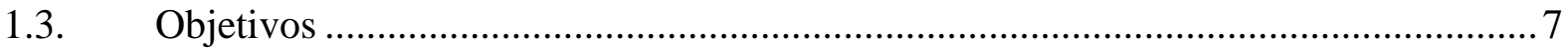

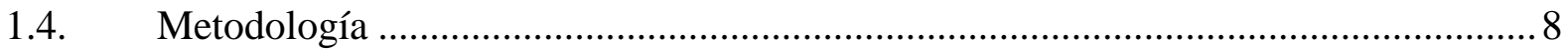

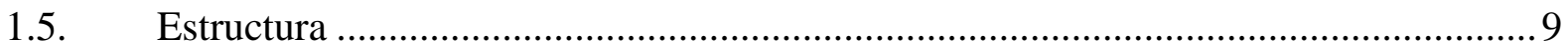

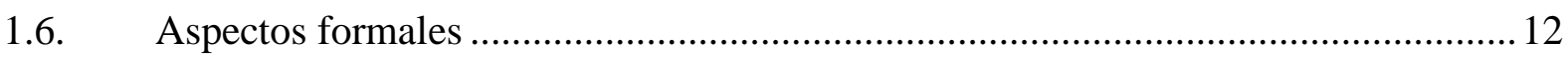

Capítulo 2. Contextualización del problema de estudio: ZODES Magdalena Medio (Bolívar, Colombia) ......................................14

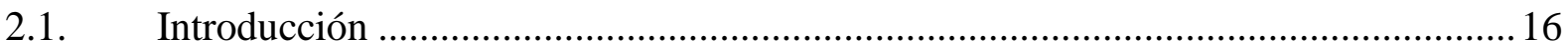

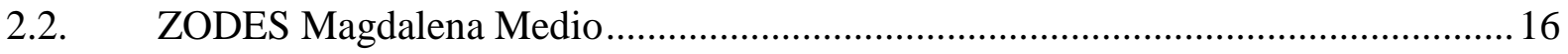

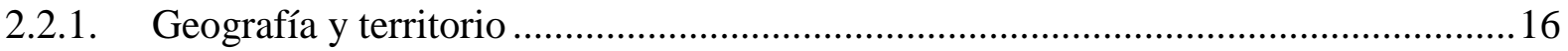

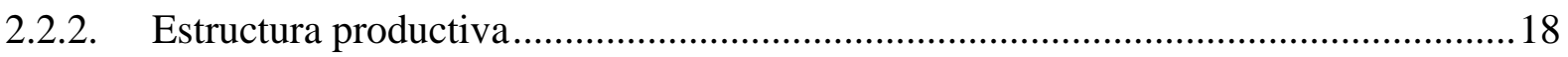

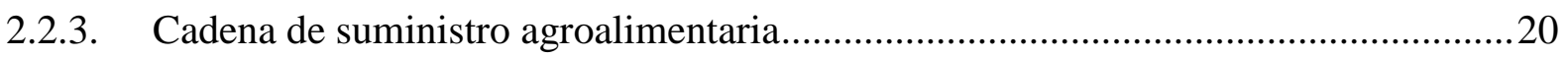

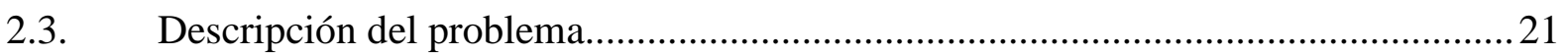

2.3.1. Comercialización y gestión del transporte................................................................22

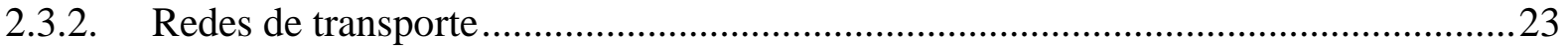

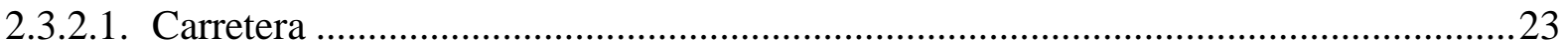

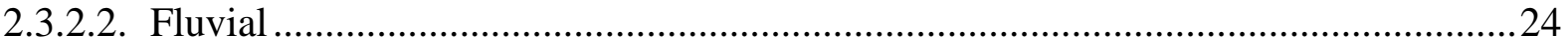

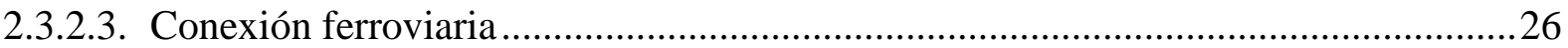

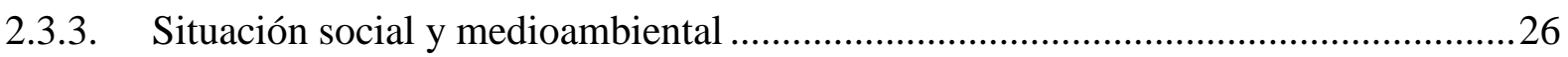

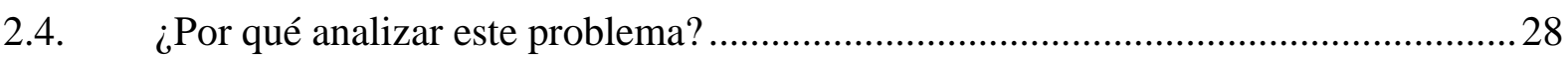

Capítulo 3. Enfoque metodológico: programación matemática ....31

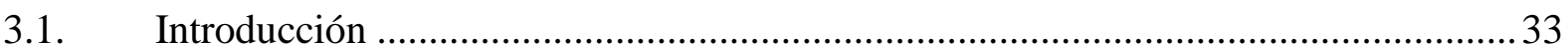

3.2. Técnica de análisis de decisiones: Programación matemática ...................................33

3.3. Problema combinado LI-DR intermodal: Versión simple del problema real ............35

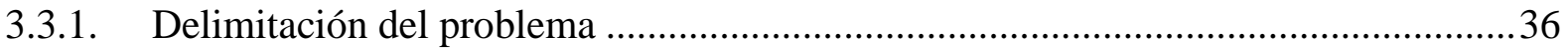

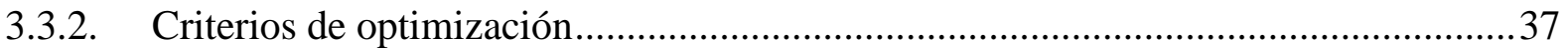


3.3.2.1. Criterios económicos

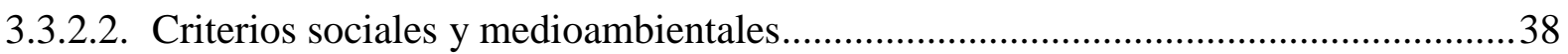

3.4. Modelado del problema combinado LI-DR intermodal para construir los modelos de programación matemática

3.4.1. Modelado de la versión simple del problema ........................................................ 40

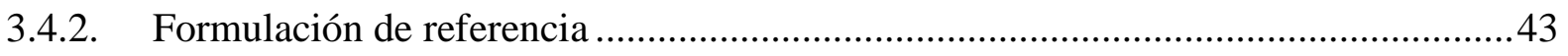

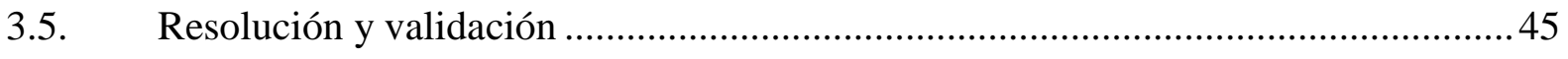

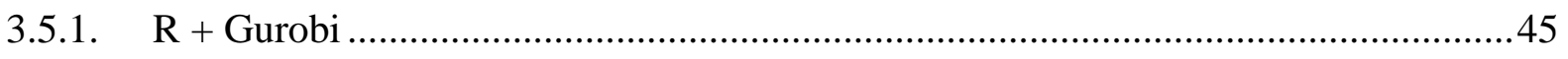

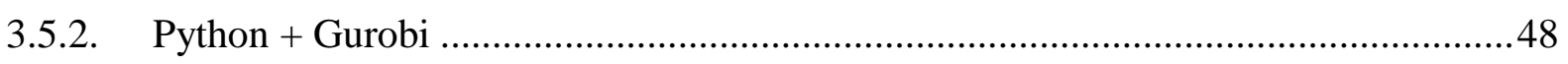

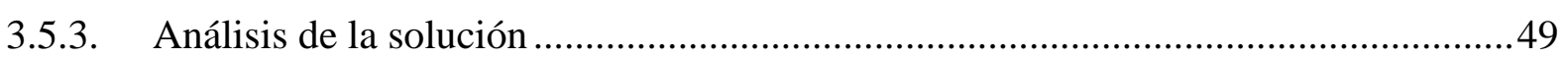

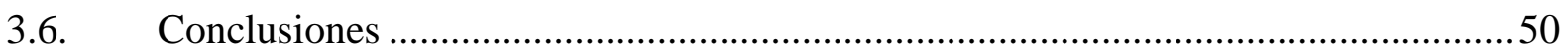

Capítulo 4. Intermodalidad y problemas combinados de localización de instalaciones-diseño de red: estado del arte.......................52

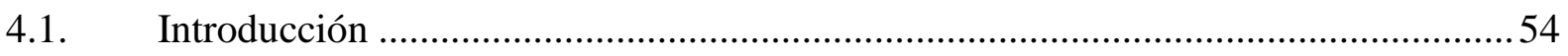

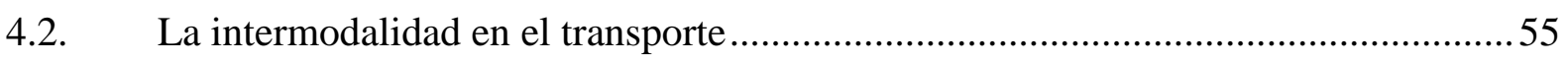

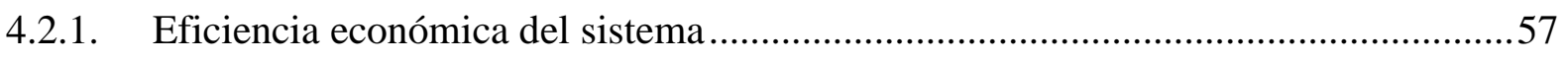

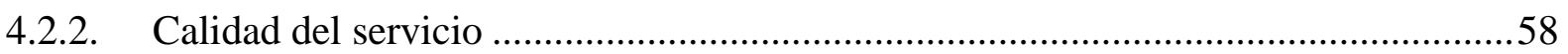

4.3. El transporte intermodal y la planificación de las decisiones .................................59

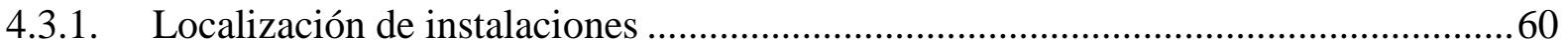

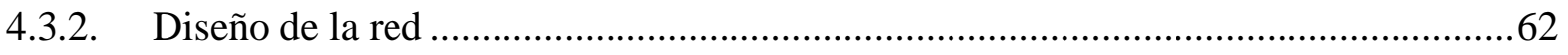

4.3.3. Combinación de los problemas de localización de instalaciones y el diseño de la red 63

4.4. Optimización de problemas combinados LI-DR intermodal: Una RSL-2EV ...........63

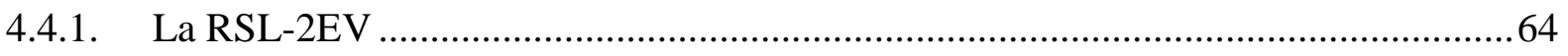

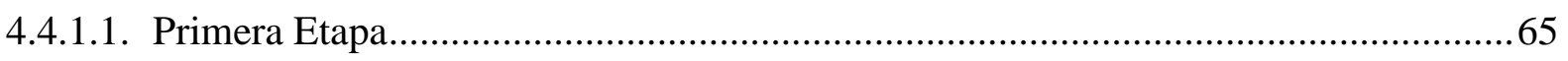

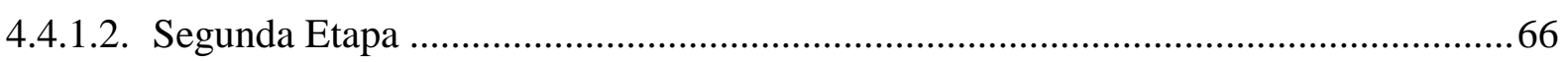

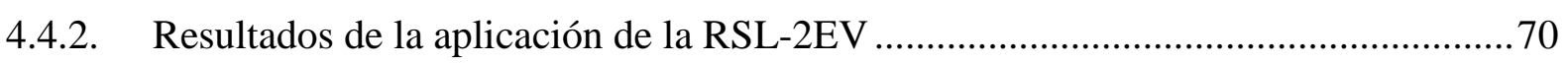

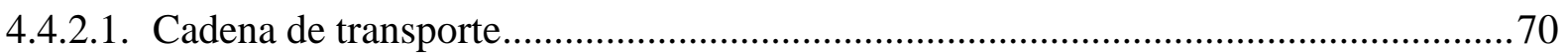

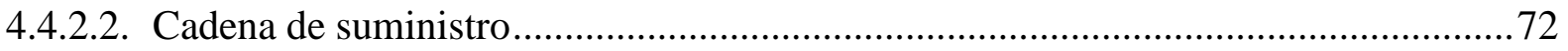

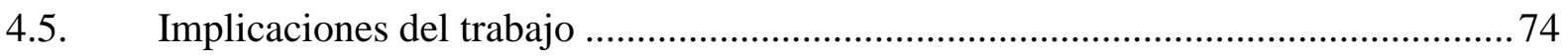

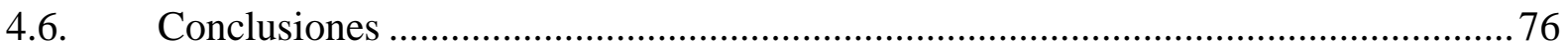

Capítulo 5. Diseño económico de la red de transporte intermodal: propuesta de un modelo de programación lineal entero-mixto

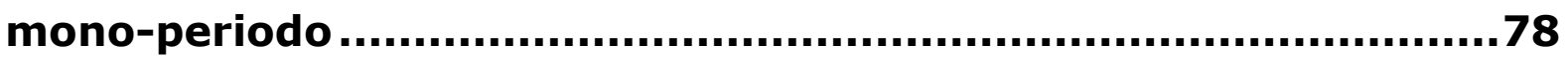

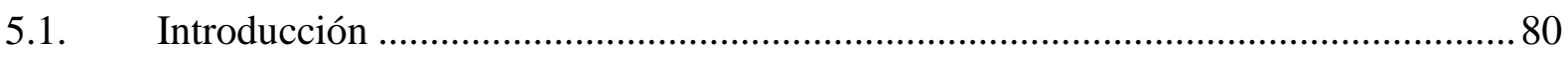

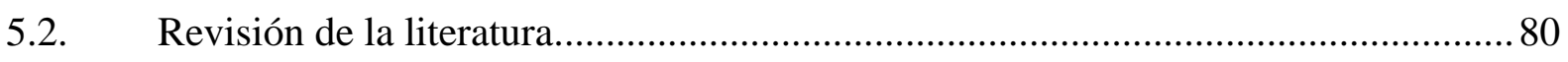

5.2.1. Planificación endógena de la capacidad en las instalaciones ................................... 81 
5.2.2. Limitación del presupuesto de inversión .82

5.3. Propuesta del MPLEM mono-periodo con enfoque económico ............................... 83

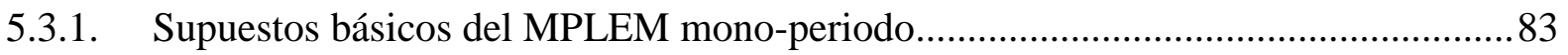

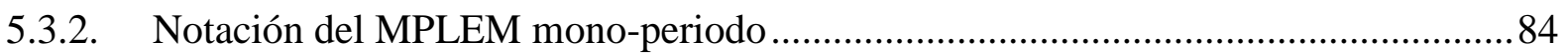

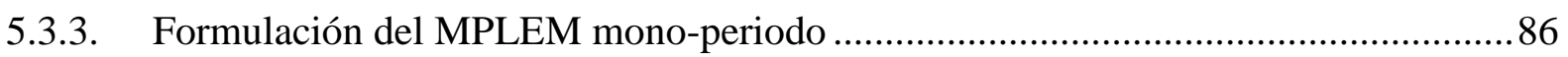

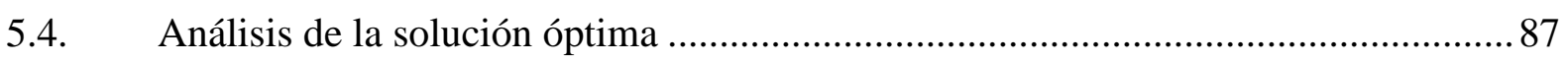

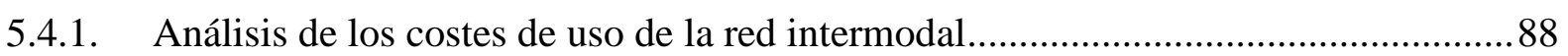

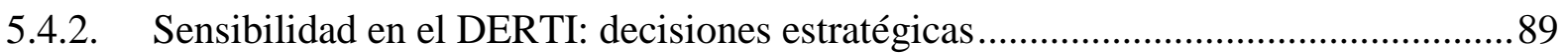

5.4.3. Sensibilidad en el DERTI: decisiones tácticas ......................................................99

5.4.3.1. Uso de la capacidad intermodal de las instalaciones ..............................................99

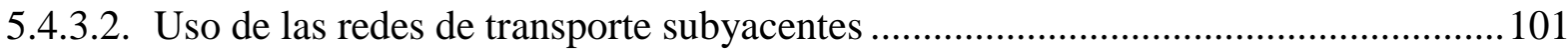

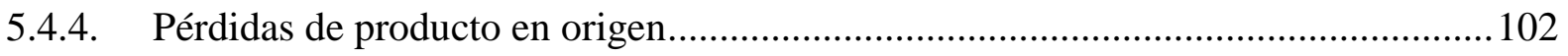

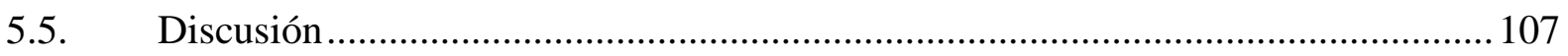

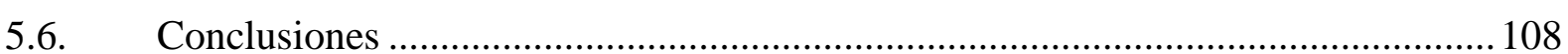

Capítulo 6. Una programación multi-periodo para el análisis de la interdependencia entre los proyectos de inversión y las múltiples fuentes de financiación en el diseño economico de la red intermodal 110

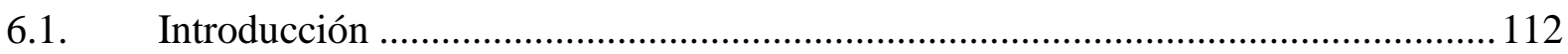

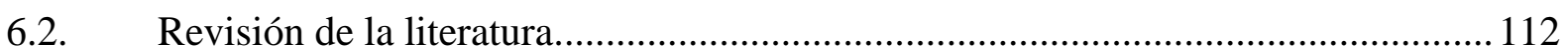

6.2.1. La interdependencia entre los proyectos de inversión ........................................... 113

6.2.2. Múltiples fuentes de financiación: limitación del presupuesto de inversión ............114

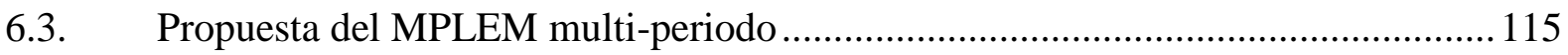

6.3.1. Ajuste al problema de investigación y los supuestos básicos .................................115

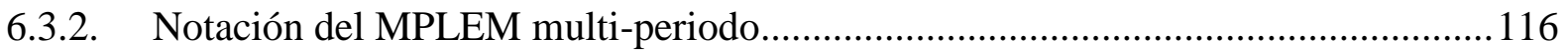

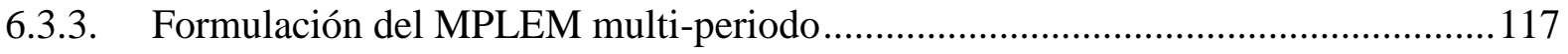

6.4. Priorización de las decisiones de inversión............................................................ 118

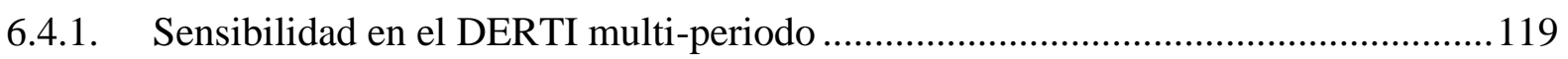

6.4.2. Interdependencia en el diseño multi-periodo de la red intermodal.......................... 126

6.5. Priorización con múltiples presupuestos de inversión de múltiples actores ............132

6.5.1. Efectos en la solución según el presupuesto de inversión de cada actor ...................134

6.5.1.1. Actores y diseño de la red intermodal ...................................................................... 136

6.5.2. Efectos en la solución según el coste social .......................................................... 139

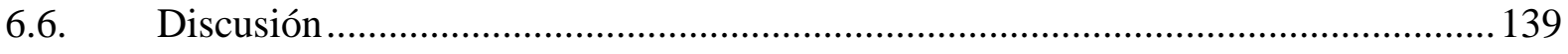

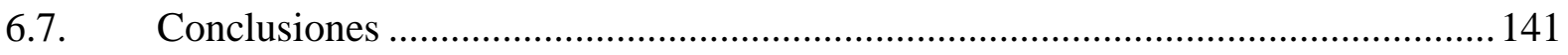




\section{Capítulo 7. Diseño sostenible de la red de transporte intermodal: conflicto entre múltiples objetivos y preferencias de los decisores}

143

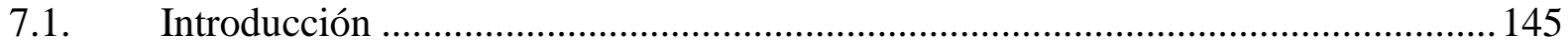

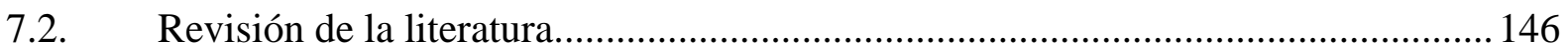

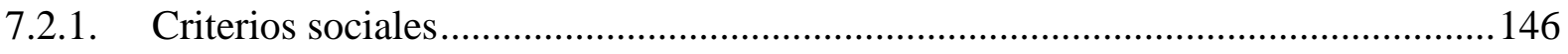

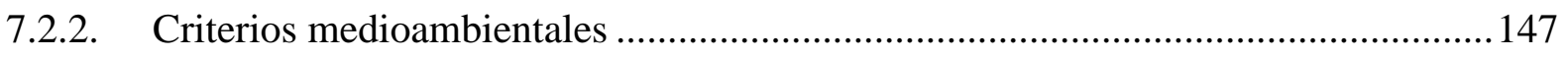

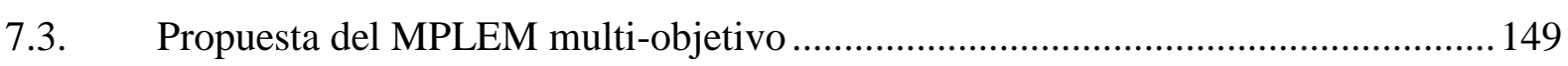

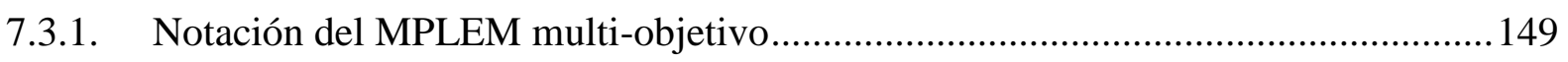

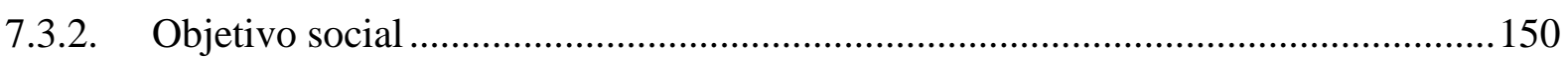

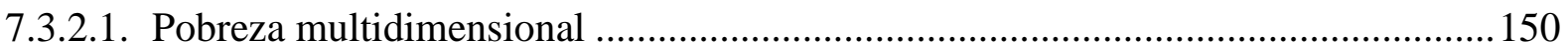

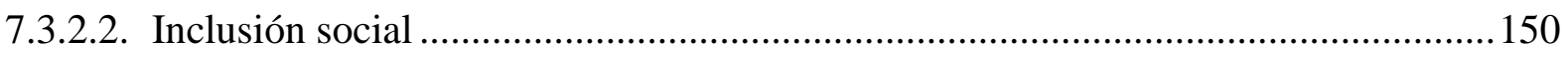

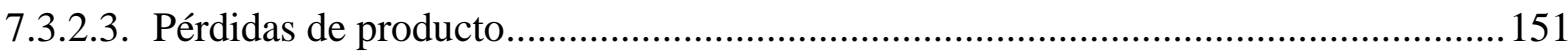

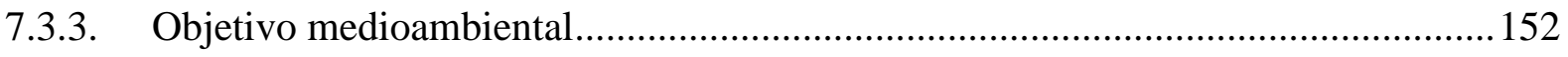

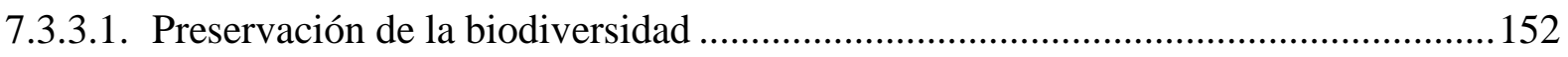

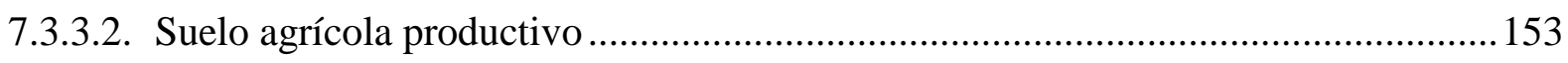

7.4. Optimización individual de las funciones objetivo social y medioambiental..........153

7.4.1. Análisis de la solución óptima social y medioambiental..........................................153

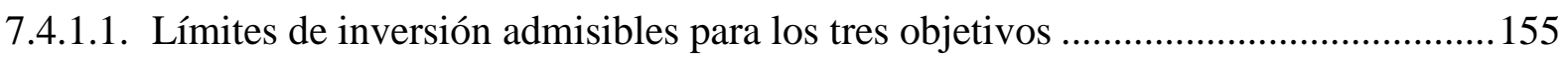

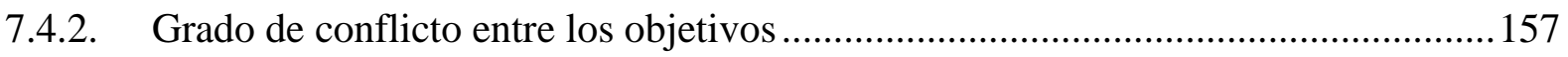

7.5. DSRTI para el problema combinado LI-DR intermodal......................................... 162

7.5.1. Estrategias de Preferencias: Matriz de pago y frontera de Pareto ............................. 162

7.5.2. Soluciones Pareto-óptimas del MPLEM multi-objetivo............................................. 164

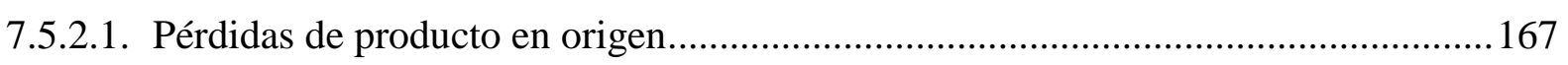

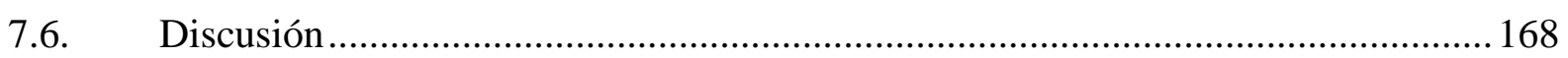

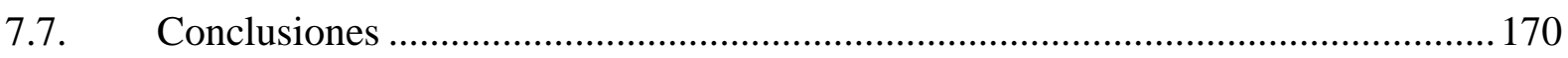

Capítulo 8. Conclusiones generales …..................................171

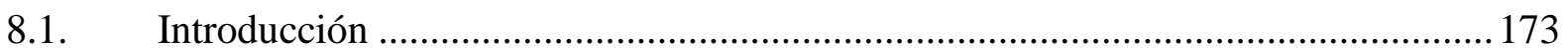

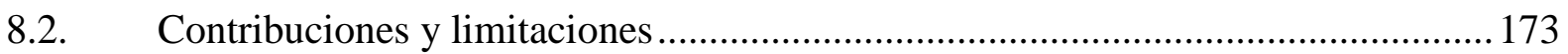

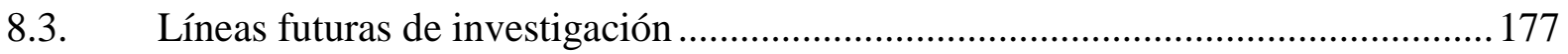

8.4. Implicaciones gerenciales: Administraciones públicas ............................................ 179

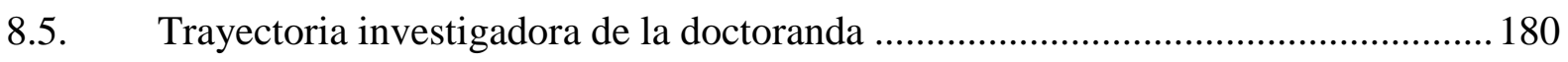

Referencias 185

$\begin{array}{ll}\text { Anexos } & 200\end{array}$ 


\section{Figuras}

Figura 1.1. Problemas de toma de decisiones según el nivel de planificación y decisor...........5

Figura 1.2. Representación de soluciones a un problema combinado LI-DR intermodal .........6

Figura 1.3. Representación gráfica del marco teórico de la tesis............................................

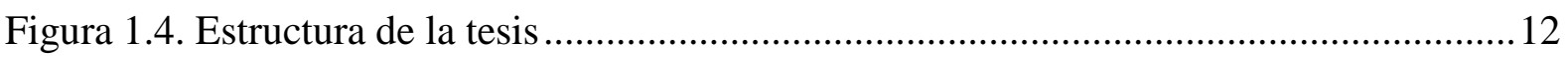

Figura 2.1. Ubicación del departamento de Bolívar y la ZODES Magdalena Medio ............. 17

Figura 2.2. Características geográficas del territorio ........................................................... 18

Figura 2.3. Área sembrada por municipio de la ZODES Magdalena Medio, años 2010 a 2018

Figura 2.4. Producción por municipio de la ZODES Magdalena Medio, años 2010 a 2018 ..20

Figura 2.5. Representación genérica de la cadena de suministro agroalimentaria de la ZODES

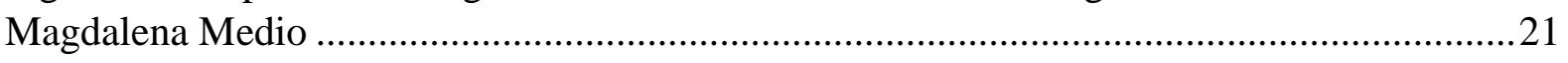

Figura 2.6. Red de carreteras del departamento de Bolívar y proyectos vigentes en la ZODES

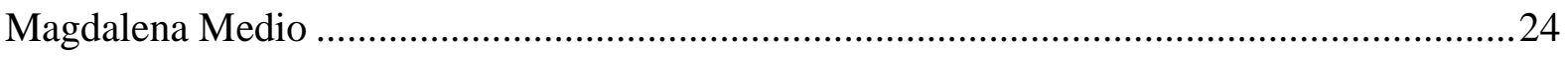

Figura 2.7. Red fluvial del departamento de Bolívar, puertos marítimos y fluviales, y corredores

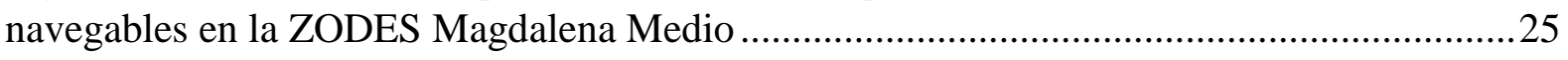

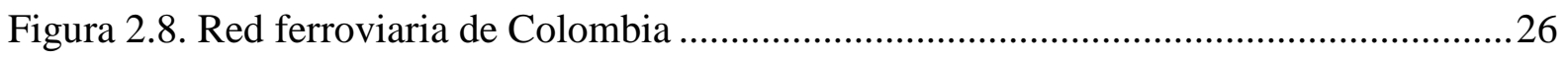

Figura 2.9. Cambios en la cobertura de bosques naturales en el departamento de Bolívar.....28

Figura 3.1. Sistemática para la aplicación de las técnicas de programación matemática.........35

Figura 3.2. Criterios seleccionados para optimizar la solución del problema ..........................38

Figura 3.3. Versiones y variantes de los modelos de programación propuestos en esta tesis

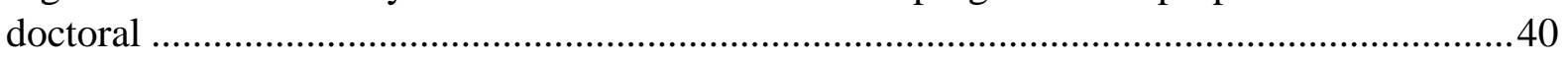

Figura 3.4. Red de entrada para el problema combinado LI-DR intermodal ..........................42

Figura 3.5. Esquema construcción de la Matriz A ................................................................. 47

Figura 3.6. Submatriz de A con entradas no nulas para las variantes de los modelos de

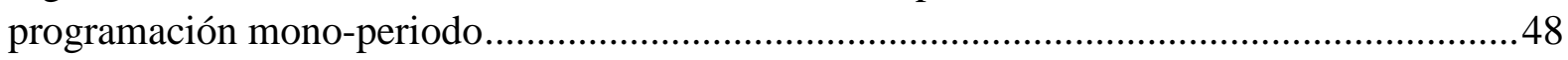

Figura 4.1. Aspectos genéricos de los modelos de programación matemática........................60

Figura 4.2. Etapas y pasos de la RSL-2EV aplicada en los problemas combinados LI-DR ...64

Figura 5.1. Modelado mono-periodo de las alternativas de capacidad/vial de los proyectos de

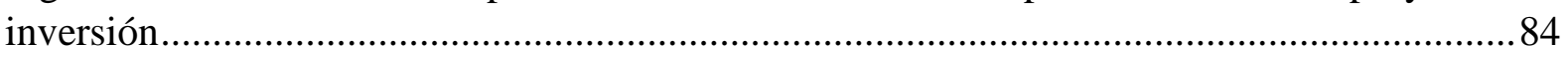

Figura 5.2. Soluciones óptimas en los costes de uso de la red con Cap1 ...............................89

Figura 5.3. Soluciones óptimas en los costes de uso de la red con Cap2 ...............................89

Figura 5.4. Decisiones estratégicas en las soluciones de DERTI .........................................97

Figura 5.5. Uso de la capacidad instalada en las terminales intermodales con Dem1 ........... 100

Figura 5.6. Uso de la capacidad instalada en las terminales intermodales con Cap2 ............101 
Figura 5.7. Uso de las redes de transporte subyacentes.

Figura 5.8. Porcentaje de pérdidas de producto en los nodos de origen con Cap1

Figura 5.9. Porcentaje de pérdidas de producto en los nodos de origen con Cap2.

Figura 5.10. Decisiones estratégicas en las soluciones de DERTI con restricción de pérdidas de producto 106

Figura 6.1. Modelado multi-periodo de las alternativas de capacidad/vial de los proyectos de inversión. 116

Figura 6.2. Decisiones estratégicas en las soluciones de DERTI multi-periodo 124

Figura 6.3. Decisiones tácticas en las soluciones de DERTI multi-periodo 125

Figura 6.4. Proyectos priorizados sin y con interdependencia en las soluciones DERTI multiperiodo 128

Figura 6.5. Proyectos priorizados en las soluciones DERTI multi-periodo con interdependencia para los presupuestos de inversión B1 a B8. 131

Figura 6.6. Valores normalizados del coste de uso de la red para el MPLEM multi-periodo con interdependencias y con múltiples presupuestos de inversión. 136

Figura 6.7. Decisiones estratégicas en las soluciones de DERTI con interdependencias y con múltiples presupuestos de inversión 138

Figura 6.8. Valores normalizados de la solución óptima para distintos valores de costes social 139

Figura 7.1. Soluciones óptimas en el beneficio social con Cap2 154

Figura 7.2. Soluciones óptimas en el impacto medioambiental con Cap2 155

Figura 7.3. Límites de inversión y óptimos admisibles para los objetivos económico y social 156

Figura 7.4. Límites de inversión y óptimos admisibles para los objetivos económico y medioambiental

Figura 7.5. Límites de inversión y óptimos admisibles para los objetivos medioambiental y social 157

Figura 7.6. Grado de conflicto entre los objetivos con presupuesto P12. 160

Figura 7.7. Grado de conflicto entre los objetivos con presupuesto P25. 161

Figura 7.8. Frontera de Pareto para P12, P25, P45 y P59 164

Figura 7.9. Soluciones estratégicas de DSRTI para distintos vectores de peso y presupuesto 167

Figura 7.10. Porcentaje de pérdidas de producto en los nodos de origen con P12 ................ 168

Figura 7.11. Porcentaje de pérdidas de producto en los nodos de origen con P25 168 


\section{Tablas}

Tabla 2.1. Datos de la cadena de suministro agroalimentaria de la ZODES Magdalena Medio

Tabla 2.2. Resultados de los pilares e indicadores del IDC del departamento de Bolívar, año 2020

Tabla 3.1. Decisiones del problema combinado LI-DR intermodal 41

Tabla 3.2. Estructura de la red de entrada del problema combinado LI-DR intermodal..... .43

Tabla 3.3. Nomenclatura utilizada por Amaral et al. (2012) para representar la formulación matemática.....

Tabla 3.4. Variables de decisión y restricciones generadas en las variantes multi-periodo de los modelos de programación

Tabla 4.1. Transporte intermodal y términos relacionados .56

Tabla 4.2. Clasificación de los problemas de decisión por nivel de planificación y actor de la red intermodal .59

Tabla 4.3. Características clave para analizar los estudios seleccionados en la RSL-2EV .....67

Tabla 4.4. Resultados de la RSL-2EV con enfoque en la cadena de transporte .......................72

Tabla 4.5. Resultados de la RSL-2EV con enfoque en la cadena de suministro .....................74

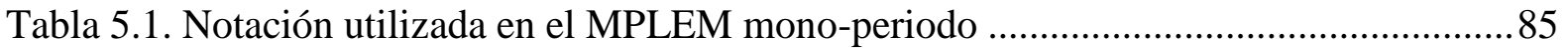

Tabla 5.2. Valores de los parámetros del MPLEM mono-periodo ........................................... 87

Tabla 6.1. Notación complementaria para el MPLEM multi-periodo ................................... 116

Tabla 6.2. Porcentajes del presupuesto de inversión para el MPLEM multi-periodo ........... 119

Tabla 6.3. Valores de los costes para el MPLEM multi-periodo..........................................119

Tabla 6.4. Valores aplicados a los escenarios generados para el MPLEM multi-periodo..... 120

Tabla 6.5. Interdependencia entre los dos tipos de proyectos de inversión ............................ 127

Tabla 6.6. Porcentaje de descuento aplicable por alternativa priorizada ............................... 127

Tabla 6.7. Porcentajes del presupuesto de inversión para el MPLEM multi-periodo con

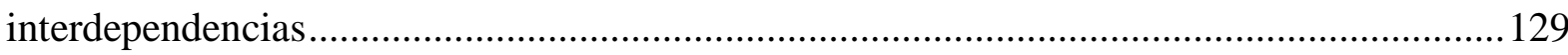

Tabla 6.8. Notación utilizada en el MPLEM multi-periodo con interdependencias y con múltiples presupuestos de inversión

Tabla 6.9. Porcentaje de descuento aplicado a cada alternativa priorizada según cada actor 134

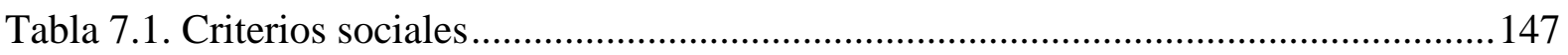

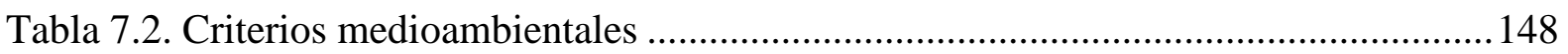

Tabla 7.3. Notación complementaria para el MPLEM multi-objetivo .................................. 149

Tabla 7.4. Requerimientos de inversión por objetivo de optimización con Cap2 ..................155

Tabla 7.5. Matrices de pago normalizadas para P12, P25, P45 y P59 ................................. 163 


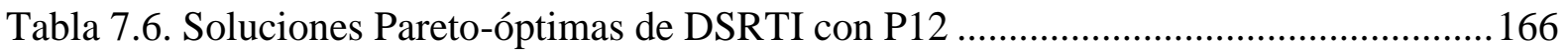

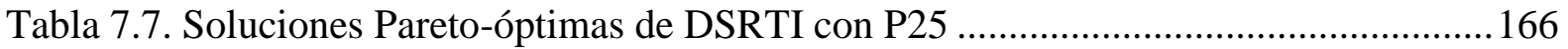




\section{Resumen}

En esta tesis doctoral se desarrollan modelos de programación matemática para el diseño estratégico-táctico de una red de transporte intermodal que combina dos tipos de problemas de decisiones: la localización de instalaciones y el diseño de la red de transporte. Esta combinación se reconoce en la literatura como problemas combinados LI-DR. El problema combinado se estudia para una situación real y se analizan el comportamiento de la solución óptima, a partir de distintos aspectos como: la disponibilidad del presupuesto de inversión, capacidad de las instalaciones intermodales, múltiples periodos de decisiones, interdependencia en la priorización de las decisiones, múltiples fuentes de financiación y criterios de optimización para las tres dimensiones de sostenibilidad. Para la situación real se referencian las condiciones de acceso y conectividad de la Zona de Desarrollo Económico y Social (ZODES) Magdalena Medio del departamento de Bolívar en Colombia, y el potencial de la industria agroalimentaria para esta subregión.

Con el propósito de identificar factores clave que perfilen la formulación respecto a la composición y funcionamiento de los sistemas intermodales y en los problemas combinados LI-DR se analiza la literatura desde estas dos perspectivas. El análisis de la literatura ha permitido aportar dos clasificaciones novedosas e identificar retos para la investigación futura.

Para la formulación de los modelos se lleva a cabo la sistemática de pasos definidos para la aplicación de las técnicas de programación matemática. Con estos pasos se logra transformar el problema del mundo real a un problema manejable con estas técnicas. La transformación favorece la interpretación matemática del problema combinado LI-DR intermodal, la modelación de los datos y la definición de una estructura de red de entrada para indexar las decisiones estratégicas y tácticas. Los modelos de programación matemática se construyen de manera gradual. En concreto, se proponen 2 versiones que se representan en 5 variantes.

Se comienza formulando un modelo de programación lineal entero-mixto (MPLEM) monoperiodo para analizar, desde un enfoque económico, la sensibilidad de las capacidades del sistema intermodal, la capacidad financiera de los tomadores de decisiones y la variación de la demanda. A continuación, sobre la base de este modelo se propone un MPLEM multi-periodo y dos variantes para validar las condiciones de interdependencia en la toma de decisiones estratégica y la participación de múltiples actores en la financiación de los proyectos de inversión. Finalmente, se formula un MPLEM multi-objetivo para optimizar simultáneamente las tres dimensiones de sostenibilidad. Para resolver y validar los modelos se implementaron dos esquemas de resolución. En los esquemas se utilizan los lenguajes de programación R y Python con el software de optimización matemática Gurobi Optimizer. Se realizan experimentos numéricos para distintos escenarios y se analiza el comportamiento de las soluciones considerando distintos valores a los parámetros. Los resultados obtenidos permiten comprobar la utilidad de los modelos matemáticos e identificar las principales limitaciones y futuras líneas de trabajo. 


\section{Palabras clave:}

Problema combinado LI-DR intermodal; Diseño de redes; Intermodalidad; Modelos de optimización; Modelo de programación lineal entero-mixto; Presupuesto de inversión; Interdependencia entre proyectos, Múltiples actores; Fuentes de financiación; Sostenibilidad; Sostenibilidad social, Sostenibilidad medioambiental; ZODES Magdalena Medio. 


\section{Abstract}

In this doctoral thesis, mathematical programming models are developed aiming at the strategic-tactical design of an intermodal transport network that combines two types of decision problems: the location of facilities and the transport network design. In the literature, this combination is recognized as combined LI-DR problems. The combined problem is studied for a real situation and the performance of the optimal solution is analyzed in relation to different aspects such as the investment budget availability, the intermodal facilities capacity, multiple decision periods, interdependence in the prioritization of the decisions, multiple sources of funding and optimization criteria for the three dimensions of sustainability. In what concern to the real situation, the access and connectivity conditions of the Magdalena Medio Economic and Social Development Zone (ZODES, for its acronym in Spanish) of the Bolívar department in Colombia, and the potential of the agri-food industry for this subregion, are considered.

Aiming the identification of key factors that outlines the formulation regarding the composition and operation of intermodal systems and, in combined LI-DR problems, the literature is reviewed from these two perspectives. The literature analysis has made it possible to provide two novel classifications and to identify challenges for future research.

The formulation of models follows the systematic steps already defined for the application of mathematical programming techniques. Following these steps, it is possible to transform the problem from a real-world problem to a manageable one. The transformation promotes the mathematical interpretation of the intermodal LI-DR combined problem, the data modeling, and the definition of an input network structure to index strategic and tactical decisions. Mathematical programming models are built gradually. Specifically, 2 versions are proposed, which are represented by 5 variants.

Firstly, it is formulated a single-period mixed-integer linear programming model (MILPM) in order to analyze, from an economic perspective, the sensitivity of the intermodal system capacities, the financial capacity of the decision makers, and the demand changes. Based on the aforementioned model, a multi-period MILPM and two variants are proposed aiming to validate the conditions of interdependence in strategic decision-making and the participation of multiple actors in the investment projects financing. Finally, a multi-objective MILPM is formulated to simultaneously optimize all three dimensions of sustainability. To solve and validate the models, two resolution schemes were implemented. The schematics use the $\mathrm{R}$ and Python programming languages with the mathematical optimization software Gurobi Optimizer. Numerical tests are carried out for different scenarios and the performance of the solutions is analyzed considering different values for the parameters. The results obtained allow us to verify the usefulness of the models proposed and identify the main limitations and future lines of work. 


\section{Keywords:}

Intermodal FL-ND problem; Network design; Intermodality; Optimization models; Mixedinteger linear programming model; Investment budget; Interdependence between projects, Multiple actors; Sources of funding; Sustainability; Social sustainability, Environmental sustainability; ZODES Magdalena Medio. 


\section{Resum}

En aquesta tesi doctoral es desenvolupen models de programació matemàtica per al disseny estratègic-tàctic d'una xarxa de transport intermodal que combina dos tipus de problemes de decisions: la localització d'instal-lacions i el disseny de la xarxa de transport. Aquesta combinació es reconeix en la literatura com problemes combinats LI-DR. El problema combinat s'estudia per a una situació real i s'analitzen el comportament de la solució òptima, a partir de diferents aspectes com: la disponibilitat de l'pressupost d'inversió, capacitat de les instal·lacions intermodals, múltiples períodes de decisions, interdependència en la priorització de les decisions, múltiples fonts de finançament i criteris d'optimització per a les tres dimensions de sostenibilitat. Per a la situació real es referencien les condicions d'accés i connectivitat de la Zona de Desenvolupament Econòmic i Social (ZODES) Magdalena Medio de el departament de Bolívar a Colòmbia, i el potencial de la indústria agroalimentària per a aquesta subregió.

Amb el propòsit d'identificar factors clau que perfilin la formulació respecte a la composició i funcionament dels sistemes intermodals i en els problemes combinats LI-DR s'analitza la literatura des d'aquestes dues perspectives. L'anàlisi de la literatura ha permès aportar dues classificacions noves i identificar reptes per a la investigació futura.

Per a la formulació dels models es porta a terme la sistemàtica de passos definits per l'aplicació de les tècniques de programació matemàtica. Amb aquests passos s'aconsegueix transformar el problema de l'món real a un problema manejable amb aquestes tècniques. La transformació afavoreix la interpretació matemàtica de el problema combinat LI-DR intermodal, la modelació de les dades i la definició d'una estructura de xarxa d'entrada per indexar les decisions estratègiques i tàctiques. Els models de programació matemàtica es construeixen de manera gradual. En concret, es proposen 2 versions que es representen en 5 variants.

Es comença formulant un model de programació lineal sencer-mixt (MPLEM) monoperíode per analitzar, des d'un enfocament econòmic, la sensibilitat de les capacitats de sistema intermodal, la capacitat financera dels prenedors de decisions i la variació de la demanda. A continuació, sobre la base d'aquest model es proposa un MPLEM multi-període i dues variants per validar les condicions d'interdependència en la presa de decisions estratègica i la participació de múltiples actors en el finançament dels projectes d'inversió. Finalment, es formula un MPLEM multi-objectiu per optimitzar simultàniament les tres dimensions de sostenibilitat. Per resoldre i validar els models es van implementar dos esquemes de resolució. En els esquemes s'utilitzen els llenguatges de programació $\mathrm{R}$ i Python amb el programari d'optimització matemàtica Gurobi Optimizer. Es realitzen experiments numèrics per a diferents escenaris i s'analitza el comportament de les solucions considerant diferents valors als paràmetres. Els resultats obtinguts permeten comprovar la utilitat dels models matemàtics i identificar les principals limitacions i futures línies de treball. 


\section{Paraules clau:}

Problema combinat LI-DR intermodal; Disseny de xarxes; intermodalitat; Models d'optimització; Model de programació lineal sencer-mixt; Pressupost d'inversió; Interdependència entre projectes, Múltiples actors; Fonts de finançament; Sostenibilitat; Sostenibilitat social, Sostenibilitat mediambiental; ZODES Magdalena Medio. 


\section{Siglas}

AGRONET Red de Información y Comunicación del Sector Agropecuario de Colombia

ANI Agencia Nacional de Infraestructuras

CORMAGDALENA Corporación Autónoma Regional del Río Grande de la Magdalena

DERTI Diseño económico de la red de transporte intermodal

DMRTI Diseño medioambiental de la red de transporte intermodal

DNP Departamento Nacional de Planeación

DSORTI Diseño social de la red de transporte intermodal

DSRTI Diseño sostenible de la red de transporte intermodal

GEI Gases de efecto invernadero

IDC Índice departamental de competitividad

INVIAS Instituto Nacional de Vías

IPREG Instituto de Políticas Públicas Regional y de Gobierno

IS Inclusión social

LI-DR Localización de instalaciones-diseño de red

MPLEM Modelo de programación lineal entero-mixto

MSP Método de suma ponderada

ODS Objetivos de desarrollo sostenible

PM Pobreza multidimensional

PB Preservación de la biodiversidad

PP Pérdidas de producto

RSL Revisión sistemática de la literatura

RSL-2EV Revisión sistemática de la literatura en dos etapas vinculadas

SAP Suelo agrícola productivo

UPA Unidades de producción agropecuaria

ZODES Zona de desarrollo económico y social 


$$
\text { CApítulO } 1
$$




\section{Capítulo 1}

\section{Introducción general}

Presentación: en esta tesis doctoral se desarrollan y utilizan modelos de programación matemática para el diseño estratégico-táctico de una red de transporte intermodal que combina dos tipos de problemas de decisiones: la localización de instalaciones y el diseño de la red de transporte. El problema combinado se estudia para una situación real y se analiza el comportamiento de la solución óptima considerando distintos valores a los parámetros, y ciertos aspectos que condicionan el problema original. Estos son: la disponibilidad del presupuesto de inversión, capacidad de las instalaciones intermodales, interdependencia en la priorización de las decisiones, múltiples fuentes de financiación y criterios de sostenibilidad. En este capítulo se expone el marco teórico que sustenta los objetivos planteados y la metodología definida, así como una descripción genérica del contenido de los capítulos desarrollados para este trabajo. 


\subsection{Introducción}

El diseño de redes ha sido y sigue siendo un campo de estudio de creciente interés para los sectores de telecomunicaciones e informática, ingeniería civil y obras públicas, distribución de energía, y la logística y el transporte. Aunque los factores que determinan el diseño de una red se distinguen en el contexto de cada sector, es posible afirmar que todas las redes tienen en común dos elementos característicos en su estructura: los nodos y los arcos. El diseño de la red de transporte ${ }^{1}$ hace referencia a un conjunto de decisiones que se toman para definir la mejor estructura de la red. En los nodos se puede decidir, por ejemplo, la localización, capacidad o servicios en puertos, aeropuertos, centros de distribución o fábricas. En los arcos se toman decisiones sobre la configuración de las redes modales de transporte. Estas redes garantizan el flujo de mercancía y la movilidad de las personas entre orígenes y destinos, por ello son importantes las decisiones relacionadas con la expansión del arco o la frecuencia del servicio. El diseño de la red depende de la viabilidad económica y de la práctica de consolidación para utilizar de manera más eficiente los recursos de transporte (Woxenius, 2007). La consolidación influye, entre otros, en la reducción de los costes unitarios de transporte y en el uso de la capacidad de las instalaciones y los vehículos (Khooban, 2011).

El diseño de la red de transporte es tan importante para la cadena de transporte como para la cadena de suministro y los gobiernos. El desempeño logístico de las empresas que integran estas cadenas se ve comprometido cuando la cantidad transportada, los modos y tiempos de transporte, y la ubicación de las instalaciones/terminales no proporcionan un equilibrio competitivo para programar y coordinar los pedidos e inventarios de acuerdo con los requerimientos de los clientes (Chopra \& Meindl, 2013). Los gobiernos desempeñan un papel fundamental sobre el diseño de la red de transporte, puesto que son los grandes proveedores (financiadores) de las infraestructuras y establecen leyes que regulan esta actividad (Peng et al., 2019). Además, su interés radica en mejorar la eficiencia del transporte para impactar en las economías a través del incremento del comercio, tanto como en reducir efectos negativos en la sociedad y el medioambiente (Ranaiefar \& Amelia, 2011). Desde estas perspectivas el transporte intermodal ofrece mayores ventajas que el transporte efectuado por un único modo (Delbart et al., 2021). El diseño redes de transporte intermodal es más desafiante ya que las capacidades para determinar los flujos de transporte y la infraestructura, disponible y necesaria, se evalúan en conjunto; siendo éstas últimas pilares críticos para el crecimiento de una región o nación (Tripathi et al., 2021).

Esta tesis aborda el diseño de la red de transporte intermodal en un contexto concreto investigando, desde los niveles de decisión estratégico y táctico, los efectos de los factores clave para la construcción y funcionamiento de la red. En este capítulo se exponen los argumentos teóricos que sustentan los objetivos planteados y se presenta una descripción genérica del contenido de los capítulos desarrollados para atender estos objetivos. En la sección 1.2. se definen las bases teóricas y los conceptos clave alrededor de los cuales se fundamenta la tesis. En la sección 1.3. se presentan los objetivos que se persiguen. En la sección 1.4. se describe el enfoque metodológico empleado para desarrollarla. En la sección 1.5. se precisa la

\footnotetext{
${ }^{1}$ El diseño de redes se comprende e interpreta con sutiles diferencias en el sector de la logística y transporte. En el ámbito de la cadena de transporte, se enfatiza en las decisiones de localización y/o expansión de los arcos, y en las decisiones relacionadas con el ajuste de la capacidad en los nodos (Farahani, Miandoabchi, et al., 2013). Por su parte, en el ámbito de la cadena de suministro suelen ser más importantes las decisiones en los nodos que en los arcos, por lo que se simplifican las decisiones en estos últimos hasta definirlas como conexiones factibles entre los nodos sin estar sujetas a la configuración física de las redes de transporte (Badri et al., 2013; Bouzembrak et al., 2013).
} 
estructura adoptada para agrupar los resultados en los distintos capítulos. En la sección 1.6. se especifican algunos aspectos formales para el seguimiento del documento.

\subsection{Marco teórico}

El transporte intermodal hace referencia al uso de dos o más modos de transporte para mover mercancías desde un origen hasta un destino. Para efectuar el movimiento es indispensable, por lo menos, que la mercancía esté contenida en unidades de carga y que se disponga de instalaciones para realizar la consolidación/desconsolidación y transferencia de esta unidad (Crainic \& Kim, 2007) ${ }^{2}$. En estos procesos se involucran distintos tipos de operadores de transporte, operadores de la terminal y operadores de la red cuyo objetivo principal en prestar servicios con eficiencia en costes, considerando mejoras en los tiempos, confiabilidad, flexibilidad del servicio y el impacto al medioambiente, entre otros aspectos (Hasan et al., 2021). Por ello es de significativa importancia la estructura de nodos y arcos de la red de transporte. En las redes intermodales la planificación de estas estructuras ${ }^{3}$ tiene relación con un extenso grupo de problemas de toma de decisiones que pueden llegar a implicar un elevado número de variables y limitaciones.

Una propuesta clásica en la literatura sugiere la agrupación de los problemas según el nivel de planificación y los decisores que participan (Caris et al., 2008). La Figura 1.1. representa esta agrupación en el contexto del transporte intermodal. Algunas de las decisiones de nivel estratégico suelen estar relacionados con la localización de instalaciones, el diseño de la terminal intermodal y la cooperación entre empresas. Generalmente, las decisiones estratégicas se emplean como punto de partida para dar solución a las decisiones de nivel táctico. En este nivel algunas de las decisiones corresponden a la asignación de la demanda, elección de modos de transporte, establecimiento de servicios de consolidación, y la estrategia de precios. El nivel de decisión operativo se ocupa de problemas de programación de recursos de personal, enrutamiento de vehículos y ordenes de carga. De acuerdo con el problema de decisión que se analice pueden, o no, participar los distintos actores que conforman la cadena de transporte. Esto indica que para cada decisor pueden surgir problemas en los tres niveles de planificación ${ }^{4}$.

\footnotetext{
${ }^{2}$ El transporte intermodal es un concepto que también se aplica para la movilidad de personas en las áreas de planificación urbana y transporte público, con el propósito de mejorar la accesibilidad, habitabilidad o seguridad con referencia a las estructuras sociales (Bertolini \& le Clercq, 2003; Carpio-Pinedo, 2021). En éstas redes se refuerza la importancia en la localización y capacidad de instalaciones de transferencia, pues desempeñan un papel clave como generadores de sinergias al ser consideradas "lugares de encuentro" (Hernández \& Monzón, 2016). De igual manera, el comportamiento de los viajes para determinar tipos de flujos y frecuencias en servicios prevalece en estos diseños (Farahani et al., 2013).

${ }^{3}$ Es importante mencionar que en los trabajos de Woxenius (2007), SteadieSeifi et al. (2014) y Dua \& Sinha (2019) se identifican seis topologías de red, sin embargo, algunos se consideran superfluos o estrictamente teóricos cuya aplicación real conduce a diseños topológicos híbridos con fuerte énfasis en las decisiones de nivel operacional. Debido a que el problema que se estudia en esta tesis no integra las mencionadas decisiones de planificación, no se profundizan las bases teóricas en este sentido.

${ }^{4}$ En el capítulo 4 se exponen algunos de estos problemas. Igualmente, se pueden consultar los trabajos de Macharis \& Bontekoning (2004), Caris et al. (2008), SteadieSeifi et al. (2014), Crainic (2000) y Elbert et al. (2020) para obtener información detallada de los tipos de problemas de decisión para cada nivel de planificación y decisor.
} 


\section{Figura 1.1. Problemas de toma de decisiones según el nivel de planificación y decisor}

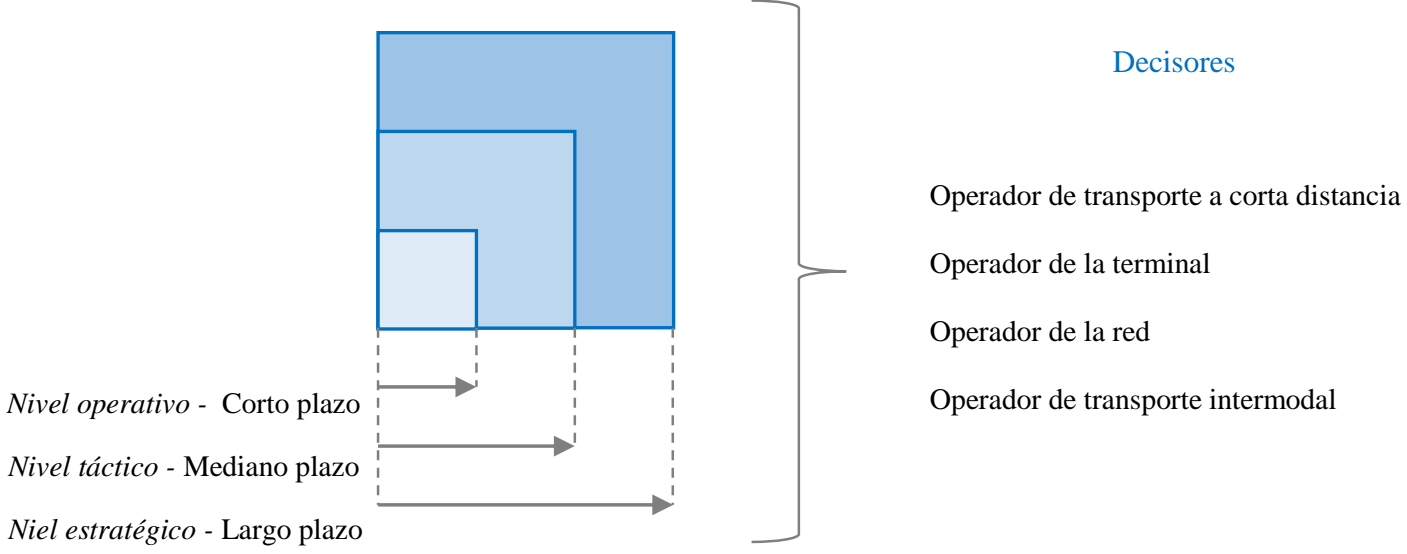

Fuente: Elaboración propia a partir de Caris et al. (2008)

Los problemas de decisión estudiados en la literatura habitualmente se encuentran en uno de los tres niveles de referenciados anteriormente. No obstante, Ortiz-Astorquiza et al. (2018) mencionan que, para aprovechar los efectos en la reciprocidad de las decisiones y acercarse más a la realidad, es conveniente planificar simultáneamente las decisiones de distintos niveles. Algunos de estos problemas son los que combinan las decisiones de localización de instalaciones con la asignación de la demanda (Farahani \& Hekmatfar, 2009), con decisiones de rutas (Schneider \& Drexl, 2017), y con el diseño de la red (Ghaderi \& Jabalameli, 2013). En esta tesis nos enfocamos en los problemas que combinan la localización de instalaciones con el diseño de la red, el cual denominamos en el resto del documento como problemas combinados de localización de instalaciones-diseño de la red (LI-DR). Los problemas combinados LI-DR consideran decisiones estratégicas y tácticas, así como la estructura de las redes de transporte subyacentes (Ortiz-Astorquiza et al., 2018). En estos, básicamente se decide a nivel estratégico donde se localizan los nodos y arcos que diseñan la red, y a nivel táctico, cómo se conectan los nodos y cómo se asigna la demanda a través de los arcos (Sanci \& Daskin, $2019)^{5}$.

La Figura 1.2. representa dos soluciones de un problema combinado LI-DR intermodal. En el problema hay un conjunto de nodos de origen-destino $I$ y $J$, un conjunto de nodos intermodales $K$ en los cuales se ofrecen servicios logísticos, y tres redes modales de transporte que permiten las conexiones modales $S$. Exceptuando los vínculos entre los nodos $K$ de distintas redes modales, las conexiones $S$ se deben entender como una secuencia de arcos de una misma red de transporte. Para identificar una solución se decide la localización de los nodos intermodales $K$ y las conexiones modales $S$ más prometedoras, así como el flujo de carga que se asigna entre los nodos. En la solución 1.2.a. la localización de nodos y conexiones y las decisiones de asignación se concentran principalmente en el modo de transporte 2, mientras que, en la solución 1.2.b. la localización de nodos se equipara entre los modos 1 y 2 , y las conexiones y la asignación se intensifican a través del modo 1. Ambas soluciones tienen un impacto significativo a largo plazo, no obstante, el objetivo de diseño y las limitaciones propias

\footnotetext{
${ }^{5}$ En el capítulo 4 se extiende el alcance de los problemas combinados LI-DR y se presentan los resultados del análisis de la literatura sobre aquellos que son resueltos considerando la intermodalidad.
} 
del problema pueden enmarcar las diferencias en las decisiones ya sea, porque se enfatiza en mejorar los costes, ingresos, tiempos de transporte, distancias, cobertura o servicios (LópezOspina et al., 2021).

Figura 1.2. Representación de soluciones a un problema combinado LI-DR intermodal

a.

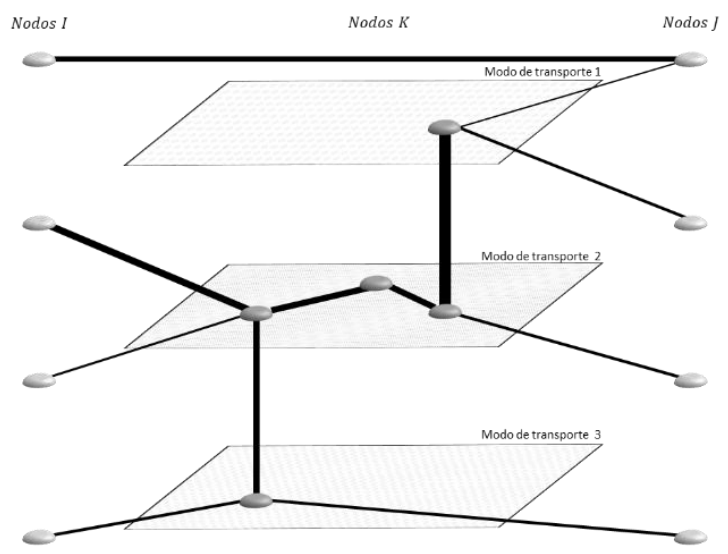

b.

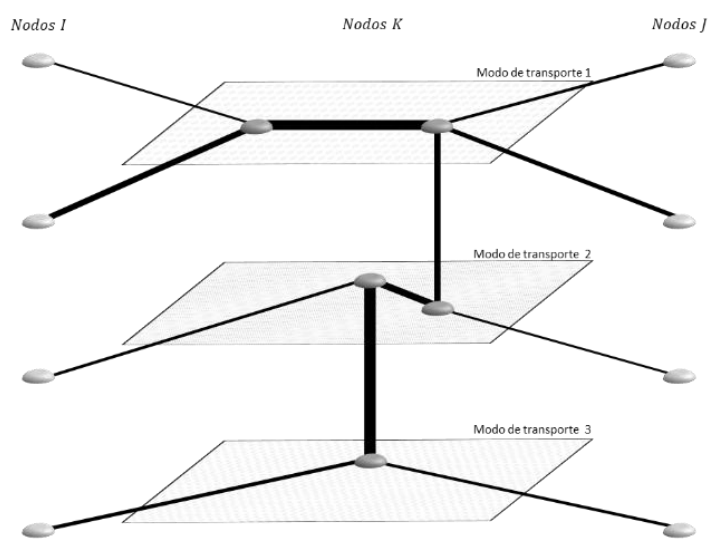

Fuente: Elaboración propia

Las decisiones estratégicas en el diseño de la red requieren, en su mayoría, de grandes inversiones y suelen estar asociadas al desarrollo de políticas generales y funcionales sobre el sistema de transporte (Khooban, 2011). Ante estos requerimientos de inversión se da una participación activa de actores gubernamentales, y según sea el caso de actores privados, que se encargan de la construcción y/o de la administración de las nuevas infraestructuras en la red (Monios \& Wilmsmeier, 2013). Dado que el transporte es uno de los procesos que garantiza la generación de valor agregado en las cadenas de suministro y que la competitividad y flexibilidad global exigen ajustes en la estructura y configuración de la red de suministro, algunos de los actores pertenecientes a esta cadena también se involucran en la inversión de capital para la expansión de las redes de transporte (Hajibabai \& Ouyang, 2013). Además, la construcción y operación de la infraestructura no solo tiene un impacto económico, también provoca cambios en el medioambiente (Broniewicz \& Ogrodnik, 2020) y en la calidad de vida de la población (D’Eusanio et al., 2019).

El impacto económico de estas decisiones ha sido extensamente analizado en la literatura y se relacionan principalmente con mejoras en los costes e ingresos. No obstante, las decisiones de transporte tienen efectos en casi todos los aspectos de la vida humana: movilidad, salud, seguridad, coste de vida, oportunidades económicas y de trabajo, y ocio (Yannis et al., 2020); y efectos en el medioambiente respecto a emisiones de carbono, huella ecológica, contaminantes del aire y el cambio climático, entre otros (Zhao et al., 2020). Es por ello que la toma de decisiones suele ser una tarea profunda y complicada (Camargo-Pérez et al., 2013). Los actores privados tienen intereses de tipo económico, en tanto que, los actores gubernamentales se centran en el impacto social y medioambiental (Anvari \& Turkay, 2017). De igual manera, la participación de los grupos de interés influye en la implementación de la solución, pues sus motivaciones pueden reducir la resistencia o desaciertos propios en la incompatibilidad de intereses para disponer de soluciones sostenibles (Zhao et al., 2020). 
Si bien se reconoce que AHP, ELECTRE, TOPSIS, DEMATEL o PROMETHEE son técnicas de toma de decisiones que han contribuido significativamente en el estudio de los problemas de decisiones en el sector de la logística y transporte (Wątróbski et al., 2019), el segmento de estudios que enfatiza en el transporte intermodal se ha apoyado mayoritariamente en la aplicación de técnicas basadas en modelos analíticos y de simulación (Crainic et al., 2018; Elbert et al., 2020). Estos han sido ampliamente utilizados, por ejemplo, para la localización de terminales intermodales, planificación de la flota de vehículos y diseño de rutas de transporte (Agámez-Arias \& Moyano-Fuentes, 2017). Los modelos analíticos proporcionan un importante conjunto de herramientas para la toma de decisiones, en especial, las técnicas de programación matemática son adecuadas para manejar el nivel de precisión requerido para las soluciones y la complejidad de los problemas que se ocupan del diseño de la red (Arabani \& Farahani, 2012). Además, el diseño de la red con elección del modo de transporte genera interesantes oportunidades para optimizar problemas en el que confluyen diferentes actores con distintos intereses y limitaciones (Caris et al., 2014).

A modo de síntesis respecto a todo lo expuesto en esta sección, se muestra en la Figura 1.3. una representación gráfica del marco teórico que sustenta el desarrollo de la tesis doctoral.

\section{Figura 1.3. Representación gráfica del marco teórico de la tesis}

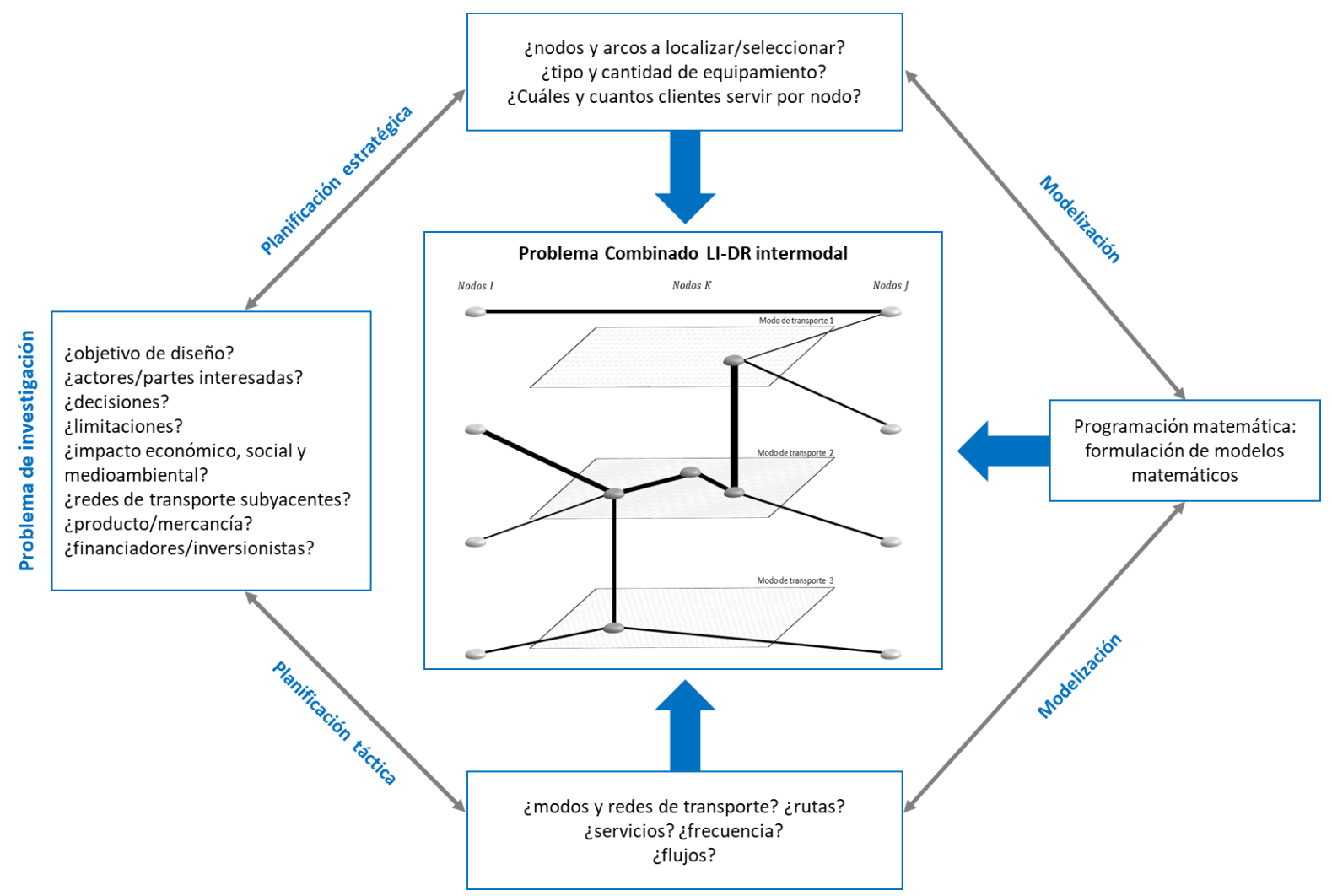

Fuente: Elaboración propia

\subsection{Objetivos}

Esta tesis tiene como objetivo general responder a la siguiente pregunta de investigación: ¿De qué manera los factores clave asociados al funcionamiento del sistema, la calidad del servicio 
y los recursos financieros disponibles influyen en la óptima priorización de proyectos que conforman una red intermodal estratégico-táctica?

Este objetivo general se desglosa en los siguientes objetivos específicos:

$\boldsymbol{O E}-1$ Describir la situación real que ocasiona el problema de transporte y que requiere del diseño de la red intermodal.

$\boldsymbol{O E}-2$ Revisar, clasificar y analizar la literatura para determinar el estado actual de la investigación e identificar oportunidades de trabajo futuro en torno al funcionamiento de los sistemas intermodales y su desempeño logístico para el diseño de la red.

$\boldsymbol{O E}$-3 Simplificar el problema real identificando el tipo y alcance del mismo, e identificar los datos disponibles para el análisis de las posibles soluciones.

$\boldsymbol{O E}-4$ Revisar, clasificar y analizar la literatura para determinar el estado actual de la investigación e identificar oportunidades de trabajo futuro respecto a los problemas combinados LI-DR intermodal.

OE-5 Modelar el problema combinado LI-DR intermodal interpretando la estructura de los datos de la red, los datos del problema y los supuestos básicos.

OE-6 Formular modelos de programación matemática que satisfagan el problema combinado LI-DR intermodal y orienten la toma de decisiones y posibles soluciones para la zona de estudio y la eventual adaptación de la formulación en otros entornos.

$\boldsymbol{O E}-7$ Analizar el comportamiento de la solución óptima explorando el problema combinado LI-DR intermodal a partir de distintos valores para algunos de los parámetros definidos en los modelos de programación.

\subsection{Metodología}

Para lograr los objetivos planteados en la sección anterior la metodología de la investigación se basa en los enfoques interpretativo-exploratorio y experimental. La combinación de los enfoques interpretativo-exploratorio permiten comprender la situación real desde distintas dimensiones para luego describir, fragmentar, categorizar y delimitar el problema de investigación, así como el estado actual de la investigación en el área de conocimiento de interés en esta tesis. Con el enfoque experimental se estudia el problema y analizan los efectos en la solución ante posibles cambios en aspectos particulares del mismo. Los métodos de investigación utilizados son los siguientes:

MI-1 Observación directa no participante, entrevistas no estructuradas y estudio de documentos. Los dos primeros se utilizan para recopilar datos e información de la situación real con el propósito de analizar los diferentes componentes del problema y representar los hallazgos más relevantes de la situación. Con el estudio de documentos oficiales se contrasta la idoneidad y pertinencia de los hallazgos. Estos se utilizan para alcanzar el objetivo específico $O E-1$. 
MI-2 Revisión sistemática de la literatura (RSL). Se utiliza la metodología de los cinco pasos propuestos por Denyer \& Tranfield (2009) para revisar, clasificar y analizar la literatura en relación con el funcionamiento de los sistemas intermodales y el desempeño logístico en el diseño de la red. La RSL se ha aplicado con éxito en otros estudios en el campo del transporte, algunos de estos corresponden a la propuesta de Ginieis et al. (2012), Barbosa-Póvoa et al. (2018), Elbert et al. (2020) y Miraj et al. (2021). Este método se utiliza para alcanzar el objetivo específico $O E-2$.

MI-3 Revisión sistemática de la literatura en dos etapas vinculadas (RSL-2EV). Este método corresponde a una propuesta novedosa para esta tesis y se utiliza en el objetivo específico $O E-4$. La novedad en la propuesta reside en vincular tres nuevos pasos a los cincos establecidos en la RSL de Denyer \& Tranfield (2009) con la finalidad de alinear algunos aspectos indispensables en la revisión, clasificación y análisis de la literatura. Los nuevos pasos se enfocan principalmente en rastrear la literatura en aquellas revisiones previamente publicadas, definir criterios para la localización de los estudios y determinar un esquema de clasificación original para la literatura seleccionada. Con la RSL-2EV se revisa, clasifica y analiza la literatura respecto a los problemas combinados LI-DR intermodal.

MI-4 Modelos analíticos: programación matemática. La programación matemática se utiliza para representar un problema que implica la optimización en un proceso de toma de decisiones (García-Sabater \& Maheut, 2015). Esta procura decidir los mejores valores para los objetivos definidos asignando recursos limitados; tales objetivos representan la meta del o los decisores (Taha, 2012). Siguiendo el conjunto de pasos comúnmente utilizados para desarrollar modelos de programación matemática (Dantzig, 1963; Mathur \& Solow, 1996), se integra esta técnica para el cumplimiento de los objetivos específicos $O E-3, O E-5$ y $O E 6$.

MI-5 Análisis de la solución. Este conduce a analizar los efectos en la solución óptima ante cambios en los valores de los parámetros del modelo de programación matemática. Este método es frecuentemente utilizado en la literatura para respaldar la utilidad de los modelos propuestos y proporcionar directrices a los tomadores de decisiones. En relación con el área de investigación de interés para esta tesis, algunas de las aplicaciones más recientes de este método se pueden referenciar en los trabajos de Badyal et al. (2020), Correia \& Melo (2017), Kumar \& Mishra (2018), Munim \& Haralambides (2018), y Peng et al. (2019). Este análisis se lleva a cabo para alcanzar el objetivo específico $O E-7$.

\subsection{Estructura}

En la Figura 1.4. se muestra la estructura de la tesis. La tesis está organizada en seis capítulos principales, que están precedidos por el presente capítulo de introducción general y sucedidos por el capítulo de conclusiones generales. Los seis capítulos principales se centran en dar respuesta a los objetivos específicos presentados en la sección 1.3. haciendo uso de los métodos mencionados en la sección 1.4. En la Figura se relaciona para cada capítulo los objetivos específicos a los que responden y los métodos de investigación que se utilizan.

En el Capítulo 2, Contextualización del problema de estudio: ZODES Magdalena Medio (Bolívar, Colombia), se presenta una descripción de la situación real que ocasiona el problema de transporte y que requiere del diseño estratégico-táctico de la red de transporte intermodal. La situación toma como referente las condiciones de acceso y conectividad de la ZODES 
Magdalena Medio del departamento de Bolívar en Colombia, y el potencial de la industria agroalimentaria para esta subregión. La interpretación y comprensión del problema permite definir un marco de análisis para desarrollar los modelos de programación matemática.

En el Capítulo 3, Enfoque metodológico: programación matemática, se explica la sistemática llevada a cabo para transformar el problema del mundo real a un problema manejable desde la óptica de la programación matemática, evitando dar paso a la pérdida de representatividad de la situación real. La sistemática, si bien se corresponde con etapas estandarizadas y arraigadas fuertemente entre investigadores y académicos para efectuar tal transformación, soporta su distinción en aspectos que afianzan la calidad en la toma de decisiones y en la aplicabilidad de distintos esquemas de resolución informática. La aplicación de la secuencia de pasos conlleva, primero, a simplificar la situación real identificando el problema combinado LI-DR, para luego, modelarlo y proponer las formulaciones y los esquemas de resolución. La modelación de los datos del problema y la estructura de la red de entrada permiten definir una formulación base para desarrollar los modelos de programación. Los esquemas de resolución se implementan en dos estructuras de codificación: $\mathrm{R}$ y Python. De este modo, el capítulo surte efectos orientativos e introductorios sobre el modelado del problema desde todas sus vertientes, que son fácilmente adaptables en contextos de toma de decisiones similares.

En el Capítulo 4, Intermodalidad y problemas combinados de localización de instalaciones-diseño de red: estado del arte, se revisa sistemáticamente la literatura desde dos perspectivas. En la primera, centrada en las aportaciones alrededor de los sistemas de transporte intermodal, se identificaron los factores clave en la composición y funcionamiento del sistema. En la segunda, la revisión se focalizó en los problemas combinados LI-DR basados en el uso de técnicas de programación matemática. La exploración desde las dos perspectivas se efectúa a razón del elevado número de elementos que distinguen el problema que se trata en esta tesis, y de la posible imprecisión de los hallazgos al pretender la toma de decisiones desde una sola perspectiva. En ambas se proponen clasificaciones novedosas que facilitan la interpretación de la literatura y actualización futura del estado de la misma. En el capítulo se presentan los resultados siguiendo un esquema desde lo general hasta lo particular, destacando especialmente los resultados obtenidos para la segunda perspectiva.

En el Capítulo 5, Diseño económico de la red de transporte intermodal: propuesta de un modelo de programación lineal entero-mixto mono-periodo, se analiza la solución de diseño económico de la red de transporte intermodal (DERTI) que da respuesta al problema combinado LI-DR. Para ello se desarrolla un modelo de programación lineal entero-mixto (MPLEM) mono-periodo que incorpora las lagunas identificadas en la literatura respecto a la planificación endógena de la capacidad del sistema y la importancia de los costes como uno de los factores clave para dar solución al problema. El análisis a la solución óptima, si bien refleja un comportamiento esperado y aceptable, deja al descubierto desequilibrios ocasionados por un aspecto característico del problema: las pérdidas de producto que se ocasionan en los nodos de origen. A partir de este resultado se valida el modelo de programación incorporando una nueva restricción para limitar las pérdidas en estos nodos. Los resultados muestran la utilidad del modelo a la vez que proporcionan información vital para guiar la toma de decisiones de las administraciones públicas. 
En el Capítulo 6, Una programación multi-periodo para el análisis de la interdependencia entre los proyectos de inversión y las múltiples fuentes de financiación en el diseño económico de la red intermodal, se propone la extensión multi-periodo del MPLEM presentado en el capítulo 5. La incorporación del horizonte de planificación en el diseño de la red intermodal conlleva a evaluar la transición en el tiempo de la solución de DERTI. Este modelo se emplea como formulación base para validar dos condiciones del problema que se estudia: la interdependencia entre los proyectos de inversión y los múltiples presupuestos de inversión. En la primera se modela la interdependencia entre los proyectos con el propósito de relacionar los costes de construcción y garantizar el uso de las instalaciones. En la segunda, además de la interdependencia, se modela la participación de múltiples actores como financiadores de los proyectos con la finalidad de analizar los efectos en las decisiones. Se realizan experimentos numéricos para distintos escenarios con el objetivo de explorar los efectos en las decisiones ante incrementos en los diferentes costes, presupuesto de inversión y variaciones en la demanda. Los análisis permiten intuir una jerarquía de decisiones relacionadas con la asignación de los recursos económicos, valorar la posibilidad de desestimar alguna decisión de inversión, y gestionar la cartera de proyectos y participación de inversores privados.

En el Capítulo 7, Diseño sostenible de la red intermodal: conflicto entre múltiples objetivos y preferencias de los decisores, se propone la versión multi-objetivo del MPLEM presentado en el capítulo 5. Esta versión íntegra las tres dimensiones de sostenibilidad identificados para el problema de decisiones: económica, social y medioambiental. El modelo aporta al estado de la cuestión ya que las dimensiones social y medioambiental han sido escasamente exploradas en la literatura del campo del transporte. Las tres dimensiones tratan, en conjunto, de impulsar la actividad económica, el beneficio social y la preservación del patrimonio natural. El análisis a las soluciones Pareto-óptimas se basa en determinar los efectos ante cambios en las preferencias de los decisores para cada objetivo y ante la disponibilidad de financiación como limitante del diseño. Los resultados demuestran que el conjunto de soluciones Pareto-óptimas y el conflicto entre los objetivos varía con el presupuesto de financiación disponible, y que un mismo grupo de preferencias tienen efectos distintos según la disponibilidad de inversión para tomar la decisión.

Finalmente, en el Capítulo 8 , Conclusiones generales, se presentan las principales contribuciones y limitaciones al trabajo realizado, se describen las oportunidades identificadas para el desarrollo de futuras líneas de investigación, se proponen algunas consideraciones para las administraciones públicas y se presenta la trayectoria seguida por la autora durante su etapa formativa doctoral. 
Figura 1.4. Estructura de la tesis

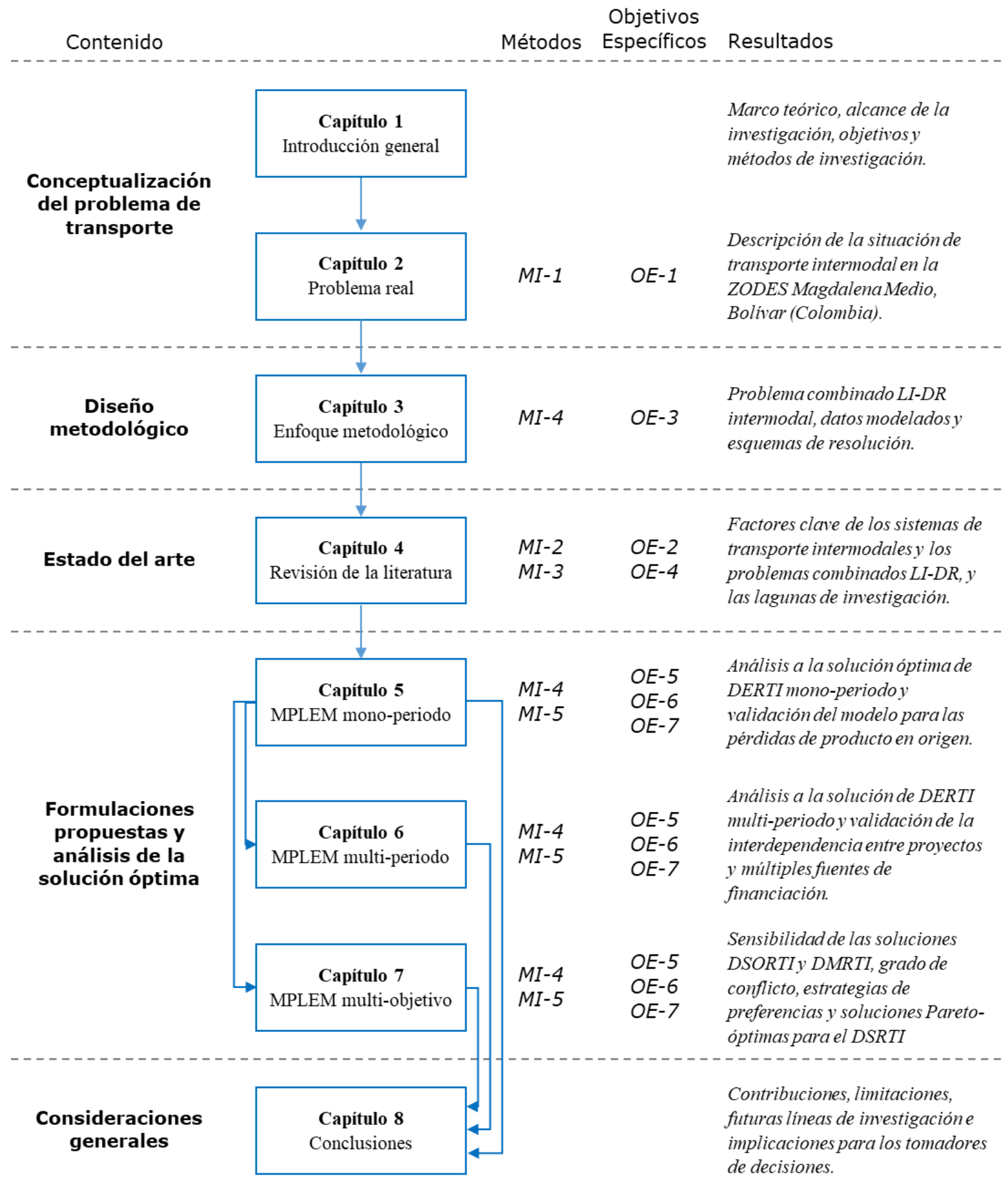

Fuente: Elaboración propia

\subsection{Aspectos formales}

En esta sección se presentan algunos aspectos formales que orientan la lectura y seguimiento de este documento.

- Los capítulos 2, 3 y 4 tienen un esquema de secciones que procura ajustarse a la secuencia de pasos del método de investigación asumido para el respectivo capítulo. 
- El esquema de secciones de los capítulos 5, 6 y 7 se conforman, entre otras que son propias de la presentación de los resultados, por las secciones de introducción, revisión de la literatura, discusión y conclusiones. En la sección de introducción se relacionan las principales nociones que trata el capítulo para dar paso, en la sección de revisión de literatura, a destacar las aportaciones de los estudios más recientes respecto a tópicos puntuales en los que se focaliza el análisis. En la sección de discusión se genera el debate en torno a la interpretación e implicaciones de los resultados obtenidos y las posibles limitaciones del trabajo. En la sección de conclusiones se presenta el valor agregado y las líneas de trabajo futuro.

- En el capítulo 8 se introduce una sección en la que se detalla la construcción progresiva de la carrera investigadora de la doctoranda durante el desarrollo de la tesis.

- Las fórmulas matemáticas, tablas y figuras se insertan y referencian siguiendo una secuencia numérica supeditada al número del capítulo. Mientras que las notas a pie de página siguen una secuencia ordinal en toda la extensión del documento.

- Las referencias bibliográficas y los anexos se presentan al final del documento. 


\section{CAPÍtULLO 2}




\title{
Capítulo 2
}

\section{Contextualización del problema de estudio: ZODES Magdalena Medio (Bolívar, Colombia)}

\begin{abstract}
Resumen: en este capítulo se describe la situación real en la que se origina el problema de transporte y que requiere el diseño estratégico-táctico de la red de transporte intermodal. La situación toma como referente las condiciones de acceso y conectividad de la Zona de Desarrollo Económico y Social (ZODES) Magdalena Medio del departamento de Bolívar en Colombia, y el potencial de la industria agroalimentaria para esta subregión. Las ZODES son zonas geoestratégicas de tipo espacial que se distinguen entre sí por aspectos geográficos, socioeconómicos y culturales. Desde estas dimensiones, la interpretación y comprensión del problema de transporte en la ZODES Magdalena Medio permite definir un marco de análisis para desarrollar los modelos de programación matemática que se desarrollan en los siguientes capítulos.
\end{abstract}




\subsection{Introducción}

El desarrollo de una investigación requiere de una serie de actividades que dependen sustancialmente del problema de investigación. Esencialmente, hay dos pasos involucrados para definir este problema: comprender a fondo la situación real y describirla desde un punto de vista significativo y analítico para abordar el problema (Kumar, 2008). A partir de estos dos pasos, se presenta en este capítulo una descripción de la situación real que origina el problema de transporte que se analiza en esta tesis. En la sección 2.2. se contextualiza la zona de estudio desde su relevancia para el transporte. En la sección 2.3. se definen las principales limitaciones que impiden el desarrollo de la actividad de transporte en la zona de estudio y su incidencia en las condiciones sociales y medioambientales. En la sección 2.4. se sustenta la idoneidad y pertinencia de analizar el problema definido.

\subsection{ZODES Magdalena Medio}

En esta sección se sintetizan, desde la perspectiva del transporte, los rasgos más distintivos de la Zona de Desarrollo Económico y Social (ZODES) Magdalena Medio. En la subsección 2.2.1. se realiza una caracterización física de la ZODES, en la subsección 2.2.2. se precisa la estructura productiva, y en la subsección 2.2.3. se describe la cadena de suministro agroalimentaria como fuente generadora de los flujos de transporte.

\subsubsection{Geografía y territorio}

La ZODES Magdalena Medio es una de las 7 subregiones que conforman el departamento de Bolívar, este a su vez es uno de los 32 departamentos que integran el territorio nacional de Colombia $^{6}$. En la Figura 2.1.a. se muestra la ubicación del departamento de Bolívar. Este departamento tiene una extensión aproximada de $25.978 \mathrm{Km}^{2}$, cuya ubicación y forma alargada facilita la conexión con el resto del país y el comercio exterior a través del mar Caribe (MazaÁvila et al., 2015). Esta ubicación le ha permitido convertirse en un epicentro importante de procesos de desarrollo económico, explotación de recursos naturales y eventos culturales y políticos de influencia regional y nacional (Gobernación de Bolívar, 2016). No obstante, la orografía de la zona y los hechos históricos marcados por la violencia contribuyeron a la pérdida de sinergia al interior del departamento, a la vez que impulsaron la configuración orgánica de regiones económicas y sociales de algunos municipios con otros departamentos del país (Gobernación de Bolívar, 2020a; Pita-Pico, 2016).

Tales aspectos propiciaron la identificación de retos estratégicos y apuestas productivas para compensar los desequilibrios generados en el departamento y promover de manera articulada su progreso, por lo tanto, se conformaron siete subregiones denominadas ZODES. Estas se muestran en la Figura 2.1.b. Las $\mathrm{ZODES}^{7}$ son zonas geoestratégicas de tipo espacial que se componen por un limitado número de municipios que comparten características geográficas, socioeconómicas y culturales. La que nos ocupa en esta tesis, la ZODES

\footnotetext{
${ }^{6}$ Colombia está organizada, con fines políticos y administrativos, en 32 departamentos, 1123 municipios y 11 distritos; los departamentos funcionan como entes de coordinación entre la nación y los municipios. El departamento de Bolívar está constituido por 44 municipios y 2 distritos (DANE, 2021).

${ }^{7}$ Con las ordenanzas departamental No. 12 de 2001 y No. 188 de 2017 se propone la conformación de las siete (7) ZODES, estas son: Norte, Dique, Montes de María, Isla de Mompox, Mojana, Loba y Magdalena Medio.
} 
Magdalena Medio, se sitúa al sur del departamento y está representada por los municipios de Arenal, Cantagallo, Morales, San Pablo, Santa Rosa del Sur y Simití. Los mencionados municipios constituyen en conjunto algo más del 34.5\% del territorio del departamento de Bolívar (Amézquita-López et al., 2018). La ZODES tiene una población proyectada para el año 2021 de 124.052 habitantes, equivalente al 5.61\% de la población departamental (Departamento Administrativo Nacional de Estadística [DANE], 2021); y ocupa la tercera posición entre las ZODES con la menor densidad poblacional, a razón de los desplazamientos forzados producidos por los hechos de conflicto armado y violencia que se presentan en el país desde hace más de 60 años (Comisión de la Verdad, 2020).

\section{Figura 2.1. Ubicación del departamento de Bolívar y la ZODES Magdalena Medio}

a.

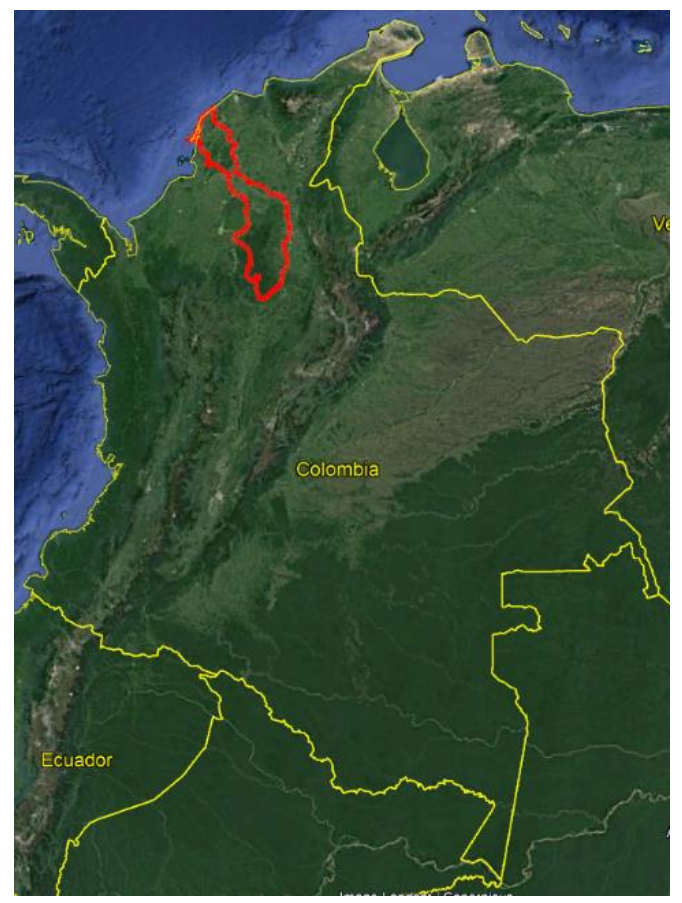

b.

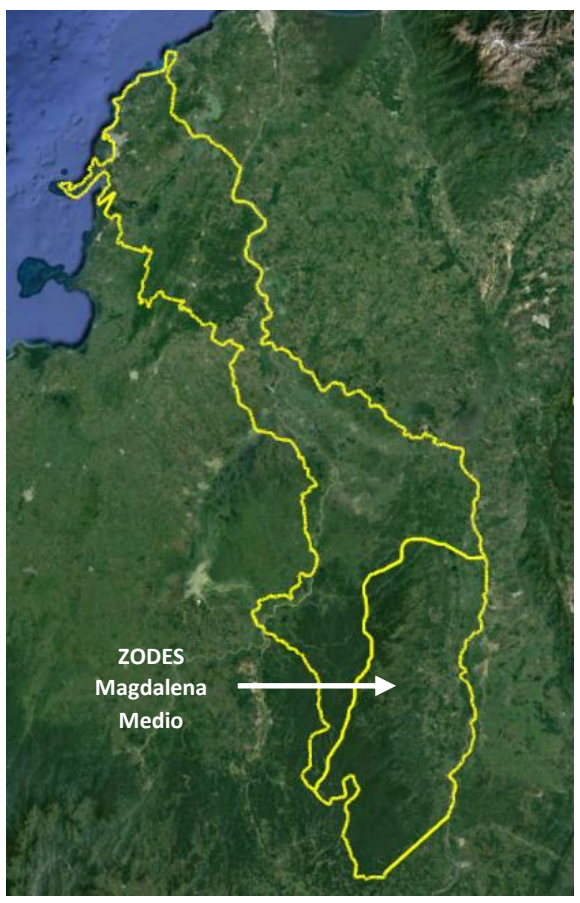

Fuente: Adaptado de Google Earth

El departamento de Bolívar se distingue por una fisiografía en la que destacan: bahías, áreas cenagosas e inundables generadas por afluentes, caños y ciénagas que forman los Ríos Magdalena, San Jorge y Cauca, así como de un relieve variado que alcanza hasta los 2.300 metros sobre el nivel del mar (Gobernación de Bolívar, 2020b). En lo que respecta a la ZODES Magdalena Medio, el territorio tiene una relieve caracterizado, al occidente como se muestra en la Figura 2.2.a., por las estribaciones de la cordillera central conocida como Serranía de San Lucas que le permite alcanzar alturas hasta los 2.000 metros sobre el nivel del mar y, al oriente, por el Río Magdalena. La Serranía de San Lucas es uno de los enclaves selváticos más importantes del país debido a su atractivo biológico para la conservación de flora y fauna (Silva, 2012). La biodiversidad registrada en sitúa 1.093 especies de plantas, 587 de aves, 191 de mamíferos, 185 de mariposas, 129 de peces y otros números significativos de especies de escarabajos coprófagos, anfibios, reptiles, y macroinvertebrados acuáticos (Parques Nacionales Naturales de Colombia, 2021). 
El Río Magdalena es el principal eje fluvial de Colombia. Como se puede ver en la Figura 2.2.b este Río atraviesa casi la totalidad del país de sur a norte con una extensión de $1.550 \mathrm{~km}$ y conecta a 129 municipios ribereños con dos de los puertos marítimos más importantes del país: el puerto de Cartagena (ubicado en el departamento de Bolívar) y el puerto de Barranquilla. El Río se divide en tres cuencas: alta, media y baja. Los municipios que conforman la ZODES Magdalena Medio confluyen en la cuenca media (ver Figura 2.2.c.). Esta tiene un recorrido de $1.100 \mathrm{~km}$ de extensión, es el tramo con mayor actividad de navegación, y conecta con los puertos marítimos de Cartagena y Barranquilla por el canal del dique y la desembocadura del Río en Bocas de Ceniza, respectivamente (ver Figura 2.2.b.). Entre los años 2013 a 2018 el flujo de carga por el Río se incrementó en $132 \%$ y en el año 2020 el tráfico portuario representó el 1,2\% del tráfico portuario nacional (Corporación Autónoma Regional del Río Grande de la Magdalena [CORMAGDALENA], 2021), por lo que se constituye como un importante eje para la integración de los municipios ribereños, así como del desarrollo e integración de sus áreas de influencia (Departamento Nacional de Planeación [DNP], 2021).

\section{Figura 2.2. Características geográficas del territorio}

a.

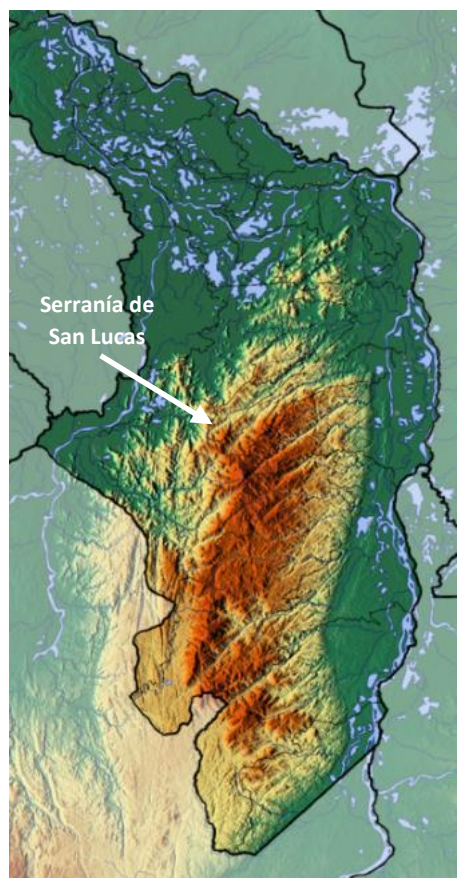

b.

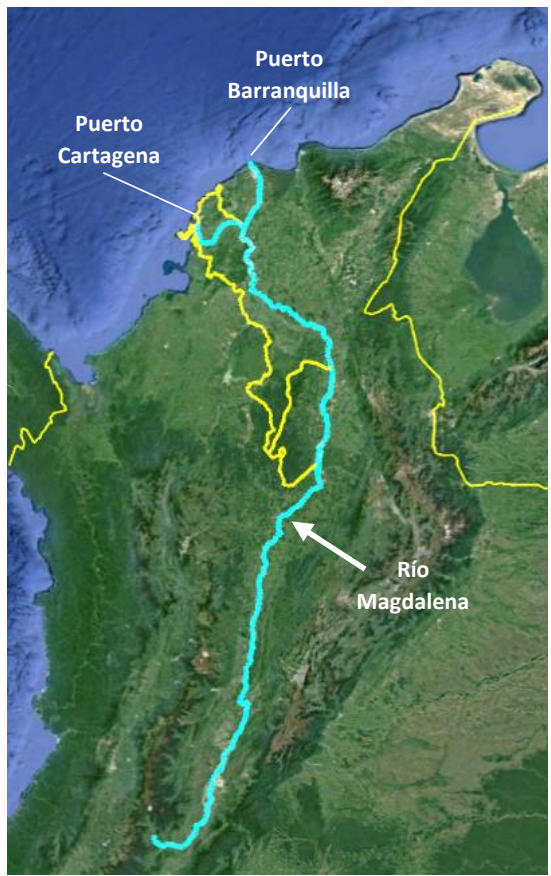

Fuente: Adaptado de Google Earth c.

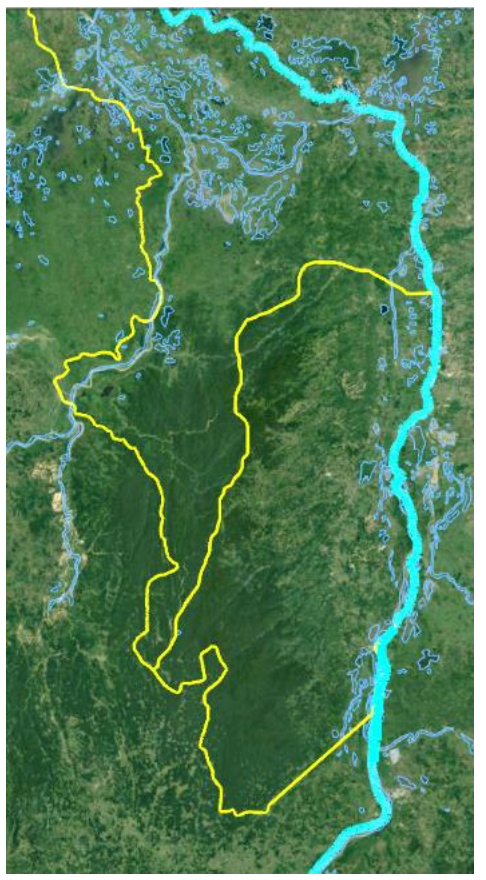

\subsubsection{Estructura productiva}

La ZODES Magdalena Medio, aunque es rica en dotación de recursos naturales y biodiversidad, tiene un gran potencial agroexportador que convierte a la industria agrícola en una de sus principales actividades económicas y productivas (Gobernación de Bolívar, 2020b). Con solo el 9\% del suelo apto para la agricultura, del cual apenas se aprovechan tres quintas partes, esta ZODES representa para el departamento de Bolívar el $21 \%$ del área cultivada y el 
$15 \%$ del producto agrícola comercializable. De las 15 alternativas de producción ${ }^{8}$, entre cultivos transitorios y permanentes ${ }^{9}$, los cultivos de palma de aceite, yuca, plátano y maíz agrupan el 92\% del potencial de comercio agrícola de la ZODES (Red de Información y Comunicación del Sector Agropecuario de Colombia [AGRONET], 2021). La Figura 2.3. muestra la expansión del área total sembrada por municipio, entre los años 2010 y 2018. En general se destaca un crecimiento significativo de la actividad agrícola en el municipio de San Pablo. Por su parte, en la Figura 2.4. se representa la participación de cada municipio en el producto total cosechado y el porcentaje de variación de la producción respecto al año anterior $^{10}$. Pese a que en los años 2011 y 2015 se reducen las toneladas de producto cosechado, la producción de la ZODES ha crecido alrededor del $46 \%$ en el periodo de análisis ${ }^{11}$.

Figura 2.3. Área sembrada por municipio de la ZODES Magdalena Medio, años 2010 a 2018

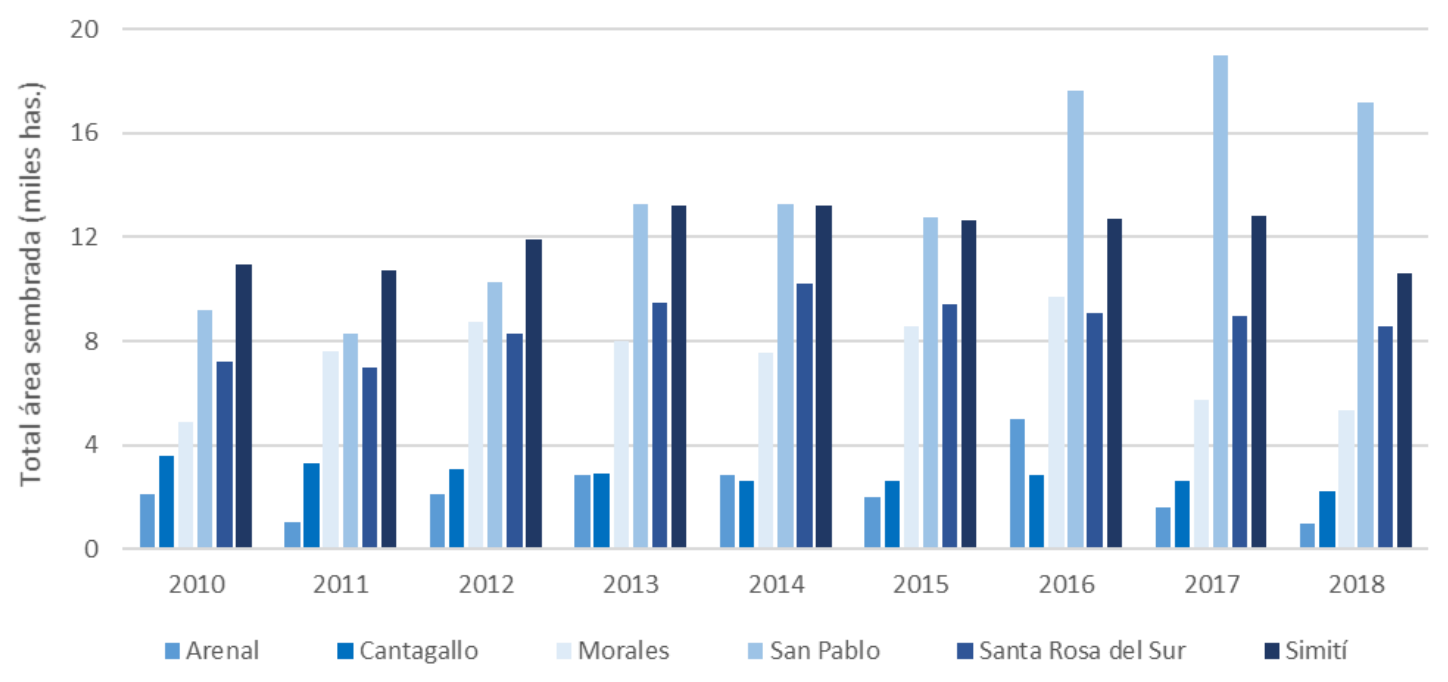

Fuente: Elaboración propia a partir de AGRONET (2021)

\footnotetext{
${ }^{8}$ Los productos agrícolas cultivados en el año 2018 son: algodón, arroz, cacao, café, caña panelera, caucho, frijol, maíz, palma de aceite, patilla, pitahaya, plátano, yuca (AGRONET, 2021).

${ }^{9}$ Los cultivos transitorios se caracterizan por tener un ciclo de crecimiento menor a un año, que después de la cosecha deben sembrarse nuevamente. Los cultivos permanentes o perennes son los que después de sembrados requieren de un tiempo relativamente largo de edad productiva para cosechar de manera continuada (según el ciclo del producto) y que no se deben volver a sembrar (DANE, 2021).

${ }^{10}$ En el anexo A se relacionan los datos de área sembrada y producción por alternativa agrícola, municipio y año.

${ }^{11}$ La variación negativa de la producción se puede sustentar por distintas causas. Entre estas, las dificultades de acceso en algunas áreas de la ZODES y la presencia de grupos ilegales que impiden censar toda el área sembrada, la pérdida del producto en la etapa de crecimiento ocasionada por inundaciones, la pérdida de producto en origen debido al reducido margen de beneficio en la comercialización. Ante la última causa también es posible que no se coseche el producto.
} 
Figura 2.4. Producción por municipio de la ZODES Magdalena Medio, años 2010 a 2018

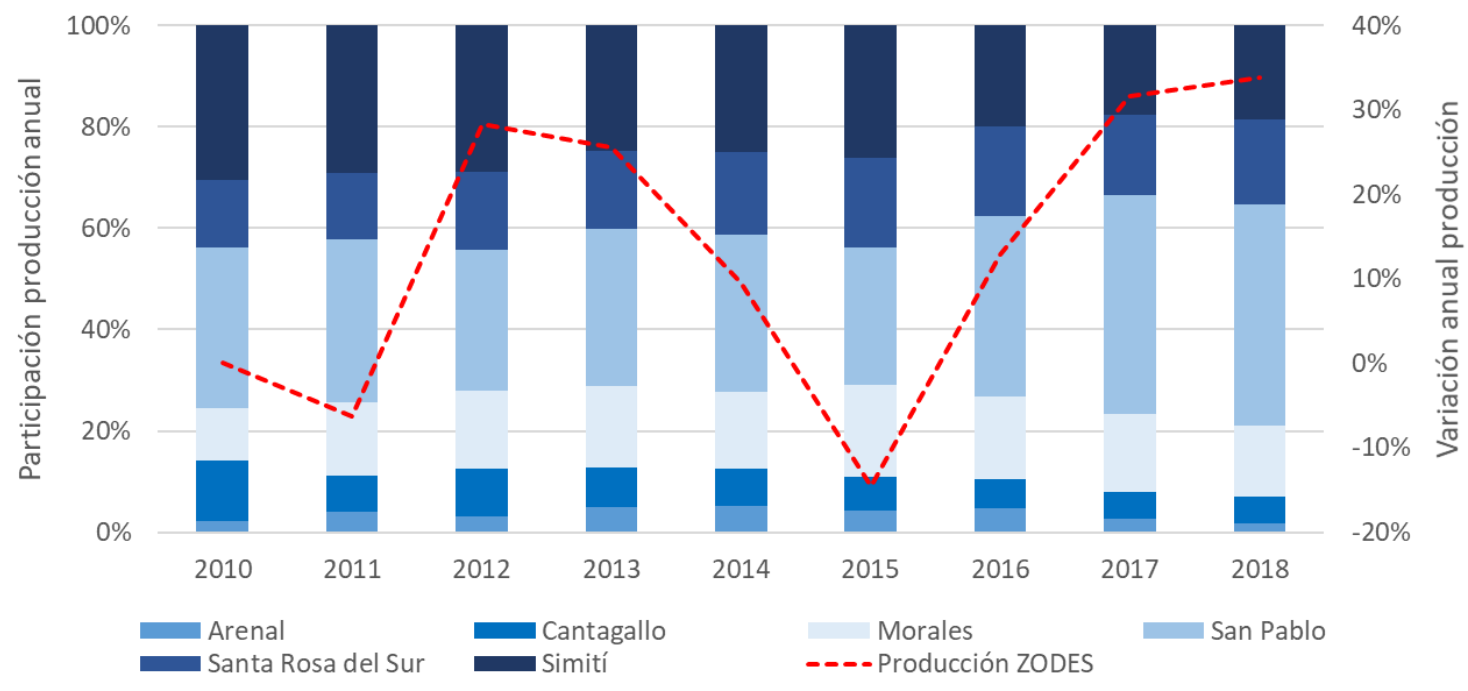

Fuente: Elaboración propia a partir de AGRONET (2021)

\subsubsection{Cadena de suministro agroalimentaria ${ }^{12}$}

La diversidad y temporalidad de los cultivos que conforman la estructura productiva agrícola de la ZODES conllevan a una identificación heterogénea de canales de distribución y actores que participan en la cadena de suministro. En otras palabras, el número de eslabones de la cadena pueden ser distintos en las diferentes alternativas de productos agrícolas. Para efectos de precisar los principales agentes económicos se unifica la estructura de la cadena con los siguientes actores: agricultores, puntos de acopio, empresas de transformación, distribuidores (mayoristas y minoristas, nacional e internacional), intermediarios y los clientes finales (nacional e internacional). En la Figura 2.5. se muestra una representación genérica de la cadena de suministro agroalimentaria de la ZODES Magdalena Medio.

Los agricultores están en el primer eslabón de la cadena. Estos se encargan de la limpieza y adecuación del terreno para la siembra y cosecha del producto, algunos desarrollan sus actividades de manera tecnificada y otros de modo manual. El área de siembra difiere entre los agricultores según el número de hectáreas que posean, por tanto, en algunas partes puede existir mayor concentración o dispersión de agricultores. Los puntos de acopio son lugares donde se dispone el producto para su agrupación, limpieza y/o empaque. Estos puntos, dependiendo del producto, pueden estar en las proximidades de la frontera agrícola o en los centros urbanos más cercanos, y facilitan la comercialización del producto y participación en el mercado. Las empresas de transformación se encargan de la elaboración de subproductos a partir del procesamiento del producto agrícola. Los distribuidores, mayoristas y minoristas, nacionales o internacionales, pueden ser uno o varios eslabones en la cadena y definen los canales de comercialización hasta el cliente final. Para algunos productos se identifica la participación de intermediarios. Estos suelen comprar grandes cantidades de producto o subproductos para

${ }^{12}$ Ésta subsección se construye a partir de las entrevistas y conversaciones que se llevaron a cabo, entre los meses de septiembre de 2016 y marzo de 2017, por la autora de esta tesis junto a docentes-investigadores y el equipo de apoyo vinculados al Instituto de Políticas Públicas Regional y de Gobierno (IPREG) de la Universidad de Cartagena (Colombia). 
venderlo a otros actores, a nivel nacional o internacional, por lo que pueden encontrarse aguas arriba y/o aguas debajo de la cadena.

Figura 2.5. Representación genérica de la cadena de suministro agroalimentaria de la ZODES Magdalena Medio

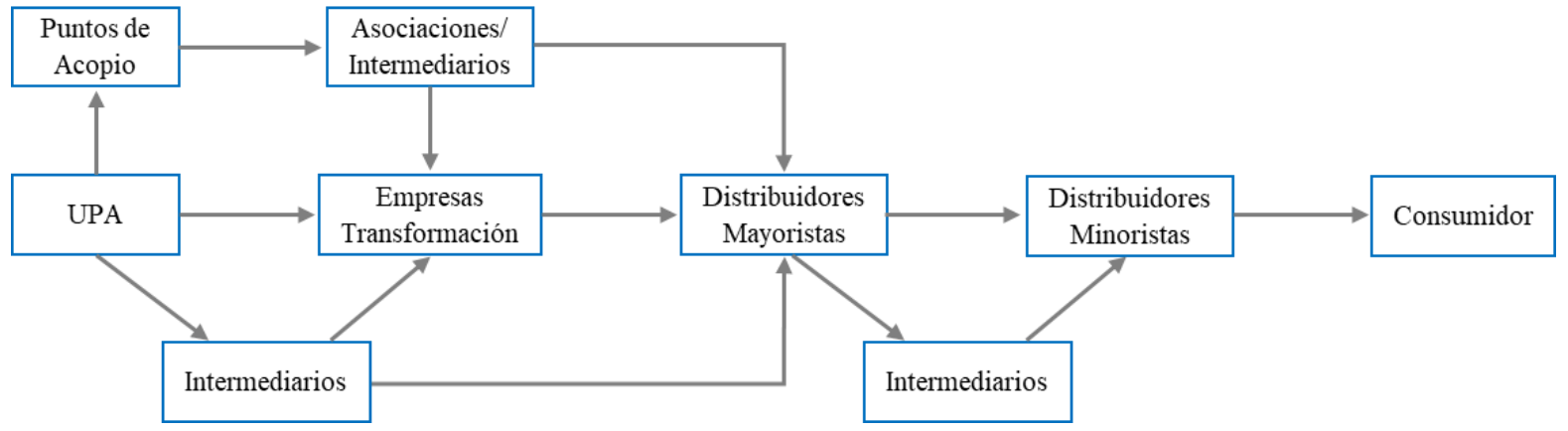

Fuente: Elaboración propia

Para esta cadena de suministro también influyen los intereses de ciertos grupos que apoyan, controlan o regulan el adecuado planeamiento y desarrollo de las actividades de los distintos eslabones identificados. Entre estos se encuentran las asociaciones de agricultores, entidades gubernamentales y la población en general. Las asociaciones son agrupaciones de agricultores que operan en pro de la competitividad del producto y el sector, a partir del apoyo en los procesos de cultivo y siembra, transferencia de tecnología y comercialización de los productos. De los entes gubernamentales se pueden distinguir: los que garantizan el cumplimiento de estándares sanitarios y fitosanitarios para la comercialización de los productos, los que participan y lideran procesos de investigación e innovación que impulsa el desarrollo científico del sector y la transferencia tecnológica, y los que formulan políticas públicas y diseñan las estrategias de inversión para promover el desarrollo competitivo, equitativo y sostenible del sector. Finalmente, la población, que está representada por los habitantes de las zonas de influencia de cada uno de los municipios que conforman la ZODES Magdalena Medio.

\subsection{Descripción del problema}

En esta sección se definen los principales aspectos que desencadenan el problema de transporte en la ZODES Magdalena Medio y las repercusiones sociales y medioambientales en su área de influencia. En la subsección 2.3.1. se exponen las implicaciones del transporte en la comercialización del producto agrícola, en la subsección 2.3.2. se desglosa la composición modal de las redes de transporte con influencia en la ZODES, y en la subsección 2.3.3. se presentan consideraciones sociales y medioambientales relevantes que pueden influir en la solución al problema. 


\subsubsection{Comercialización y gestión del transporte ${ }^{13}$}

La Tabla 2.1. resume datos de la cadena agroalimentaria de la ZODES. Pese a la importante representatividad de la agricultura de esta subregión para el departamento de Bolívar, no hay un tejido empresarial robusto y con suficiente tecnología para generar valor añadido a la cadena. Las áreas destinadas a la actividad agrícola están organizadas como unidades de producción agropecuaria (UPA) ${ }^{14} \mathrm{y}$, en algunos casos, los agricultores se agrupan en asociaciones para comercializar el producto a precios competitivos, no todos tienen el suficiente poder de negociación para establecer lazos comerciales con las empresas de transformación y/o los distribuidores mayoristas que se ubican en otras zonas del país y el extranjero. Por lo tanto, la participación de los intermediarios es recurrente en el proceso de comercialización. El número, capacidad y la carencia de condiciones adecuadas para la manipulación del producto en los puntos de acopio no permite la consolidación y almacenamiento de grandes cantidades de producto, además que, de estos solo se benefician los actores de la cadena productiva en la que participa el punto de acopio. Las empresas de transformación ubicadas en la ZODES y en el territorio nacional generan valor agregado, respectivamente, para 2 y 3 de las 15 cadenas productivas identificadas. Los demás productos se comercializan como commodities.

Tabla 2.1. Datos de la cadena de suministro agroalimentaria de la ZODES Magdalena Medio

\begin{tabular}{ll}
\hline Datos & \\
\hline 190 & Mil toneladas de producto cosechado \\
5996 & UPAs \\
3 & Puntos de acopio \\
4 & Microempresas de transformación \\
17 & Intermediarios \\
+20 & Distribuidores \\
\hline
\end{tabular}

Fuente: IPREG, DANE (2021)

La reducida posibilidad de consolidar cantidades significativas de producto en los puntos de acopio repercute en las actividades de agrupación y empaque, a su vez que condiciona la gestión del transporte. En el primer eslabón de la cadena se identifican al menos dos estrategias para efectuar el transporte, esta gestión implica la negociación del flete para cumplir con el pedido solicitado. En la primera estrategia, los agricultores gestionan de manera individual el transporte desde la UPA hasta el punto de acopio y desde este, a través de las asociaciones o intermediarios, hasta la empresa de transformación o el distribuidor. Los agricultores asumen los costes de transporte en su totalidad. En la segunda estrategia, son los intermediarios y/o empresas de transformación los que gestionan el transporte desde las UPAs hasta la empresa

\footnotetext{
${ }^{13}$ Ibíd 12.

${ }^{14}$ Para que éstas áreas se consideren una UPA deben cumplir con las siguientes condiciones: produce bienes agrícolas, forestales, pecuarios y/o acuícolas; tiene al menos un único productor/a natural o jurídico que asume la responsabilidad y los riesgos; y, utiliza al menos un medio de producción como construcciones, maquinaria, equipo y/o mano de obra (DANE, 2021).
} 
de transformación o el distribuidor mayorista. Estos se encargan de agrupar el producto siguiendo una ruta definida previamente con los agricultores, y asumen la totalidad del flete. Es la estrategia más utilizada y aunque pareciera ventajosa, supone para los agricultores reducir los precios, limitando a mínimos el margen de beneficio. En las demás etapas de la cadena la gestión del transporte no dista sustancialmente de las estrategias definidas en el mercado.

El flete varía, inclusive, para una misma estrategia adoptada. Las principales razones están dadas por la insuficiente disponibilidad de redes de transporte y el estado físico de las existentes, así como por el modo de transporte, la reducida capacidad del vehículo modal y la limitada disponibilidad de operadores de transporte. Bajo estas condiciones se dificulta la estandarización de las tarifas y, en general, se incurre en altos costes de transporte. Los altos costes se producen, sobre todo, para mover el producto desde las UPAs. Por otra parte, la característica perecedera de los productos agrícolas supone pérdidas, tanto en cantidad como en calidad, en todos los procesos de la cadena (Nourbakhsh et al., 2016). En la ZODES las condiciones de acopio y transporte del producto conducen a que estas pérdidas físicas y sus respectivos costes sean soportadas por las UPAs. Se estima que las pérdidas físicas de producto pueden llegar a representar hasta el $15 \%$ del total cosechado ${ }^{15}$. Los costes por pérdidas varían según el producto, el área de cultivo y la época del año.

\subsubsection{Redes de transporte}

El principal modo de transporte empleado en el departamento de Bolívar es el transporte por carretera, sin embargo, las redes presentan un déficit, no cubren adecuada y equilibradamente el territorio, e impiden la integración física del mismo (Gobernación de Bolívar, 2020a). Por su parte, el transporte por modo fluvial a través del Río Magdalena representa una gran ventaja competitiva, no obstante, ha tenido inconvenientes de acceso y costes elevados debido a la inviabilidad de navegar en algunas secciones del Río a causa de la erosión, deforestación, desecación de ciénagas y la contaminación (CORMAGDALENA, 2021). En esta subsección se describe el estado de las redes de transporte por carretera, fluvial y las potenciales conexiones ferroviarias en la ZODES Magdalena Medio.

\subsubsection{Carretera}

La red de carreteras del departamento de Bolívar tiene una extensión de $3.099 \mathrm{~km}$, de la cual el $15 \%$ son vías primarias, el $4 \%$ secundarias y $81 \%$ terciarias ${ }^{16}$. Poco más de la mitad de la red primaria y secundaria se encuentra en buen estado, mientras que, la red terciaria, en su

\footnotetext{
${ }^{15}$ Los reducidos márgenes de beneficios en la comercialización del producto y las dificultades de acceso a la subregión sustentan la ocurrencia en las pérdidas de producto en la ZODES. Estas no permiten tener un registro total de la actividad agrícola en la ZODES, por lo que es posible que este porcentaje sea aún mayor.

${ }^{16}$ El sistema nacional de carreteras en Colombia clasifica las carreteras según su funcionalidad en: primarias, secundarias y terciarias. La red primaria está constituida por las carreteras que unen las principales ciudades entre sí, y con los puertos marítimos, fronteras terrestres y otros nodos de intercambio modal. Esta red es básica para la integración y competitividad del país, pues une a las áreas de producción con las de consumo y debe estar pavimentada. La red secundaria está compuesta por las carreteras que unen las cabeceras municipales entre sí y/o que provienen de una cabecera municipal y conectan con una carretera primaria. Estas pueden funcionar pavimentadas o en afirmado. La red terciaria cumple una función de interconexión entre las veredas, las cabeceras municipales y las carreteras departamentales; por lo menos deben funcionar en afirmado (Instituto Nacional de Vías [INVIAS], 2021a; Ospina, 2016).
} 
mayoría, tiene un estado regular o malo (Gobernación de Bolívar, 2020a; Maza-Ávila et al., 2015). En la Figura 2.6.a. se muestra la red de carreteras primaria y secundaria del departamento de Bolívar, en la 2.6.b. la red terciaria y en la 2.6.c. los tramos del proyecto vial Transversal de las Américas con incidencia en la ZODES que se encuentra en la fase de ejecución. Las tres figuras permiten ver el desequilibrio en la cobertura vial entre la parte norte y sur del departamento, y en lo que respecta a la ZODES se manifiesta el déficit de este tipo de infraestructura y la desconexión entre los municipios. Estas condiciones dificultan la conectividad y accesibilidad, e influye en los flujos de transporte. Dado que las vías se encuentran en mal estado, se ve restringida la utilización de un parque automotor con grandes capacidades para el transporte de la carga. Los requerimientos de transporte del producto conllevan al uso de distintos vehículos modales con distintas capacidades. El producto se transporta a granel y en unidades de empaque que no superan los $50 \mathrm{kgs}$.

\section{Figura 2.6. Red de carreteras del departamento de Bolívar y proyectos vigentes en la ZODES Magdalena Medio}
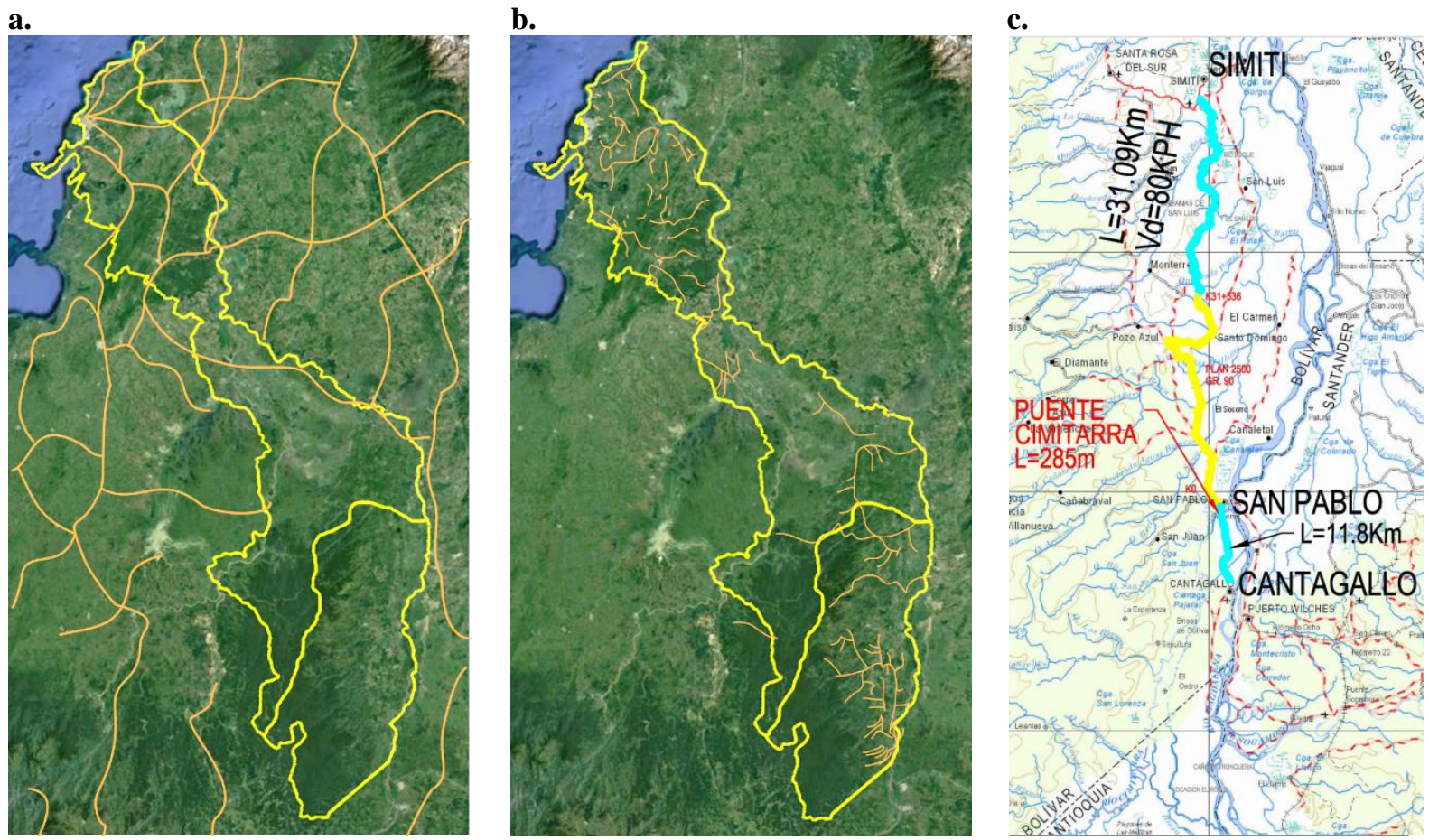

Fuente: Elaboración propia a partir de Agencia Nacional de Infraestructuras [ANI] (2021), INVIAS (2021b) y Sociedad Vías de las Américas (2021)

\subsubsection{Fluvial}

El transporte fluvial a través del Río Magdalena ha sido lento y segmentado, careciendo de integración, comunicación e incompatibilidad con los otros modos de transporte. Si bien el Río se considera un pilar estratégico en la formulación de lineamientos para impulsar el desarrollo del país, la navegabilidad ha sido deficiente debido al requerimiento de obras de dragado, mantenimiento y monitoreo de los canales navegables ${ }^{17}$, tanto como por la escasa

${ }^{17}$ La red fluvial de Colombia divide los canales navegables en corredores fluviales de carga y corredores de pasajeros y mercancías. Los primeros cuentan con condiciones de navegabilidad para vehículos con capacidad superior a las 25 toneladas, 
infraestructura de muelles y puertos (Corredor \& Díaz, 2018). La Figura 2.7.a. muestra las secciones navegables del Río y los puertos fluviales ubicados en su ribera oriental, por medio de los cuales los agricultores de la ZODES realizan actividades de consolidación de producto. Por su parte, en la subregión hay poca disponibilidad de infraestructuras de transferencia que permiten gestionar grandes volúmenes de carga. El quehacer tradicional en la movilidad de mercancías y pasajeros ha permitido identificar algunos puntos como referentes potenciales para la inversión de instalaciones intermodales (Amézquita-López et al., 2018).

La Figura 2.7.b. muestra los corredores navegables al interior de la ZODES. En estos, el producto agrícola se mueve en pequeñas unidades de empaque que oscilan entre los 20 y 50 kgs., se emplea principalmente el ferry como vehículo modal, y tienen un reducido equipamiento para realizar las actividades de consolidación y transferencia de la unidad de carga. Los flujos de transporte, desde la ZODES hacia puertos marítimos o zonas del territorio nacional, se direccionan al norte para conectar con el puerto fluvial de Gamarra y al sur para conectar con el puerto fluvial de Puerto Wilches. Los dos puertos se ubican en la ribera oriental del Río y cuentan con equipamiento especializado. Estos corredores también se utilizan en el flujo de pasajeros, tanto que al sur de la ZODES predomina el transporte por este modo.

Figura 2.7. Red fluvial del departamento de Bolívar, puertos marítimos y fluviales, y corredores navegables en la ZODES Magdalena Medio

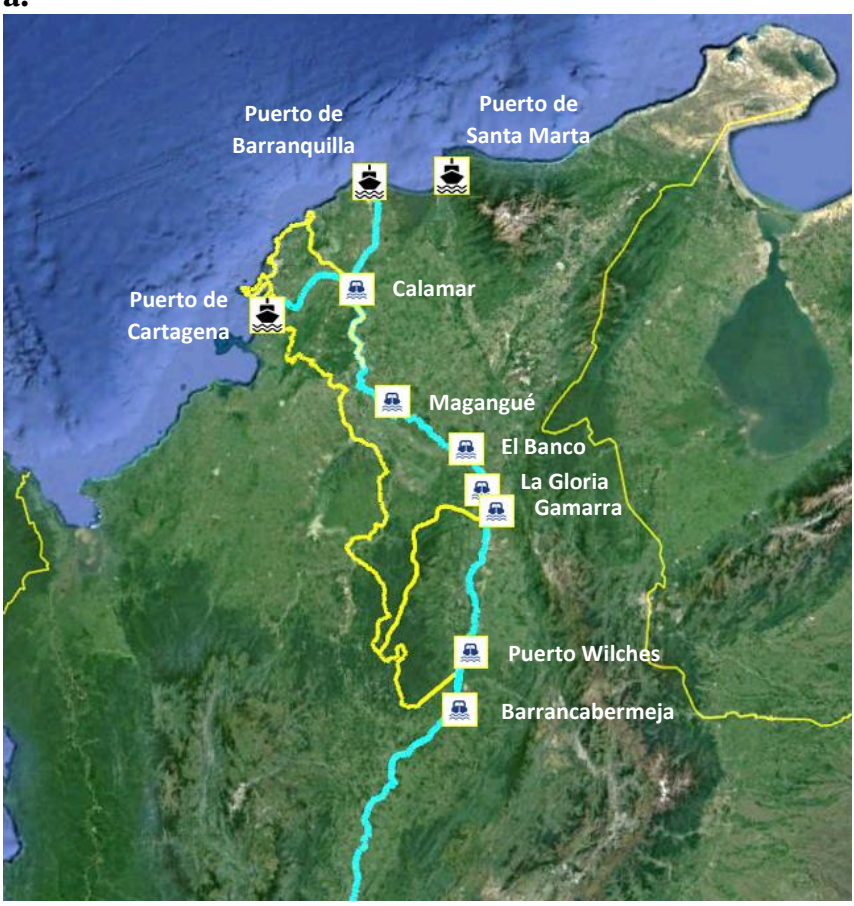

b.

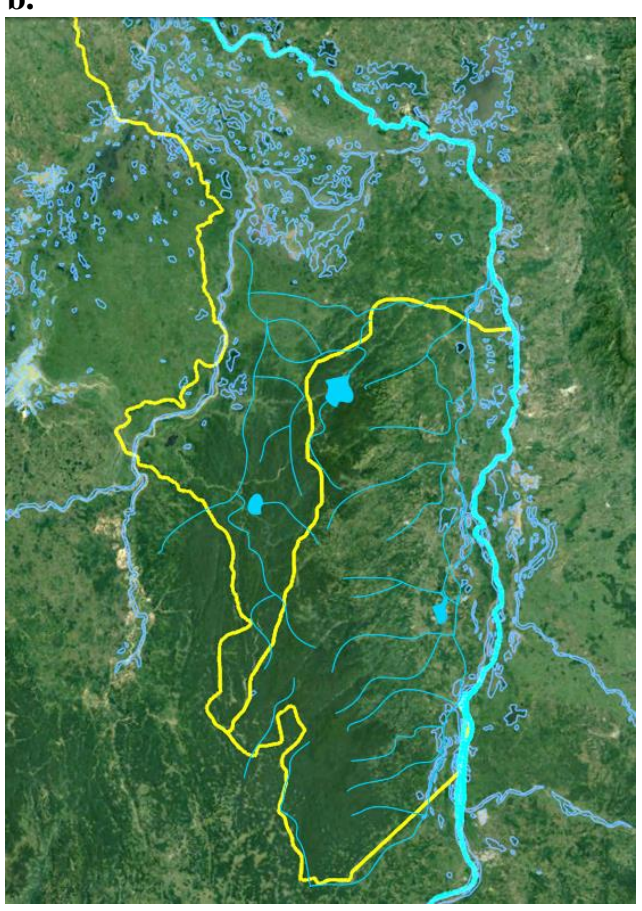

Fuente: Elaboración propia a partir de Gobernación de Bolívar (2020a) y ANI (2021)

los segundos, para vehículos con capacidad menor a 25 toneladas. Los corredores fluviales de carga pueden ser permanentes (navegables durante todo el año), o transitorios (navegables solo en temporada de lluvias) (DNP, 2021). 


\subsubsection{Conexión ferroviaria}

Colombia cuenta con $3.533 \mathrm{~km}$ de red férrea, de la cual el 49,1\% está inactiva, el 4,6\% es de uso privado y el porcentaje restante está concesionada; en los tramos activos el producto que más se transporta es el Carbón en cortas distancias (DNP, 2020). Las decisiones políticas nacionales han sido determinantes en el desarrollo de este modo de transporte, ya que se ha dado preferencia a proyectos que impulsan de manera contundente el transporte por carretera ocasionando un retraso en las iniciativas para fortalecer el transporte ferroviario (Márquez, 2017). Este retraso está sustentado por la inviabilidad técnica y económica que supone mantener y operar una red con un ancho de vía de $914 \mathrm{~mm}$ y por la débil red de instalaciones de interconexión modal existentes (Nieto, 2011). En la Figura 2.8. se detalla la red ferroviaria de Colombia. Aunque el departamento de Bolívar no tiene conexión directa con ninguna de las líneas férreas, los habituales movimientos de mercancías y pasajeros en la ZODES permiten identificar conexiones potenciales vía fluvial en la parte sur, y por carretera en la parte norte y centro.

Figura 2.8. Red ferroviaria de Colombia

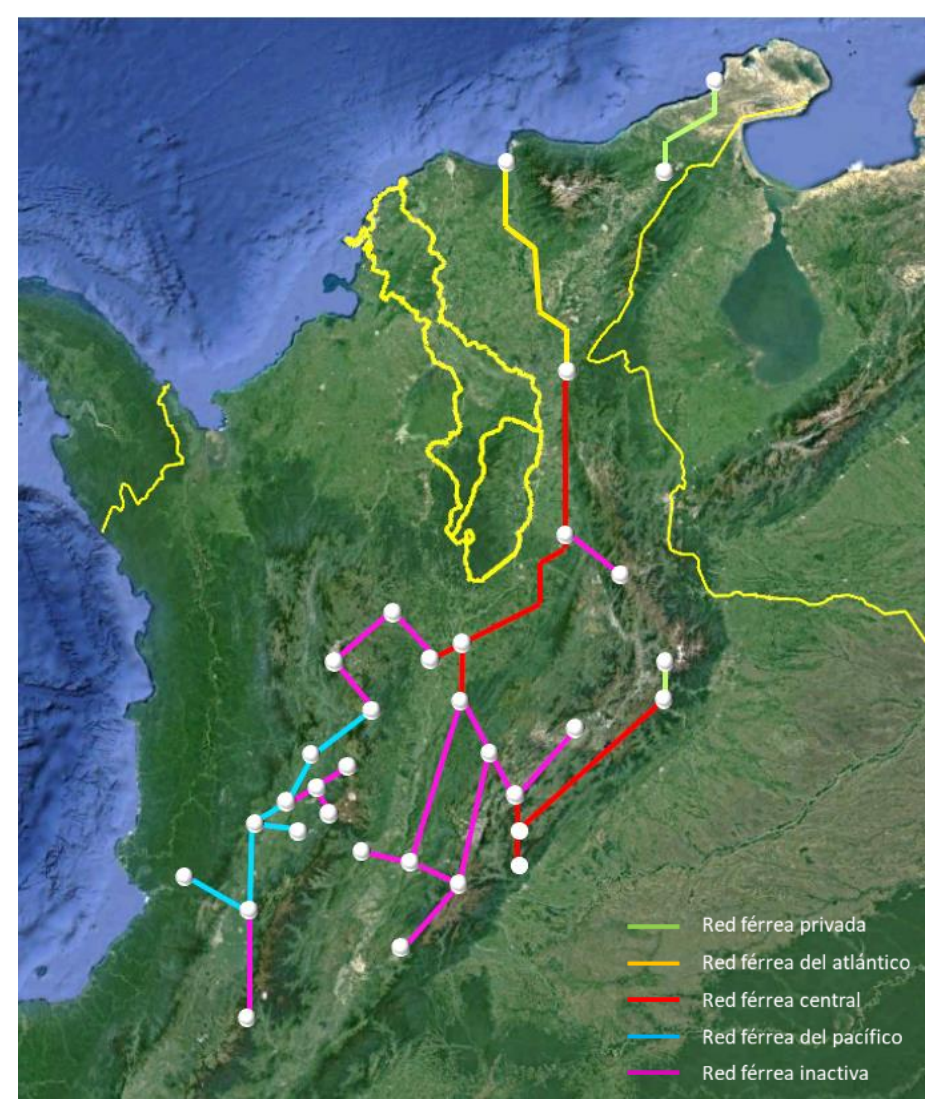

Fuente: Elaboración propia a partir de DNP (2020) y ANI (2021)

\subsubsection{Situación social y medioambiental}

Para relacionar la influencia de la situación social y medioambiental de la ZODES con las posibles alternativas de solución al problema parece pertinente reconocer situaciones puntuales como los movimientos migratorios, el conflicto armado, el desplazamiento y desaparición 
forzada, los cultivos ilícitos y la escasa presencia de la administración pública en el territorio por más de 50 años. Por ejemplo, el desplazamiento forzado de la población ocasionado por el conflicto armado no solo redujo la productividad agrícola del campo, también conllevó, entre otros, a la pérdida de la propiedad de la tierra por parte de los agricultores, el incremento de cultivos ilícitos y la apropiación de tierra para la ganadería extensiva por parte de terceros (Comisión de la Verdad, 2020). Sin embargo, un análisis profundo de cada una de estas situaciones dista del objetivo general de la tesis. En su caso se consideran las consecuencias generadas por estas situaciones, que permiten definir para la ZODES un panorama del estado actual de la sociedad.

El estado actual de la ZODES es muy sensible ya que acarrea, sobre todo, con altos índices de pobreza, analfabetismo, desempleo y falta de equipamiento social. De acuerdo con el índice de necesidades básicas insatisfechas (NBI) para el año 2018, en la ZODES la proporción de personas en NBI es de 39\%, mientras que, a nivel departamental y nacional la proporción es de $26.7 \%$ y $14.28 \%$, respectivamente (DANE, 2021). Por otra parte, el desempleo y las insuficientes oportunidades laborales y educativas suscitan la emigración, provocando una ruptura en el desarrollo generacional del campo y en la desintegración del núcleo familiar. Este último conduce, eventualmente, a que se resignifiquen los roles y la mujer tome una participación activa en las UPAs. Además, la apropiación de grandes extensiones de tierra por un reducido número de agricultores incentiva los desequilibrios en la cobertura del mercado y la distribución de las riquezas.

La apropiación de la tierra también pone en riesgo el uso adecuado de las fuentes de agua entre los agricultores, así como la pérdida progresiva de los activos medioambientales de la ZODES. A finales de los años 80s la cartografía reflejaba una gran reserva natural de bosques con escasos asentamientos en las cabeceras municipales de la ZODES. No obstante, el proceso de migración desde los departamentos vecinos, a comienzos de la siguiente década, propicio la conformación de nuevos asentamientos e impulsó la actividad agrícola y pecuaria (AmézquitaLópez et al., 2018). Esto a su vez condujo a la construcción espontánea de infraestructura de transporte sin los permisos requeridos, la expansión descontrolada de la frontera agrícola, la ganadería extensiva y la extracción ilícita de minerales con resultados que se enmarcan en la contaminación del Río Magdalena y demás corredores navegables, la transformación del paisaje y la deforestación de los bosques naturales (Alarcón et al., 2018; Marín et al., 2018).

Colombia, pese a tener un $51.6 \%$ del territorio ocupado por bosques naturales presenta altas tasas de deforestación, siendo la cuenca del Magdalena medio una de las más afectadas. Se estima que, de 1 millón de hectáreas de bosques identificadas en el año 1995 en la serranía de San Lucas, sobreviven menos del 12\% (Silva, 2012). La Figura 2.9. muestra los cambios en la cobertura de bosques naturales en el departamento de Bolívar, entre los años 1990 a 2016. En esta es posible destacar la pérdida de bosques estables y el incremento en la deforestación en el área de influencia de la ZODES Magdalena Medio, así como la reducción en las áreas de regeneración. La pérdida de bosques reestructura los ecosistemas y amenaza el equilibrio natural y la continuidad de las especies. Datos recientes permiten mencionar que, la zona sur del departamento Bolívar es el sexto núcleo del territorio colombiano con mayor pérdida de bosques naturales, solamente en el municipio de Santa Rosa del Sur se perdió el $29.4 \%$ de los bosques entre los años 2017 y 2018 (Flórez, 2018). Por ello no es adecuado alejar las alternativas de solución al problema de las realidades sociales y medioambientales de la ZODES. 
Figura 2.9. Cambios en la cobertura de bosques naturales en el departamento de Bolívar
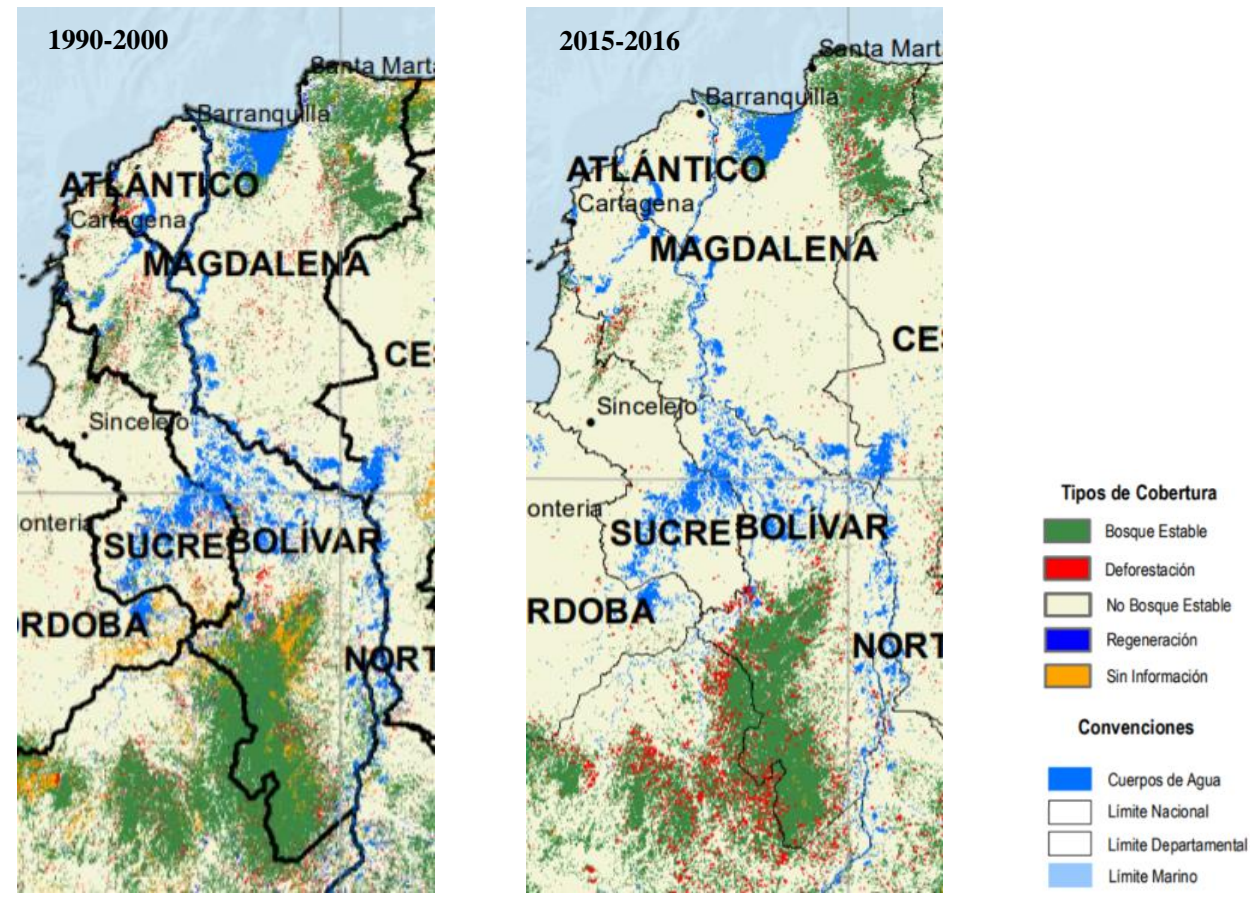

Fuente: Adaptado de Instituto de Hidrología, Meteorología y Estudios Ambientales (2021)

\section{4. ¿Por qué analizar este problema?}

Las problemáticas referidas a los sectores agropecuario y desarrollo rural, y de logística y transporte siempre han sido una asignatura pendiente para el país (DNP, 2021). En los últimos 20 años se han formulado acciones ambiciosas en los planes de desarrollo nacional que, en la etapa de ejecución, se ven menguadas y ajustadas debido a la limitada disponibilidad de recursos económicos, la inviabilidad técnica de algunos proyectos, las decisiones políticas y la corrupción en todos los niveles de gobierno. No obstante, la presión ejercida por el sector industrial, la visión de competitividad del país, la voluntad política, los Objetivos de Desarrollo Sostenible (ODS) emanados de la ONU y las estrategias de reactivación económica han permitido poner en marcha algunas acciones para recuperar y fortalecer estos dos sectores. En el Plan Nacional de Desarrollo 2018-2022 (DNP, 2019) se estableció el pacto por el transporte y la logística, a través del cual se propone potenciar la red fluvial y férrea de país, al tiempo que se mejora la eficiencia del transporte por carretera, aéreo y marítimo con el fin de reducir costes y tiempos; y el pacto por la sostenibilidad, que busca un equilibrio entre el desarrollo productivo y la conservación del medioambiente.

Bajo estas directrices, en la Política Nacional Logística, vigente desde el año 2020, se estableció como objetivo promover la eficiencia en el transporte a partir de: a) el transporte intermodal y la modernización de los servicios de transporte de carga, b) la facilitación del comercio optimizando la oferta de terminales, infraestructura y trámites de comercio exterior, y c) el desarrollo de mecanismos para fortalecer la institucionalidad, el uso de TIC y el capital humano (DNP, 2021). Con este planteamiento se pretende reducir a 9.5\% los costes logísticos para llegar a valores cercanos a los países de la OCDE, pues, a 2018, este coste representaba el $13.5 \%$ de las ventas; constituyendo el transporte poco más de la tercera parte del coste (Higuera, 2020). Sin embargo, en el departamento de Bolívar el porcentaje es más elevado, 
oscila entre $22 \%$ y $28 \%$, por lo que los desafíos para reducirlo son más rigurosos (Díaz, 2020). El déficit de infraestructura de transporte afecta, principalmente, el transporte de los bienes producidos desde el sector rural, pero también el acceso a otros bienes y los servicios básicos como educación, seguridad y salud (Gobernación de Bolívar, 2020a).

Bolívar debe afrontar grandes retos no solo en temas de infraestructura, también en lo referido a la educación, la sostenibilidad ambiental, el entorno para los negocios y el mercado laboral. La Tabla 2.2. muestra los resultados 2020-2021, de los 13 pilares que componen el Índice Departamental de Competitividad (IDC) ${ }^{18}$ y los correspondientes resultados de aquellos indicadores que se relacionan con la descripción presentada en la sección 2.3. En términos generales el departamento se ubica en una posición media-favorable, sin embargo, la desagregación del IDC y los indicadores marcan un panorama inquietante frente a las estrategias definidas en el Plan Regional de Competitividad de Cartagena y Bolívar 2008-2032. En dos de las apuestas productivas establecidas de este plan y que son de interés para esta tesis, la logística para el comercio exterior y la agroindustria, se trazaron estrategias focalizadas en la construcción de vías, la conectividad y la conexión intermodal.

Tabla 2.2. Resultados de los pilares e indicadores del IDC del departamento de Bolívar, año 2020

\begin{tabular}{|c|c|c|c|}
\hline Pilar & Posición (x de 33) & Indicadores & Posición (x de 33) \\
\hline Instituciones & 22 & Red vial primaria & 20 \\
\hline Infraestructura & 17 & Red vial a cargo del & \\
\hline Adopción TIC & 21 & departamento & 24 \\
\hline Sostenibilidad ambiental & 31 & Coste de transporte terrestre & 24 \\
\hline Salud & 12 & a mercado interno & 24 \\
\hline Educación básica y media & 17 & Tasas de deforestación & 31 \\
\hline $\begin{array}{l}\text { Educación superior y } \\
\text { formación para el trabajo }\end{array}$ & 12 & $\begin{array}{l}\text { Generación de emisiones de } \\
\mathrm{CO}^{2} \text { en fuentes fijas }\end{array}$ & 31 \\
\hline Entorno para los negocios & 18 & $\begin{array}{l}\text { Proporción de áreas } \\
\text { protegidas }\end{array}$ & 33 \\
\hline Mercado laboral & 20 & Formalidad laboral & 22 \\
\hline Sistema financiero & 20 & Brecha de participación & \\
\hline Tamaño del mercado & 4 & $\begin{array}{l}\text { laboral entre hombres y } \\
\text { mujeres }\end{array}$ & 27 \\
\hline $\begin{array}{l}\text { Sofisticación y } \\
\text { diversificación }\end{array}$ & 4 & Grado de apertura comercial & 4 \\
\hline $\begin{array}{l}\text { Innovación y dinámica } \\
\text { empresarial }\end{array}$ & 14 & $\begin{array}{l}\text { Inversión en calidad de la } \\
\text { educación básica y media }\end{array}$ & 32 \\
\hline IDC departamental & 11 & Cobertura en salud & 18 \\
\hline
\end{tabular}

Fuente: Adaptado de Consejo Privado de Competitividad de Colombia (2021)

\footnotetext{
${ }^{18}$ El Índice Departamental de Competitividad es un informe elaborado por el Consejo Privado de Competitividad de Colombia que tiene el objetivo de compilar las mediciones de diferentes aspectos que inciden en el nivel de competitividad de los departamentos del país y plantear recomendaciones que requieren coordinación pública y/o privada. El índice se obtiene a partir de la medición de 102 indicadores agrupados en 13 pilares y se define un ranking nacional considerando los 32 departamentos y Bogotá, la ciudad capital del país (Consejo Privado de Competitividad, 2021).
} 
Por tanto, es pertinente analizar las alternativas de solución al problema de la ZODES que guíen la toma de decisiones, a la luz de las iniciativas y acciones que impulsan la reactivación de los sistemas de transporte y sus interconexiones. Entre estos, la transformación del Río Magdalena como eje de integración e intermodalidad en Colombia, la consolidación de la operación ferroviaria y la inversión municipal y departamental en la construcción que favorezca la conectividad de los municipios de la ZODES. El Plan de Acción 2019-2021 trazado para el Río Magdalena se centra en la recuperación de la navegabilidad; sin embargo, las iniciativas definidas apoyan, pero no vinculan, el desarrollo portuario (CORMAGDALENA, 2021). En el Plan Maestro Ferroviario (DNP, 2020), a través de un esquema de inversiones prioritarias, se procura la complementariedad y articulación modal en la configuración carretero-ferroviario y fluvial-ferroviario. En el Plan de Desarrollo Departamental 2020-2023 (Gobernación de Bolívar, 2020a) se apuesta por la mejora de la infraestructura de transporte en pro de la productividad y competitividad del territorio y la calidad de vida de las personas, cuyas metas se centran en la intervención de las carreteras, la recuperación de corredores fluviales que conectan con el Río y el fortalecimiento de la agroindustria conformando organizaciones productivas y fomentando la tecnificación.

Para estudiar la situación descrita a lo largo de este capítulo y proponer alternativas de solución, en el capítulo siguiente se ajusta la dimensión del problema real en concordancia con la técnica de análisis de toma de decisiones que se utiliza en esta tesis: la programación matemática. El ajuste al problema se basa en identificar los requerimientos en la infraestructura de transporte conjugando la visión estratégica trazada por las entidades gubernamentales para este sector y los criterios de decisiones de los diferentes actores y grupos de interés de la ZODES. Desde esta visión estratégica se toma en cuenta que los requerimientos de transporte sean coherentes con soluciones de transporte intermodal, a partir de la consideración de aspectos económicos, sociales y medioambientales. 


$$
\text { CAPÍtulO } 3
$$




\section{Capítulo 3}

\section{Enfoque metodológico: programación matemática}

Resumen: en este capítulo se explica la sistemática llevada a cabo para transformar el problema del mundo real a un problema manejable con las técnicas de programación matemática. La aplicación de la secuencia de pasos conlleva, primero, a simplificar la situación real identificando el problema combinado de localización de instalaciones-diseño de red (LIDR), para luego, modelarlo y proponer las formulaciones que se desarrollan en los capítulos 5, 6 y 7. La modelación de los datos del problema y la estructura de la red de entrada favorecen la interpretación matemática del problema LI-DR, así como la implementación informática en dos esquemas de resolución cuyas estructuras de codificación se basan en los lenguajes $\mathrm{R}$ y Python. Con ambos esquemas de resolución se utiliza una licencia académica del software de optimización matemática Gurobi Optimizer para resolverlos y analizar el comportamiento de las soluciones. 


\subsection{Introducción}

El análisis de decisiones ha promovido el desarrollo y aplicación de diversas técnicas que posibilitan estudiar problemas complejos de decisión (Macharis et al., 2009). La complejidad de estos problemas se reviste de la variedad de criterios que tienen un impacto en la decisión y que generalmente son independientes, contradictorios y, en ocasiones, de difícil medición (Yannis et al., 2020). En el campo del transporte, por ejemplo, la construcción y operación de la infraestructura provoca cambios en casi todos los elementos del medioambiente (Broniewicz \& Ogrodnik, 2020), así como en la calidad de vida de la población (D’Eusanio et al., 2019). La toma de decisiones en este sector suele ser una tarea profunda y complicada, es por ello que en los análisis conviene tener en cuenta los criterios económicos, sociales, medioambientales, e inclusive, dependiendo de la decisión a tomar, criterios políticos (Camargo-Pérez et al., 2013).

El análisis de toma de decisiones tiene como objetivo priorizar, clasificar o seleccionar las alternativas de decisión en función de los criterios que se definan para evaluarlas y compararlas (Yannis et al., 2020). En el campo del transporte, entre las técnicas empleadas destacan las técnicas multi-atributo como AHP, ELECTRE, TOPSIS, DEMATEL o PROMETHEE (Wątróbski et al., 2019), pero también los modelos analíticos y de simulación son técnicas de extensa aplicación (Crainic et al., 2018; Elbert et al., 2020). Los modelos analíticos proporcionan un importante conjunto de herramientas de análisis, en especial, las técnicas de programación matemática son adecuadas para manejar el nivel de precisión requerido debido a la complejidad inherente en la valoración de los criterios que caracterizan los problemas (Arabani \& Farahani, 2012). La localización de instalaciones/hubs (Farahani et al., 2010; OrtizAstorquiza et al., 2018; Puerto et al., 2018), el diseño de redes de suministro (Govindan et al., 2017) y el diseño de redes urbanas de transporte (Jia et al., 2019) son algunos de los problemas en cuyo análisis de decisiones se han empleado las técnicas de programación matemática.

En el presente capítulo se explican los pasos que deben llevarse a cabo para aplicar las técnicas de programación matemática. En la sección 3.2. se describe la secuencia de pasos para formular los modelos. Siguiendo cuidadosamente cada uno de estos, en la sección 3.3. se proporciona una versión simple del problema de decisiones respecto a la descripción de la situación real presentada en el capítulo 2, en la sección 3.4. se detalla el modelado de los datos del problema y la estructura de la red de entrada para efectuar la interpretación matemática del problema combinado LI-DR, y en la sección 3.5. se describen los esquemas de resolución empleados para resolver y validar los modelos de programación que se proponen en esta tesis. Finalmente, las conclusiones de este capítulo se exponen en la sección 3.6.

\subsection{Técnica de análisis de decisiones: Programación matemática}

Un modelo de programación matemática, también entendido como de optimización, se compone de una o varias funciones objetivo que se establecen con el sentido de maximizar o minimizar, y que están sujetas a un conjunto de restricciones matemáticas que retratan las condiciones bajo las cuales se toman las decisiones (García-Sánchez, 2012; Mansouri et al., 2015). Un modelo de programación, de tipo lineal y mono-objetivo, se puede definir formalmente de la siguiente manera (García-Sánchez, 2012): 
Sujeto a:

$$
A_{i}(X) \approx b_{i} \quad i=1 \ldots m
$$

donde:

$X: \quad$ es el vector de variables

$f(X): \quad$ es la función objetivo

$A_{i}: \quad$ es la matriz de coeficientes

$\approx: \quad$ es el signo de la restricción $[\leq ;=; \geq]$

$b_{i}$ : $\quad$ es el vector de términos independientes

$A_{i}(X) \approx b_{i}:$ es el conjunto de restricciones, $i=1 \ldots m$

Como herramienta para el análisis en la toma de decisiones, las técnicas de programación matemática requieren la consecución de una serie de pasos que implican: a) la definición del problema, b) la construcción del modelo, c) la resolución del modelo, d) los ajustes y validación del modelo, y e) la implementación (Taha, 2012). La Figura 3.1. muestra la sistemática para la aplicación de estos pasos y la correspondencia de los agentes/actores que lideran el cumplimiento de cada paso.

Para García-Sabater \& Maheut (2015), Taha (2012) y Mathur \& Solow (1996) el alcance de cada paso debe estar bien delimitado. La definición del problema conduce a la comprensión del problema como un sistema a partir de la situación real, identificando las alternativas de solución, los objetivos y las limitaciones bajo las cuales funciona el sistema que se pretende modelar. En este paso es importante recabar la información de los decisores y/o grupos de interés relacionados con la solución al problema, tanto como en especificar las hipótesis o supuestos básicos concernientes al funcionamiento del sistema. El paso referido a la construcción del modelo conlleva a transformar el problema definido en un conjunto de relaciones matemáticas, es decir, la formulación del modelo de programación. Estas relaciones pueden adaptarse a una formulación estándar, o formularse nuevas relaciones. El conjunto de relaciones matemáticas determina el tipo de modelo de programación matemática. Por su parte, la resolución del modelo consiste en obtener la solución óptima, inclusive, cuando el modelo experimenta cambios en los parámetros. En este paso puede requerirse la aplicación de métodos o el desarrollo de algoritmos específicos, vinculando la codificación y programación informática de la formulación. Los ajustes y validación del modelo implican comprobar que la solución obtenida tenga sentido para el sistema estudiado. El último paso, el de implementación, reside en utilizar la solución por parte de los decisores o grupos de interés ${ }^{19}$.

\footnotetext{
${ }^{19}$ Debido a que el problema que se estudia en esta tesis tiene un alcance político para su implementación y no es inmediata la implementación de las decisiones, no es posible analizar el último paso respecto al impacto en la implementación de la solución.
} 
Figura 3.1. Sistemática para la aplicación de las técnicas de programación matemática

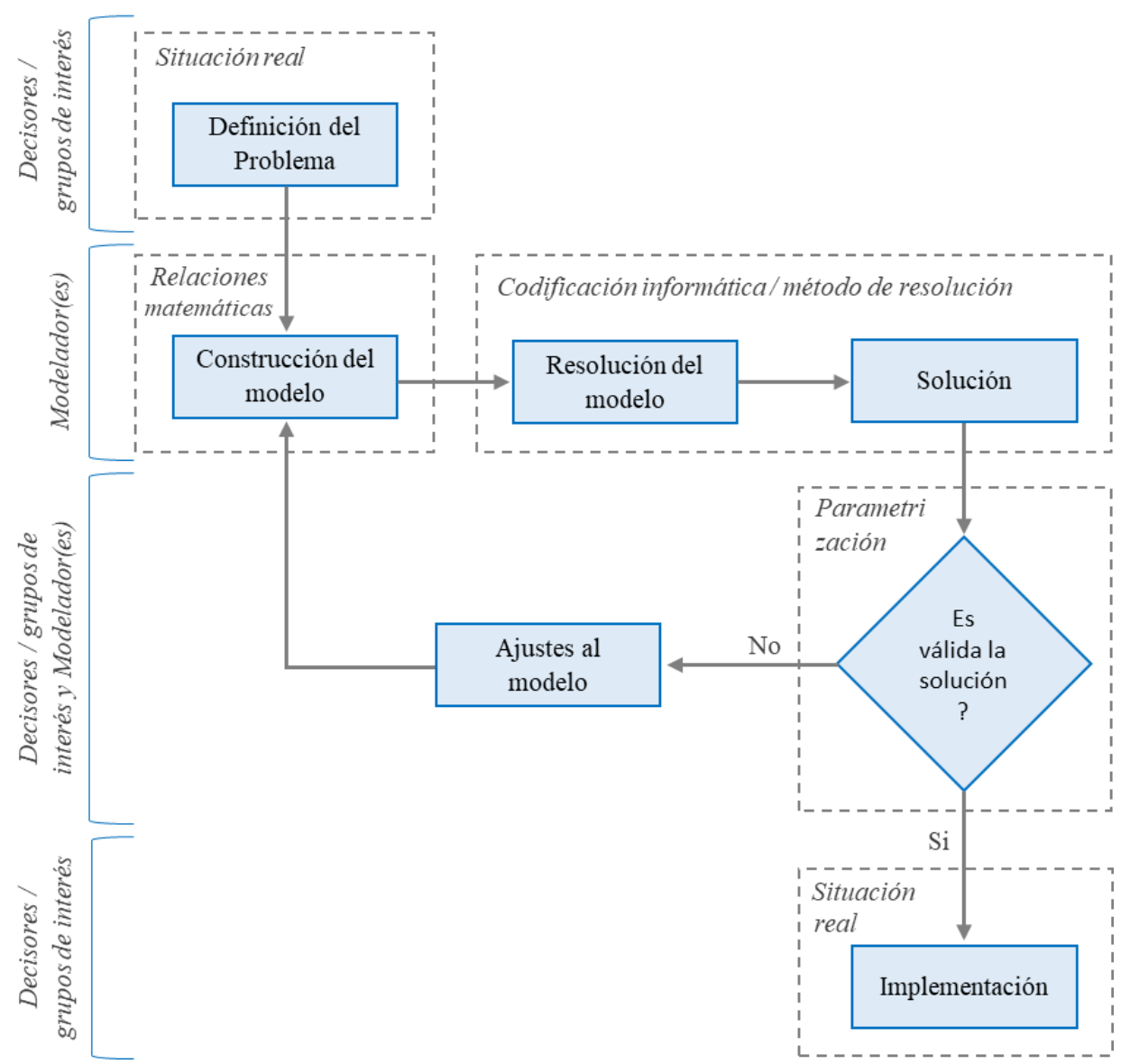

Fuente: Elaboración propia a partir de García-Sabater \& Maheut (2015) y Mathur \& Solow (1996)

Los modelos de programación pueden ser de tipo lineal, no lineal, entero, lineal enteromixto, estocásticos o difusos (Akgün \& Erdal, 2019; Mula et al., 2006) ${ }^{20}$. En general, estos modelos tienen una gran utilidad práctica porque, entre otras, no es indispensable obtener información de los decisores a lo largo de todo el proceso ya que los supuestos o hipótesis planteados en los primeros pasos de la sistemática compensan este requerimiento (Danesh et al., 2018). Igualmente, los modelos de programación matemática abarcan un número infinito o muy grande de posibles soluciones que no se conocen explícitamente al principio del proceso de formulación (Yannis et al., 2020), capturan las interdependencias entre los distintos criterios y alternativas de decisión que conducen a un análisis más eficaz de las decisiones (Jamshidi et al., 2019) y se adaptan fácilmente a la toma de decisiones dinámica y la formulación de variantes (Baykasoğlu \& Gölcük, 2019).

\subsection{Problema combinado LI-DR intermodal: Versión simple del problema real}

Con el propósito de eliminar las redundancias y ajustar la dimensión de la situación real a un problema tratable con las técnicas de programación matemática, en esta sección se define el

${ }^{20}$ En esta tesis no se profundiza en los tipos de programación debido a que las formulaciones que se proponen en los capítulos 5,6 y 7 son modelos de programación lineal entero-mixto. Para profundizar en los tipos de modelos se pueden consultar las revisiones de literatura propuestas por Agámez-Arias et al. (2021), Eskandarpour et al. (2015) y Sun \& Fan (2020). 
problema de decisiones planteando una versión simple de la situación real presentada en el capítulo 2. Por lo tanto, en la subsección 3.3.1. se delimita el problema y en la subsección 3.3.2. se presentan los criterios de optimización definidos para formular los modelos de programación que se proponen en esta tesis.

\subsubsection{Delimitación del problema}

Para Taha (2012): “es imperativo que se consideren primero los datos disponibles para luego definir el alcance del problema" (p. 9). Los datos son propiedades o atributos específicos que se utilizan en los criterios de optimización y en las especificaciones o relaciones del sistema que se modela (García-Sánchez, 2012). La variedad de aspectos económicos, sociales y medioambientales que caracterizan la situación real presentada en el capítulo 2 conducen a identificar distintos criterios, sin embargo, la disponibilidad de los datos o la imposibilidad de obtenerlos conllevan a valorar y elegir aquellos criterios más apropiados. Además de los datos citados en el capítulo 2, otros datos necesarios se complementaron con información suministrada por el IPREG y más concretamente por su director Julio Amézquita López ${ }^{21}$. En concordancia con lo anterior y evitando dar paso a la pérdida de representatividad de la situación real, se define la versión simple del problema de la siguiente manera.

El área de incidencia para el análisis de las decisiones se acota a una red de aprovisionamiento, pues se identifica que las principales necesidades de la ZODES radican en la infraestructura de transporte primario y la conexión entre las redes modales de transporte que confluyen en ella. Esta delimitación conduce a que se transforme el planteamiento inicial hacia un problema de diseño de red de transporte teniendo en cuenta las ventajas que ofrece el transporte intermodal. Para abordar el problema se detectan decisiones de nivel estratégico y táctico. En las decisiones estratégicas se reconoce la necesidad de localizar dos tipos de proyectos de inversión: infraestructura de primera milla de diferentes modos de transporte y terminales intermodales con distintas configuraciones modales. Tales decisiones, principalmente las de localización de infraestructura de primera milla, ponen de relieve la importancia de incorporar en el análisis las redes de transporte subyacentes para proponer alternativas de solución al diseño de la red de transporte intermodal. Por tanto, en las decisiones tácticas se revela la importancia en la vinculación de la asignación de la demanda a las terminales intermodales y las redes subyacentes.

Para establecer la red de aprovisionamiento se identifican los nodos de origen y destino. Los nodos de origen representan la ubicación desde la cual se puede satisfacer la demanda y los nodos de destino corresponden a la ubicación donde se debe satisfacer la demanda. El número y distribución atomizada de ofertantes tiene un impacto residual respecto a la localización de los proyectos de primera milla, por lo que se establece una agrupación de las unidades de producción agrícola (UPA) por municipios para fijar los nodos de origen. Siguiendo este mismo argumento, se identifican los nodos de destino según la agrupación de

\footnotetext{
${ }^{21}$ Julio Amézquita López, director del IPREG (Universidad de Cartagena, Colombia), ha participado en diversos proyectos de investigación y extensión a nivel gubernamental y regional que incorporan o se han centrado en el estudio de la ZODES Magdalena Medio; entre los más recientes trabajos está "La infraestructura logística en el sur de Bolívar: estado actual y potencialidades" (Amézquita-López et al., 2018). Igualmente, Julio ejerce como Acompañante Regional de la doctoranda para el desarrollo de esta tesis doctoral. La figura de Acompañante Regional es propia de las pautas establecidas por la Fundación CEIBA y la Gobernación de Bolívar (Colombia) para conceder a la doctoranda una Beca Condonable del programa de formación de talento humano "Bolívar Gana con Ciencia".
} 
distintos puntos de demanda en un área determinada del territorio nacional. Si bien la ZODES se caracteriza por una diversa estructura productiva, son 4 los productos que cuentan con mayor potencial de comercio y 3 de ellos pertenecen a un mismo grupo de alimentos, por lo tanto, se suman las cargas para introducirlas en el modelo como un único producto. Debido a que se presenta un exceso en la producción que se convierte en pérdidas para el agricultor, se consideran las pérdidas físicas para analizar los efectos en la solución óptima y su distribución entre los cultivadores.

En consecuencia, se identifica que el problema está relacionado con los denominados problemas combinados de localización de instalaciones-diseño de red (LI-DR). El objetivo esencial de este tipo de problemas es decidir las mejores localizaciones para la infraestructura, que ofrecen el mejor servicio a un conjunto de puntos de demanda, así como la localización o selección de los arcos de la red de transporte a través de los cuales se asigna la demanda (Contreras \& Fernández, 2012), generalmente, minimizando los costes (Farahani \& Hekmatfar, 2009). Este es un problema desafiante, complicado y matemáticamente complejo porque integra distintos niveles de decisión y grupos de interés (López-Ospina et al., 2021). La implementación de las decisiones estratégicas suscita requerimientos de inversión que se circunscriben a los actores gubernamentales de orden local y regional. En este sentido, la disponibilidad de financiación para efectuar las inversiones se reduce, convirtiéndose el presupuesto de inversión en una restricción para el diseño de la red intermodal.

Las particularidades económicas, sociales y medioambientales de la ZODES suscitan el interés de distintos grupos. Por ejemplo, la participación de actores gubernamentales en la toma de decisiones garantiza que la inversión también tenga un impacto social favorable, inclusive, avala la inversión proveniente de actores privados (Wiegmans \& Behdani, 2018). Para el problema combinado LI-DR intermodal, los intereses de los diversos grupos se reflejan en los criterios de optimización seleccionados. Estos criterios se exponen a continuación.

\subsubsection{Criterios de optimización}

Las revisiones de la literatura llevadas a cabo permitieron identificar los criterios de optimización $^{22}$ que sirven de referencia para valorar y seleccionar los más apropiados para brindar alternativas de solución al problema combinado LI-DR intermodal. Estos criterios integran las funciones objetivo de los modelos de programación matemática que se desarrollan en esta tesis. Para valorar y seleccionar los criterios económicos, sociales y medioambientales se generaron discusiones con los directores de tesis y el director del IPREG. De igual manera, se consideró el marco de indicadores para los objetivos de desarrollo sostenible (ODS) y las metas establecidas en la Agenda 2030 (Naciones Unidas, 2020). La Figura 3.2. muestra la correspondencia de los criterios de optimización seleccionados con los ODS. Otros resultados de las discusiones, tales como la participación del mercado a partir de las pérdidas físicas de producto, la interdependencia entre las decisiones de localización y los múltiples presupuestos de inversión, se plantean como condiciones para validar los modelos que se formulan y, por ende, extender el análisis a la solución óptima. En el apartado 3.3.2.1. se detallan los criterios económicos y en el apartado 3.3.2.2. los criterios sociales y medioambientales.

\footnotetext{
${ }^{22}$ Los resultados del análisis de la literatura se presentan en el capítulo 4 y en el capítulo 6 se listan los criterios sociales y medioambientales más representativos, sin embargo, un amplio listado de criterios puede consultarse en el anexo B.
} 
Figura 3.2. Criterios seleccionados para optimizar la solución del problema
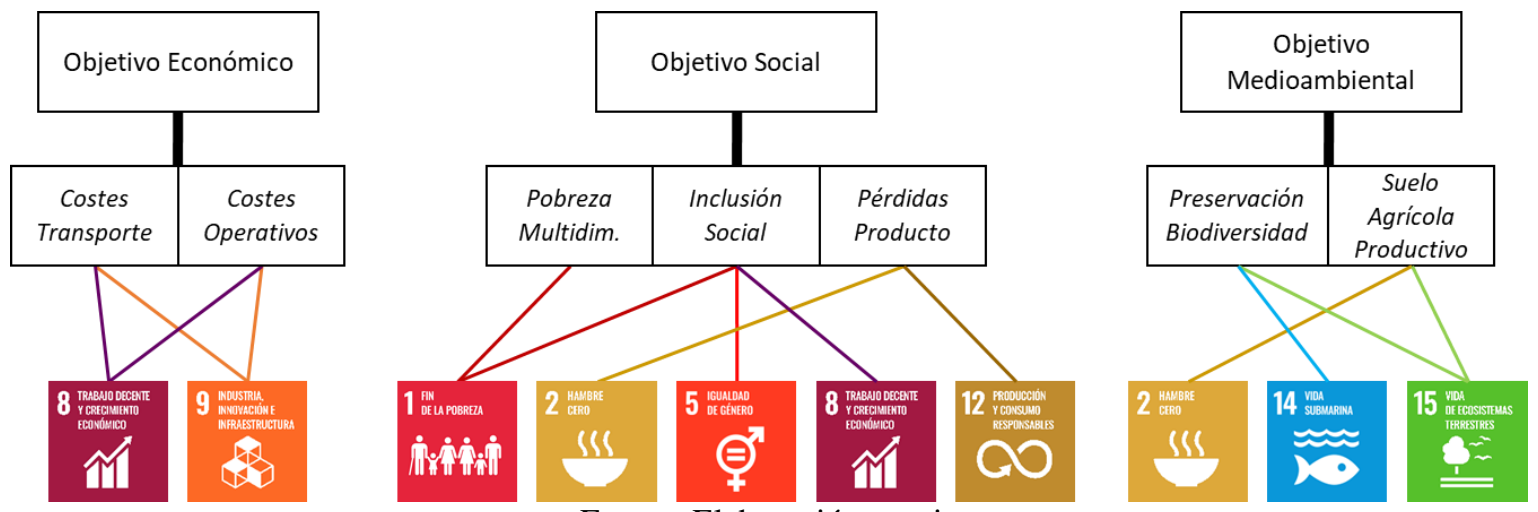

Fuente: Elaboración propia

\subsubsection{Criterios económicos}

Desde la dimensión económica del problema se seleccionan los costes de transporte y los costes operativos en las terminales intermodales para optimizar el problema combinado LI-DR intermodal. Los costes de transporte son vitales porque simulan el volumen de comercio, influyen en los patrones de flujo de toda la red y conducen a la diversificación de las actividades económicas en las áreas más próximas a los enclaves productivos (Berg et al., 2017). Los costes operativos determinan las conexiones más importantes para localizar las instalaciones, consolidar la carga (Kadaifci et al., 2019) y redirigirla a su destino asumiendo menores costes en el enrutamiento (Mostert et al., 2017). De acuerdo con la reducida disponibilidad de financiación para diseñar la red intermodal se invalida la posibilidad de optimizar los costes de inversión, por lo que estos se contemplan como una limitación a la solución del problema. Los grupos de interés esperan beneficios derivados de la reducción de otros costes logísticos ocasionados por el almacenamiento o las pérdidas de producto, pero la falta de registros o la dificultad en el cálculo y tratamiento unificado imposibilita la optimización de estos.

\subsubsection{2. $\quad$ Criterios sociales y medioambientales}

En las dimensiones social y medioambiental es usual que los criterios de optimización estén vinculados a los aspectos internos de las organizaciones, más aún cuando el problema de diseño de red es proclive a optimizar la red de la cadena de suministro (Bubicz et al., 2019; MorenoCamacho et al., 2019). No obstante, conforme a la situación socioeconómica de la ZODES parece más adecuado definir los criterios desde una óptica externa. Para la dimensión social se definen los criterios de pobreza multidimensional, inclusión social y pérdidas de producto. La pobreza multidimensional es medida por un índice que compagina la incidencia e intensidad de la pobreza a partir de un conjunto de "privaciones" relacionadas con la salud, la educación y el nivel de vida (Alkire \& Santos, 2014). La inclusión social se centra en la participación de la población en entornos sociales y comunitarios que mejoran la calidad de vida (Cordier et al., 2017), en base al acceso educación, empleo, servicios y otros (Litman, 2019). En la cadena de suministro agroalimentaria las pérdidas de producto $^{23}$,especialmente las que ocurren en las

\footnotetext{
${ }^{23}$ Nourbakhsh et al. (2016) afirma que en las cadenas de suministro agroalimentaria las pérdidas de producto corresponden a la degradación, en calidad y cantidad, de un producto, y pueden ocurrir desde el lugar donde se cosecha hasta el lugar de consumo.
} 
zonas de cultivo, no han recibido tanta atención (Shukla \& Jharkharia, 2013), por tanto, este criterio supone una gran oportunidad para esta tesis y la literatura.

La geografía de la ZODES favorece la estructura productiva agrícola y garantiza la oferta de servicios medioambientales esenciales para la continuidad de la especie humana; la extensión de los bosques naturales y la degradación de estas áreas conllevan a concebir la dimensión medioambiental desde la conservación y preservación. Por tanto, se procura la transformación del territorio de acuerdo con la preservación de la biodiversidad y el suelo agrícola productivo. La inversión en infraestructura fomenta el crecimiento económico de las regiones (Witte et al., 2014), pero también conduce a una fragmentación y pérdida continuada de los ecosistemas y sus especies (Ortega et al., 2014). Los cultivos agrícolas son una fuente significativa de degradación del suelo debido al uso de fertilizantes y pesticidas (Oren et al., 2004). Esta degradación impacta directamente en la disminución o pérdida de los atributos físicos, químicos (nutrientes), biológicos (reservas de carbono) y ecológicos (funciones de los ecosistemas) del suelo (Lal, 2015). Es por ello que la planificación de los nuevos trazados en las redes de transporte debe orientarse hacia una gestión sostenible del territorio (ÁlvarezMiranda et al., 2019).

\subsection{Modelado del problema combinado LI-DR intermodal para construir los modelos de programación matemática}

De acuerdo con el problema de decisiones definido en la sección 3.3. la programación matemática podría formularse directamente como un modelo multi-objetivo. Sin embargo, con la finalidad de analizar y validar los efectos desde lo económico, social y medioambiental del problema combinado LI-DR intermodal en el diseño estratégico-táctico de la red de transporte intermodal, se realiza una construcción gradual de la formulación. Por tanto, se proponen 2 versiones que se representan en 5 variantes, tal y como se muestra en la Figura 3.3.

Para la construcción de las formulaciones es indispensable que primero se modele el problema de decisiones. Por ello, en esta sección se detalla el modelado del problema combinado LI-DR intermodal, mientras que, los supuestos básicos y formulaciones se presentan en los capítulos 5, 6 y 7. En la subsección 3.4.1. se modelan los datos del problema $\mathrm{y}$, conforme con las características del problema combinado LI-DR intermodal, también se modela la estructura de la red de entrada. Para construir los modelos es aconsejable verificar si algunas de las relaciones matemáticas se pueden referenciar de una formulación previamente validada o construir nuevas relaciones (Taha, 2012). Siguiendo esta postura se presenta en la subsección 3.4.2. la formulación propuesta por Amaral et al. (2012), cuyas relaciones matemáticas se utilizan como referente para formular los modelos de programación que se desarrollan para explorar el problema de decisiones. 
Figura 3.3. Versiones y variantes de los modelos de programación propuestos en esta tesis doctoral

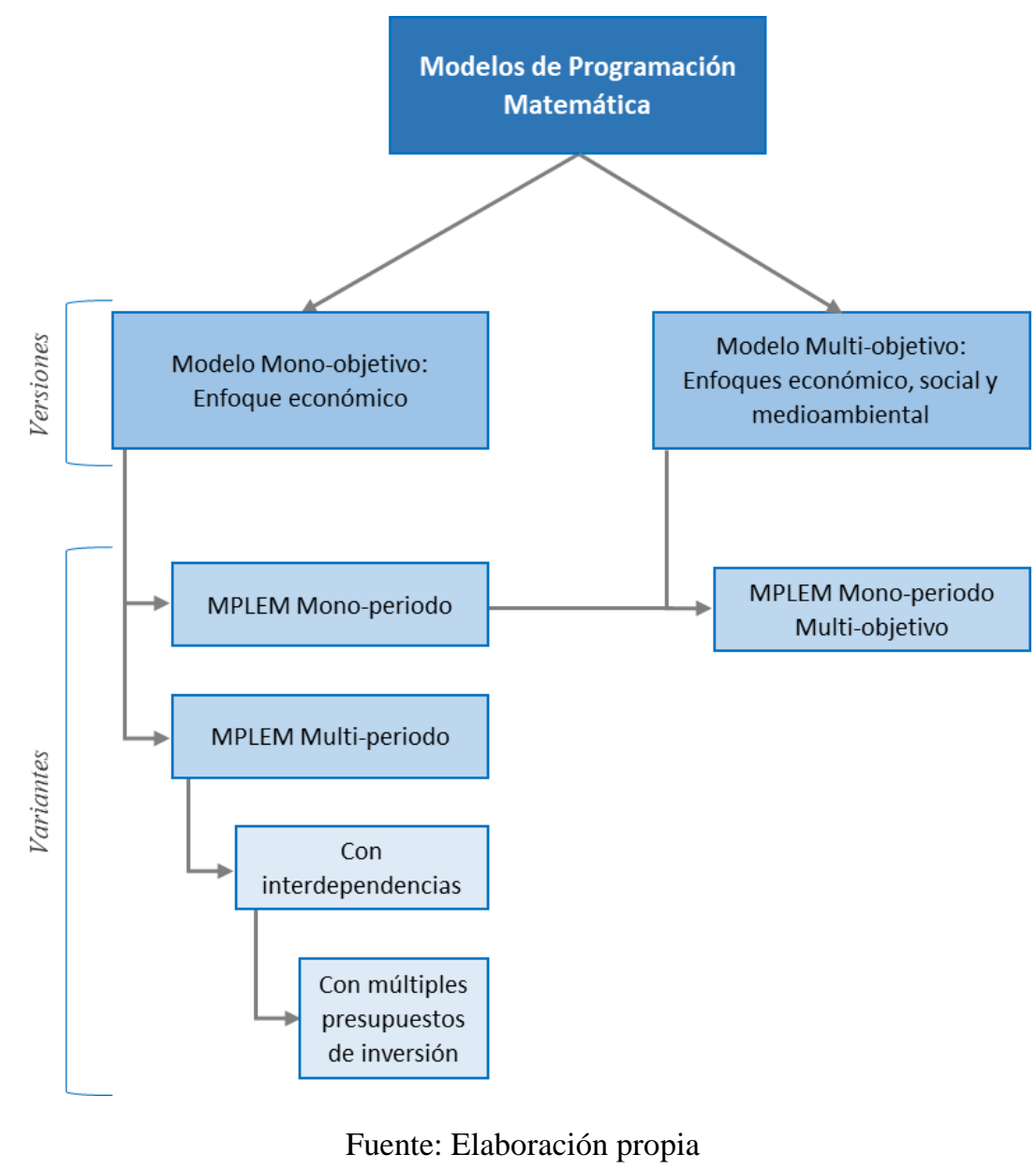

\subsubsection{Modelado de la versión simple del problema}

La limitación en la financiación para diseñar la red intermodal y los altos costes de construcción que suponen los tipos de proyectos identificados en la subsección 3.3.1. (infraestructura de primera milla y terminales intermodales), han supuesto fraccionar la construcción de los proyectos proporcionando distintas alternativas. Para ello, a la decisión de localización se vincula una nueva decisión estratégica relacionada con la alternativa de capacidad para las terminales intermodales, y con la alternativa vial para la infraestructura de transporte de primera milla. Por otra parte, la incorporación de la estructura de las redes de transporte subyacentes y las características de conectividad en la ZODES admiten que la decisión táctica de asignación de la demanda sea múltiple y que ésta quede supeditada a los flujos en cada nodo y arco de la red. Los flujos en las terminales intermodales no pueden superar la capacidad de la alternativa elegida, mientras que, los flujos en los arcos modales no están limitados a un nivel máximo. Las pérdidas de producto se modelan como una decisión para analizar los efectos en la solución. En la Tabla 3.1. se detallan las decisiones del problema combinado LIDR intermodal ${ }^{24}$.

\footnotetext{
${ }^{24}$ La nomenclatura matemática de índices, parámetros y variables de decisión empleada en la construcción de las distintas variantes de los modelos de programación se detallan en los capítulos 5, 6 y 7 .
} 
Tabla 3.1. Decisiones del problema combinado LI-DR intermodal

\begin{tabular}{l}
\hline Decisiones \\
$\begin{array}{ll}\text { Estratégicas } \\
1 & \text { Localización y alternativa de capacidad de la terminal intermodal (sí / no) } \\
2 & \text { Localización y alternativa vial de transporte de primera milla (sí / no) } \\
\text { Tácticas } & \text { Asignación de los flujos en la red (cantidad) } \\
1 & \text { Ptras decisiones } \\
1 & \text { Pérdidas de producto (cantidad) }\end{array}$ \\
\hline
\end{tabular}

La Figura 3.4. muestra la red de entrada que se utiliza como base para analizar las soluciones al problema combinado LI-DR intermodal. Por una parte, se tienen los nodos de origen, nodos de destino y terminales intermodales. Los nodos de origen y destino se proporcionan como conjuntos de nodos diferentes. Los primeros, se concentran en la ZODES, mientras que, los segundos están dispersos en distintas zonas del territorio nacional. Las terminales intermodales se localizan entre los nodos de origen y los nodos de destino. La asignación del flujo entre los nodos de origen y las terminales intermodales, así como entre los terminales intermodales y los nodos de destino se establece con una única dirección. La asignación está sujeta a la ordenación de las redes de transporte subyacentes. Es decir, una terminal puede ser servida por cada nodo de origen si existe conexión a través de una red modal, así mismo, cada terminal puede servir un nodo de destino siempre que tenga conexión al menos con una red modal.

Por otra parte, se cuenta con las redes de transporte subyacentes. Cada red modal subyacente está compuesta por nodos y arcos. Los nodos, por ejemplo, como los que se muestran en la Figura para la red de carreteras, responden a las intersecciones de dos o más vías; los arcos tienen características unidireccionales o bidireccionales y corresponden a las vías. Las secciones de las redes subyacentes que se encuentran entre los nodos de origen y las terminales intermodales pueden requerir la localización de los proyectos de primera milla si se decide asignar el flujo a través de estos. Las redes subyacentes son de los modos por carretera, fluvial y ferroviario. Aunque los tramos de estas redes modales pueden ser de orden primario, secundario o terciario ${ }^{25}$, no se considera explícitamente las categorías para modelar la red de entrada sino que se contemplan las distinciones a través de los costes de transporte. Es decir, los costes en los tramos de orden terciario son mayores que en los tramos de orden primario, además, los costes de transporte también se distinguen según el modo.

\footnotetext{
${ }^{25}$ Ibíd 16.
} 
Figura 3.4. Red de entrada para el problema combinado LI-DR intermodal

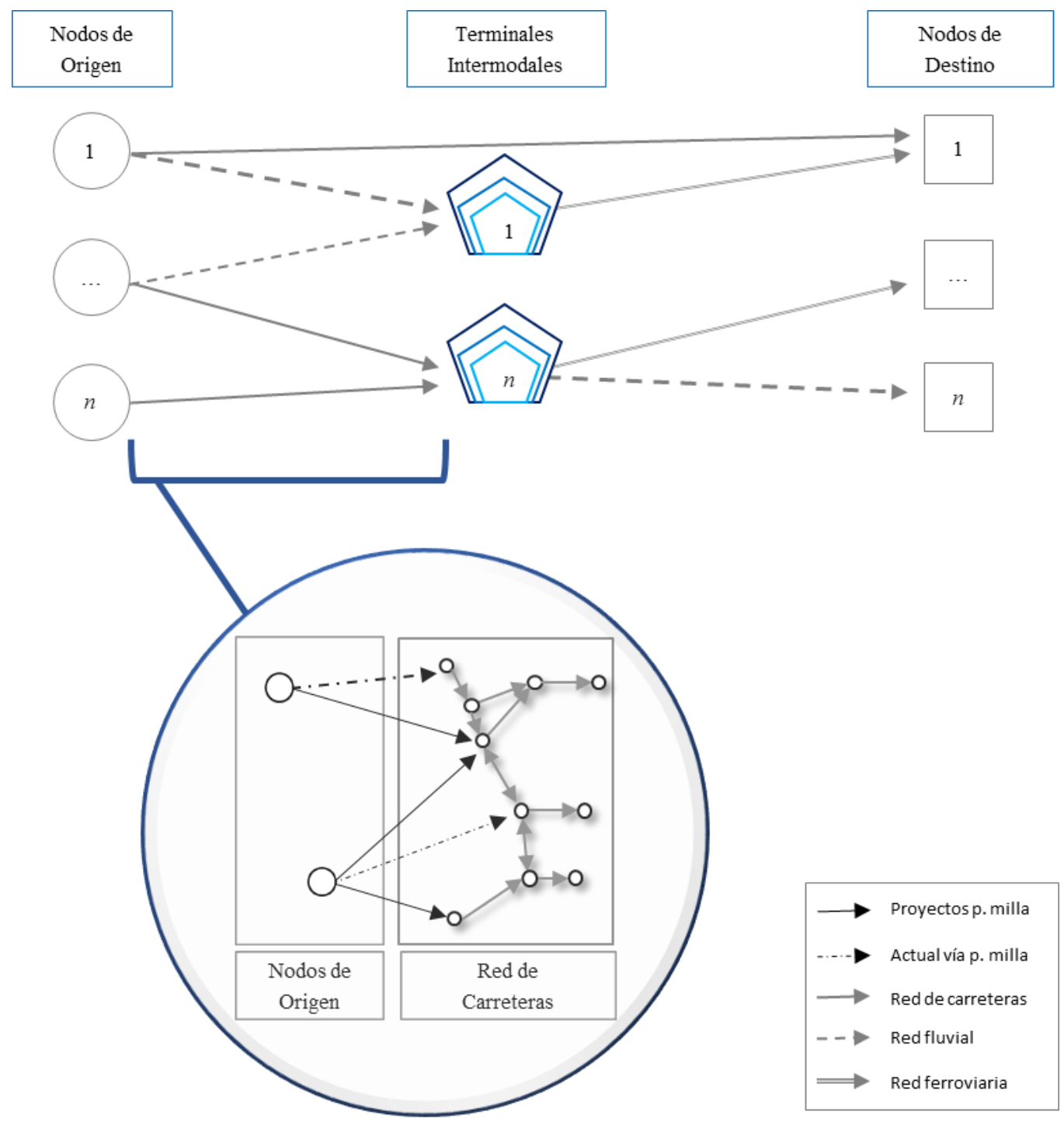

Fuente: Elaboración propia

En la Tabla 3.2. se describen los datos y estructura de la red de entrada del problema combinado LI-DR intermodal. La red de entrada se modela como un grafo dirigido $G=(N, A)$ donde $N$ son los nodos y $A$ los arcos de la red ${ }^{26}$. Debido a que las terminales intermodales son los puntos estratégicos donde se conectan al menos dos redes modales de transporte, estas terminales no se modelan como nodos sino como arcos y son definidas como arcos intermodales. Para localizar los arcos de primera milla y los arcos intermodales se proponen tres alternativas de decisión, esto indica que este grupo de arcos se incorporan por triplicado a la estructura de la red.

\footnotetext{
${ }^{26}$ En el capítulo 5 se precisa la nomenclatura de la estructura de la red de entrada.
} 
Tabla 3.2. Estructura de la red de entrada del problema combinado LI-DR intermodal

\begin{tabular}{ll}
\hline Datos de la red de entrada \\
\hline 6 & Nodos de origen \\
5 & Nodos de destino \\
10 & Proyectos de infraestructura de transporte de primera milla (Arcos de primera milla) \\
7 & Proyectos de terminales intermodales (Arcos intermodales) \\
3 & Alternativas de localización de cada tipo de proyecto \\
3 & Redes modales subyacentes: carretera, fluvial y ferroviaria \\
\hline Estructura de la red de entrada \\
\hline 30 & Nodos \\
90 & Arcos \\
\hline
\end{tabular}

\subsubsection{Formulación de referencia}

La literatura ofrece un conjunto de formulaciones que se pueden utilizar como punto de partida para construir la programación matemática del problema combinado LI-DR intermodal que se estudia en esta tesis, por lo que se identifica la formulación propuesta por Amaral et al. (2012). Esta formulación presenta cierto grado de correspondencia respecto a la estructura de diseño de la red de transporte intermodal que aborda, las decisiones de localización de terminales intermodales y el modelado de las redes de transporte subyacente. Además, conserva similitud con algunos aspectos del problema que se estudian en esta tesis, en concreto, con el diseño de una red intermodal de aprovisionamiento. En la Tabla 3.3. se detalla la nomenclatura utilizada por Amaral et al. (2012) para representar la formulación que se presenta posteriormente.

Tabla 3.3. Nomenclatura utilizada por Amaral et al. (2012) para representar la formulación matemática

\begin{tabular}{ll}
\hline Conjuntos \\
\hline$O$ & Conjunto de vértices que representan puntos de oferta \\
$D$ & Conjunto de vértices que representan puntos de demanda \\
$V_{m}$ & Conjunto de vértices que representan los puntos de cada red modal, $m=1,2,3$ \\
$A_{m}$ & Conjunto de arcos de cada red modal, $m=1,2,3$ \\
$A^{\prime}$ & Conjunto de arcos que conectan los vértices de oferta y demanda a la red modal \\
$T$ & Conjunto de arcos candidatos para la instalación de terminales intermodales \\
\hline
\end{tabular}

\section{Parámetros}

$a_{i} \quad$ Cantidad ofrecida $(t)$ por el punto $i,\{i \in O\}$

$b_{j} \quad$ Cantidad demandada $(t)$ por el punto $j,\{j \in D\}$

$P \quad$ Número de terminales intermodales a instalar 


\begin{tabular}{ll}
\hline$c_{i, j}$ & Coste unitario de transportar la carga por el $\operatorname{arco}(i, j),\{(i, j) \in A\}$ \\
$u_{i, j}$ & Capacidad para el flujo de los $\operatorname{arcos}(i, j)$ de transbordo $(t),\{(i, j) \in T\}$ \\
\hline Variables de decisión \\
\hline$y_{i, j} \quad 1$ si se instala una terminal intermodal en $(i, j),\{(i, j) \in T\} ; 0$ en caso contrario \\
$X_{i, j} \quad$ Flujo del arco $(i, j),\{(i, j) \in A\}$ \\
\hline
\end{tabular}

$$
\min \sum_{(i, j) \in A} c_{i j} x_{i j}
$$

Sujeto a:

$$
\begin{array}{cl}
\sum_{(i, j) \in T} y_{i j}=P & \\
\sum_{j \mid(i, j) \in A_{O}^{\prime}} x_{i j} \leq a_{i} & \forall i \in O \\
\sum_{i \mid(i, k) \in A} x_{i k}=\sum_{j \mid(k, j) \in A} x_{k j} & \forall k \in V /\{O \in D\} \\
\sum_{i \mid(i, j) \in A_{1 D}^{\prime} \cup A_{2 D}^{\prime} \cup A_{3 D}^{\prime}} x_{i j}=b_{j} & \forall j \in D \\
x_{i j} \leq u_{i j} y_{i j} & \forall(i, j) \in T \\
x_{i j} \geq 0 & \forall(i, j) \in A \\
y_{i j} \geq\{0,1\} & \forall(i, j) \in T
\end{array}
$$

El modelo propuesto es una programación lineal entero-mixto (MPLEM). La función objetivo (3.3) minimiza los costes de transporte y de transbordo. Las restricciones (3.4) aseguran que se instalen exactamente $p$ terminales intermodales. Las restricciones (3.5), (3.6) y (3.7) garantizan el flujo en el sistema, refiriéndose las primeras a la oferta, las segundas a la conservación de los flujos, y las últimas a la demanda. Las restricciones (3.8) permiten el flujo solamente en las terminales intermodales abiertas, respetando las limitaciones de máxima capacidad en las mismas. Finalmente, las restricciones (3.9) y (3.10) definen los dominios de las variables de decisión.

Esta formulación se utiliza como referencia para construir el MPLEM mono-periodo con enfoque económico del problema combinado LI-DR intermodal. Para ello se adapta la función objetivo de acuerdo con los criterios de optimización descrito en la subsección 3.3.2., y respecto a las restricciones de la formulación, se consideran y ajustan las relaciones matemáticas representadas en las restricciones (3.5) a (3.10). La restricción (3.4.) se excluye 
de la formulación puesto que en el problema combinado LI-DR intermodal el número de decisiones de localización de nueva infraestructura está limitado por el presupuesto de inversión. Las demás variantes descritas en la Figura 3.3. se construyen a partir del MPLEM mono-periodo con enfoque económico. Los supuestos básicos y los modelos de programación matemática propuestos para cada una de las variantes que se analizan en esta tesis, se presentan en los capítulos 5, 6 y 7.

\subsection{Resolución y validación}

Para realizar el análisis de decisiones de los modelos de programación que se desarrollan en esta tesis, se implementan dos esquemas de resolución. En estos esquemas se utilizan los lenguajes de programación R y Python, y una licencia académica del software de optimización matemática Gurobi Optimizer para resolverlos y analizar el comportamiento de las soluciones. La selección del software Gurobi Optimizer se debe a sus ventajas en términos de versatilidad, flexibilidad, capacidad y tiempos de ejecución frente a otros solucionadores (Badyal et al., 2020; Liu \& Kwon, 2020). La licencia académica utilizada corresponde a la versión 8.1.0 y se ejecuta en una computadora portátil con CPU Intel Core i7 de $2.80 \mathrm{GHz}, 8 \mathrm{~GB}$ de RAM y Windows 10.

En las subsecciones 3.5.1. y 3.5.2. se describen los dos esquemas de resolución implementados y en la subsección 3.5.3. se presenta una descripción sobre cómo se lleva a cabo el análisis de las soluciones.

\subsection{1. $R+$ Gurobi}

Con el esquema de resolución $R+$ Gurobi se implementan los modelos de programación matemática haciendo uso del lenguaje $\mathrm{R}$ y el solucionador Gurobi Optimizer a través de la API $\mathrm{R}$ Interfaces en el entorno RStudio 1.1.456. Este esquema de resolución se utiliza para resolver los modelos cuyas variantes son programaciones mono-periodo, los cuales corresponden al MPLEM con enfoque económico que se presenta en el capítulo 5 y al MPLEM multi-objetivo del capítulo 7.

Para implementar el modelo de programación en el lenguaje $\mathrm{R}$ se hace necesario declarar los componentes del modelo conforme a la estructura establecida en el Manual de Referencia de Gurobi Optimización (2019). Los componentes corresponden a las librerías, datos del modelo, construcción del modelo, parámetros del modelo, y el solucionador y resultados del modelo. En la construcción del modelo se emplea un conjunto de argumentos a través de los cuales se describe la formulación propuesta para la programación matemática. Los argumentos para construir los MPLEM son los siguientes:

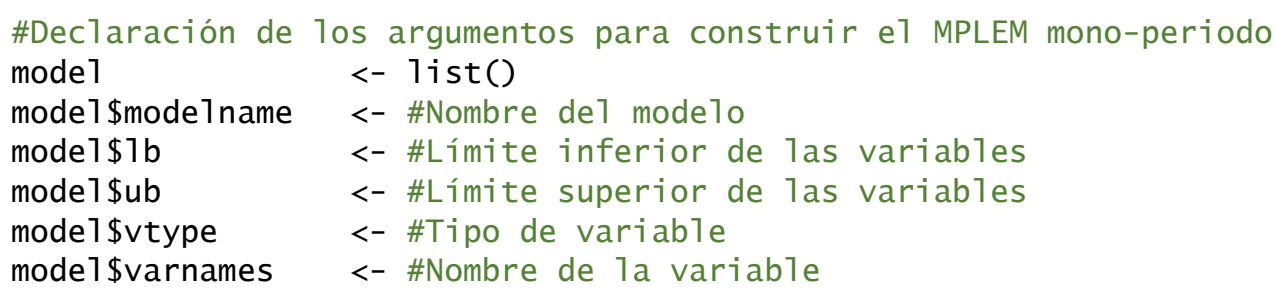




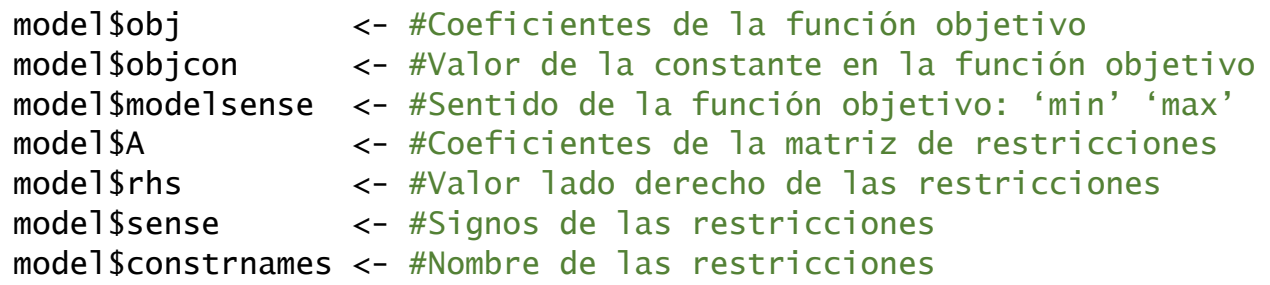

Para el MPLEM multi-objetivo se declaran los mismos argumentos anteriormente relacionados, con excepción del argumento mode1\$obj que se reemplaza por los siguientes argumentos:

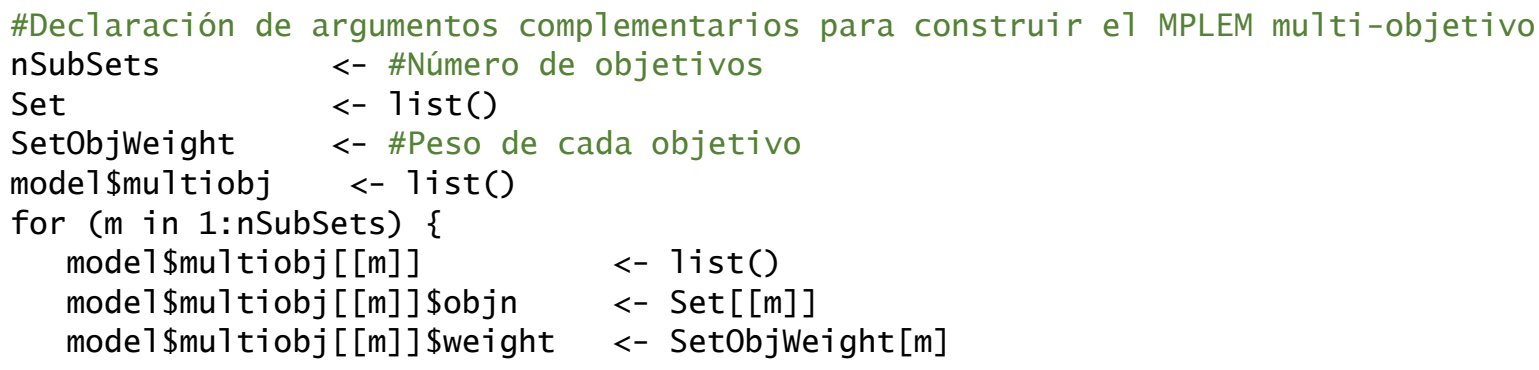

Los argumentos del modelo se declaran siguiendo las pautas de indexación con rutinas para generar matrices. Una matriz es una agrupación de elementos que está constituida por $n$ filas y $m$ columnas, en la cual la indexación del elemento $a_{i, j}$ representa la entrada $a$ ubicada en la fila $i$ y columna $j$. Para una interpretación de los elementos $a_{i, j}$ en el modelo de programación definido en la sección 3.2., se desagrega a continuación esta formulación.

$$
\min / \max C_{1} X_{1}+C_{2} X_{2}+C_{3} X_{3}+C_{m} X_{m}
$$

Sujeto a:

$$
\begin{aligned}
& a_{11} X_{1}+a_{12} X_{2}+a_{13} X_{3}+a_{1 m} X_{m} \approx b_{1} \\
& a_{21} X_{1}+a_{22} X_{2}+a_{23} X_{3}+a_{2 m} X_{m} \approx b_{2} \\
& a_{n 1} X_{1}+a_{n 2} X_{2}+a_{n 3} X_{3}+a_{n m} X_{m} \approx b_{n}
\end{aligned}
$$

La entrada de los elementos $a_{i, j}$ pueden ser nulos o no nulos, y se especifican en el argumento mode1\$A. Para identificar los elementos de la matriz se toma como base la estructura de red modelada en la subsección 3.4.1. Cada fragmento del grafo $G=(N, A)$ se convierte en variables de decisión que luego facilitan la construcción de la matriz A. En la Figura 3.5. se visualiza esta conversión para un fragmento del grafo en el que se conectan los nodos de origen con los nodos de la red de carreteras. La Figura 3.5.a. muestra las conexiones reales para los dos grupos de nodos mencionados. La Figura 3.5.b. representa las variables que 
se generan y las entradas nulas y no nulas en la matriz A para ese mismo fragmento del grafo. Si bien se generan variables de decisión para todas las posibles conexiones entre los nodos de origen y el nodo de carretera $\mathrm{NC} 1$, las conexiones reales se convierten en entradas no nulas. Cada entrada no nula constituye un arco de la red sobre el cual se puede obtener una decisión, que para el problema combinado LI-DR intermodal reflejan decisiones de localización y/o asignación.

Figura 3.5. Esquema construcción de la Matriz A

a.

Conexión real entre los Nodos de Origen y

Nodos de la red de Carreteras

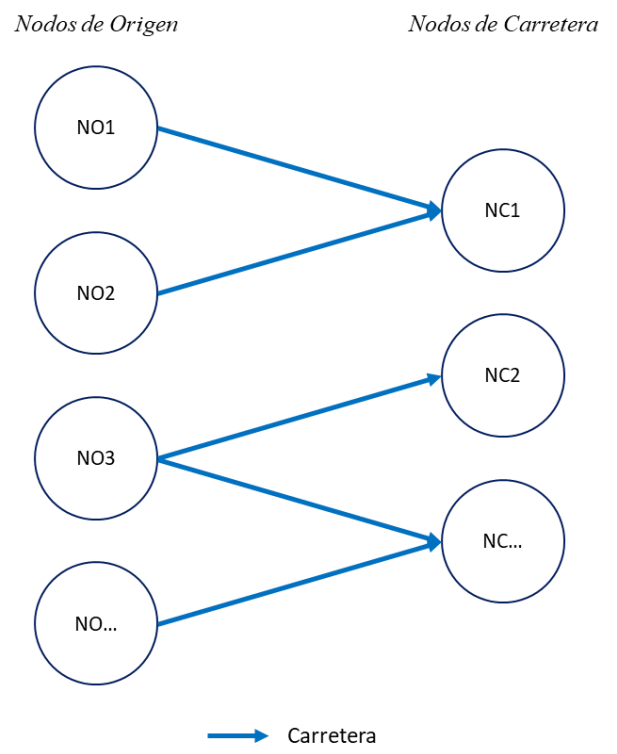

b.

Variables de decisión generadas para la matriz A: Entradas nulas y no nulas

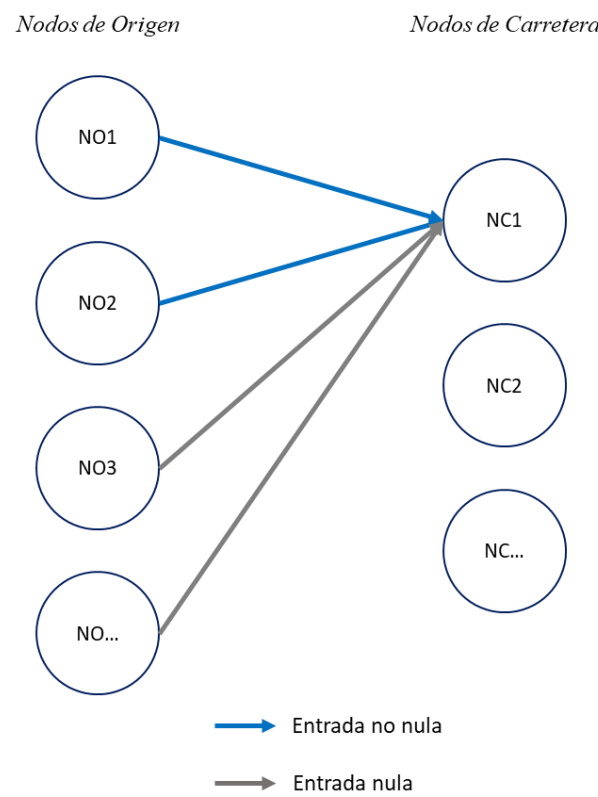

Fuente: Elaboración propia

Aunque las pautas de indexación con rutinas para generar matrices pueden derivar en un procedimiento tedioso, propenso a errores y frágil respecto a los cambios en el modelo matemático, la matriz construida permite mapear eficientemente las variables de decisión (Dunning et al., 2017). Las características de conectividad de la zona de estudio facilitan la construcción de la matriz A, la cual se construye generando submatrices para cada fragmento del grafo $G=(N, A)$. Estas submatrices se incorporan de manera agregada al argumento mode1\$A. Como ningún nodo del grafo tiene conexión con todos los demás nodos del grafo, el número de elementos no nulos introducidos es reducido. La matriz A se compone de 2.912 variables de decisión, 183 restricciones y 929 entradas no nulas. La Figura 3.6. muestra las entradas no nulas para las primeras 210 variables de decisión y 35 restricciones de la matriz $\mathrm{A}^{27}$. Esta sección de la matriz compila las decisiones de asignación de la demanda desde los nodos de origen hacia los nodos de las redes subyacentes por carretera y fluvial.

${ }^{27}$ En el anexo C se presenta la matriz A construida para resolver las variantes de los modelos de programación mono-periodo. 
Figura 3.6. Submatriz de A con entradas no nulas para las variantes de los modelos de programación mono-periodo

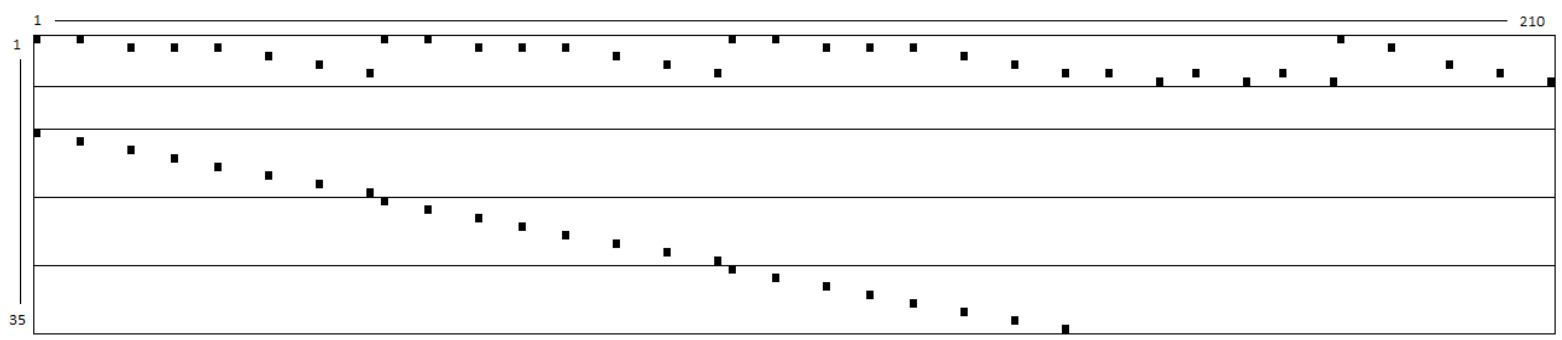

Fuente: Elaboración propia

Por otra parte, para una mejor disposición, colección y lectura de los datos de entrada y los datos de salida de cada instancia resuelta con este esquema, se conecta el entorno RStudio con el gestor de base de datos MySQL.

\subsubsection{Python + Gurobi}

Con el esquema de resolución Python + Gurobi se implementan los modelos de programación matemática con el lenguaje Python, haciendo uso del modelador PuLP, la interfaz gráfica Geany 1.36.0. y el solucionador Gurobi Optimizer. Este se utiliza para resolver los modelos cuyas variantes son los MPLEM multi-periodo que se desarrollan en el capítulo 6.

Para implementar un modelo de programación bajo este esquema de resolución se declaran las librerías, los datos de entrada, el modelo de programación, el solucionador y resultados del modelo (García-Sabater, 2021). En la declaración del modelo es necesario crear primeramente la variable prob para contener la formulación, por lo que la función objetivo y las restricciones se conectan a través de esta variable de la siguiente manera:

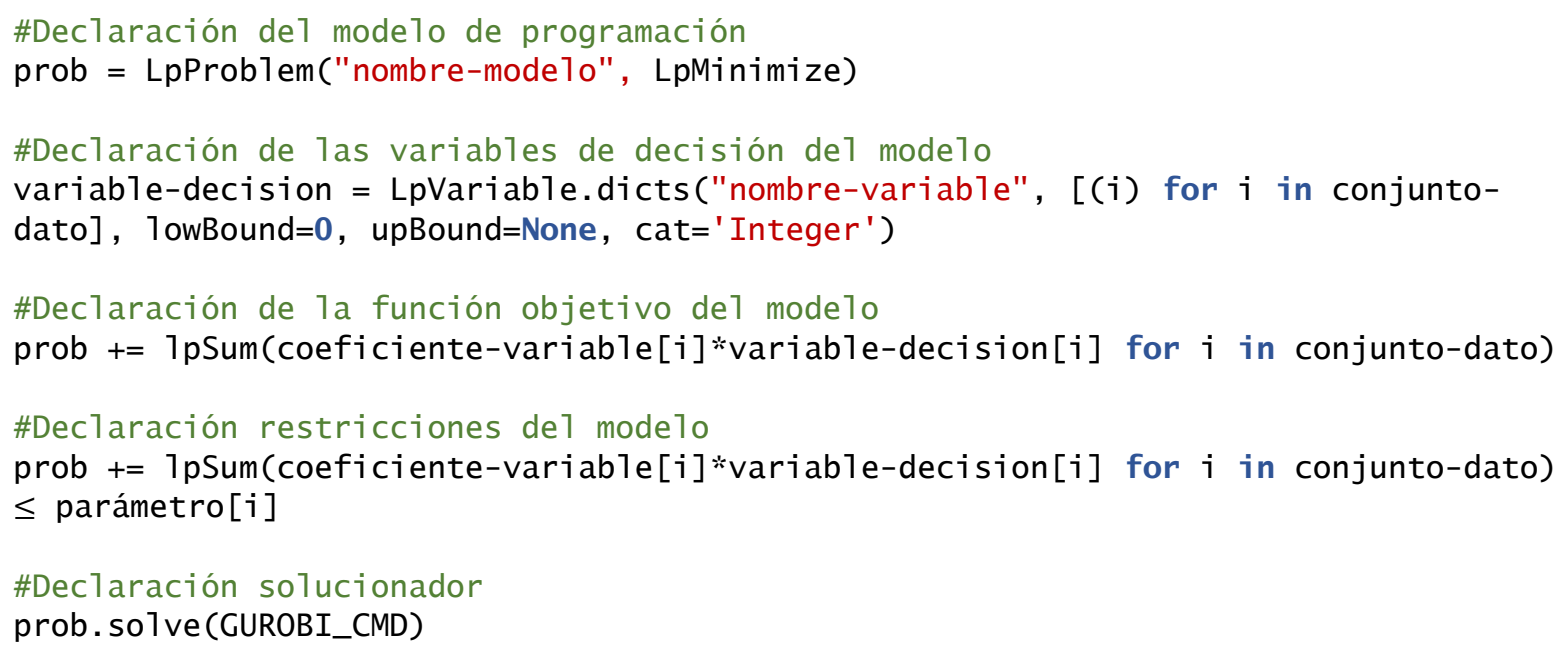

Para la construcción de cada declaración del modelo se programan secuencias de instrucciones basadas en iteraciones y condicionales. Para ello, se diseñan bucles con for, if, e if else, y se utilizan listas, tuplas y diccionarios para almacenar la secuencia de datos del 
modelo. Para este esquema de resolución, el grafo $G=(N, A)$ se proporciona como una secuencia de datos, en el cual cada dato corresponde a un par ordenado de nodos que equivale a un arco de la red. Por tanto, y en contraposición con el mecanismo de generación de la matriz A explicado en la subsección anterior, en el presente esquema solo se registran aquellos arcos existentes en la red de entrada. El número de variables de decisión y restricciones que se generan para cada variante multi-periodo se especifican en la Tabla 3.4.

Tabla 3.4. Variables de decisión y restricciones generadas en las variantes multi-periodo de los modelos de programación ${ }^{28}$

\begin{tabular}{l}
\hline Variantes multi-periodo \\
\hline MPLEM multi-periodo \\
$960 \quad$ Variables de decisión \\
$2246 \quad$ Restricciones
\end{tabular}

MPLEM multi-periodo con interdependencias

1110 Variables de decisión

8546 Restricciones

MPLEM multi-periodo con interdependencias y múltiples presupuestos de inversión

1770 Variables de decisión

15541 Restricciones

Para la lectura de los datos de entrada y la ordenación de los datos de salida de cada instancia que se resuelve, se utilizan ficheros csv.

\subsubsection{Análisis de la solución}

En la resolución de un modelo de programación se intenta diseñar la mejor solución considerando las interacciones entre las restricciones y la función objetivo o funciones objetivo (Mansouri et al., 2015). Cuando se dispone de una programación de objetivo único la resolución procura dar el mejor valor, el óptimo, a la única función objetivo del modelo (Bradley et al., 1977). Por el contrario, en la programación multi-objetivo no existe una única solución que optimice simultáneamente todas las funciones objetivo, el concepto de óptimo se reemplaza por el de eficiencia de Pareto cuyas soluciones se determinan como Pareto-óptimas (Mavrotas, 2009). Sin embargo, para el análisis de decisiones no basta solamente con encontrar la solución óptima o las soluciones Pareto-óptimas. En este es de interés analizar los efectos en la solución a partir de distintos escenarios, bien sea para realizar ajustes al modelo de programación o para evaluar escenarios en la implementación real de la solución.

\footnotetext{
${ }^{28}$ Este esquema de resolución, a modo de prueba, también se implementa para los modelos de programación cuyas variantes son los modelos mono-periodo y se determina que el número de variables de decisión generadas se reduce a 140.
} 
El análisis en el comportamiento de la solución consiste en efectuar cambios en los valores de los parámetros, ya sea en la función objetivo o en el lado derecho de las restricciones, para ir descubriendo los efectos y comportamiento en la solución. Este es un método ampliamente utilizado, por ejemplo, en Badyal et al. (2020), Correia \& Melo (2017), Kumar \& Mishra (2018), Munim \& Haralambides (2018), y Peng et al. (2019). No obstante, no responde a un procedimiento estándar a través del cual se deriven todos los posibles efectos que permitan a los tomadores de decisión obtener un análisis consolidado, sino que se deben generar un número determinado de instancias en las cuales se combinan los cambios a los valores de los parámetros. De este modo, se podrán generar instancias que permitan realizar el análisis de la solución en relación con la función objetivo, por ejemplo, considerando variaciones en los costes de transporte; con los recursos que restringen la solución, considerando variaciones en los valores de demanda, las capacidades y el presupuesto de inversión disponible; y combinando los cambios en los parámetros que inciden en la función objetivo y en el lado derecho de las restricciones.

El análisis de la solución se emplea en todos los modelos de programación que se proponen en esta tesis. Los cambios en los valores de los parámetros y el número de instancias generadas se presentan en los respectivos capítulos.

\subsection{Conclusiones}

En este capítulo se explicó el enfoque metodológico llevado a cabo para aplicar la programación matemática como técnica de análisis de decisiones del problema de investigación. Para ello, se identificó la secuencia de pasos para la aplicación de estas técnicas: definición del problema, construcción del modelo, resolución del modelo, ajustes y validación del modelo, y la implementación. En el paso de definición del problema se hizo necesario ajustar la dimensión de la situación real presentada en el capítulo 2, delimitando el alcance del problema de acuerdo con la disponibilidad de datos para analizarlo. En la delimitación se determinó que el área de actuación se acotó a una red de aprovisionamiento, cuyos requerimientos se centran en la infraestructura de transporte de primera milla y terminales intermodales. A partir de estas necesidades se precisó el tipo de problema de decisión a un problema combinado LI-DR intermodal, así como los recursos disponibles para resolverlo y los criterios económicos, sociales y medioambientales más apropiados para optimizar las soluciones.

En el paso de construcción del modelo se requirió el modelado de los datos del problema y de la estructura de la red de entrada, igualmente, se tomó como punto de partida una formulación de referencia disponible en la literatura para efectuar la construcción gradual de la programación matemática que permitió proponer 2 versiones representadas en 5 variantes. Con el modelado de los datos del problema y de la estructura de la red de entrada se establecieron elementos de base para la representación matemática del problema de investigación, detallándose las variables de decisión de nivel estratégico y táctico, y la direccionalidad y tipo de asignación de los flujos en los nodos y arcos de las redes subyacentes. La formulación propuesta por Amaral et al. (2012) se utiliza como referencia para la construcción gradual de las formulaciones (puntualmente para las variantes mono-periodo). 
Finalmente, para llevar a cabo el análisis de decisiones se implementaron dos esquemas de resolución. Los esquemas de resolución se implementaron en R + Gurobi y Python + Gurobi, utilizando la estructura de grafo de la red de entrada para indexar las decisiones del problema combinado LI-DR intermodal. Con el primer esquema se aplicaron pautas de indexación con rutinas para generar matrices, que permitieron construir la matriz A para resolver los MPLEM mono-periodo. Con el segundo esquema se programaron secuencias de instrucciones basadas en iteraciones y condicionales, el cual se aplicó para todas las variantes multi-periodo. Para resolver los modelos se generaron instancias que proporcionaron resultados satisfactorios para analizar el comportamiento de las soluciones. 


\section{CAPÍtTULO 4}

Este capítulo se redactó a partir de una síntesis de los artículos referidos a continuación, y se complementó con nueva literatura. Los artículos son:

Intermodal transport in freight distribution: a literature review. Agámez-Arias, A. \& Moyano-Fuentes, J. Publicado en la revista Transport Reviews, 37(6), 782-807, 2017. https://doi.org/10.1080/01441647.2017.1297868

A systematic literature review of the design of intermodal freight transportation networks addressing location-allocation decisions. Agámez-Arias, A. García-Sabater, J. Ruiz, A. \& Moyano-Fuentes, J. Publicado en la revista European Journal of Industrial Engineering, 15(1), 1-34, 2021. https://doi.org/ 10.1504/EJIE.2021.113506 


\title{
Capítulo 4
}

\section{Intermodalidad y problemas combinados de localización de instalaciones-diseño de red: estado del arte}

\begin{abstract}
Resumen: en este capítulo se presentan los resultados del análisis de la literatura sobre los problemas combinado de localización de instalaciones-diseño de red (LI-DR) intermodal. La revisión se llevó a cabo desde dos perspectivas. En la primera se identifican los factores clave en la composición y funcionamiento de los sistemas intermodales y se caracterizan los problemas de decisión, aplicando los pasos propuestos por Denyer \& Tranfield (2009) para una revisión sistemática de literatura (RSL). En la segunda se realiza una propuesta novedosa que vincula tres nuevos pasos como etapa previa a la RSL, denominándola revisión sistemática de la literatura en dos etapas vinculadas (RSL-2EV). Con la RSL-2EV se analizan las contribuciones de los estudios centrados en los problemas combinados LI-DR que utilizan la programación matemática como técnica de resolución. Tanto para la RSL como para la RSL2EV se proponen clasificaciones novedosas que facilitan la interpretación de la literatura y actualización futura de la misma. En el presente capítulo se sintetizan los principales hallazgos, destacando especialmente los resultados obtenidos para la segunda perspectiva.
\end{abstract}




\subsection{Introducción}

El modelado explícito de la intermodalidad en los problemas de decisión en el campo del transporte comenzó a formalizarse en la década de 2000. Como consecuencia, los límites de la investigación en este campo de estudio se han extendido progresivamente, especialmente, en lo referido al transporte de mercancías (Agámez-Arias et al., 2021). Las primeras aportaciones de la literatura destacan tópicos relacionados con el transporte ferroviario, los operadores de transporte de corta distancia, métodos de transbordo de la carga (Bontekoning et al., 2004) y el desarrollo de técnicas de programación matemática (Macharis \& Bontekoning, 2004). Desde entonces las líneas de investigación se diversificaron, centrándose en la resolución de problemas de decisión para la localización de instalaciones, diseño de la terminal, asignación de la demanda, servicios en la terminal, enrutamiento de vehículos, ordenes de carga (Caris et al., 2008; SteadieSeifi et al., 2014), desarrollo de políticas públicas (Pallme at al., 2015) y triple enfoque de sostenibilidad (Zhao et al., 2020), entre otros.

Los problemas mencionados se han analizado y clasificado de acuerdo con la propuesta clásica de los tres niveles de planificación: estratégico, táctico y operativo (Caris et al., 2008; Crainic, 2000; Elbert et al., 2020; Macharis \& Bontekoning, 2004; SteadieSeifi et al., 2014). Sin embargo, existen problemas que combinan decisiones de los distintos niveles de planificación, por ejemplo, combinan la localización de instalaciones con la asignación de la demanda (Farahani \& Hekmatfar, 2009), con decisiones de rutas (Schneider \& Drexl, 2017), con decisiones de inventario (Davidson \& Leachman, 2012) o con el diseño de la red (Ghaderi \& Jabalameli, 2013). Para estos casos, la propuesta clásica de clasificación no permite analizar adecuadamente la literatura existente. Por lo tanto, es necesario llevar a cabo una revisión de la literatura basado en una clasificación novedosa, que aporte conocimiento diferenciado respecto a los principales aspectos abordados en los problemas que combinan las decisiones de planificación.

En esta tesis se estudia un problema combinado de localización de instalaciones-diseño de red (LI-DR) intermodal. Este tipo de problema integra la localización de instalaciones con el diseño de la red a partir de la estructura de las redes de transporte subyacentes (OrtizAstorquiza et al., 2018). Estos, básicamente pretende decidir, a nivel estratégico, cuáles son los nodos y arcos a localizar que diseñan la red, y a nivel táctico, cómo se conectan los nodos y se asigna la demanda (Sanci \& Daskin, 2019). Debido a la cantidad de literatura disponible con implicación del transporte intermodal en la toma de decisiones, tanto como el elevado número de aspectos que pueden confluir en los problemas combinados LI-DR y el enfoque metodológico empleado para resolverlos, se llevan a cabo dos revisiones de literatura. En la primera se identifican las aportaciones en torno a los factores clave en la composición y funcionamiento de los sistemas de transporte intermodal, y en la segunda, se analizan los problemas combinados LI-DR intermodal. En ambas se aplican los pasos propuestos por Denyer \& Tranfield (2009) para una revisión sistemática de literatura (RSL). En la segunda, además, se realiza una propuesta novedosa que vincula tres nuevos pasos como etapa previa a la RSL, denominándola revisión sistemática de la literatura en dos etapas vinculadas (RSL2EV). 
En el presente capítulo se sintetizan los principales hallazgos obtenidos de las dos revisiones $^{29}$, y se presentan dando cuenta de los factores clave para el funcionamiento de los sistemas de transporte intermodal, el alcance de los problemas combinados LI-DR intermodal, y el estado actual de la literatura referida a este tipo de problemas combinados. En la sección 4.2. se introduce la conceptualización del transporte intermodal y se profundiza en la eficiencia económica y la calidad del servicio como factores clave del sistema. En la sección 4.3. se identifican los problemas de decisión clasificados en la literatura y se puntualiza en los problemas de localización de instalaciones, problemas de diseño de red y los problemas combinados LI-DR. En la sección 4.4. se presenta el estado actual de la investigación de los problemas combinados LI-DR intermodal que utilizan la programación matemática como técnica de análisis de decisiones. En la sección 4.5. se presenta la discusión y se proponen las líneas de investigación con potencial de desarrollo. Finalmente, en la sección 4.6. se detallan las conclusiones de este capítulo.

\subsection{La intermodalidad en el transporte}

El concepto de transporte intermodal se refiere al uso de dos o más modos de transporte en el movimiento, en una unidad de carga, de mercancías o personas desde el origen hasta el destino (Crainic \& Kim, 2007). En la esencia de esta definición se distinguen otros términos que, desde la operativa del transporte, producen interesantes discrepancias entre ellos. Los términos son: transporte multimodal, transporte combinado, transporte co-modal y transporte sincromodal. Agámez-Arias \& Moyano-Fuentes (2017), Reis et al. (2013), SteadieSeifi et al. (2014) y Verweij (2011) analizan estas diferencias, las cuales se detallan en la Tabla 4.1. Por ejemplo, la unidad de carga, la manipulación de la mercancía, el modo de transporte utilizado, el uso eficiente de los recursos disponibles en cada modo de transporte y la sincronización del sistema de transporte, son algunos aspectos que establecen las diferencias entre los términos.

Con excepción del transporte sincromodal, la literatura no suele extenderse en las precisiones conceptuales para ofrecer alternativas de solución a los problemas de decisión estudiados. El transporte intermodal y multimodal son términos que se emplean como sinónimos tanto en la industria como entre investigadores y académicos (Dua \& Sinha, 2019). El transporte combinado se utiliza con énfasis en los documentos que promulgan las normativas de ordenación del transporte y circulación de mercancías entre los estados miembros de la Unión Europea (UNECE, 2001). La literatura sobre transporte co-modal ha tenido un desarrollo limitado, entre las escasas aportaciones sobresalen soluciones para el transporte de pasajeros (Dotoli et al., 2017). Por su parte, el transporte sincromodal ha captado rápidamente la atención debido al profundo impacto que ejercen las decisiones de elección de modo y uso de la infraestructura frente a problemáticas que estudian los retrasos, perturbaciones, congestión, confiabilidad y preocupaciones ambientales (Ambra et al., 2019).

\footnotetext{
${ }^{29}$ En ambas revisiones los pasos se cumplimentaron de manera exhaustiva para proponer clasificaciones novedosas e identificar los vacíos en la literatura. No obstante, para conservar la cohesión a lo largo del capítulo y la coherencia y proporcionalidad del capítulo en la unidad del documento, se hace mayor hincapié en los resultados de la RSL-2EV. En el anexo D se presenta un consolidado del proceso de revisión llevado a cabo en la RSL, mientras lo correspondiente a la RSL2EV se expone directamente en la subsección 4.4. Para consultar de manera detallada todos los resultados se recomienda acceder directamente a los artículos, cuyos datos se encuentran en la primera página de este capítulo y en el correspondiente apartado de referencias: Agámez-Arias \& Moyano-Fuentes (2017) y Agámez-Arias et al. (2021).
} 
Aunque en el desarrollo de este documento nos referimos explícitamente al término transporte intermodal, la literatura referenciada en toda su extensión se consultó considerando todos los términos presentados en la Tabla 4.1. Respecto a la literatura sobre transporte sincromodal, se tuvo en cuenta que los aportes correspondieran con el problema de investigación que se estudia en esta tesis.

Tabla 4.1. Transporte intermodal y términos relacionados

\begin{tabular}{|c|c|}
\hline Término & Definición \\
\hline \multirow{3}{*}{$\begin{array}{l}\text { Transporte } \\
\text { intermodal }\end{array}$} & Se emplea con mayor frecuencia en el contexto del transporte en EEUU. \\
\hline & $\begin{array}{l}\text { Se define una unidad de carga para efectuar el movimiento entre los modos de transporte, } \\
\text { siendo el contenedor la unidad de carga más utilizada. }\end{array}$ \\
\hline & Se concentra en efectuar el movimiento de la mercancía y no su manipulación. \\
\hline \multirow{3}{*}{$\begin{array}{l}\text { Transporte } \\
\text { multimodal }\end{array}$} & Se emplea con mayor frecuencia en el transporte de carga transoceánica. \\
\hline & Se puede definir, o no, una unidad de carga para mover la mercancía. \\
\hline & $\begin{array}{l}\text { Generalmente se establece un contrato entre el expedidor y el operador de transporte } \\
\text { multimodal para entregas que sobrepasan una frontera territorial (exportaciones). }\end{array}$ \\
\hline \multirow{3}{*}{$\begin{array}{l}\text { Transporte } \\
\text { combinado }\end{array}$} & Se emplea con mayor frecuencia en el contexto del transporte en Europa. \\
\hline & $\begin{array}{l}\text { El trayecto más extenso utiliza principalmente el modo ferroviario, y de acuerdo con el } \\
\text { área geográfica los modos fluvial y marítimo. El modo por carretera implica un mínimo } \\
\text { grado de uso, destacando solo en los tramos iniciales y finales. }\end{array}$ \\
\hline & Se debe constar la documentación correspondiente para el uso de cada modo. \\
\hline $\begin{array}{l}\text { Transporte } \\
\text { co-modal }\end{array}$ & $\begin{array}{l}\text { Se centra en el uso eficiente de los recursos de uno o más modos de transporte en un } \\
\text { sistema de distribución con uno o varios remitentes, para obtener los mayores beneficios } \\
\text { referidos a la sostenibilidad, seguridad y flexibilidad en la distribución. }\end{array}$ \\
\hline \multirow[t]{2}{*}{$\begin{array}{l}\text { Transporte } \\
\text { sincromodal }\end{array}$} & $\begin{array}{l}\text { Se requiere la coordinación y sincronización entre los actores de la red de transporte y de } \\
\text { los servicios logísticos para optimizar en tiempo real el uso de los sistemas de transporte. }\end{array}$ \\
\hline & La elección del modo y la ruta no están predefinidas con antelación. \\
\hline
\end{tabular}

Fuente: Adaptado de Agámez-Arias \& Moyano-Fuentes (2017)

En todo sistema de transporte de mercancías se debe alcanzar altos niveles de desempeño tanto en términos de eficiencia económica como de calidad del servicio. Los costes, la competencia en el mercado y los estándares de servicio en relación con los tiempos y la confiabilidad son factores de decisión fundamentales para el buen funcionamiento del sistema (Crainic \& Laporte, 1997). Se ha demostrado que la contratación de un operador de transporte intermodal sería hasta un $80 \%$ más barato que contratar una solución solo por carretera (Pinto et al., 2018) y que, además, la opción intermodal puede reducir significativamente las emisiones de $\mathrm{CO}^{2}$ (Ayar \& Yaman, 2012). A continuación, se profundiza en los factores clave de los sistemas de transporte intermodal. En la subsección 4.2.1. se realiza una descripción de los aspectos que inciden en la eficiencia económica de los sistemas, y en la subsección 4.2.2. aquellos que impactan en la calidad del servicio. 


\subsubsection{Eficiencia económica del sistema}

Los sistemas de transporte son sistemas complejos que involucran una gran cantidad de recursos humanos y materiales y muestran intrincadas compensaciones entre los componentes del sistema (Crainic \& Laporte, 1997). Las decisiones de planificación tienen una relación directa con la eficiencia del sistema. A medida que se potencia el uso de los recursos modales y se minimizan los movimientos de transporte, se logran mejoras significativas en los costes de transporte y operativos de transbordo en las terminales intermodales (Corry \& Kozan, 2008). La gestión conjunta del transporte y los inventarios producen un cambio de paradigma en la visión tradicional del almacenamiento. Las velocidades y tiempos en la actividad de transporte permiten incorporar el concepto de almacenamiento en tránsito, transformando la estructura de la oferta de los servicios logísticos y ocasionando con ello la reducción de los costes y tiempos de almacenamiento (Dekker et al., 2009).

Las terminales intermodales (Cho et al., 2012), el uso máximo de la capacidad (Pinto et al., 2018), los fletes según el modo de transporte (Ayar \& Yaman, 2012) y los sistemas de información para la trazabilidad y control de la carga y las actividades logísticas son de vital importancia en la planificación de estos sistemas (Sternberg et al., 2010). Igualmente, la sinergia entre los actores y agentes facilitan la integración en la oferta de servicios y mejora el rendimiento global del sistema dando lugar al uso especializado de los modos de transporte (Pallme et al., 2015; Reis et al., 2013). Las operaciones intermodales en comparación con las operaciones por carretera mitigan el cambio climático, por ejemplo, en un sistema carreteraferroviario se puede lograr una reducción de las emisiones hasta en $77 \%$ y mejorar en $43 \%$ el consumo de combustibles (Pinto et al., 2018). Sin embargo, la intensificación del comercio mundial genera fuertes presiones sobre los actores de las cadenas de suministro, la cadena de transporte y gobiernos, para continuar implementando soluciones que atenúen los efectos negativos de la actividad del transporte en el medio ambiente y la sociedad (Ambrosino \& Sciomachen, 2021).

Las principales soluciones intermodales se concentran en el desarrollo de la red de instalaciones y de infraestructura de transporte modal coherentes con las estrategias de crecimiento sostenible del país o de un contexto geográfico particular. Por ejemplo, en Alemania se ha impulsado el desarrollo de terminales portuarias interiores y el transporte fluvial (Biermann et al., 2015), en Italia se ha reconocido la falta de alineación entre las estrategias de orden nacional y regional para impulsar el uso de los puertos y las terminales intermodales existentes como infraestructuras integradas en un mismo sistema (Monios, 2016), y en Croacia se han implementado programas de inversión en nueva infraestructura logística intermodal (Bendekovic et al., 2014). En general, en la Unión Europea la intermodalidad ha resultado en reducción de los costes logísticos, mayor accesibilidad a los mercados e incremento de la competitividad regional (Darabann et al., 2012). Para aprovechar el efecto desbordamiento que supone la implementación de estas iniciativas para otras regiones, Rumanía procura acoplar la inversión en nueva infraestructura con las redes de transporte europeas (Tudorica \& Banacu, 2017).

Por el contrario, en EE. UU. pese a que se han promulgado leyes como la Ley de eficiencia del transporte intermodal (ISTEA '91) y la Ley de equidad en el transporte para el siglo XXI (TEA-21), más del 60\% de la carga sigue moviéndose por las carreteras debido a que el sistema intermodal ocasiona altos costes que hacen poco viable el uso de algunas terminales (Badyal 
et al., 2020). En los países de Latinoamérica como México, la fragmentación de los sistemas modales y el desarrollo desproporcionado de la infraestructura a nivel interno impiden el acceso a los puertos marítimos, fluviales y secos, y a las terminales ferroviarias, por ende, el desarrollo de la intermodalidad se ve rezagado (Wilmsmeier et al., 2015). En Brasil se analizan soluciones intermodales atendiendo a la situación de transporte de cada subregión con la finalidad de implementar corredores intermodales con terminales de distintas características para reducir costes y tiempos de servicio (Isler et al., 2020). Mientras que en Chile se aprovecha la heterogeneidad de los sistemas modales de transporte para promover la optimización en el uso de los vehículos marítimos para el desarrollo de sistemas intermodales sostenibles (MartínezLópez \& Chica, 2020).

\subsubsection{Calidad del servicio}

La adecuada trazabilidad en la oferta de servicios de transporte intermodal permite mejorar la forma en que se ejecutan las actividades logísticas y, por ende, la calidad del servicio. No obstante, los aspectos más importantes para la calidad del servicio difieren de cada actor del sistema (Kreutzberger, 2008). El consignatario la percibe de acuerdo a la relación serviciocoste, por lo que la planificación estratégica de las instalaciones y el diseño de rutas es trascendental (Dua \& Sinha, 2019). El transportista considera aspectos relacionados con la gestión, almacenamiento, entrega de la carga y manejo de la información (Wisner, 1999). Los transportistas incentivan la colaboración entre las empresas para planificar las actividades (Crainic \& Kim, 2007; Puettmann \& Stadtler, 2010) y así favorecer la disponibilidad, frecuencia y flexibilidad de los servicios (Saeed, 2013). Bontekoning et al. (2004) afirman que, entre consignatarios y transportistas, las compensaciones desproporcionadas de los costes y los tiempos afectan la calidad del servicio.

Para el cliente, la puntualidad en la entrega de la carga, la comunicación constante y los precios competitivos son aspectos importantes (Evers \& Johnson, 2000). Por ello, es indispensable determinar la capacidad general de los proveedores del sistema, y la capacidad particular de los operadores de las terminales para efectuar rápidamente el transbordo de los contenedores (Stank \& Roath, 1998; Van Zyl, 1992). Los métodos como Justo a Tiempo exigen la ejecución adecuada del transbordo y debido a la poca manipulación de la mercancía los resultados permiten una mayor seguridad y limpieza de la misma (Wieslaw et al., 2016). Sin embargo, estos métodos requieren de un sistema de apoyo basado en nuevas tecnologías, accesibilidad a los datos, herramientas analíticas (Dua \& Sinha, 2019) y profesionales especializados para prestar un servicio eficiente (Leviakangas et al., 2007; Sternberg et al., 2010).

El intercambio de datos entre los actores es complejo, los sistemas de información pueden ser incompatibles o por falta de confianza no se revela información de las empresas involucradas (Braekers et al., 2013). Los proveedores de soluciones tecnológicas juegan un papel estratégico al promover una mayor adopción de las tecnologías para mejorar la calidad del servicio (Harris et al., 2015). Algunas de estas facilitan el intercambio de información en tiempo real para triangular los contenedores y reducir los movimientos en la terminal (Kelleher et al., 2003), mejorar la seguridad de la mercancía durante el transporte (Lun et al., 2008) o comparar el sistema intermodal con los sistemas unimodales considerando las interrupciones y la confiabilidad para la selección de las rutas (Dewan et al., 2013). Asimismo, los sistemas 
electrónicos permiten gestionar los trámites administrativos y aduaneros requeridos, a la vez que contribuyen a que el transporte sea más seguro, efectivo y eficiente (Giannopoulos, 2004).

Por otra parte, los sistemas de transporte inteligentes mejoran los niveles de visibilidad y capacidad de respuesta de los sistemas intermodales (Coronado-Mondragon et al., 2012). Aquellos que incorporan el enrutamiento, programación, monitoreo y rastreo de mercancías permiten optimizar las decisiones en las rutas (Gilmore \& Tompkins, 2000). Actualmente, estos sistemas de apoyo son imprescindibles para la efectividad y eficiencia del servicio (Marchet et al., 2012). Además, se requiere la integración de los sistemas para supervisar y planificar las distintas operaciones a lo largo y ancho de la red (Casu et al., 2013). Para esta integración es fundamental la cooperación de todos los actores y la medición del desempeño (Dua \& Sinha, 2019). La débil o nula integración de los actores del sistema intermodal imposibilitan el manejo adecuado de la carga y la información (Almotairi et al., 2011).

\subsection{El transporte intermodal y la planificación de las decisiones}

Caris et al. (2008), conservando la estructura clásica de planificación de decisiones estratégico, táctico y operativo, clasifican la literatura sobre el transporte intermodal. La Tabla 4.2. muestra una estructura general de los problemas de decisión identificados para cada nivel de planificación y actor de la red intermodal. De todos ellos, la localización de terminales ha tenido una extensa atención en la literatura, con aplicaciones cada vez más atractivas (Farahani et al., 2010). La planificación táctica en el transporte intermodal se ha simplificado a decisiones de asignación con valores de costes que en su mayoría ignoran los problemas de gestión de vehículos y la configuración de las redes subyacentes (Elbert et al., 2020). El nivel operacional, en comparación con los otros dos niveles, ha sido menos analizado (Ahumada \& Villalobos, 2009) estando algunas propuestas relacionadas a problemas dinámicos que incorporan periodos tácticos/operacionales en los periodos estratégicos (Govindan et al., 2017).

Tabla 4.2. Clasificación de los problemas de decisión por nivel de planificación y actor de la red intermodal

\begin{tabular}{llll}
\hline Término & Nivel estratégico & Nivel táctico & Nivel operacional \\
\hline $\begin{array}{l}\text { Operador de } \\
\text { transporte de } \\
\text { corta } \\
\text { distancia }\end{array}$ & $\begin{array}{l}\text { Cooperación entre } \\
\text { empresas de transporte } \\
\text { Tamaño de la flota de } \\
\text { camiones y chasis }\end{array}$ & $\begin{array}{l}\text { Asignación de cargadores } \\
\text { y del receptor de una } \\
\text { terminal }\end{array}$ & $\begin{array}{l}\text { Ruta de vehículos } \\
\text { Redistribución del } \\
\text { remolque y unidades de } \\
\text { carga }\end{array}$ \\
\hline $\begin{array}{l}\text { Operador de } \\
\text { la terminal }\end{array}$ & $\begin{array}{l}\text { Diseño de la terminal: } \\
\text { atracaderos, }\end{array}$ & $\begin{array}{l}\text { Niveles de capacidad de } \\
\text { equipos y mano de obra }\end{array}$ & $\begin{array}{l}\text { Gestión de recursos } \\
\text { Programación de trabajos }\end{array}$ \\
& almacenamiento & $\begin{array}{l}\text { Rediseño de rutinas } \\
\text { operativas y estructuras de } \\
\text { diseño }\end{array}$ & \\
\hline $\begin{array}{l}\text { Operador de } \\
\text { la red }\end{array}$ & $\begin{array}{l}\text { Configuración de la red de } \\
\text { infraestructura }\end{array}$ & $\begin{array}{l}\text { Configuración red de } \\
\text { consolidación }\end{array}$ & $\begin{array}{l}\text { Orden de carga de trenes } \\
\text { Redistribución de vagones, } \\
\text { barcazas y unidades de } \\
\text { carga }\end{array}$ \\
\hline & $\begin{array}{l}\text { Localización de la } \\
\text { terminal/hub }\end{array}$ & Modelo de producción & \\
\hline
\end{tabular}




\begin{tabular}{llll}
\hline & & Estrategias de precios & $\begin{array}{l}\text { Replanificación de } \\
\text { itinerario }\end{array}$ \\
\hline $\begin{array}{l}\text { Operador de } \\
\text { transporte } \\
\text { intermodal }\end{array}$ & $\begin{array}{l}\text { Cooperación entre } \\
\text { empresas de transporte }\end{array}$ & $\begin{array}{l}\text { Asignación única y } \\
\text { múltiple a las } \\
\text { terminales/hubs }\end{array}$ & $\begin{array}{l}\text { Enrutamiento y } \\
\text { reposicionamiento de la } \\
\text { unidad de carga }\end{array}$ \\
& & $\begin{array}{l}\text { Elección de los servicios y } \\
\text { modos de transporte }\end{array}$ & \\
\hline
\end{tabular}

Fuente: Adaptado de Caris et al. (2008)

SteadieSeifi et al. (2014) ofrecen una revisión actualizada de la literatura manteniendo la estructura propuesta por Caris et al. (2008). Crainic \& Kim (2007) exponen que, en la adaptación de los problemas del mundo real como modelos de programación matemática, algunas decisiones y criterios no se plantean de manera explícita en la formulación, es por ello que los problemas estudiados pueden estar clasificados en uno de los tres niveles de planificación. Por lo tanto, las contribuciones de los dos problemas que se combinan en los LIDR se encuentran mayoritariamente por separado (Rahmaniani \& Ghaderi, 2013). El análisis individualizado ha conllevado a proponer otras clasificaciones que se centran en distinguir como se han estudiado aspectos que caracterizan la programación matemática. La Figura 4.1. resume los más genéricas. La diferenciación entre las posibilidades de cada aspecto se esclarece en las siguientes subsecciones. La subsección 4.3.1. trata los problemas de localización de instalaciones y la subsección 4.3.2. los problemas de diseño de red. Luego, en la subsección 4.3.3. se realiza una descripción de los problemas combinados LI-DR y se introducen algunos antecedentes.

Figura 4.1. Aspectos genéricos de los modelos de programación matemática

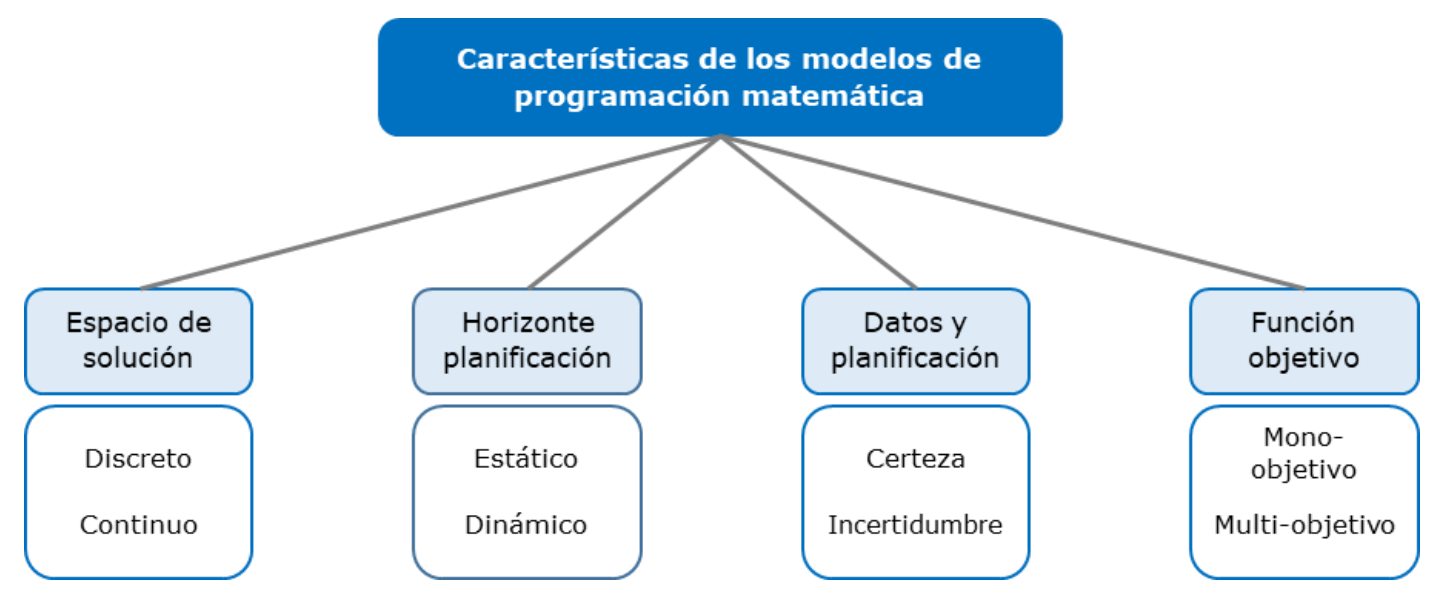

Fuente: Elaboración propia a partir de Arabani \& Farahani (2012), Farahani, Hekmatfar, et al. (2013) y Mansouri et al. (2015)

\subsubsection{Localización de instalaciones}

Los problemas de localización de instalaciones son problemas de nivel estratégico. Estos contemplan la búsqueda de la localización de una instalación que mejor satisface la demanda de uno o varios puntos, por lo que usualmente se minimizan los costes totales, tiempos y 
distancias de viaje (Contreras \& Fernández, 2012), o también se maximiza el nivel de servicio y los beneficios (Ansari et al., 2018).

El número de instalaciones a localizar puede ser única o múltiple y ser definida de manera exógena o endógena, este último en el proceso de optimización. Si se permite la localización de las instalaciones en cualquier punto del espacio de soluciones se hace referencia a un problema continuo, pero si se proporcionan los sitios candidatos de la localización es un problema discreto (Alizadeh et al., 2015). La consideración del tiempo conduce a diferenciar los problemas estáticos de los dinámicos; en los dinámicos se decide dónde y cuándo disponer de las instalaciones para satisfacer la demanda (Arabani \& Farahani, 2012). En aquellos problemas cuyas condiciones futuras son inciertas, se desconocen algunos datos del problema o estos son imprecisos, se hace referencia a modelos basados en la incertidumbre (Chen et al., 2011; Govindan et al., 2017). En la vida real las decisiones de planificación en los sistemas de transporte intermodal son tomadas por varios decisores, es por ello que para la localización de las instalaciones pueden surgir múltiples objetivos que impliquen algún grado de compromiso o conflicto entre los mismos (Baykasoğlu et al., 2019).

Los problemas con característica discreta constituyen un grupo importante y de interés para los investigadores, tanto por su aplicabilidad a problemas de la vida real, ya sea en la localización de un puerto o centros de distribución, como por el desarrollo de los procedimientos de resolución (Farahani, Hekmatfar, et al., 2013). Los problemas con característica dinámica han proporcionado una ventana de posibilidades en el análisis de la toma de decisiones de las instalaciones en función de los cambios en el tiempo, bien sea con ajustes a la capacidad de las instalaciones o modificando la permanencia de las instalaciones (Jena et al., 2015). Los ajustes en la capacidad refieren a la expansión, reducción o transferencia de ésta (Melo et al., 2009). Las modificaciones en la permanencia consisten en el cierre, reapertura o relocalización de la instalación, ya sea de manera definitiva o en un periodo determinado del horizonte de planificación (Arabani \& Farahani, 2012). Algunos estudios se relacionan con la expansión de la red de instalaciones (Fotuhi \& Huynh, 2018), los plazos de entrega (Correia \& Melo, 2016), las interrupciones y la sostenibilidad (Maiyar \& Thakkar, 2019).

La profunda evolución de los problemas de localización de instalaciones, tanto en la cadena de suministro como en la cadena de transporte, permiten identificar subdivisiones o agrupaciones centradas en otras decisiones y aspectos estipulados en los modelos propuestos. Por semejanza con el problema que se estudia en esta tesis, los de mayor interés son los que incluyen decisiones de asignación. Entre estos, los problemas de localización-asignación de instalaciones y los problemas de localización de instalaciones en la red. En un problema de localización-asignación de instalaciones se pretende encontrar las localizaciones para un conjunto de instalaciones que mejor sirvan a un conjunto de puntos de demanda y, al mismo tiempo, encontrar la asignación de cada punto de demanda a la instalación que mejor le sirva (Alumur et al., 2012). En tales modelos, la asignación puede ser única o múltiple, con el objetivo de minimizar los costes de asignación o apertura de la instalación (Fathollahi-Fard \& Hajaghaei-Keshteli, 2018). Estos varían en complejidad, encontrando desde modelos capacitados o no capacitados, estáticos, dinámicos (Klose \& Drexl, 2005), multi-objetivos, multi-objetivos multi-criterio (Farahani et al., 2010), estocásticos y difusos (Alizadeh et al., 2015). 
En los problemas de localización de instalaciones en la red las decisiones se configuran en términos de una red, es decir nodos y arcos, y las demandas pueden ocurrir en ambos de manera simultánea. Las instalaciones sirven como puntos de consolidación, clasificación y transbordo para aprovechar las economías de escala (Alumur \& Kara, 2008). Las decisiones de asignación, única o múltiple, se definen entre los puntos de demandas y las instalaciones, así como entre las instalaciones (Ishfaq \& Sox, 2012). En algunas ocasiones se incorporación las redes de transporte subyacentes para determinar la asignación (Papadimitriou et al., 2018). Entre estos se encuentran los problemas de mediana, problemas de centro, problemas de cobertura, problemas de ubicación central y problemas de ubicación jerárquica ${ }^{30}$. Recientemente, se ha estudiado los efectos de la incertidumbre en el servicio de entrega (Mohammadi et al., 2019), los costes de transporte (Rahmati \& Neghabi, 2021), y los múltiples objetivos para reducir las emisiones contaminantes y los tiempos de entrega (Dukkanci et al., 2019). Sin embargo, el enfoque dinámico de estos problemas requiere más atención en la literatura (Badyal et al., 2020).

\subsubsection{Diseño de la red}

Los problemas de diseño de red son de nivel estratégico, representan una forma generalizada de los problemas de localización y se definen en términos de nodos y arcos (Crainic \& Laporte, 1997). Un nodo puede constituir un punto de origen de la carga, un punto de demanda, un punto de intercambio o la intersección de dos o más arcos. Los arcos representan las vías de una red de transporte y las decisiones se relacionan principalmente con la localización o activación de los arcos, la capacidad del arco, la selección de rutas o el flujo de productos (Alumur \& Kara, 2008). En estos problemas, los costes de localización del arco no dependen de los flujos que circulan por la red (Contreras \& Fernández, 2012). En la literatura los problemas de diseño de red se reconocen en tres formas: diseño de red discreta, diseño de red continua y diseño de red mixta. El diseño discreto trata de la adición de nuevos arcos en la red de transporte, el diseño continuo se ocupa de la expansión de la capacidad en los arcos existentes, y el diseño mixto involucra simultáneamente los arcos que deben mejorarse y los nuevos arcos que se agregan a la red o redes existentes (Hosseininasab \& Shetab-Boushehri, 2015).

Algunas aplicaciones se encuentran en los problemas de diseño de la red vial, diseño de la red de transporte público y en los problemas de localización-enrutamiento. El diseño de la red vial es un problema de toma de decisiones estratégicas con recursos financieros limitados, en el que se optimiza el desempeño de la red considerando explícitamente los flujos (Xu et al., 2016). Algunas decisiones refieren a la construcción de nuevos arcos, adición de carriles y arcos de doble sentido convertidos en arcos de un solo sentido (Jia et al., 2019). Inicialmente, los estudios minimizaban costes y tiempos de viaje, pero en investigaciones recientes se incluyen medidas sociales y ambientales como la accesibilidad espacial y las emisiones de gases contaminantes (Szeto et al., 2015). El diseño de la red de transporte público se relaciona con el diseño y programación de las rutas, considera tanto la topología de la red como la frecuencia y horarios de servicio para un único modo de transporte (Farahani, Miandoabchi, et al., 2013). Por último, en los problemas de localización-enrutamiento se deciden las

\footnotetext{
${ }^{30}$ Para profundizar en este grupo de problemas se pueden consultar las revisiones de literatura propuestas por Campbell \& O'Kelly (2012), Farahani, Hekmatfar, et al. (2013) y Ortiz-Astorquiza et al. (2018).
} 
instalaciones a localizar y las rutas de transporte que se deben definir para servir los puntos de demanda, utilizando un conjunto determinado de vehículos (Schneider \& Drexl, 2017).

\subsubsection{Combinación de los problemas de localización de instalaciones y el diseño de la red}

Los problemas combinados LI-DR implican decisiones estratégicas para localizar instalaciones y localizar arcos en las redes de transporte subyacente, y decisiones tácticas para asignar las instalaciones y cumplir con la demanda teniendo en cuenta las redes de transporte (Ortiz-Astorquiza et al., 2018). Estos problemas se han abordado en menor proporción en la literatura y han sido de relevante aplicación en las redes de transmisión de energía, telecomunicaciones, sistemas de transporte intermodal, sistemas de tuberías (Arnold et al., 2004), logística humanitaria (Sanci \& Daskin, 2019), la planificación regional (Brahami et al., 2020) y en los programas de reutilización de tierras (Contreras \& Fernández, 2012). Los problemas combinados LI-DR son complejos porque integran distintos niveles de decisión y grupos de interés (López-Ospina et al., 2021). Rahmaniani \& Ghaderi (2013) afirman que, en algunos problemas de la vida real suele ser más conveniente tomar decisiones sobre los arcos de las redes de transporte que solo localizar nuevas instalaciones. La incorporación de las redes de transporte subyacentes conducen a una extrema dificultad en la identificación de un único diseño óptimo de la red (Yoon \& Current, 2008).

En los problemas combinados LI-DR las decisiones se han modelado limitando la capacidad de las instalaciones (Melkote \& Daskin, 2001) y en los arcos (Jabalameli \& Mortezaei, 2011; Rahmaniani \& Ghaderi, 2013), definiendo una estructura jerárquica entre las instalaciones y para los flujos de múltiples productos (Yoon \& Current, 2008) estableciendo distintos tipos de instalaciones (Bigotte et al., 2010) y para la elección explicita del modo de transporte (Cordeau et al., 2006). Por su parte, la incertidumbre se ha contemplado en los costes de uso de la red (Shishebori et al., 2016), la demanda (Liu \& Kwon, 2020; Ukkusuri \& Patil, 2009) y en los tiempos de restauración de los arcos de la red (Sanci \& Daskin, 2019). La literatura aún es escasa sobre este problema y sobre la incorporación de las tres dimensiones de sostenibilidad. Una de estas propuestas es la de Kim et al. (2013), quienes evalúan la expansión de la capacidad y mejora de los tiempos y velocidad en la red, minimizando costes sociales de emisiones de GEI y costes de uso de la red con un presupuesto de inversión limitado. Brahami et al. (2020), por su parte, proponen un modelo de sostenibilidad económica y medioambiental para reducir los costes de inversión y operativos de toda la red y las emisiones de $\mathrm{CO}^{2}$ en los arcos que se localizan.

\subsection{Optimización de problemas combinados LI-DR intermodal: Una RSL-2EV}

Los problemas que combinan las decisiones de localización de las instalaciones y el diseño de la red con elección del modo de transporte son una línea de investigación prometedora, no solo desde el punto de vista de la técnica y el modelado sino también en términos de diseñar procedimientos de resolución eficientes (Contreras \& Fernández, 2012). Este tipo de problema combinado conduce a interesantes oportunidades para optimizar los sistemas de transporte intermodal (Caris et al., 2014). En esta sección se presenta el estado actual de la investigación de los problemas combinados LI-DR intermodal que utilizan la programación matemática como técnica de análisis, aplicando una RSL-2EV. La RSL-2EV responde a una propuesta 
novedosa de esta tesis, en la cual se vinculan tres nuevos pasos a los cincos establecidos en la RSL de Denyer \& Tranfield (2009), con la finalidad de alinear algunos aspectos indispensables en la revisión, clasificación y análisis de la literatura. En la subsección 4.4.1. se describen las etapas y pasos de la RSL-E2V. En las subsecciones 4.4.2. y 4.4.3. se presentan los resultados obtenidos, distinguiendo, respectivamente, los hallazgos para la cadena de transporte y la cadena de suministro.

\subsubsection{La RSL-2EV}

La RSL es una metodología idónea para conocer el estado actual de la investigación, su rigurosidad y reproducibilidad asegura la trazabilidad de los resultados (Denyer \& Tranfield, 2009). No obstante, es importante reconocer que gran parte de la literatura que trata del transporte intermodal, los problemas de localización de instalaciones, diseño de red y programación matemática han sido previamente analizados, y sus resultados se soportan en más de una docena de publicaciones. Con el propósito de prevenir posibles imprecisiones en el proceso de revisión y de reconocer las contribuciones de las revisiones de literatura publicadas, se lleva a cabo una RSL-2EV. La Figura 4.2. esquematiza el proceso de revisión aplicado en la RSL-2EV. En el apartado 4.4.1.1. se describen los pasos de la primera etapa, y en el apartado 4.4.1.2. los pasos de la segunda etapa.

Figura 4.2. Etapas y pasos de la RSL-2EV aplicada en los problemas combinados LI-DR

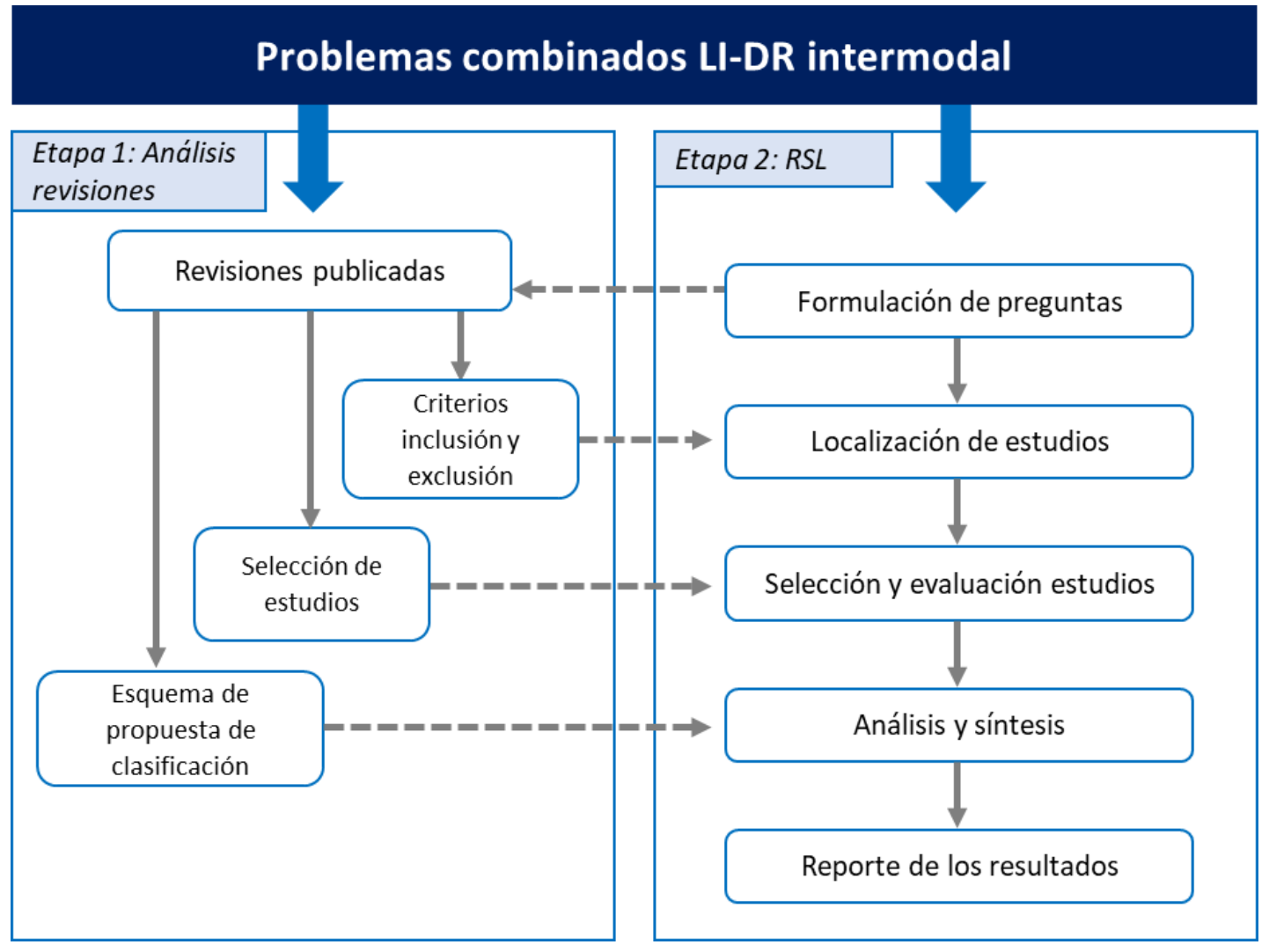

Fuente: Adaptado de Agámez-Arias et al. (2021) 


\subsubsection{Primera Etapa}

En esta etapa, a partir del análisis de las revisiones de literatura publicadas, se pretende afinar algunos supuestos para desarrollar, en la segunda etapa, los cinco pasos habituales de una RSL. Estos supuestos se enmarcan en tres aspectos: a) definición de criterios para rastrear los estudios, b) localización de estudios en las referencias citadas, y c) un esquema inicial de clasificación de la literatura. Las revisiones seleccionadas analizan modelos de programación matemática, principalmente, para problemas de planificación estratégica y/o estratégica-táctica en el ámbito de la cadena de transporte intermodal y la cadena de suministro intermodal. Agámez-Arias \& Moyano-Fuentes (2017), Baykasoğlu et al. (2019), Bontekoning et al. (2004), Bravo \& Vidal (2013), Caris et al. (2008), Caris et al. (2013), Caris et al. (2014), Contreras \& Fernández (2012), Dekker et al. (2012), Dua \& Sinha (2019), Farahani, Hekmatfar, et al. (2013), Khaslavskaya \& Roso (2020), Kumar \& Anbanandam (2019), Lam \& Gu (2013), Macharis \& Bontekoning (2004), Rožić et al. (2016) y SteadieSeifi et al. (2014) conforman este grupo de revisiones. En estas publicaciones se clasificaron aproximadamente 700 estudios y en conjunto abarcan el periodo 1981 a 2019.

Desde un contexto general del transporte intermodal, Bontekoning et al. (2004) proponen una agenda de investigación en los que destacan temas como el diseño de redes y la investigación de operaciones; Agámez-Arias \& Moyano-Fuentes (2017) identifican nuevas líneas de investigación relacionadas con los principios básicos, la eficiencia económica, y la modelización de los problemas del sistema. Centrados en las técnicas de investigación de operaciones, Macharis \& Bontekoning (2004), Caris et al. (2008) y SteadieSeifi et al. (2014) analizan y clasifican la literatura según el tomador de decisiones y el nivel de planificación del problema de investigación (estratégico, táctico y operativo) que se aborda en cada estudio. Baykasoğlu et al. (2019) se concentran en los problemas de planificación de flotas y Farahani, Hekmatfar, et al. (2013) en los problemas de localización de Hubs. Caris et al. (2013) exploran la preocupación ambiental y las tecnologías de la información enfatizando en la red de terminales y la red de servicios intermodales.

La creciente preocupación del impacto medioambiental y las decisiones de transporte en la gestión de la cadena de suministro han marcado una visión integradora que amplifica las propuestas de clasificación. Desde la actividad del transporte se puede afirmar que se han analizado los flujos logísticos en la cadena de suministro verde (Dekker et al., 2012), la calidad y el movimiento de mercancías en contenedores (Dua \& Sinha, 2019) o el diseño sostenible óptimo de la red de suministro incluyendo el "hinterland" portuario (Lam \& Gu, 2013). Caris et al. (2014) analizan la implicación del transporte por vías navegables. Khaslavskaya \& Roso (2020) y Rožić et al. (2016) se concentran en la participación de los puertos secos en las redes logísticas. Bravo \& Vidal (2013), Contreras \& Fernández (2012) y Kumar \& Anbanandam (2019) identifican los aspectos relevantes del modelado, las formulaciones y técnicas de resolución, en los cuales prima la elección del modo de transporte. Contreras \& Fernández (2012) abordan, de manera general, los problemas combinados LI-DR, y Kumar \& Anbanandam (2019) los problemas de diseño sostenible de la cadena de suministro intermodal.

El análisis de éstas revisiones permitió la identificación de los siguientes supuestos para llevar a cabo la segunda etapa de la RSL-2EV: 
- Con excepción de Contreras \& Fernández (2012), las revisiones citadas no analizan problemas combinados LI-DR. Si bien algunas revisiones o subsecciones de estas profundizan en la elección del modo de transporte y en las técnicas de programación matemática, se confirma que todavía hay un gran potencial para explorar la literatura entorno a este tipo de problema.

- En Caris et al. (2013) y Contreras \& Fernández (2012) se identifican estudios que pasan a conformar el grupo de preseleccionados, y en consecuencia con los periodos cubiertos por la literatura se delimita la localización de nuevos estudios a partir del año 2012.

- La diversidad de clasificaciones propuestas permite definir un listado de características claves utilizadas como punto de partida para catalogar la nueva literatura.

- Es interesante hacer seguimiento a conceptos emergentes relacionados con la sostenibilidad, el área geográfica y conectividad territorial, la toma de decisiones dinámica y la incertidumbre.

\subsubsection{Segunda Etapa}

En esta etapa se aplican los cinco pasos de la metodología de la RSL para conocer el estado actual de la investigación de los problemas combinados LI-DR intermodal aplicando programación matemática. Para la puesta en marcha de la RSL, en el primer paso, referido a los interrogantes de investigación, se formulan las siguientes preguntas para llevar a cabo la revisión:

- ¿Cuáles son las principales características de los problemas combinados LI-DR intermodal?

- ¿Qué aspectos se consideran en la toma de decisiones de este tipo de problema?

- ¿Qué técnicas de programación matemática se utilizan? y,

- ¿Qué temas emergentes se pueden identificar como futuras líneas de investigación?

En el segundo paso, para la localización de los estudios se definieron las bases de datos científicas, áreas de investigación, palabras claves y estrategias de búsqueda. La búsqueda se ejecutó en las bases de datos científicas WoS y Scopus. De acuerdo con los lineamientos identificados en la primera etapa, se limitó la búsqueda desde el 2012 y no se restringió el área de investigación. Esto permitió obtener resultados relacionados con las áreas de economía y negocios y planificación y desarrollo. Las principales palabras clave empleadas fueron: intermodal transport, intermodal freight transport, location-allocation problem, network design, intermodal network design y mathematical programming. La búsqueda se inició empleando operadores booleanos para combinar palabras clave y crear cadenas. Se truncó el término transport. De acuerdo con las apreciaciones de Agámez-Arias \& Moyano-Fuentes (2017) y SteadieSeifi et al. (2014) se implementaron los términos multimodal, synchromodal y co-modal como sinónimo de intermodal. Se aplicó un filtro para confirmar que los trabajos tuvieron un proceso de revisión por pares y se excluyeron estudios de los siguientes tipos: actas de conferencias, informes, libros, capítulos de libros y tesis/disertaciones. 
Para la selección y evaluación de los estudios en el tercer paso se consideraron diferentes filtros. Con el filtro inicial se determinó para cada estudio si existía correspondencia del título y el resumen con los interrogantes formulados en el primer paso de esta RSL, y en algunas ocasiones también se realizó la lectura de las conclusiones para descartar estudios dudosos. En aquellos que se confirmaba la correspondencia se agruparon como estudios preseleccionados, además, se adhirieron a este grupo los estudios identificados como resultados del desarrollo de la primera etapa. El siguiente filtro consistió en verificar entre los estudios preseleccionados si el problema de investigación conducía a la toma de decisiones estratégico-táctica y la propuesta de resolución a técnicas de programación matemática. También se utilizó la técnica de bola de nieve en las referencias citadas en los estudios preseleccionados ${ }^{31}$ para localizar más estudios. Finalmente, el grupo de seleccionados para ser evaluados se conforma por un total de 20 estudios $^{32}$.

Para llevar a cabo el análisis y síntesis en el cuarto paso, se identificaron 25 características clave categorizadas en cuatro ejes diferentes. El marco taxonómico propuesto por Bravo \& Vidal (2013) se tomó como referencia para definir las características que se presentan en la Tabla 4.3. En el eje 1, Particularidades del problema de investigación, se analiza en detalle la estructura de soporte de las redes. En el eje 2, Decisiones combinadas en el diseño intermodal de la red, se declara el conjunto de decisiones que integran la planificación estratégica y táctica de cada problema. El eje 3, Modelado de las decisiones de localización y asignación, se contempla la forma en cómo se adaptan las decisiones y los parámetros en la formulación matemática. El eje 4 se centra en la Estructura y resolución de la programación matemática.

El quinto paso de la RSL atañe al reporte de los resultados obtenidos del análisis de la literatura. Los resultados se presentan en la siguiente subsección.

Tabla 4.3. Características clave para analizar los estudios seleccionados en la RSL-2EV

\begin{tabular}{|c|c|c|c|}
\hline Eje & Características clave & Descripción & \\
\hline \multirow{7}{*}{ 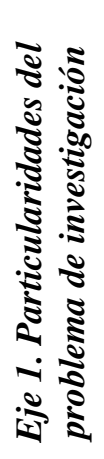 } & (A) Red de transporte & (A1) Cadena de transporte & (A2) Cadena de suministro \\
\hline & \multirow{5}{*}{ (B) Actores } & (B1) Operador de transporte & (B5) Proveedores \\
\hline & & \multirow{2}{*}{$\begin{array}{l}\text { (B2) Operador de la terminal } \\
\text { intermodal }\end{array}$} & (B6) Fabricantes \\
\hline & & & (B7) Centros de distribución \\
\hline & & $\begin{array}{l}\text { (B3) Operador de transporte de } \\
\text { corta distancia }\end{array}$ & (B8) Instalaciones logísticas \\
\hline & & (B4) Actores gubernamentales & (B9) Minoristas \\
\hline & (C) Actor por tipo & (C1) Único & (C2) Múltiple \\
\hline
\end{tabular}

\footnotetext{
${ }^{31}$ La técnica bola de nieve permitió ampliar la localización de estudios consultando la lista de referencias de los documentos preseleccionados. En las listas se identificaron aquellos estudios que, por el título, podían guardar relación con el objetivo de la revisión. Estos fueron rastreados, se aplicó el primer filtro y los elegibles pasaron a conformar el grupo de seleccionados.

${ }^{32}$ Dado que los problemas combinados LI-DR en el campo del transporte se han estudiado en menor proporción en la literatura y las particularidades del problema de investigación de esta tesis acotan los criterios de elegibilidad de los estudios, el grupo de los seleccionados se podría percibir reducido. Sin embargo, estos representan el $41 \%$ del total de estudios que conforman el grupo de los preseleccionados (estos se listan en el anexo E).
} 


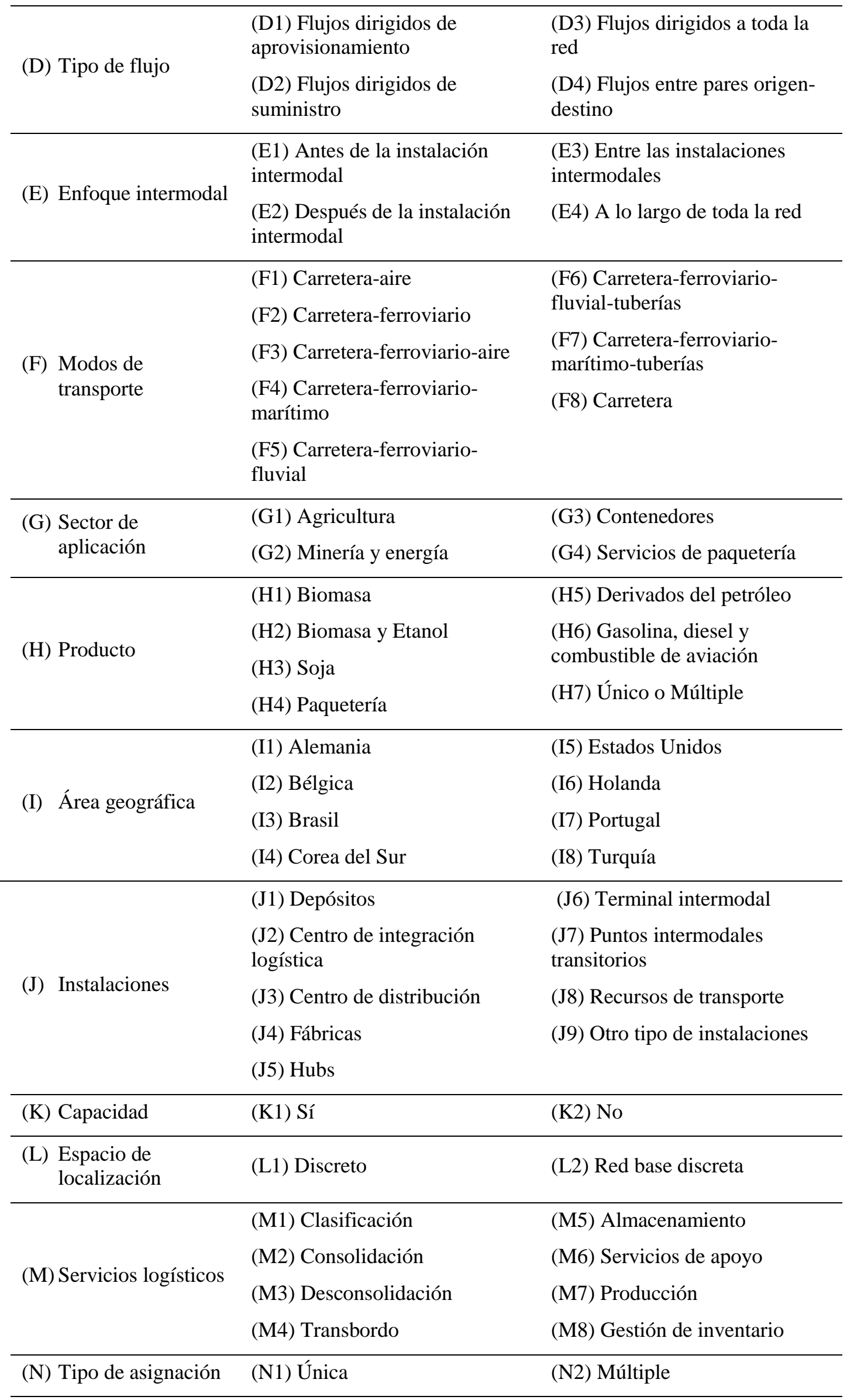




\begin{tabular}{|c|c|c|c|}
\hline & $\begin{array}{l}\text { (O) Atributos de } \\
\text { asignación }\end{array}$ & $\begin{array}{l}\text { (O1) Costes de transbordo y de } \\
\text { transporte } \\
\text { (O2) Distancias } \\
\text { (O3) Velocidades } \\
\text { (O4) Tiempos }\end{array}$ & $\begin{array}{l}\text { (O5) Capacidad del vehículo o } \\
\text { unidad de carga } \\
\text { (O6) Inventarios } \\
\text { (O7) Emisiones GEI } \\
\text { (O8) Capacidad del arco }\end{array}$ \\
\hline \multirow{14}{*}{ 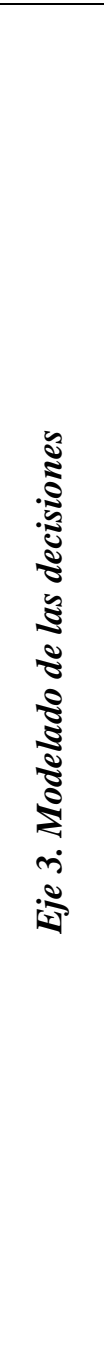 } & \multirow{5}{*}{ (P) Decisiones de red } & (P1) Ubicación de la instalación & (P6) Asignación \\
\hline & & $\begin{array}{l}\text { (P2) Ubicación de la instalación } \\
\text { con capacidad }\end{array}$ & $\begin{array}{l}\text { (P7) Asignación entre } \\
\text { instalaciones }\end{array}$ \\
\hline & & $\begin{array}{l}\text { (P3) Ubicación con operaciones } \\
\text { en la instalación }\end{array}$ & $\begin{array}{l}\text { (P8) Asignación por modo de } \\
\text { transporte }\end{array}$ \\
\hline & & $\begin{array}{l}\text { (P4) Ubicación de la instalación } \\
\text { y elección de modo de } \\
\text { transporte }\end{array}$ & $\begin{array}{l}\text { (P9) Cantidad de producto por } \\
\text { arco }\end{array}$ \\
\hline & & $\begin{array}{l}\text { (P5) Ubicación de nuevos arcos } \\
\text { en las redes de transporte }\end{array}$ & \\
\hline & (Q) Demanda & (Q1) Conocida & (Q2) Incierta \\
\hline & \multirow{6}{*}{ (R) Costes } & (R1) Transporte & (R8) Almacenamiento \\
\hline & & $\begin{array}{l}\text { (R2) Construir/expandir los } \\
\operatorname{arcos}\end{array}$ & (R9) Externos \\
\hline & & $\begin{array}{l}\text { (R3) Construir/abrir la } \\
\text { instalación }\end{array}$ & $\begin{array}{l}\text { (R11) Relacionados con el } \\
\text { tiempo }\end{array}$ \\
\hline & & (R4) Cierre de la instalación & $(\mathrm{R} 12)$ \\
\hline & & $\begin{array}{l}\text { (R5) Fijos en la instalación } \\
\text { (R6) Operativos en la } \\
\text { instalación }\end{array}$ & $\begin{array}{l}\text { Confiabilidad/interrupción en la } \\
\text { instalación }\end{array}$ \\
\hline & & $\begin{array}{l}\text { (R7) Operativos entre } \\
\text { instalaciones }\end{array}$ & \\
\hline & (C) Timmo & (S1) Operativo en la instalación & (S3) De envío \\
\hline & 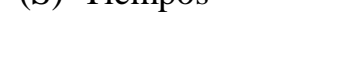 & (S2) Operativo en el transporte & (S4) De espera \\
\hline \multirow{7}{*}{ 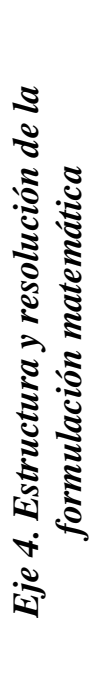 } & $\begin{array}{l}\text { (T) Tipo de } \\
\text { optimización }\end{array}$ & (T1) Minimización & (T2) Maximización \\
\hline & (U) Función objetivo & $\begin{array}{l}\text { (U1) Mono-objetivo } \\
\text { (U2) Multi-objetivo }\end{array}$ & $\begin{array}{l}\text { (U3) Multi-objetivo multi- } \\
\text { criterio }\end{array}$ \\
\hline & & (V1) Limitaciones comunes & (V5) Inventarios \\
\hline & & $\begin{array}{l}\text { (V2) Capacidad de la } \\
\text { instalación }\end{array}$ & $\begin{array}{l}\text { (V6) Presupuesto de inversión } \\
\text { (V7) Emisiones GEI }\end{array}$ \\
\hline & (V) Restricciones & $\begin{array}{l}\text { (V3) Tiempo de servicio de } \\
\text { envío }\end{array}$ & (V8) Instalaciones a localizar \\
\hline & & $\begin{array}{l}\text { (V4) Tiempo operativo de } \\
\text { transporte }\end{array}$ & \\
\hline & (W) Periodos & (W1) Único & (W2) Múltiple \\
\hline
\end{tabular}




\begin{tabular}{cll}
\hline $\begin{array}{l}\text { (X) Tipo de } \\
\text { programación } \\
\text { matemática }\end{array}$ & - PLE Programación lineal entera & $\begin{array}{l}\text { (X3) Programación entera mixta } \\
\text { - PEM }\end{array}$ \\
\hline $\begin{array}{l}\text { (X2) Programación lineal entera } \\
\text { mixta - PLEM }\end{array}$ & $\begin{array}{l}\text { (X4) Programación entera mixta } \\
\text { no-lineal - PEMNL }\end{array}$ \\
$\begin{array}{c}\text { (Y) Procedimiento de } \\
\text { resolución }\end{array}$ & $\begin{array}{l}\text { (Y1) Procedimientos de } \\
\text { relajación }\end{array}$ & $\begin{array}{l}\text { (Y3) Herramientas } \\
\text { informáticas/solucionadores }\end{array}$ \\
& (Y2) Técnicas de programación & \\
\hline
\end{tabular}

Fuente: Adaptado de Agámez-Arias et al. (2021)

\subsubsection{Resultados de la aplicación de la RSL-2EV}

En esta sección se aportan los resultados obtenidos de la aplicación de la RSL-2EV, de acuerdo con las características clave listadas en la Tabla 4.3. presentada en la subsección anterior. Para ello, se distinguen, en el apartado 4.4.2.1. los resultados con enfoque en la cadena de transporte y en el apartado 4.4.2.2. los resultados con enfoque en la cadena de suministro ${ }^{33}$.

\subsubsection{Cadena de transporte}

En la Tabla 4.4. se presentan los hallazgos de la literatura con enfoque en la cadena de transporte. En este enfoque de red no se especifican los actores involucrados en la toma de decisiones, sin embargo, es posible deducir la participación de operadores de las terminales y de transporte, incluyendo los operadores de transporte de corta distancia. Particularmente, Zhang et al. (2015) consideran los actores gubernamentales. Generalmente, los flujos de transporte se modelan como pares de origen-destino, en los que cada nodo de la red se considera como origen y destino; en los otros casos, los flujos son dirigidos con la intención de estudiar el potencial exportador de un producto/región o para evaluar el cumplimiento en los plazos de entrega. El enfoque intermodal se consideró de tres formas: a) para fomentar la actividad intermodal localizando instalaciones puntuales, b) para potenciar los efectos de las economías de escala entre las instalaciones, y c) para restringir el modo a elegir y la asignación entre las instalaciones.

En general, los modos de transporte por carretera y ferrocarril son la base para el diseño de las redes. El modo aéreo se utiliza en algunos sectores como el de servicios de paquetería, y el modo fluvial depende de las características geomorfológicas del área estudiada. Si bien el modo marítimo no se considera en las redes, si se asumen tiempos y costes de transporte para los productos disponibles en los puertos marítimos. Los productos como la soja en el sector agrícola, los pequeños envíos en los servicios de paquetería y la carga containerizada con uno o múltiples productos modelan los flujos de transporte. En países como Brasil el propósito con el diseño de la red es integrarla o expandirla decidiendo la localización de instalaciones puntuales. En los otros países, por el contrario, pretenden mejorar la eficiencia del servicio optimizando la infraestructura existente.

La planificación combinada pretende la localización de instalaciones tipo hubs, terminales intermodales y centros de integración logística. La consolidación, desconsolidación y

\footnotetext{
${ }^{33}$ Ibíd 32.
} 
clasificación son servicios inherentes a los hubs. Con el propósito de evaluar la concentración de carga, en estas instalaciones usualmente no se limita la capacidad de operación. En las terminales intermodales y los centros de integración logística destacan los servicios de transbordo y almacenamiento, especialmente en los segundos se ofrecen servicios de apoyo a los operadores (aparcamiento, restaurantes, etc.). A diferencia de lo que sucede en los hubs, limitar la capacidad permite, por ejemplo, evaluar posibles cuellos de botella en la red. Generalmente, se identifican las potenciales localizaciones para establecer en un espacio de localización discreto, la apertura (uso) de la instalación. La decisión de localización puede ir acompañada de la elección del modo y expansión de la capacidad del nodo o mejora del arco.

En su mayoría la asignación en estas redes es múltiple. La asignación de los flujos se relaciona con los servicios ofrecidos por las instalaciones, y si además están implícitas las características de la unidad o vehículos de carga, es porque la elección del modo está asociada a la decisión. Los atributos de asignación más habituales son los costes de transporte y de transbordo, siendo la distancia euclidiana (Rothenbächer et al., 2016), lineal y de economía las aplicadas para calcular los primeros (Santos et al., 2015). Los tiempos de despacho, de transporte, de servicio en la instalación y los de espera también son determinantes. Los tiempos contribuyen a la selección óptima de la configuración intermodal de la red. De manera puntual, la velocidad de circulación, la capacidad del vehículo y las emisiones GEI son otros atributos importantes para cálculo del coste y el tiempo.

Para el modelado, los costes de transporte y transbordo generalmente se consideran por unidad de carga y modo, o como fracciones en función del coste generado en cada sección de la red. Adicionalmente, Ishfaq \& Sox (2012) incorporan los costes operativos en la instalación (carga/descarga y consolidación/desconsolidación). Los costes de construir/abrir las instalaciones y los costes fijos usualmente no se consideran. La consideración de criterios medioambientales para definir las alternativas de solución es una preocupación relativamente reciente. Aunque en su mayoría refieren a las emisiones GEI, la congestión, el tráfico y la contaminación del aire y acústica también se consideran. Los aspectos medioambientales inciden tanto en la elección del modo como en las decisiones de localización y asignación ya que estos se integran al modelo como costes. Los modelos son de naturaleza determinista donde la demanda y los parámetros son conocidos.

En estos problemas es común la convergencia de varios objetivos, pero a efectos de unificar la formulación, se emplean métricas para establecer una función objetivo que minimice los costes. Ejemplo de ello son las propuestas de Amaral et al. (2012), Santos et al. (2015) y Zhang et al. (2013). Las principales restricciones están relacionadas con el número de instalaciones, la asignación entre las instalaciones, y el máximo flujo en nodos y arcos. Otras limitaciones dependen de aspectos particulares del problema, como son los tiempos de servicio, el presupuesto de inversión y las emisiones máximas permitidas (Kim et al., 2013). El análisis de escenarios compensa las escasas propuestas en contextos de múltiples periodos. Los métodos exactos priman en la resolución del modelo, entre estos los algoritmos de rama y precio y corte, y de ramificación y acotación. En otros casos la complejidad matemática requiere el uso de técnicas de relajación como las desigualdades validas, o el desarrollo de métodos de solución aproximada como algoritmos genéticos y heurísticas simples. Para comparar el rendimiento de los procedimientos propuestos se utilizan los solucionadores CPLEX, GUROBI, BONMIN y GNU GLPK. 
Tabla 4.4. Resultados de la RSL-2EV con enfoque en la cadena de transporte

\begin{tabular}{|c|c|c|c|c|}
\hline Referencias & Eje 1 & Eje 2 & Eje 3 & Eje 4 \\
\hline $\begin{array}{l}\text { Alumur et al. } \\
\text { (2012) }\end{array}$ & $\begin{array}{l}\text { C1, D4, E3, F2, } \\
\text { G4, I8 }\end{array}$ & $\begin{array}{l}\mathrm{J} 5, \mathrm{~K} 2, \mathrm{~L} 1, \mathrm{M} 1, \\
\mathrm{M} 2, \mathrm{M} 3, \mathrm{M} 4, \mathrm{~N} 1, \\
\mathrm{O} 1, \mathrm{O} 2, \mathrm{O} 3, \mathrm{O} 4\end{array}$ & $\begin{array}{l}\text { P1, P6, P7, Q1, R1, } \\
\text { R3, R5, R6, R7, } \\
\text { S1, S2, S3 }\end{array}$ & $\begin{array}{l}\text { T1, U2, V1, V3, } \\
\text { V4, W1, X2 }\end{array}$ \\
\hline $\begin{array}{l}\text { Amaral et al. } \\
\text { (2012) }\end{array}$ & $\begin{array}{l}\text { B1, B2, D1, E4, } \\
\text { F5, G1, H3, I3 }\end{array}$ & $\begin{array}{l}\text { J6, K1, L2, M4, } \\
\text { N2, O1, O2, O5 }\end{array}$ & P1, P9, Q1, R1, R6 & $\begin{array}{l}\text { T1, U1, V1, W1, } \\
\text { X1 }\end{array}$ \\
\hline $\begin{array}{l}\text { Guimarães et al. } \\
\text { (2017) }\end{array}$ & $\begin{array}{l}\text { D1, E2, F5, G1, } \\
\text { H3, I3 }\end{array}$ & $\begin{array}{l}\mathrm{J} 2, \mathrm{~K} 1, \mathrm{~L} 2, \mathrm{M} 1, \\
\mathrm{M} 2, \mathrm{M} 3, \mathrm{M} 4, \mathrm{M} 6, \\
\mathrm{~N} 2, \mathrm{O} 1, \mathrm{O} 2, \mathrm{O} 3\end{array}$ & $\begin{array}{l}\text { P1, P9, Q1, R1, } \\
\text { R5, R6 }\end{array}$ & $\mathrm{T} 1, \mathrm{U} 2, \mathrm{~V} 1, \mathrm{X} 2$ \\
\hline $\begin{array}{l}\text { Ishfaq \& Sox } \\
\text { (2012) }\end{array}$ & $\begin{array}{l}\text { D4, E3, F3, G3, } \\
\text { H7, I5 }\end{array}$ & $\begin{array}{l}\mathrm{J} 5, \mathrm{~K} 2, \mathrm{~L} 1, \mathrm{M} 1, \\
\mathrm{M} 2, \mathrm{M} 3, \mathrm{M} 4, \mathrm{~N} 2, \\
\mathrm{O} 1, \mathrm{O} 4\end{array}$ & $\begin{array}{l}\text { P3, P9, Q1, R1, } \\
\text { R3, R5, R6, S2, } \\
\text { S3, S4 }\end{array}$ & $\begin{array}{l}\text { T1, U2, V1, V3, } \\
\text { V4, W1, X2 }\end{array}$ \\
\hline Kim et al. (2013) & $\begin{array}{l}\text { D4, E4, F2, G3, } \\
\text { H7, I4 }\end{array}$ & $\begin{array}{l}\mathrm{J} 6, \mathrm{~J} 8, \mathrm{~K} 1, \mathrm{~L} 1, \mathrm{M} 4 \\
\mathrm{O} 1, \mathrm{O} 2, \mathrm{O} 3, \mathrm{O} 4 \\
\text { O7 }\end{array}$ & $\begin{array}{l}\text { P1, P8, Q1, R1, } \\
\text { R2, S2 }\end{array}$ & $\begin{array}{l}\text { T1, U2, V6, V7, } \\
\text { X4 }\end{array}$ \\
\hline $\begin{array}{l}\text { Rahmaniani \& } \\
\text { Ghaderi (2013) }\end{array}$ & $\begin{array}{l}\text { B1, B8, C1, D3, } \\
\text { E4, F8, H7 }\end{array}$ & $\begin{array}{l}\mathrm{J} 9, \mathrm{~L} 2, \mathrm{M} 4, \mathrm{~N} 2, \\
\mathrm{O} 1, \mathrm{O} 8\end{array}$ & $\begin{array}{l}\text { P1, P5, Q1, R1, } \\
\text { R2, R3 }\end{array}$ & $\begin{array}{l}\text { T1, U2, V1, V6, } \\
\text { W1, Y2 }\end{array}$ \\
\hline $\begin{array}{l}\text { Rahmaniani \& } \\
\text { Ghaderi (2015) }\end{array}$ & $\begin{array}{l}\text { B1, B8, C1, D3, } \\
\text { E4, F8, H7 }\end{array}$ & $\begin{array}{l}\text { J9, K1, L2, M4, } \\
\text { N2, O1 }\end{array}$ & $\begin{array}{l}\text { P2, P5, Q1, R1, } \\
\text { R2, R3, R5 }\end{array}$ & $\begin{array}{l}\text { T1, U2, V1, W1, } \\
\text { Y2 }\end{array}$ \\
\hline $\begin{array}{l}\text { Rothenbächer et } \\
\text { al. (2016) }\end{array}$ & $\begin{array}{l}\text { D3, E4, F2, G3, } \\
\text { H7, I1 }\end{array}$ & $\begin{array}{l}\mathrm{J} 5, \mathrm{~K} 2, \mathrm{~L} 1, \mathrm{M} 1, \\
\mathrm{M} 2, \mathrm{M} 3, \mathrm{M} 4, \mathrm{~N} 2, \\
\mathrm{O} 1, \mathrm{O} 2, \mathrm{O} 4, \mathrm{O} 5\end{array}$ & $\begin{array}{l}\mathrm{P} 1, \mathrm{P} 9, \mathrm{Q} 1, \mathrm{R} 1, \\
\mathrm{R} 6, \mathrm{~S} 2, \mathrm{~S} 3\end{array}$ & $\mathrm{~T} 1, \mathrm{U} 2, \mathrm{~V} 1, \mathrm{X} 3$ \\
\hline $\begin{array}{l}\text { Santos et al. } \\
\text { (2015) }\end{array}$ & $\begin{array}{l}\text { D4, E4, F2, G3, } \\
\text { H7, I2 }\end{array}$ & $\begin{array}{l}\mathrm{J} 5, \mathrm{~K} 2, \mathrm{~L} 2, \mathrm{M} 1, \\
\mathrm{M} 2, \mathrm{M} 3, \mathrm{M} 4, \mathrm{~N} 2, \\
\mathrm{O} 1, \mathrm{O} 2, \mathrm{O} 3, \mathrm{O} 5\end{array}$ & $\begin{array}{l}\text { P1, P9, Q1, R1, } \\
\text { R5, R6, R9 }\end{array}$ & $\mathrm{T} 1, \mathrm{U} 2, \mathrm{~V} 1, \mathrm{X} 3$ \\
\hline $\begin{array}{l}\text { Zhang et al. } \\
\text { (2013) }\end{array}$ & $\begin{array}{l}\text { D4, E4, F5, G3, } \\
\text { H7, I6 }\end{array}$ & $\begin{array}{l}\text { J6, K1, L2, M4, } \\
\text { O2, O3, O7 }\end{array}$ & $\begin{array}{l}\text { P1, P8, P9, Q1, R1, } \\
\text { R6, R10, R11 }\end{array}$ & $\begin{array}{l}\text { T1, U3, V1, Y2, } \\
\text { Y3 }\end{array}$ \\
\hline $\begin{array}{l}\text { Zhang et al. } \\
\text { (2015) }\end{array}$ & 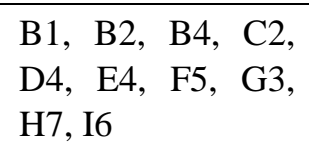 & $\begin{array}{l}\text { J6, K1, L1, M4, } \\
\text { N1, O1, O2, O3, } \\
\text { O4, O5, O7 }\end{array}$ & $\begin{array}{l}\mathrm{P} 1, \mathrm{P} 8, \mathrm{Q} 1, \mathrm{R} 1, \\
\mathrm{R} 6, \mathrm{R} 10, \mathrm{R} 11, \mathrm{~S} 2\end{array}$ & $\begin{array}{l}\text { T1, U2, V6, Y2, } \\
\text { Y3 }\end{array}$ \\
\hline
\end{tabular}

Fuente: Adaptado de Agámez-Arias et al. (2021)

\subsubsection{Cadena de suministro}

La Tabla 4.5. presenta los hallazgos más relevantes sobre la cadena de suministro en los problemas analizados. Proveedores, fabricantes, distribuidores y minoristas son los actores identificados en la cadena de suministro contemplando, generalmente, un solo actor por tipo. No obstante, Davidson \& Leachman (2012) para evaluar riesgos en los tiempos de entrega incorporan múltiples distribuidores, y Fernandes et al. (2013) para evaluar los niveles de colaboración y el uso compartido de la infraestructura consideran múltiples actores por tipo. Los flujos en la red son dirigidos, a través de ellos y en conjunto con la localización de instalaciones intermodales, se modelan los efectos de las interrupciones, cambios en la 
demanda y la demanda insatisfecha. A diferencia de lo que sucede en las redes de transporte, el modo marítimo se incluye, pero con atributos en las decisiones de asignación; también destacan los modos por carretera y ferroviario. El sector de minería y energía de Estados Unidos inspiró varios de los estudios analizados en este apartado, por ejemplo, Hajibabai \& Ouyang, (2013) y Xie et al. (2014). Las aplicaciones van desde el suministro de biomasa a la distribución de biocombustibles y derivados del petróleo como etanol, gasolina, diésel y combustible de aviación.

En la cadena de suministro se localizan instalaciones tipo fábrica, centro de distribución, depósito, hub y terminal intermodal. Los dos últimos tipos se conciben como puntos intermedios entre proveedores y fábricas para la consolidación del producto. Las fábricas son refinerías y biorrefinerías donde se transforma el petróleo crudo y la biomasa. En función de la estacionalidad del producto, las interrupciones en la red y las estrategias de distribución se toman decisiones para la localización de terminales intermodales, centros de distribución o depósitos, cuyos servicios principales son transbordo, consolidación/desconsolidación, almacenamiento y gestión de inventarios. Las instalaciones intermodales, además de reducir los costes de inventario, facilitan la conexión con los demás eslabones de la cadena. No obstante, los modelos tienden a considerar una mayor variedad de decisiones en las instalaciones, tales como: apertura, operación y cierre de la instalación, niveles de producción, recursos de transporte o demanda insatisfecha.

La capacidad normalmente se establece como una restricción en todas las instalaciones, pero en ocasiones puntuales no supone una limitación real ya que los flujos no exceden el límite. Entre los atributos de asignación, las distancias se utilizan como base para el cálculo de los costes de transporte, ya sea estableciendo una relación directa o empleando distancias de Hamming. Otras características diferenciadoras están relacionadas con los costes de inventario, la estrategia de distribución elegida para los despachos (directos o intermodales) y los pedidos satisfechos. Los tiempos de procesamiento y reabastecimiento, especialmente en la materia prima, así como los tiempos operativos de transporte y de servicio, las velocidades de tránsito y la capacidad de los vehículos también condicionan la toma de decisiones combinada.

Para el modelado, además de los costes habituales de localizar las instalaciones y los de transporte, los costes de inventario, por demanda insatisfecha, por interrupciones en los arcos de la red, producción, almacenamiento, entre otros, son igualmente importantes para el diseño. La incorporación de los tiempos de transporte permite modelar la aleatoriedad en la prestación del servicio, los tiempos de reaprovisionamientos tienen efecto en los ciclos de inventario y en los tiempos de procesamiento en la fabricación. Generalmente la demanda es de tipo determinista, sin embargo, la incertidumbre en la demanda se ha asociado a los modelos que consideran los inventarios.

Las formulaciones propuestas implican la toma de decisiones de múltiples objetivos cuyas funciones optimizan los valores mínimos de los objetivos. De manera excepcional, Fernandes et al. (2013) maximizan los beneficios en los múltiples períodos analizados. Debido a las características específicas incluidas en cada uno de los problemas de estudio no es posible definir restricciones comunes. Algunas limitaciones particulares son la cantidad de productos a fabricar, disponibilidad de la materia prima, la capacidad de las instalaciones y el almacenamiento del producto. En contextos de múltiples periodos, las decisiones de localización de las instalaciones se mantienen en todo el horizonte de planificación. Los PLEM 
y PEMNL son los tipos de programación propuestos. Las técnicas de resolución incluyen la descomposición de Benders, relajación lagrangiana, heurísticas, la utilización de sistemas de información geográfica como ArcGIS y solucionadores comerciales.

Tabla 4.5. Resultados de la RSL-2EV con enfoque en la cadena de suministro

\begin{tabular}{|c|c|c|c|c|}
\hline Referencias & Eje 1 & Eje 2 & Eje 3 & Eje 4 \\
\hline $\begin{array}{l}\text { Alenezi \& } \\
\text { Darwish (2014) }\end{array}$ & $\begin{array}{l}\text { B5, B7, B8, B9, } \\
\text { D3, E2, F2, H7 }\end{array}$ & $\begin{array}{l}\mathrm{J} 3, \mathrm{~J} 7, \mathrm{~J} 8, \mathrm{~K} 2, \mathrm{~L} 1, \\
\mathrm{M} 2, \mathrm{M} 4, \mathrm{~N} 1, \mathrm{O} 1, \\
\mathrm{O} 4, \mathrm{O} 6\end{array}$ & $\begin{array}{l}\text { P1, P6, P9, Q2, R1, } \\
\text { R3, R8, S3 }\end{array}$ & $\begin{array}{l}\text { T1, U2, V1, W1, } \\
\text { X4 }\end{array}$ \\
\hline $\begin{array}{l}\text { Brahami et al. } \\
(2020)\end{array}$ & $\mathrm{C} 2, \mathrm{D} 3, \mathrm{H} 7$ & $\begin{array}{l}\text { J8, J9, K1, L2, M2, } \\
\text { M4, N2, O1, O2, } \\
\text { O7 }\end{array}$ & $\begin{array}{l}\text { P1, P5, P6, Q1, R1, } \\
\text { R2 }\end{array}$ & $\begin{array}{l}\text { T1, U3, V2, V6, } \\
\text { V7, V8, W1, X3, } \\
\text { Y2 }\end{array}$ \\
\hline $\begin{array}{l}\text { Davidson \& } \\
\text { Leachman (2012) }\end{array}$ & $\begin{array}{l}\text { B7, B8, B9, C2, } \\
\text { D2, E2, F4, H7, I5 }\end{array}$ & $\begin{array}{l}\text { J6, K2, L1, M2, } \\
\text { M3, M4, M5, M8, } \\
\text { N1, O1, O2, O4, } \\
\text { O6 }\end{array}$ & $\begin{array}{l}\text { P1, P8, Q2, R1, } \\
\text { R6, R8, R11, S1, } \\
\text { S2, S3 }\end{array}$ & $\begin{array}{l}\text { T1, U2, V1, V5, } \\
\text { W1, X4 }\end{array}$ \\
\hline $\begin{array}{l}\text { Fernandes et al. } \\
\text { (2013) }\end{array}$ & $\begin{array}{l}\text { B8, B9, C2, D2, } \\
\text { E4, F7, G2, H5, I7 }\end{array}$ & $\begin{array}{l}\mathrm{J} 1, \mathrm{~K} 1, \mathrm{~L} 2, \mathrm{M} 4, \\
\mathrm{~N} 2, \mathrm{O} 1, \mathrm{O} 2, \mathrm{O} 4\end{array}$ & $\begin{array}{l}\text { P1, P3, P8, Q1, R1, } \\
\text { R3, R5, R8, S1, S2 }\end{array}$ & $\begin{array}{l}\text { T2, U2, V1, V5, } \\
\text { W2, X2 }\end{array}$ \\
\hline $\begin{array}{l}\text { Hajibabai \& } \\
\text { Ouyang (2013) }\end{array}$ & $\begin{array}{l}\text { B5, B8, B9, D3, } \\
\text { E4, F2, G2, H2, I5 }\end{array}$ & $\begin{array}{l}\text { J4, J8, K1, L2, M7, } \\
\text { N1, O1, O2, O4 }\end{array}$ & $\begin{array}{l}\text { P1, P6, P9, Q1, R2, } \\
\text { R3, S2 }\end{array}$ & $\begin{array}{l}\text { T1, U2, V1, W1, } \\
\text { X4 }\end{array}$ \\
\hline $\begin{array}{l}\text { Kazemi \& } \\
\text { Szmerekovsky } \\
\text { (2015) }\end{array}$ & $\begin{array}{l}\text { B8, B9, D2, E4, } \\
\text { F6, G2, H6, I5 }\end{array}$ & $\begin{array}{l}\mathrm{J} 3, \mathrm{~K} 1, \mathrm{~L} 2, \mathrm{M} 4, \\
\mathrm{O} 1, \mathrm{O} 2\end{array}$ & P1, P9, Q1, R3 & $\begin{array}{l}\text { T1, U2, V1, V2, } \\
\text { W1, X4 }\end{array}$ \\
\hline $\begin{array}{l}\text { Marufuzzaman et } \\
\text { al. (2014) }\end{array}$ & $\begin{array}{l}\text { B5, B6, B8, D1, } \\
\text { E4, F5, G2, H1, I5 }\end{array}$ & $\begin{array}{l}\text { J4, J5, K1, L1, M2, } \\
\text { M7, N1, O1, O2, } \\
\text { O3, O5 }\end{array}$ & $\begin{array}{l}\text { P1, P8, P9, Q2, R3, } \\
\text { R6 }\end{array}$ & T1, U2, W1, X2 \\
\hline $\begin{array}{l}\text { Shishebori \& } \\
\text { Jabalameli (2013) }\end{array}$ & $\begin{array}{l}\text { B7, C1, D3, F8, } \\
\text { H7 }\end{array}$ & $\begin{array}{l}\text { J3, K2， L2， M2, } \\
\text { N2, O1 }\end{array}$ & $\begin{array}{l}\text { P1, P5, P6, Q1, R1, } \\
\text { R2, R12, }\end{array}$ & $\begin{array}{l}\text { T1, U2, V1, V8, } \\
\text { W1, X4, Y3 }\end{array}$ \\
\hline Xie et al. (2014) & $\begin{array}{l}\text { B5, B8, B9, D3, } \\
\text { E3, F2, G2, H2, I5 }\end{array}$ & $\begin{array}{l}\mathrm{J} 4, \mathrm{~J} 5, \mathrm{~J} 6, \mathrm{~K} 1, \mathrm{~L} 2, \\
\mathrm{M} 2, \mathrm{M} 4, \mathrm{M} 5, \mathrm{M} 7, \\
\mathrm{~N} 1, \mathrm{O} 1, \mathrm{O} 2, \mathrm{O} 3, \\
\mathrm{O} 4, \mathrm{O} 5, \mathrm{O} 6\end{array}$ & $\begin{array}{l}\text { P2, P9, Q1, R3, } \\
\text { R5, R6, R8 }\end{array}$ & $\mathrm{T} 1, \mathrm{U} 2, \mathrm{~W} 2, \mathrm{X} 3$ \\
\hline
\end{tabular}

Fuente: Adaptado de Agámez-Arias et al. (2021)

\subsection{Implicaciones del trabajo}

El transporte intermodal en un tema de investigación con un interés vigente y creciente, cuya evolución y aplicabilidad ha suscitado el surgimiento de términos empleados como sinónimos que albergan diferencias referidas a la configuración modal y las distancias, la seguridad y flexibilidad del sistema, o la sincronía y coordinación entre los actores y actividades del sistema. Estas diferencias por si mismas no conllevan a limitaciones para agrupar la literatura que se consideró en la RSL y la RSL-2EV pues el marco de acción establecido en ambas revisiones, basado en los problemas de localización de instalaciones y diseño de la red, generan 
una desvinculación automática, por ejemplo, de la literatura referida al transporte co-modal. Con la distinción de los problemas de decisión, bien sea por el análisis individual o combinado, se determinó la influencia de los factores clave en la eficiencia del sistema y la calidad del servicio. En los primeros, la visión tradicional centrada en aspectos económicos se está transformando a una visión que integra las tres dimensiones de la sostenibilidad. En los segundos, se acentúa la importancia de la capacidad y el nivel de servicio en las terminales intermodales, así como el uso de tecnología, los sistemas de información y la coordinación entre los actores.

Para el análisis de tales factores las técnicas de programación matemática tienen un papel diferenciado para la adaptación de las situaciones del mundo real. Con la aplicación de estas técnicas es posible identificar un conjunto de variantes para cada uno de los problemas de decisión que se combina en los LI-DR, como son: los problemas de localización-asignación de instalaciones, localización de instalaciones en la red, diseño de la red vial, diseño de la red de transporte público y los problemas de localización-enrutamiento. Si bien algunos de estos tienden a la planificación simultánea estratégico-táctica, distan de los problemas combinados LI-DR porque, los de localización de instalaciones se concentran en la configuración de la red de instalaciones y los de diseño de red en la configuración de las redes de transporte. Por tanto, la combinación de las decisiones conduce a un elevado número de factores clave, y diversos tomadores de decisiones y grupos de interés que precisan objetivos incompatibles.

Los resultados de los RSL-2EV demuestran que la naturaleza integradora de los problemas combinados LI-DR ha superado la barrera de aplicación al entorno de la cadena de transporte para introducirse en la toma de decisiones de la cadena de suministro. En las cadenas de transporte los organismos gubernamentales, además de financiadores de las infraestructuras, están siendo incluidos como actores principales en la toma de decisiones; mientras que, en las cadenas de suministro, los actores de la cadena de transporte se están considerando como sujetos pasivos en la toma de decisiones. En general, los modos por carretera y ferroviario son los más utilizados, sin embargo, las características del producto que se transporta y el sector económico determinan la consideración de otros modos como el aéreo. El transporte marítimo no se considera como opción de configuración modal, este se puede considerar un resultado coherente debido a que la localización de arcos en la red implica la transformación interna de un territorio. Además, el propósito del diseño de la red también contempla la optimización en el uso de la infraestructura existente.

Las decisiones de asignación están determinadas principalmente por la capacidad de las instalaciones, costes y tiempos de transporte, velocidad del tráfico, distancias y emisiones, pero difieren significativamente de las decisiones de cadena de suministro. En las cadenas de transporte, pese a que se consideran varios servicios logísticos, es usual que se modelen como un único servicio, mientras, en las cadenas de suministro se presentan de manera desagregada debido a que se ofrecen en distintas etapas de la misma. Los problemas se modelan en su mayoría como PNLEM y PLEM y generalmente incluyen las redes de transporte subyacentes. Desde el enfoque de la cadena de transporte el planteamiento asocia decisiones de mejora en los arcos. Desde el enfoque de la cadena de suministro, enfatiza en la elección del modo a través de parámetros de distancia, velocidad y tiempos. El objetivo principal que impulsa el diseño de estas redes es minimizar costes o maximizar beneficios, no obstante, los problemas combinados LI-DR poseen un carácter funcional inherente que aporta, entre otras cosas, el cumplimiento de aspectos de política pública, flexibilidad, sostenibilidad y competitividad. 


\subsection{Conclusiones}

En este capítulo se sintetizan los resultados obtenidos del análisis de la literatura, en general, sobre los sistemas de transporte intermodal aplicando una RSL, y en particular sobre los problemas combinados LI-DR aplicando una RSL-2EV. La RSL-2EV adopta una metodología de dos etapas que fusiona una primera etapa en la que se analizaron revisiones publicadas para establecer las pautas y criterios que permitieron el posterior desarrollo de la RSL en la segunda etapa. Para direccionar el desarrollo de la segunda etapa se formularon los siguientes interrogantes: ¿cuáles son las principales características de los problemas combinados LI-DR intermodal? ¿qué aspectos se consideran en la toma de decisiones de este tipo de problema? ¿qué técnicas de programación matemática se utilizan? y, ¿qué temas emergentes se pueden identificar como futuras líneas de investigación?

Los problemas combinados LI-DR ofrecen aportes desde dos enfoques distintos de red. La revisión de la literatura confirma que los dos enfoques de investigación dominantes, la cadena de transporte y la cadena de suministro, se modelan con distintas características y adoptan diferentes perspectivas para abordar el problema combinado. Estas diferencias se extienden a la naturaleza de los modelos y formulaciones propuestos. Las cadenas de suministro suelen incluir un mayor número de decisiones, actores y parámetros de análisis. Las cadenas de transporte apuestan por un servicio más eficiente considerando la consolidación y la sostenibilidad, principalmente, medioambiental. La capacidad del sistema desde cada enfoque establece límites para diseñar y rediseñar redes intermodales flexibles. Los resultados han demostrado la eficacia de la programación matemática para analizar los problemas combinados LI-DR. La convergencia de dos niveles de planificación (estratégico y táctico), la elección del modo de transporte y las características implícitas en cada enfoque de red enriquecen el conjunto de técnicas heurísticas y metaheurísticas que se pueden utilizar para analizar este tipo de problemas.

Las futuras líneas de investigación identificadas arrojan oportunidades en distintas direcciones. Entre estas, puede ser atractivo analizar la colaboración y cooperación entre los actores y la sincronización de las actividades y procesos entre las etapas de la cadena. También parece interesante apostar por el desarrollo de técnicas que respondan rápidamente a la optimización personalizada y de escala de los datos del modelo. La incertidumbre de los datos asociados a los diferentes escenarios de ocurrencia para evaluar la viabilidad y optimización de soluciones tanto como los efectos en la volatilidad del presupuesto de inversión también pueden ser líneas prometedoras para explorar y desarrollar. Igualmente, es razonable formular modelos multi-periodo y multi-objetivo multi-criterio que contemplen las necesidades productivas, socioeconómicas y medioambientales de la zona de estudio. Con los modelos multi-periodo es posible analizar los efectos de la disponibilidad de los recursos financieros para diseñar de la red intermodal, así como la interdependencia en las decisiones y los múltiples presupuestos de inversión. Para los modelos multi-objetivo multi-criterio es posible aprovechar las características geográficas y socioeconómicas de la zona de estudio para valorar los efectos en las compensaciones provocados por las preferencias definidas para diseñar la red intermodal.

En los siguientes capítulos se proponen modelos de programación matemática (2 versiones que se representan en 5 variantes) para contribuir a algunas de estas líneas de investigación. Por una parte, se proponen modelos que permiten analizar los efectos de las limitaciones de 
financiación en las decisiones de diseño de la red intermodal para un único periodo de decisión. Luego se extiende la formulación a un modelo multi-periodo y se valida la interdependencia en las decisiones y la disponibilidad de múltiples presupuestos de inversión. Por último, se propone un modelo multi-objetivo multi-criterio para analizar el grado de conflicto de las decisiones, los efectos en el diseño sostenible de la red intermodal y las estrategias de preferencia de los decisores. En estas propuestas también se tienen en cuenta otros aspectos propios del problema descrito en el capítulo 3. 


$$
\text { CApítulO } 5
$$




\title{
Capítulo 5
}

\section{Diseño económico de la red de transporte intermodal: propuesta de un modelo de programación lineal entero- mixto mono-periodo}

\begin{abstract}
Resumen: en este capítulo se desarrolla y utiliza un modelo de programación lineal enteromixto (MPLEM) mono-periodo para dar respuesta, desde un enfoque económico, al problema combinado de localización de instalaciones-diseño de red (LI-DR) descrito en el capítulo 3. El MPLEM incorpora las lagunas identificadas en la literatura respecto a la planificación endógena de la capacidad del sistema y la importancia de los costes como factores clave de las alternativas de solución. El modelo se resuelve con el esquema de resolución R+Gurobi (descrito en la subsección 3.5.1.) y se analiza el comportamiento de la solución de diseño económico de la red de transporte intermodal (DERTI). El análisis, si bien refleja un comportamiento esperado y aceptable, deja al descubierto los desequilibrios ocasionados por las pérdidas de producto que se ocasionan en los nodos de origen de la zona de estudio. A partir de este resultado se valida el modelo incorporando una nueva restricción para limitar las pérdidas en cada nodo de origen. Los resultados demuestran la utilidad del modelo a la vez que proporcionan información vital para guiar la toma de decisiones de las administraciones públicas.
\end{abstract}




\subsection{Introducción}

El estado del arte presentado en el capítulo 4 describe los factores que influyen en la valoración de la eficiencia económica y la calidad del servicio en los sistemas intermodales, entre los cuales destacan la capacidad del sistema y los costes. Los resultados de la revisión sistemática de la literatura en dos etapas vinculadas (RSL-2EV) son concluyentes respecto a que en los problemas combinados de localización de instalaciones-diseño de red (LI-DR) es poco frecuente modelar las capacidades del sistema, así como algún tipo de limitación de financiación. Los estudios que consideraron la capacidad, ésta fue definida de manera exógena, establecida como un parámetro del modelo, principalmente, para las instalaciones de la red. En los arcos de la red las decisiones se han centrado mayoritariamente en el uso de los arcos existentes para asignar los flujos de transporte y los vehículos modales. Por su parte, las limitaciones de financiación no se han considerado extensivamente, pues, generalmente, los costes de inversión se integran entre los criterios que optimizan la solución del problema.

En este capítulo se desarrolla y utiliza un modelo de programación lineal entero-mixto (MPLEM) mono-periodo para dar respuesta, desde un enfoque económico, al problema combinado LI-DR descrito en el capítulo 3. El MPLEM incorpora las lagunas identificadas en la literatura respecto a la planificación endógena de la capacidad del sistema y la importancia de los costes como factores clave de las alternativas de solución. El modelo se resuelve con el esquema de resolución R+Gurobi (descrito en la subsección 3.5.1.) y se analiza el comportamiento de la solución de diseño económico de la red de transporte intermodal (DERTI) cambiando los valores de la demanda, la capacidad de las instalaciones intermodales y el presupuesto de inversión disponible. Este análisis pone de manifiesto los efectos de las pérdidas de producto en los nodos de origen, por lo que se valida el modelo introduciendo una nueva restricción que limita las pérdidas en estos nodos.

El capítulo está organizado de la siguiente manera. En la sección 5.2. se presenta una revisión de la literatura sobre las decisiones de capacidad en las instalaciones y las limitaciones al presupuesto de inversión. En la sección 5.3. se formula el MPLEM mono-periodo con enfoque económico. En la sección 5.4. se presenta el análisis de la solución en dos sentidos: los costes óptimos de uso de la red y las soluciones de DERTI. Los resultados de los análisis conllevan a validar la formulación incorporando una nueva restricción al modelo. Los resultados de la validación se presentan en la subsección 5.4.4. Las secciones 5.5. y 5.6. aportan, respectivamente, la discusión y las conclusiones de este capítulo.

\subsection{Revisión de la literatura}

Para formular un MPLEM mono-periodo que contribuya a cerrar la brecha de las lagunas identificadas en los problemas combinados LI-DR intermodal, se realiza en esta sección una revisión de la literatura relacionada con la localización de instalaciones y el diseño de la red. La literatura se revisa con el fin de determinar las formas de modelado empleadas para decidir la capacidad en las instalaciones y definir la limitación de financiación. En las subsecciones 5.2.1. y 5.2.2. se exponen, respectivamente, las formas de modelado encontradas. 


\subsubsection{Planificación endógena de la capacidad en las instalaciones}

La capacidad de las instalaciones es una decisión de nivel estratégico que tiene un impacto en la configuración física de la red de instalaciones, así como en las decisiones tácticas de asignación de la demanda, rutas de servicio, flujos y modos de transporte (Crainic \& Kim, 2007). La planificación endógena de la capacidad se ha estudiado con características continuas y discretas. En las continuas, la capacidad se expande o transfiere continuamente y cada instalación, nueva o existente, está limitada a un nivel máximo para acoger las mejoras o cambios (Jena et al., 2015). Las decisiones de capacidad con características discretas se emplean para aprovechar los beneficios de las economías de escala y establecer los costes operativos en las instalaciones (Correia et al., 2018). Estas se modelan a partir de un conjunto de opciones definidas por niveles, tamaños o módulos (Fattahi et al., 2015). Cada opción del conjunto tiene asociado, al menos, costes para construir/abrir la instalación, costes para operarla y un límite máximo para el nivel, tamaño o módulo (Silva et al., 2021).

El límite máximo establecido para cada nivel restringe el flujo de producto que se asigna a la instalación. Lo más notable en la literatura es que se restrinjan los flujos de entrada (Correia et al., 2010). No obstante, Kazemi \& Szmerekovsky (2015) modelan los flujos de salida de las instalaciones intermodales que se localizan aguas abajo de la cadena de suministro. La decisión de capacidad se ha modelado en horizontes de planificación mono-periodo (estáticos) y multiperiodo (dinámicos). En los mono-periodo, la decisión de capacidad se toma eligiendo hasta una opción del conjunto de niveles que se proporcionan (Lee, 1991). Por su parte, cuando las decisiones se planifican para distintos periodos de decisión se puede elegir, para una misma instalación, una o varias opciones del conjunto de niveles de capacidad proporcionado (Badri et al., 2013). En esta última, lo más usual es que se establezca una secuencia para seleccionar los niveles, con la cual se determina la progresión en la construcción o uso de la instalación (Hoff et al., 2017).

Las decisiones de capacidad en las instalaciones se han modelado para diseñar y rediseñar la red. En el diseño de la red, la decisión se relaciona con la capacidad inicial de las nuevas instalaciones que se localizan (Alumur et al., 2016). Mientras, en el rediseño de la red se producen ajustes de capacidad a las instalaciones existentes, estos ajustes se asocian con la expansión, reducción o transferencia de la capacidad (Melo et al., 2009). Cuando los ajustes a la capacidad se modelan considerando los cambios de la demanda es posible introducir en el modelo otras decisiones que atañen a las instalaciones, tales como: reubicación, reapertura, cierre temporal o cierre definitivo (Silva et al., 2021). Generalmente, en los modelos que introducen la planificación endógena de la capacidad en las instalaciones se restringen las decisiones a un presupuesto de inversión (Asl et al., 2020) y son de mayor aplicación en un contexto dinámico (Silva et al., 2021). Los efectos en el diseño/rediseño de la red suelen analizarse teniendo en cuenta argumentos basados en la irreversibilidad en la construcción de las instalaciones, los costes de inversión y los cuellos de botella (Correia et al., 2018).

Para el MPLEM mono-periodo que se propone en la sección 5.3. las decisiones de capacidad en las instalaciones se modelan de manera discreta, proporcionando un conjunto de alternativas de capacidad a partir de los cuales se elige un nivel por cada instalación intermodal que se localiza. En la subsección 5.3.1. se explica en detalle cómo se modelan estas decisiones. 


\subsubsection{Limitación del presupuesto de inversión ${ }^{34}$}

Dado que la localización/construcción de nueva infraestructura (instalaciones o de transporte) conlleva incurrir en costes de inversión elevados que no se disponen de manera inmediata, las decisiones se pueden supeditar al presupuesto disponible o a las estrategias de inversión de los tomadores de decisión (Baykasoğlu et al., 2019). Una disponibilidad limitada de financiación para diseñar la red condiciona la selección (en modelos mono-periodo) o selecciónprogramación (en modelos multi-periodo) del número de proyectos que se evalúan (Hosseininasab \& Shetab-Boushehri, 2015). Ante esta limitación parece idóneo considerar diferentes formas tanto de modelar la financiación disponible, como de modelar la localización/construcción de los proyectos por etapas o fases (Melo et al., 2009). En la literatura esta limitación ha sido empleada para determinar la mejor manera de asignar los recursos económicos (Murawski \& Church, 2009) o reducir el conjunto de soluciones factibles del problema (Sanci \& Daskin, 2019); indistintamente de la forma que se emplea, para una misma disponibilidad de financiación el número de diseños factibles se amplía sustancialmente, más aún, cuando la limitación se incorpora en modelos con múltiples periodos de decisión (PeñaMora \& Li, 2001).

Por lo general, en los modelos mono-periodo la limitación de la financiación disponible se formula se manera simple, identificándose dos formas para ello. En la primera, considerada la de más frecuente aplicación, consiste en que la sumatoria de los costes de inversión de los proyectos que se seleccionan sea inferior o igual al presupuesto disponible (Crainic \& Laporte, 1997; Wiley \& Timmermans, 2009). En la segunda, se toman como referencia las distintas fases de construcción de los proyectos y el presupuesto disponible para cada fase, comprometiendo los recursos económicos de cada fase de manera automática. Con esta última se pretende garantizar que todos los proyectos seleccionados se ejecuten hasta su última fase (Bagloee et al., 2018). Dependiendo de las variables de decisión con las que se declaran los proyectos y las características con que estos se modelan, el lado izquierdo de la limitación puede estar conformado por uno (Kim et al., 2013; Sanci \& Daskin, 2019) o más términos (Meng \& Wang, 2011).

Ambas formas de modelar la limitación de financiación pueden ser reformuladas en el marco de toma de decisiones multi-periodo, estando sujetos habitualmente los proyectos seleccionados en cada periodo al presupuesto disponible del respectivo periodo (Shishebori et al., 2016). Sin embargo, la condición a largo plazo del problema ha permitido ajustar la mencionada limitación a ciertas cuestiones de la realidad (Fattahi et al., 2015), entre estas se encuentra: trasladar el presupuesto remanente al periodo siguiente (Bhattarai et al., 2020), calcular los beneficios marginales de los proyectos con limitación de presupuesto acumulativa (Shayanfar et al., 2016) o contemplar la incertidumbre en la disponibilidad de los recursos (Liu \& Kwon, 2020).

En el MPLEM mono-periodo que se propone en la sección 5.3. se modela la limitación de financiación teniendo en cuenta que, la sumatoria de los costes de inversión de los proyectos que se seleccionan para diseñar la red intermodal sea inferior o igual al presupuesto disponible.

\footnotetext{
${ }^{34}$ Aunque el MPLEM de este capítulo se define en un marco de toma de decisiones mono-periodo, la sección se extiende sutilmente a la toma de decisiones multi-periodo para evitar duplicar la información en los siguientes capítulos.
} 


\subsection{Propuesta del MPLEM mono-periodo con enfoque económico}

En esta sección se formula el MPLEM mono-periodo con enfoque económico que da respuesta al problema combinado LI-DR intermodal descrito en el capítulo 3. En el modelo se incorporan las oportunidades identificadas en la literatura sobre la capacidad en las instalaciones y la limitación de financiación. En la subsección 5.3.1. se enuncian los supuestos básicos del modelo, en la subsección 5.3.2. se indica la notación utilizada en la formulación matemática del modelo y en la subsección 5.3.3. se proporcionan las relaciones matemáticas que formulan el MPLEM mono-periodo.

\subsubsection{Supuestos básicos del MPLEM mono-periodo}

Los supuestos básicos definidos para construir el modelo matemático son los siguientes:

i. Se conocen las localizaciones potenciales de ambos tipos de proyectos (infraestructura de transporte de primera milla y terminales intermodales) y se proporcionan las localizaciones de los nodos de origen, los nodos de destino y la estructura de las redes de transporte subyacentes para los modos por carretera, fluvial y ferroviario.

ii. Los proyectos de primera milla se establecen como arcos unidireccionales con el propósito de determinar las decisiones de flujo desde los nodos de origen. Se considera al menos un potencial proyecto de primera milla para cada nodo de origen. Estos pueden conectar con las redes de transporte subyacente por carretera o fluvial.

iii. Los proyectos de terminales intermodales se introducen como arcos unidireccionales que conectan dos nodos modales diferentes, en los cuales se efectúan actividades de consolidación y transbordo de la carga. Se consideran varias terminales intermodales distribuidas en distintos puntos de la zona de estudio. Conforme con las conexiones de las redes subyacentes, las terminales intermodales se pueden configurar por los modos de transporte carretera-fluvial, carretera-ferroviaria y fluvial-ferroviaria.

iv. Para decidir la localización de cada proyecto se define un conjunto de alternativas de capacidad para los arcos intermodales y un conjunto de alternativas viales para los arcos de primera milla. Las alternativas que corresponde a un mismo proyecto conectan el mismo par de nodos modales. Cada alternativa tiene asociada costes de construcción y, según sea el tipo de proyecto, costes unitarios de transporte u operativos. Los costes unitarios capturan los efectos de la economía de escala y limitan la asignación del flujo.

v. Para localizar un proyecto se debe elegir una única alternativa del conjunto dado, tal y como se muestra en la Figura 5.1. Estas alternativas son de capacidad (arcos intermodales) y viales (arcos de primera milla). Las alternativas tienen implícito un orden para denotar mejores características respecto a la alternativa que le precede, por lo que la alternativa seleccionada para el proyecto $\mathrm{B}$ respecto al proyecto A tiene menores costes operativos unitarios pero mayores costes de construcción. Además, si el proyecto es de tipo intermodal tiene mayor capacidad de consolidación.

vi. Los arcos de las redes de transporte subyacentes pueden ser unidireccionales o bidireccionales. Los arcos bidireccionales se modelan como dos arcos diferentes con el mismo coste unitario de transporte y conexión del mismo par de nodos. Los costes unitarios difieren del modo de transporte. Todas las conexiones son factibles de uso para asignar el flujo y satisfacer la demanda. 
vii. Todos los proyectos de inversión tienen efectos positivos para el acceso de bienes y servicios, tanto como en la movilidad y generación de empleo para la población ubicada en la zona de estudio.

viii. Cada proyecto cumple con los estudios y avales medioambientales para su evaluación y puesta en marcha en la configuración de la red intermodal.

ix. Los proyectos seleccionados para diseñar la red intermodal están sujetos a un presupuesto de inversión, para ello se contempla un único presupuesto de inversión general.

Figura 5.1. Modelado mono-periodo de las alternativas de capacidad/vial de los proyectos de inversión

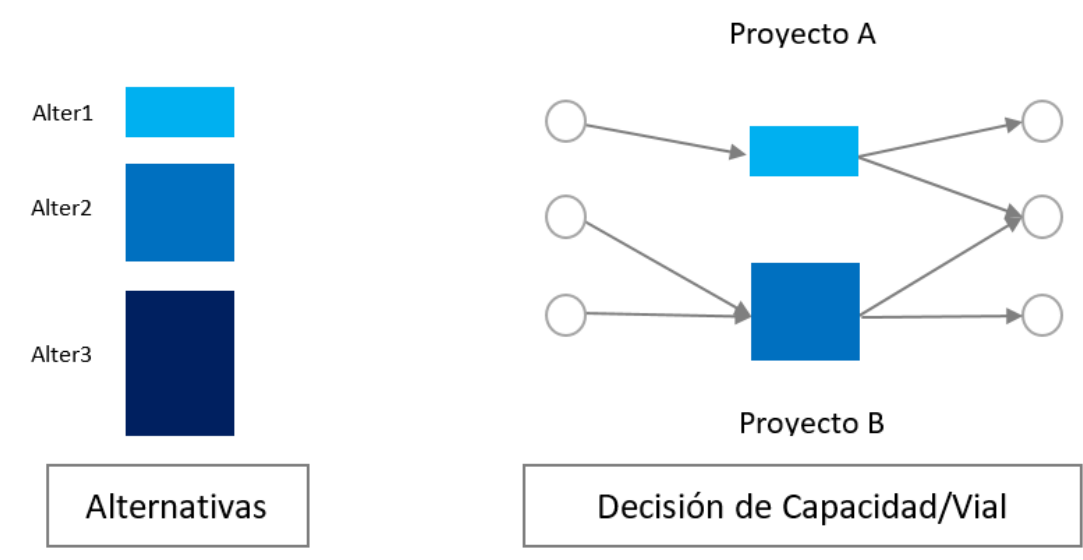

Fuente: Elaboración propia

\subsubsection{Notación del MPLEM mono-periodo}

Para definir la notación matemática del MPLEM mono-periodo con enfoque económico se identifican, a partir de la descripción presentada en la sección 3.4., los conjuntos, subconjuntos, parámetros y variables de decisión. Los conjuntos $I, J$ y $S$ corresponden a los nodos de origen, nodos de destino y nodos modales de las redes de transporte subyacentes, respectivamente. El conjunto $E$ corresponde a los arcos modales de las redes de transporte subyacentes. Los subconjuntos $S_{C}$ y $E_{C}$ corresponden a los nodos y arcos de la red de carretera, $S_{H}$ y $E_{H}$ corresponden a la red fluvial y, $S_{F}$ y $E_{F}$ corresponden a la red ferroviaria. Cada $S \in S$ representa la intersección de al menos dos arcos de una misma red modal. A partir de los conjuntos y subconjuntos identificados se define un grafo dirigido $G=(N, A)$ donde $N$ son los nodos $i \in$ $I, j \in J$ y $s \in S$; y $A$ son los pares $(s, e)$ y $(e, s)$ de las redes de transporte subyacentes, los proyectos potenciales de primera milla y las terminales intermodales. $A$ se conforma por los subconjuntos disjuntos $A=A_{1} \cup A_{2} \cup A_{3} \cup A_{4} \cup A_{5}$.

Los subconjuntos de $\operatorname{arcos} A_{1}$ y $A_{2}$ conectan los nodos de origen $I$ con los nodos de las redes modales $S$. El subconjunto $A_{1}$ representa los potenciales proyectos de primera milla y $A_{2}$ las antiguas vías de primera milla. El subconjunto de $\operatorname{arcos} A_{3}$ agrupa los pares $(s, e)$ y $(e, s)$ de las redes de transporte subyacentes. El subconjunto de $\operatorname{arcos} A_{4}$ representa las potenciales terminales intermodales que conectan dos nodos modales diferentes, por ejemplo $s_{C} \rightarrow s_{H}$, $s_{C} \rightarrow s_{F}$ y $s_{H} \rightarrow s_{F}$. El subconjunto de $\operatorname{arcos} A_{5}$ constituye los arcos finales que conectan los 
nodos de las redes de transporte $S$ con los nodos de destino $J$. Las alternativas viales de los $\operatorname{arcos} A_{1}$ y las alternativas de capacidad de $\operatorname{los} \operatorname{arcos} A_{4}$ se representan por el conjunto $K$. Las matrices de conexión $\varphi$ y $\alpha$ se suministran para los respectivos flujos de entrada y salida en cada arco del grafo.

En concordancia con el objetivo del modelo se suministran los costes de transporte $C t t e_{k, a}$ y $C t t e_{a}$, y los costes operativos $\operatorname{Coper}_{k, a}$. El resto de los parámetros y las variables de decisión utilizados para formular el MPLEM mono-periodo con enfoque económico se detallan en la Tabla 5.1.

Tabla 5.1. Notación utilizada en el MPLEM mono-periodo

\begin{tabular}{ll}
\hline Conjuntos y subconjuntos \\
\hline$I$ & Conjunto de nodos de origen \\
$J$ & Conjunto de nodos de destino \\
$S$ & Conjunto de nodos de las redes de transporte subyacentes \\
$S_{C}$ & Subconjunto de nodos de la red de carreteras \\
$S_{H}$ & Subconjunto de nodos de la red fluvial \\
$S_{F}$ & Subconjunto de nodos de la red ferroviaria \\
$E$ & Conjunto de arcos de las redes de transporte subyacentes \\
$E_{C}$ & Subconjunto de arcos de la red de carreteras \\
$E_{H}$ & Subconjunto de arcos de la red fluvial \\
$E_{F}$ & Subconjunto de arcos de la red ferroviaria \\
$A$ & Conjunto de arcos de la red de entrada $A=A_{1} \cup A_{2} \cup A_{3} \cup A_{4} \cup A_{5}$ \\
$A_{1}$ & Subconjunto de arcos que representan los proyectos de primera milla \\
$A_{2}$ & Subconjunto de arcos que representan las antiguas vías de primera milla \\
$A_{3}$ & Subconjunto de arcos que representan los pares $(s, e)$ y $(e, s)$ \\
$A_{4}$ & Subconjunto de arcos que representan los proyectos de terminales intermodales \\
$A_{5}$ & Subconjunto de arcos que representan los arcos finales \\
$K$ & Conjunto de alternativas empleadas para localizar los dos tipos de proyectos de inversión
\end{tabular}

\section{Parámetros}

$P_{i} \quad$ Cantidad de producto disponible en el nodo de origen $i \in I$

$D_{j} \quad$ Cantidad de producto requerido en el nodo de destino $j \in J$

Ctte $_{k, a}$ Coste unitario de transporte para la alternativa $k \in K$ del arco $a \in A_{1}$

Ctte $_{a}$ Coste unitario de transporte del arco $a \in A_{2} \cup A_{3} \cup A_{5}$

Coper $_{k, a}$ Coste unitario operativo para la alternativa $k \in K \operatorname{del} \operatorname{arco} a \in A_{4}$

$\operatorname{Ccon}_{k, a}$ Coste de construir la alternativa $k \in K$ del arco $a \in A_{1} \cup A_{4}$

$\operatorname{Cap}_{k, a}$ Capacidad de la alternativa $k \in K \operatorname{del} \operatorname{arco} a \in A_{4}$ 


\begin{tabular}{ll}
\hline PInv & Presupuesto de inversión disponible \\
$M$ & Número grande utilizado para ubicar la alternativa $k \in K$ del $\operatorname{arco} A_{1}$ \\
$\varphi_{a}$ & Matriz de conexión para los flujos de entrada del arco $a \in A$ \\
$\alpha_{a}$ & Matriz de conexión para los flujos de salida del arco $a \in A$ \\
\hline Variables de decisión & \\
\hline$\beta_{k, a}$ & Toma el valor de 1 si se construye la alternativa $k \in K$ del arco $a \in A_{1} \cup A_{4} ; 0$ en caso contrario \\
$\widehat{X}_{k, a}$ & Cantidad de producto que fluye por la alternativa $k \in K$ del arco $a \in A_{1} \cup A_{4}$ \\
$\breve{X}_{a}$ & Cantidad de producto que fluye por el arco $a \in A_{2} \cup A_{3} \cup A_{5}$ \\
$W_{i}$ & Cantidad de producto que se pierde en el nodo de origen $i \in I$ \\
\hline
\end{tabular}

\subsubsection{Formulación del MPLEM mono-periodo}

Para construir el MPLEM mono-periodo con enfoque económico se toma como referencia la formulación de Amaral et al. (2012) presentada en la sección 3.4. El modelo que se desarrolla para este capítulo minimiza los costes de transporte y operativos de la red intermodal que se diseña. El MPLEM permite seleccionar los proyectos de primera milla y terminales intermodales que reducen tales costes, al mismo tiempo que decide las capacidades con la que operan las terminales intermodales, las características de las vías de primera milla y los flujos en cada arco modal de la red. La formulación del modelo matemático es la siguiente:

$$
\min \sum_{k \in K} \sum_{a \in A_{1}} \text { Ctte }_{k, a} \cdot \hat{X}_{k, a}+\sum_{a \in A_{2} \cup A_{3} \cup A_{5}} \text { Ctte }_{a} \cdot \breve{X}_{a}+\sum_{k \in K} \sum_{a \in A_{4}} \operatorname{Coper}_{k, a} \cdot \hat{X}_{k, a}
$$

Sujeto a:

$$
\begin{array}{cr}
\sum_{k \in K} \sum_{a \in A_{1}} \varphi_{a} \cdot \hat{X}_{k, a}+\sum_{a \in A_{2}} \varphi_{a} \cdot \breve{X}_{a}+W_{i}=P_{i} & \forall i \in I \\
\sum_{a \in A_{5}} \alpha_{a} \cdot \breve{X}_{a}=D_{j} & \forall j \in J \\
\varphi_{a} \cdot \breve{X}_{a \mid(e, s)}+\varphi_{a} \cdot \hat{X}_{k, a \mid(e, s)}=\alpha_{a} \cdot \breve{X}_{a \mid(s, e)}+\alpha_{a} \cdot \hat{X}_{k, a \mid(s, e)} & \forall a \in A ; k \in K \\
\hat{X}_{k, a} \leq M \cdot \beta_{k, a} & \forall k \in K ; a \in A_{1} \\
\hat{X}_{k, a} \leq \operatorname{Cap}_{k, a} \cdot \beta_{k, a} & \forall k \in K ; a \in A_{4} \\
\sum_{k \in k} \beta_{k, a} \leq 1 & \forall a \in A_{1} \cup A_{4} \\
\sum_{k \in K} \sum_{a \in A_{1} \cup A_{4}} \operatorname{Ccon}_{k, a} \cdot \beta_{k, a} \leq P \operatorname{PInv} & \forall t \in T
\end{array}
$$




$$
\begin{array}{cc}
\beta_{k, a} \in\{0,1\} & \forall k \in K ; a \in A_{1} \cup A_{4} \\
\hat{X}_{k, a} \geq 0 & \forall k \in K ; a \in A_{1} \cup A_{4} \\
\breve{X}_{a} \geq 0 & \forall a \in A_{2} \cup A_{3} \cup A_{5}
\end{array}
$$

La función objetivo (5.1) minimiza los costes de uso de la red intermodal que se diseña. El primer término calcula los costes de transporte para el flujo asignado por la alternativa vial seleccionada para cada nuevo arco modal de primera milla que se localiza, el segundo término calcula los costes de transporte para el flujo asignado a los arcos modales de las redes de transporte subyacentes, y el tercer término calcula los costes operativos para el flujo asignado por la alternativa de capacidad seleccionada en cada terminal intermodal que se localiza.

Las restricciones (5.2) establecen que el producto asignado desde un nodo de origen a través de las nuevas y antiguas vías de primera milla y el producto que se pierde sean equivalentes a la cantidad de producto disponible en el respectivo nodo de origen. Las restricciones (5.3) determinan que la cantidad de producto que se demanda en cada nodo de destino debe ser satisfecho en su totalidad. Las restricciones (5.4) establecen que el flujo en cada arco de la red debe estar balanceado. Las restricciones (5.5), (5.6) y (5.7) aseguran que el flujo asignado a los arcos de primera milla y a los arcos intermodales se efectúe de acuerdo con la alternativa vial y la alternativa de capacidad que se decida al localizar el arco. La alternativa de capacidad seleccionada limita el flujo asignado a los arcos intermodales. Las restricciones (5.8) garantizan que los costes de construcción de todas las alternativas seleccionadas para diseñar la red intermodal no excedan el presupuesto de inversión disponible. El conjunto de restricciones (5.9), (5.10) y (5.11) definen los dominios de las variables de decisión.

\subsection{Análisis de la solución óptima}

Para analizar el comportamiento de la solución de DERTI que se obtiene al resolver el MPLEM mono-periodo, se proporcionan distintos valores a los parámetros de demanda, capacidades de las alternativas de los arcos intermodales y presupuesto de inversión. En la Tabla 5.2. se detallan los valores definidos para estos parámetros. A partir de los valores descritos se generaron 600 instancias al modelo y se resolvieron aplicando el esquema de resolución R+Gurobi, expuesto en la subsección 3.5.1. Es importante mencionar que, las capacidades ajustadas (Cap1) equivalen al 40\% de las capacidades amplias (Cap2) y el presupuesto de inversión P100 equivale al 100\% de la suma total del coste de inversión de todos los proyectos potenciales. La suma total de los costes de construcción con Cap1 corresponden al $79.21 \%$ de la suma total de Cap2.

Tabla 5.2. Valores de los parámetros del MPLEM mono-periodo

\section{Valores}

\begin{tabular}{ll}
\hline$D_{j}$ & Actual (Dem1), $+20 \%($ Dem2), -10\% (Dem3) \\
$K$ & Ajustadas (Cap1), Amplias (Cap2)
\end{tabular}


$\operatorname{Cap}_{k, a} 0.33$ (Alter1), 0.66 (Alter2), 1 (Alter3)

PInv $\quad 1 \%(\mathrm{P} 1), 2 \%(\mathrm{P} 2), \ldots, 100 \%(\mathrm{P} 100)$

En las siguientes subsecciones se informan los análisis realizados. En la subsección 5.4.1. se analiza el comportamiento de la solución respecto a los costes de uso de la red intermodal, en la subsección 5.4.2. respecto a las decisiones de localización y capacidad del DERTI y en la subsección 5.4.3. respecto a las decisiones de asignación de los flujos del DERTI. Los análisis revelan un importante efecto provocado por el excedente de producto en los nodos de origen, por lo tanto, se valida la formulación incorporando una nueva restricción al modelo que compense este efecto. Los resultados de la validación se presentan en la subsección 5.4.4.

\subsubsection{Análisis de los costes de uso de la red intermodal}

Las Figuras 5.2. y 5.3. muestran las soluciones óptimas en los costes de uso de la red para los distintos presupuestos de inversión y niveles de demanda. Las curvas son construidas a partir de los resultados obtenidos de cada instancia resuelta. Los porcentajes que se muestran en cada curva representan la proporción de los costes frente al coste obtenido con el presupuesto factible más bajo para ese nivel de demanda, por tanto, las comparaciones entre los niveles de demanda no son relativas. Los espacios sombreados indican el intervalo de presupuestos en el que no es factible obtener una solución óptima, y a su vez determinan la mínima inversión requerida para localizar al menos un proyecto que conecte dos redes modales de transporte para asignar los flujos. Las líneas punteadas indican el presupuesto de inversión necesario para diseñar la red intermodal con los costes más bajo. Las líneas grises indican los costes equivalentes en los otros niveles de demanda para el respectivo nivel de presupuesto.

Por ejemplo, para Cap1 con Dem1 no se obtienen soluciones factibles con disponibilidades de presupuestos entre P1 y P9, por lo que la mínima inversión necesaria es de P10. Por su parte, de todas las soluciones óptimas, los costes más bajos (70.64\%) se obtienen con una disponibilidad de P60. Con recursos económicos superiores a esta disponibilidad se puede expandir la red intermodal, pero las decisiones no tendrían efectos en los costes. Sin embargo, la disponibilidad de P60 frente a variaciones de la demanda impacta en distintas direcciones. Con Dem2 se lograría absorber la mayoría de los efectos ya que la solución óptima con el coste más bajo se ubica en P63. Los tomadores de decisiones podrán valorar, ante un posible incremento de la demanda, si es conveniente asumir un 3\% más de inversión para mejorar en $0.05 \%$ los costes de uso de la red. Por el contrario, con Dem3 el coste más bajo se ubica en $\mathrm{P} 49$. Esto puede ocasionar que algunos de los proyectos localizados queden subutilizados.

Con Cap2, aunque los resultados son similares a los mencionados para Cap1, se presentan algunas diferencias significativas. La inversión mínima factible no cambia (P10), mientras que, el requerimiento máximo sí. Para Dem1 es P59, Dem2 es P61 y Dem3 es P51. Los costes de uso de la red son más sensibles ante variaciones en el presupuesto a medida que se reduce la disponibilidad de inversión. La solución con el coste más bajo tiene efectos más pronunciados en cada nivel de demanda, sustancialmente en Dem1 que se reduce hasta el $66.67 \%$. Los cambios en la demanda se capturan mejor siempre que la disponibilidad de inversión atienda al máximo requerimiento, con ello el margen de compensación de los proyectos subutilizados también se reduce. Los tomadores de decisiones podrán considerar situarse cerca de P51 y 
asumir los costes ante un posible incremento de la demanda. Los incrementos no exceden de $0.03 \%$ para Dem1 y $1.01 \%$ para Dem2.

Figura 5.2. Soluciones óptimas en los costes de uso de la red con Cap1

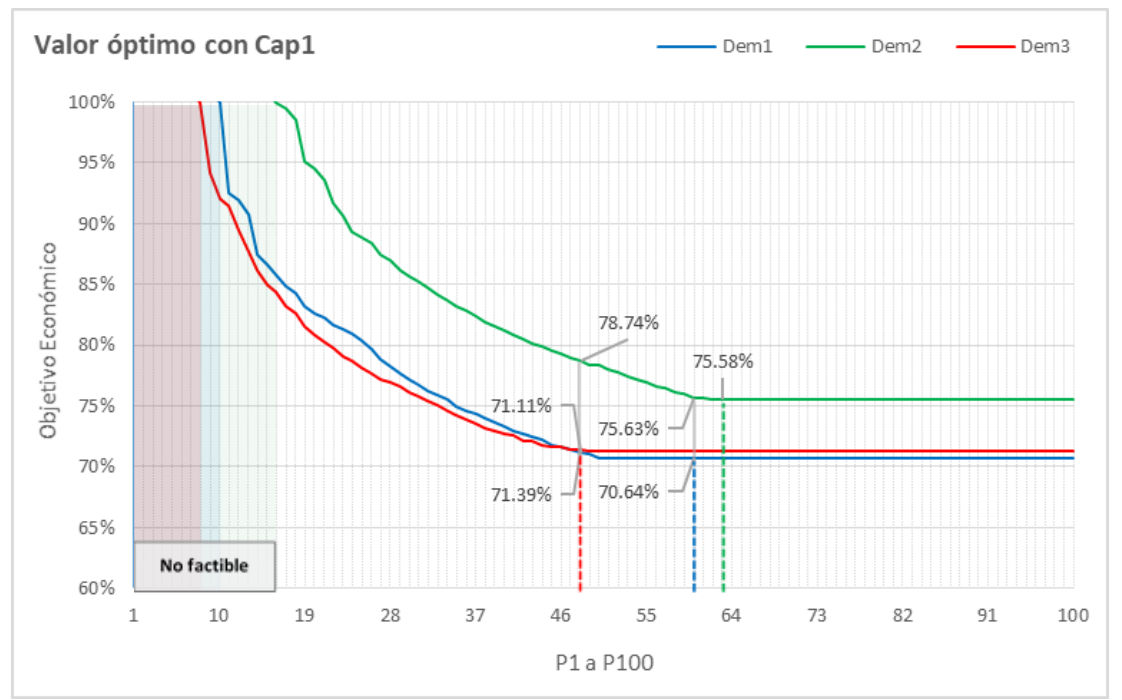

Fuente: Elaboración propia

Figura 5.3. Soluciones óptimas en los costes de uso de la red con Cap2

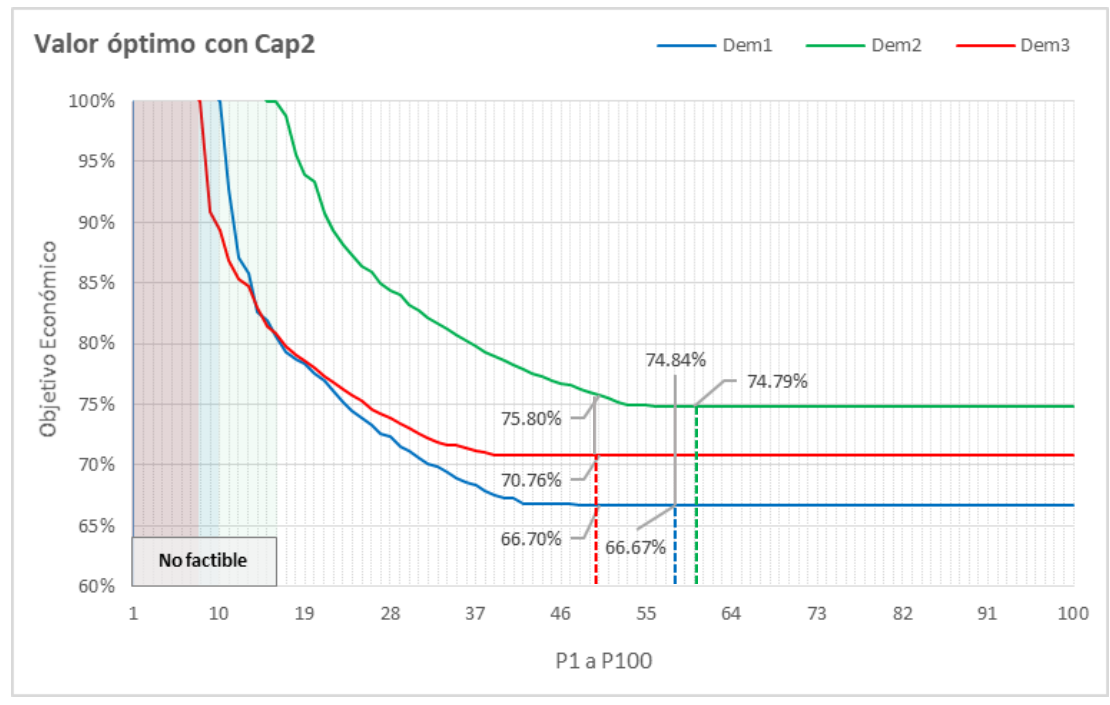

Fuente: Elaboración propia

\subsubsection{Sensibilidad en el DERTI: decisiones estratégicas}

La Figura 5.4. contrasta las soluciones óptimas de DERTI obtenidas con Cap1 y Cap2 al resolver el modelo para Dem1 y presupuestos P10, P25, P35 y P50. Las soluciones representan las decisiones estratégicas de localización y capacidad de las instalaciones intermodales, la localización y alternativa vial de los arcos de primera milla, y los arcos de las redes subyacentes seleccionados para asignar los flujos. En principio se podría creer que con un reducido 
presupuesto se identifican decisiones básicas en el diseño de la red y a medida que se incrementa la disponibilidad se expande la red, aunque los dos tipos de capacidades no muestran en su totalidad este comportamiento. En general, se puede decir que, con bajas disponibilidades se decide primero la localización de terminales intermodales, las características de la red mejoran cuando el presupuesto es mayor, y el tipo de capacidad ejerce una gran influencia en el diseño intermodal de la red.

El efecto de la disponibilidad del presupuesto de inversión en los diseños con Cap1 revela que las decisiones de localización son menos sensibles que las decisiones de capacidad de la terminal y de alternativa vial de primera milla. En estos se prefiere la localización del mayor número de terminales intermodales debido a la reducida cantidad de producto que se puede consolidar en ellas para aprovechar las economías de escala, por lo que se apoya principalmente en la red de carreteras para satisfacer la demanda. En estos diseños se tiende a excluir un nodo de origen para satisfacer la demanda. Por su parte, como Cap2 permite mayor consolidación de producto, en los diseños se percibe la concentración de los flujos intermodales en solo 2 o 3 terminales cuando el presupuesto tiende al mínimo requerimiento. Con más disponibilidad se favorecen las decisiones de localización de proyectos de primera milla, se acentúa el uso de la red fluvial y ferroviaria para satisfacer la demanda, e inclusive, todos los nodos de origen se benefician no solo con la mejora de la infraestructura de primera milla sino también con la participación en el mercado.

Los diseños de la Figura dan cuenta de la estructura intermodal resultante, así como de la importancia en la incorporación de las redes subyacentes para dar solución al problema. Pese a que las decisiones estratégicas de localización y capacidad y las decisiones tácticas de asignación son perceptibles, con esta representación no se puede realizar un análisis detallado sobre el aprovechamiento de la capacidad instalada o sobre la posibilidad de desestimar alguna decisión de inversión. Por ende, en la siguiente subsección se profundiza en estos aspectos, considerando el uso de la red a partir de la asignación de los flujos. 
Figura 5.4. Decisiones estratégicas en las soluciones de DERTI

\section{Soluciones DERTI con Dem1 y P10}

PInv

PO

P10

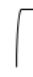

Cap1

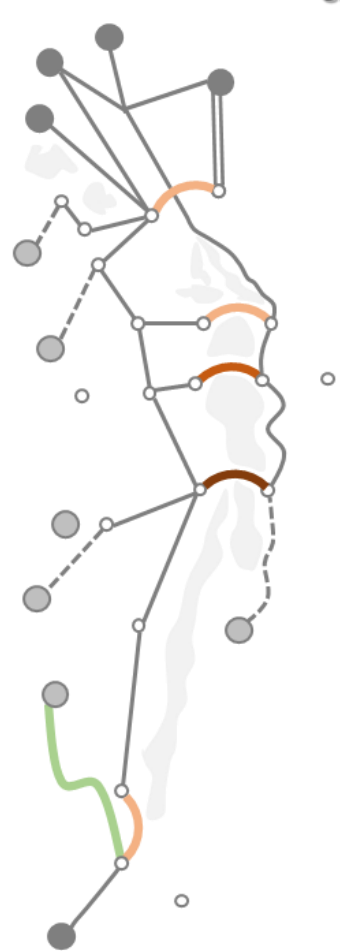

\section{Cap2}
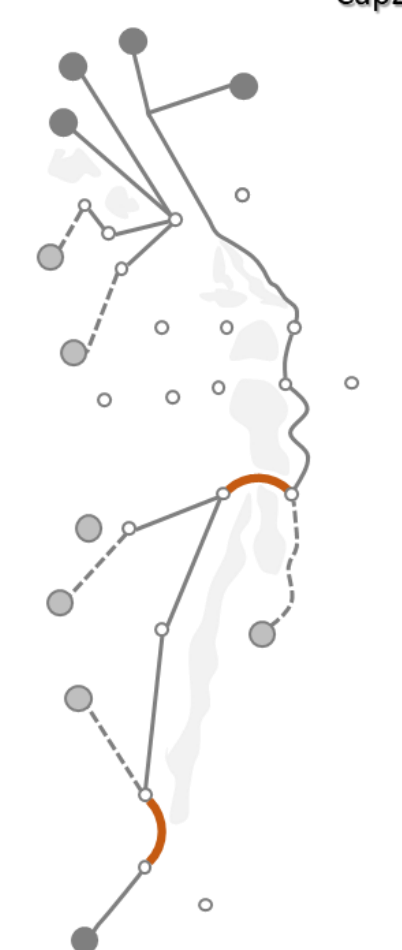

b.

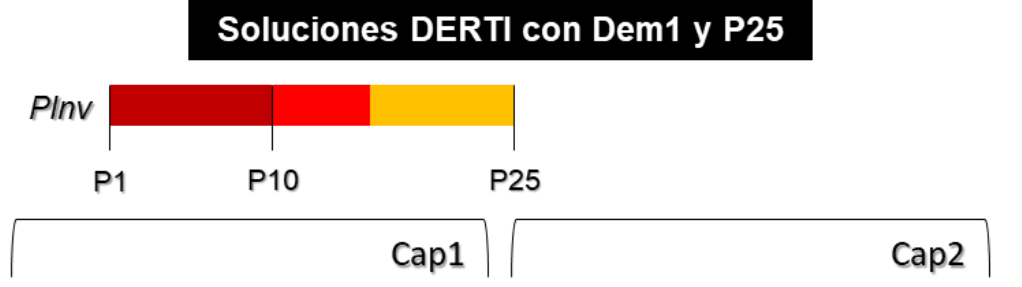


c.

Soluciones DERTI con Dem1 y P35

PInv
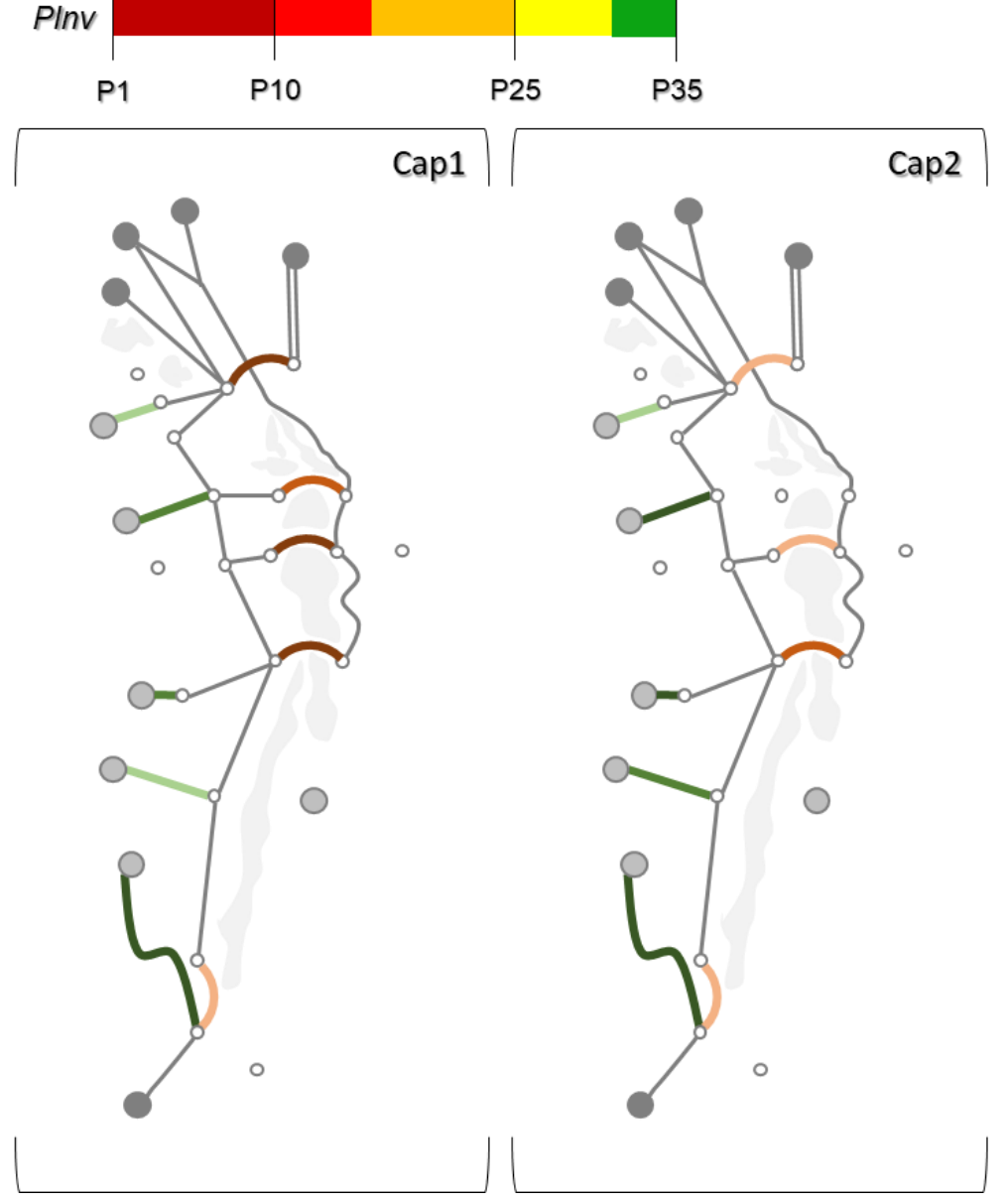

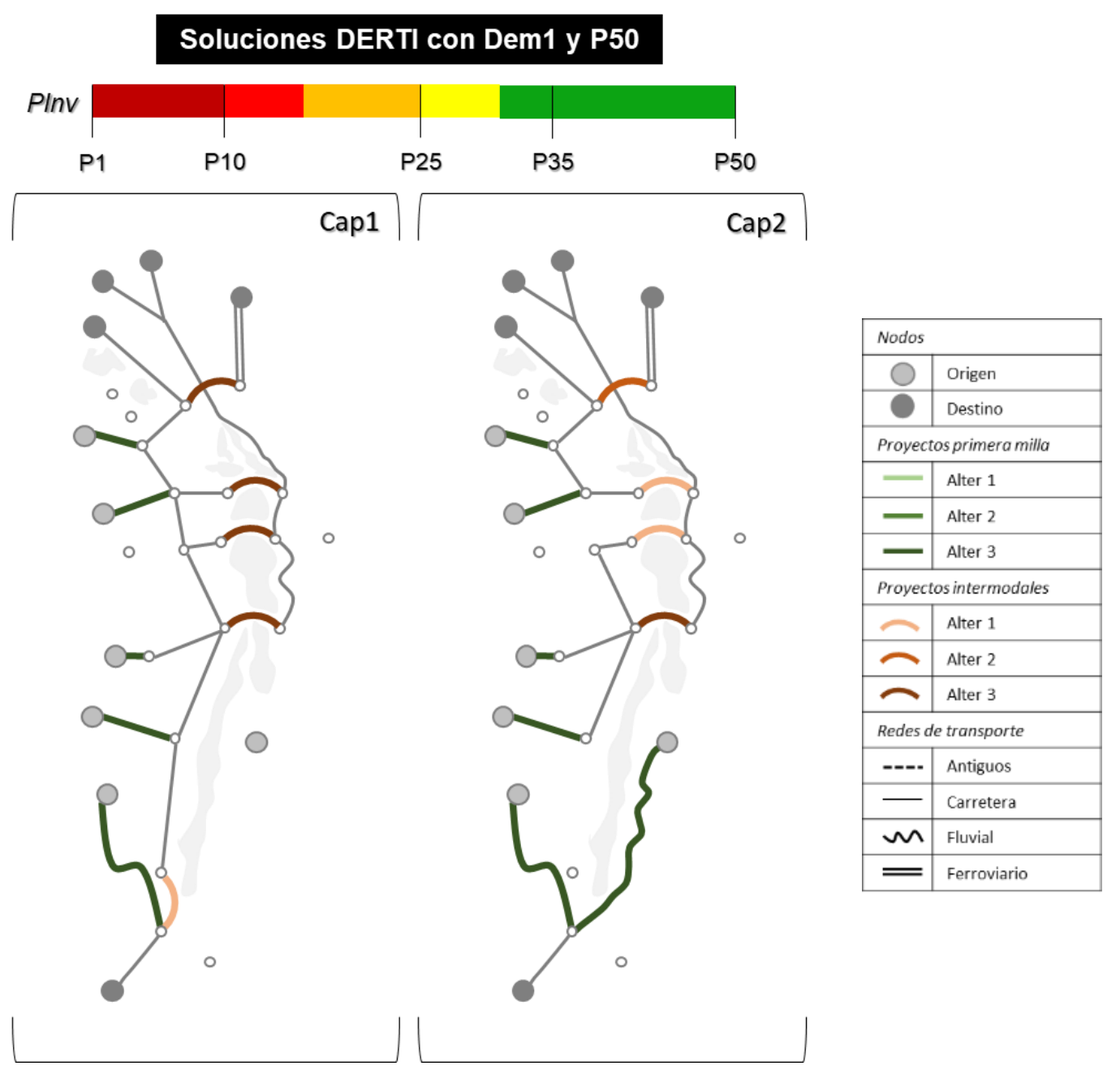

Fuente: Elaboración propia 


\subsubsection{Sensibilidad en el DERTI: decisiones tácticas}

El problema combinado LI-DR intermodal está integrado por decisiones estratégicas y tácticas con reciproca influencia en la toma de decisiones, por lo tanto, las decisiones tácticas en la solución óptima de DERTI también se ve comprometida ante las variaciones a los valores de los parámetros. En esta sección se informa el análisis en este sentido contemplando el uso de la capacidad intermodal instalada y el uso de las redes de transporte subyacente.

\subsubsection{1. $\quad$ Uso de la capacidad intermodal de las instalaciones}

La Figura 5.5. muestra, para distintas disponibilidades de inversión y el nivel de demanda Dem1, el porcentaje de uso de la capacidad instalada respecto a la decisión de localización y alternativa de capacidad de cada proyecto intermodal evaluado en la red ${ }^{35}$. Para comprender los cambios en estas decisiones es importante tener en cuenta los supuestos básicos iv y v expuestos en la subsección 5.3.1., en relación con los costes unitarios y la selección de las alternativas. Los números del 1 al 7 corresponden a los proyectos intermodales, la configuración modal de estos es la siguiente: del 1 a 4 tienen la configuración carretera-fluvial, el 5 es carretera-ferroviario y el 6 y 7 son fluvial-ferroviario. Los colores diferencian las alternativas de capacidad y los matices responden al porcentaje de uso de la misma a partir de los flujos asignados.

Con Cap1, a medida que se incrementa el presupuesto disponible, se selecciona la alternativa con mejores características de consolidación de producto, con el propósito de aprovechar los beneficios que se generan en la reducción de los costes operativos. El distintivo de "ajustadas" de Cap1 conlleva a que cinco de los siete proyectos sean considerados vitales para configurar la red. Los proyectos intermodales seleccionados tienden al máximo uso de la capacidad, con excepción del proyecto 4 cuyo uso no supera el 50\%, y para disponibilidades superiores a P55 no es factible su elección. Por su parte, las alternativas de Cap2 distinguidas por ser "amplias" consolidan mayor cantidad de producto y flexibilizan la selección del proyecto y la alternativa para configurar la red. Estas revelan con claridad la dimensión e importancia de las terminales intermodales en la red, en otras palabras, se identifican proyectos de alta envergadura y primordiales como lo es el proyecto 3, y otros de mediana y menor envergadura como son los proyectos 5 y 2 . De igual manera que con Cap1 el proyecto 4 implica un reducido uso de la capacidad, en este caso no supera el $25 \%$ de la capacidad instalada.

Comparando las decisiones resultantes se hace manifiesta la necesidad de valorar, por parte de los tomadores de decisión, la opción de garantizar y asumir la inversión en algunas terminales que no hacen uso eficiente de la capacidad instalada, como en el proyecto 4. Al consultar los resultados de flujos asignados específicamente para este proyecto se observa que con Cap2 el uso de la capacidad no supera el 10\% cuando el presupuesto disponible está entre P20 y P45, asimismo, para el proyecto 1 el valor no excede de $35 \%$ para presupuestos mayores a P45. No obstante, para solventar estos efectos es conveniente considerar, en una futura extensión del MPLEM, una limitación relacionada con la capacidad mínima de uso para

\footnotetext{
${ }^{35}$ Es preciso señalar que el presupuesto de inversión también se agota localizando las alternativas de los proyectos de primera milla. Las decisiones relacionadas con este tipo de proyecto no se incluyen en la Figura.
} 
seleccionar la alternativa del proyecto. Esta limitación, seguramente, alteraría el DERTI a nivel estratégico y táctico, por lo que es posible complementar a futuro el análisis en este sentido.

Figura 5.5. Uso de la capacidad instalada en las terminales intermodales con Dem1

\begin{tabular}{|c|c|c|c|c|c|c|c|c|c|c|c|c|c|c|c|c|}
\hline \multirow{2}{*}{ PInv } & \multicolumn{7}{|c|}{ Cap1 } & \multicolumn{7}{|c|}{ Cap2 } & \multirow{3}{*}{$\frac{\vec{y}}{\frac{\vec{U}}{\alpha}}$} & \multirow{2}{*}{$\begin{array}{l}0.01-0.249 \\
0.25-0.499\end{array}$} \\
\hline & 1 & 2 & 3 & 4 & 5 & 6 & 7 & 1 & 2 & 3 & 4 & 5 & 6 & 7 & & \\
\hline P10 & & & & & & & & & & & & & & & & $0.50-0.149$ \\
\hline P15 & & & & & & & & & & & & & & & & \\
\hline P20 & & & & & & & & & & & & & & & & $0.01-0.249$ \\
\hline P25 & & & & & & & & & & & & & & & & $0.25-0.499$ \\
\hline P30 & & & & & & & & & & & & & & & $\frac{U^{2}}{\alpha}$ & $0.50-0.749$ \\
\hline P35 & & & & & & & & & & & & & & & & $0.75-1.0$ \\
\hline P40 & & & & & & & & & & & & & & & & \\
\hline P45 & & & & & & & & & & & & & & & & $0.01-0.249$ \\
\hline P50 & & & & & & & & & & & & & & & 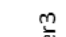 & $0.25-0.499$ \\
\hline P55 & & & & & & & & & & & & & & & $\frac{\pi}{4}$ & $0.50-0.749$ \\
\hline $\mathrm{P} 60$ & & & & & & & & & & & & & & & & $0.75-1.0$ \\
\hline
\end{tabular}

Fuente: Elaboración propia

En la Figura 5.6. aunque se muestra el porcentaje de uso de la capacidad instalada con Cap2 para los tres niveles de demandas establecidos, parece interesante enfocar el análisis en las semejanzas y diferencias que pueden surgir ante los cambios de demanda al acogerse a un diseño de red especifico. Para todos los niveles de demanda el proyecto 3 es esencial en el diseño y tiende a utilizarse la totalidad de su capacidad. Contrario a lo que podría considerarse para el proyecto 4, pasar del nivel Dem1 a Dem2 anula la viabilidad de seleccionar el proyecto con presupuestos superiores a P25. En tal caso de incrementarse la demanda el proyecto 2 es más atractivo para absorber los nuevos flujos, pero es una decisión incompatible con Dem1 cuando la disponibilidad financiera tiende al menor valor de presupuesto. Una particularidad con Dem2 es la selección del proyecto 7 y la escasa funcionalidad del proyecto 1, siendo este último determinante en la mayoría de los diseños de Dem1 y Dem3. Ante una situación de reducción de la demanda se hace menos necesaria la localización de terminales intermodales, por lo que se deduce que es preferible asignar los recursos económicos a los proyectos de primera milla. 
Figura 5.6. Uso de la capacidad instalada en las terminales intermodales con Cap2

\begin{tabular}{|c|c|c|c|}
\hline \multirow{2}{*}{ PInv } & Dem1 & Dem2 & Dem3 \\
\hline & \begin{tabular}{|l|l|l|l|l|l|l|}
1 & 2 & 3 & 4 & 5 & 6 & 7 \\
\end{tabular} & \begin{tabular}{|l|l|l|l|l|l|l}
1 & 2 & 3 & 4 & 5 & 6 & 7
\end{tabular} & \begin{tabular}{|l|l|l|l|l|l|l}
1 & 2 & 3 & 4 & 5 & 6 & 7 \\
\end{tabular} \\
\hline P10 & & & \\
\hline $\mathrm{P} 15^{*}$ & & & \\
\hline P20 & & & \\
\hline P25 & & & \\
\hline P30 & & & \\
\hline P35 & & & 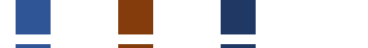 \\
\hline P40 & & & $\square$ \\
\hline P45 & & & 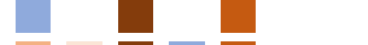 \\
\hline P50** & & 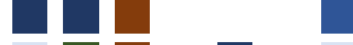 & 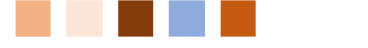 \\
\hline P55 & & & \\
\hline P60 & & & \\
\hline
\end{tabular}

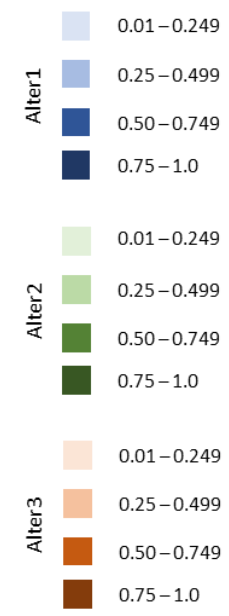

* Para Dem2 la mínima inversión factible para obtener una solución óptima es P15.

** Para Dem3 la máxima inversión requerida es P50.

Fuente: Elaboración propia

\subsubsection{2. $\quad$ Uso de las redes de transporte subyacentes}

La Figura 5.7. representa el porcentaje de demanda asignado a cada arco de la red cuando se considera el nivel de demanda Dem1 y los presupuestos P10 y P25 ${ }^{36}$. Con P10, pese a que las terminales intermodales localizadas están configuradas por los modos carretera-fluvial, el diseño táctico de la red tiene un uso limitado del modo por carretera. Este efecto es entendible puesto que el diseño estratégico resultante no proporciona opciones de mejora en los arcos de primera milla y los movimientos a través de la red se reducen para compensar los altos costes incurridos por hacer uso de los antiguos arcos de primera milla. Mientras, a través de la red fluvial fluye poco más del $40 \%$ de la demanda. Este porcentaje se logra porque esta sección de la red conecta con los arcos finales. Por su parte, Con P25 se estimula el uso de la red de carreteras y se diversifica el uso de la red fluvial. En esta última a través de los arcos finales debido a la localización de una terminal intermodal adicional.

\footnotetext{
${ }^{36}$ Como se menciona al inicio de esta sección, el presupuesto de inversión P10 equivale al $10 \%$ de la suma total del coste de inversión de todos los proyectos potenciales. De esta misma manera para P25.
} 
Figura 5.7. Uso de las redes de transporte subyacentes
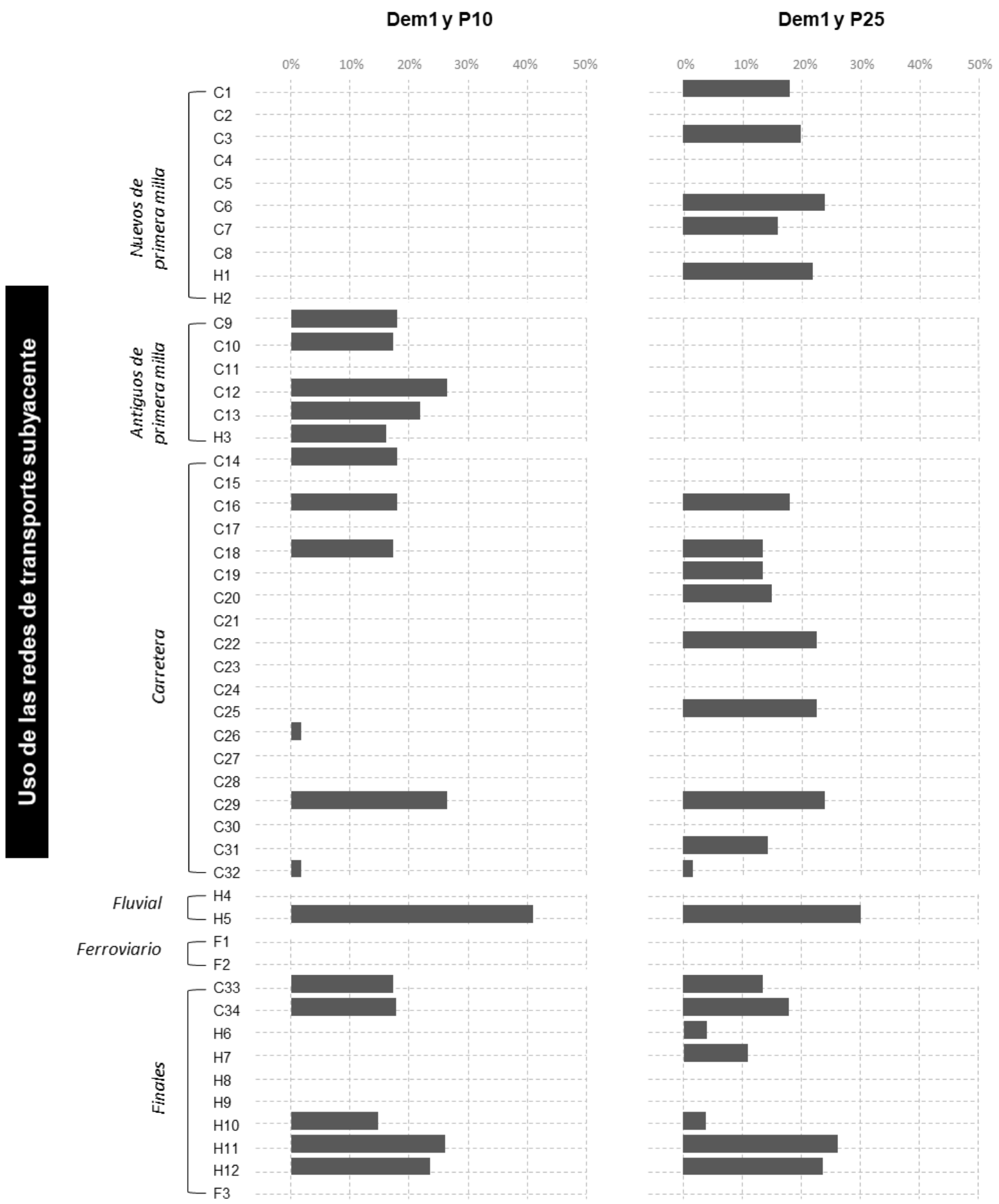

Fuente: Elaboración propia

\subsubsection{Pérdidas de producto en origen}

El análisis a la solución estratégica de DERTI presentado en la subsección 5.4.2. descubren de manera sutil los desequilibrios latentes en la región a causa del excedente de producto que se pierde en los nodos de origen. Seis de los ocho diseños dan cuenta que en un solo nodo puede perderse todo el producto que se oferta, pues, queda excluido del diseño. Para profundizar en estos resultados, las Figuras 5.8. y 5.9. reproducen los porcentajes de producto perdido en cada 
nodo de origen para el nivel de demanda Dem1 y presupuestos P10 a P60. Tanto en las soluciones con Cap1 como con Cap2 los nodos 4 y 6 asumen los mayores porcentajes de pérdidas, siendo más crítica la situación en el nodo 6 pues llega a asumir pérdidas del 100\% del producto ofertado. Aunque no se profundiza en el impacto económico que se ocasiona, Shukla \& Jharkharia (2013) afirman que las pérdidas que asume el agricultor se relacionan directamente con el margen de beneficios. Además, teniendo en cuenta la situación real, es válido considerar que la exclusión de un nodo de origen puede incrementar las desigualdades intrarregionales y, en definitiva, dificultar el desarrollo de la subregión que se estudia.

La premisa anterior se apoya en la expuesto por Witte et al. (2014), quien plantea que la inversión en infraestructura fomenta el crecimiento económico de las regiones, promueve el desarrollo de las actividades económicas, la conexión con nuevos mercados, y el acceso de bienes y servicios a la región (Alstadt et al., 2012). La desvinculación de uno o varios nodos de origen del diseño de la red deteriora la actividad comercial que se ocasiona por la falta de inversión en estas áreas.

Figura 5.8. Porcentaje de pérdidas de producto en los nodos de origen con Cap1

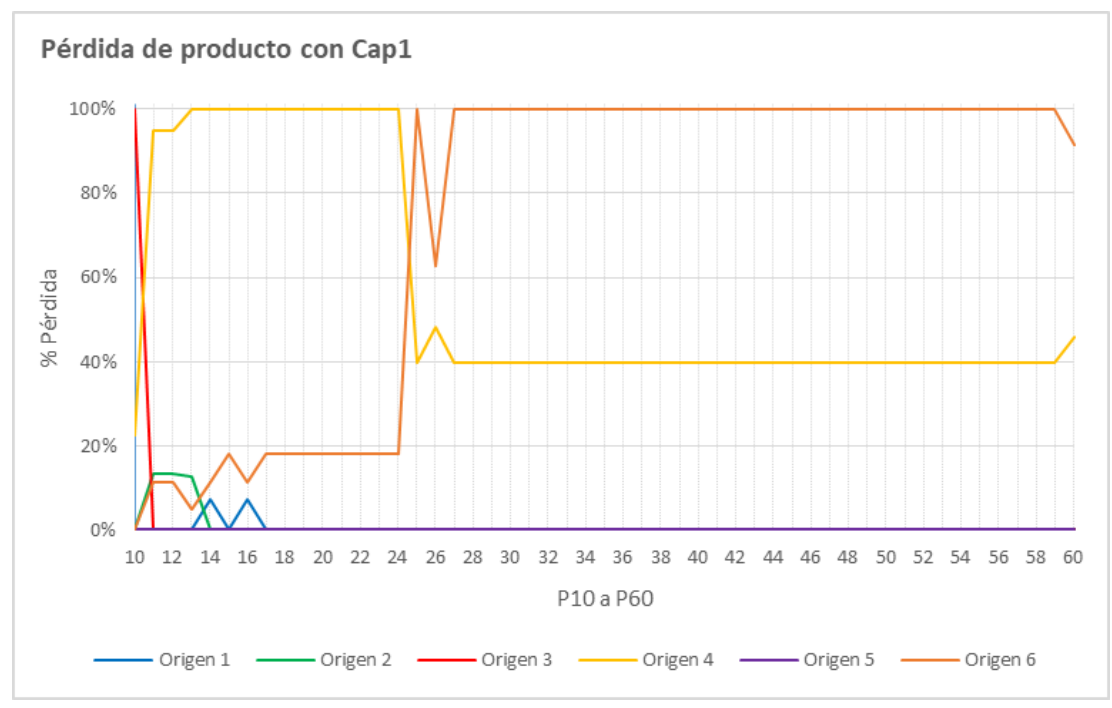

Fuente: Elaboración propia 
Figura 5.9. Porcentaje de pérdidas de producto en los nodos de origen con Cap2

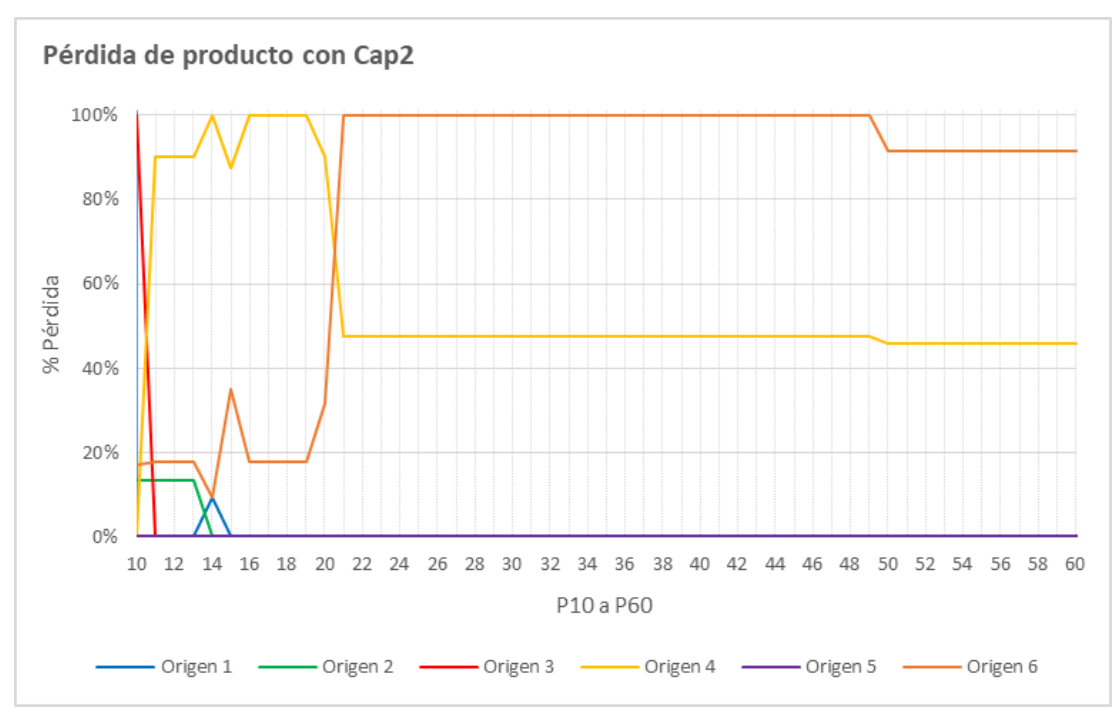

Fuente: Elaboración propia

Para validar los efectos de las pérdidas de producto en el MPLEM mono-periodo se incorpora en la formulación una nueva limitación. Las restricciones (5.12) permiten establecer un límite máximo de pérdidas de producto en cada nodo de origen. El límite se fija calculando la cantidad de producto ofertado que es posible dejar de asignar desde cada nodo de origen. El cálculo se estima definiendo un nuevo parámetro en el modelo con el cual se especifica tal cantidad. El parámetro $\gamma$ se declara como una proporción que se multiplica por la cantidad de producto ofertado. La validación de la nueva restricción se efectúa definiendo dos valores para el parámetro $\gamma=\{0.35,0.50\}^{37}$ y ejecutando 4 instancias del modelo matemático (5.1) a (5.12) para una red de entrada con Dem1 y P25.

$$
W_{i} \leq \gamma \cdot P_{i} \quad \forall i \in I
$$

La Figura 5.10. reúne los diseños de red intermodal obtenidos al modelar las pérdidas. La nueva restricción asegura la incorporación de todos los nodos de origen en la solución, sin embargo, los diseños revelan distintos efectos según el nivel de capacidad. Los diseños con Cap1 no revelan ningún tipo de efecto al cambiar el valor del parámetro, por su parte, los resultados con Cap2 sí presentan diferencias. Con $\gamma=\{0.35\}$ se propicia la selección de un proyecto de primera milla que no es factible en ninguno otro diseño y se reduce el uso de la red por carretera, mientras que, con $\gamma=\{0.50\}$ los resultados muestran distintas alternativas seleccionadas y uso de las redes de transporte. Además, la comparación de los resultados de Cap2 con la Figura 5.4. muestra disparidades en los diseños. Entre estas, se reducen las características de las alternativas de capacidad instalada en algunas terminales para incrementar el número total de terminales localizadas y mejorar la alternativa vial de un proyecto de primera milla. Con la nueva terminal se cuantifican cambios importantes en los flujos finales debido a

\footnotetext{
${ }^{37}$ Estos valores se definen a partir del parecer de los agricultores y grupos de interés por participar en el mercado.
} 
la incorporación de la red ferroviaria y la reducción de los flujos fluviales. En cualquier caso, es posible que los efectos se acentúen más en las decisiones tácticas. 
Figura 5.10. Decisiones estratégicas en las soluciones de DERTI con restricción de pérdidas de producto

Soluciones DERTI con Dem1 y P25

PInv

$\mathrm{P}$

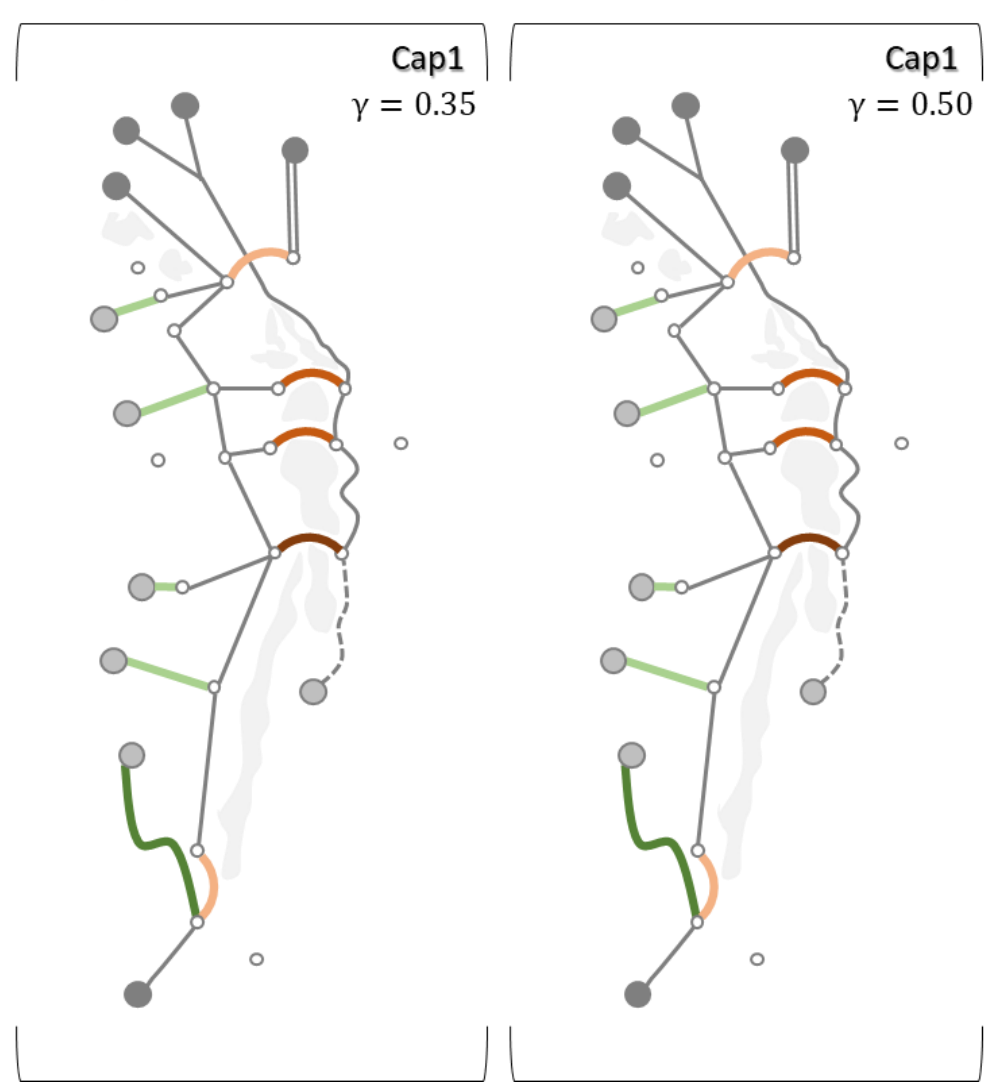

Soluciones DERTI con Dem1 y P25

Plnv
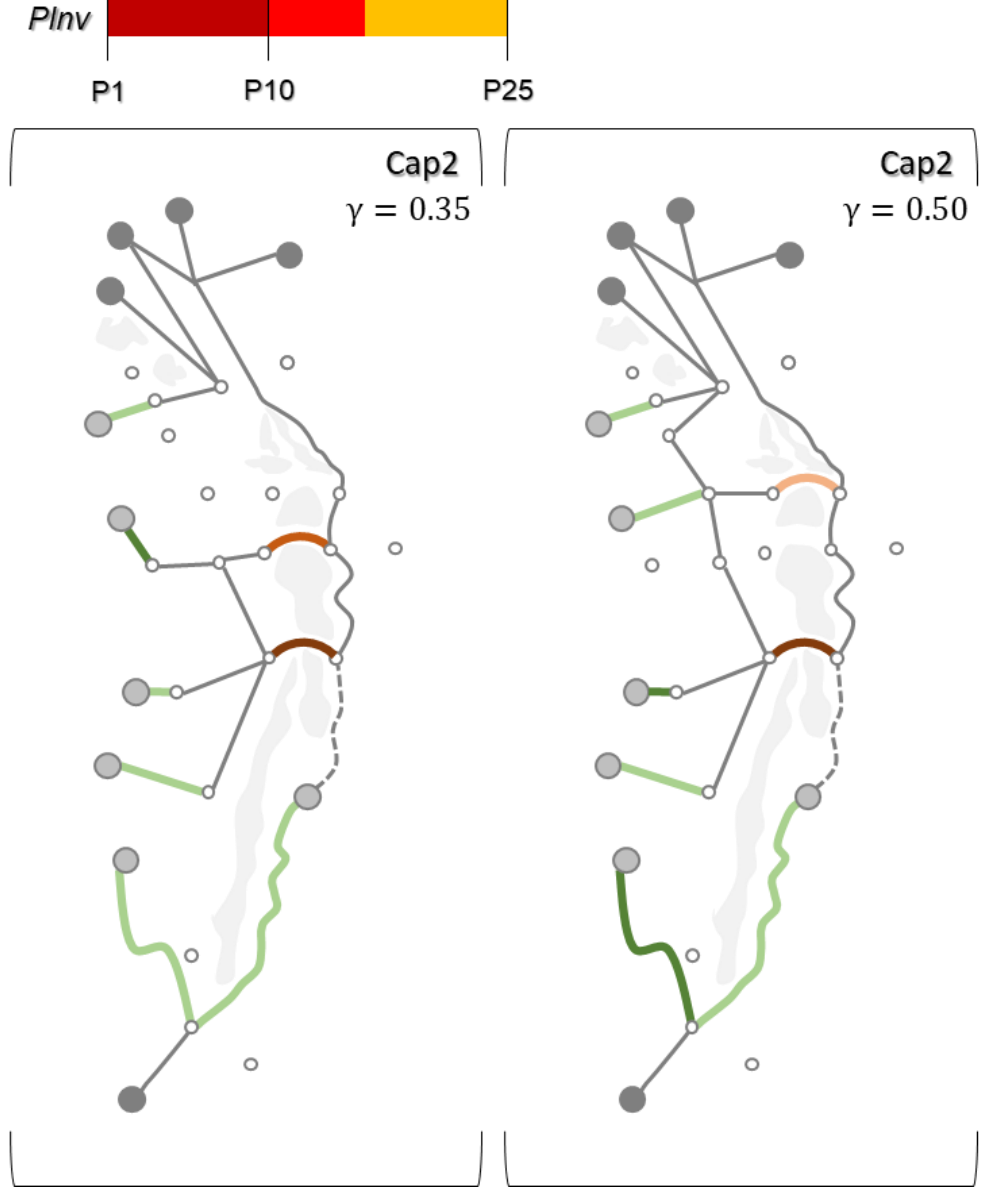

\begin{tabular}{|c|l|}
\hline \multicolumn{2}{|l|}{ Nodos } \\
\hline$\bigcirc$ & Origen \\
\hline & Destino \\
\hline \multicolumn{2}{|c|}{ Proyectos primera milla } \\
\hline- & Alter 1 \\
\hline- & Alter 2 \\
\hline- & Alter 3 \\
\hline Proyectos intermodales \\
\hline$\sim$ & Alter 1 \\
\hline$\frown$ & Alter 2 \\
\hline$\frown$ & Alter 3 \\
\hline Redes de transporte \\
\hline---- & Antiguos \\
\hline- & Carretera \\
\hline$\sim$ & Fluvial \\
\hline \hline & Ferroviario \\
\hline
\end{tabular}

Fuente: Elaboración propia 


\subsection{Discusión}

El MPLEM mono-periodo con enfoque económico desarrollado es una formulación genérica a un problema combinado LI-DR intermodal con limitaciones de financiación. El modelo permite localizar nuevos nodos y arcos en una red intermodal, al mismo tiempo que se decide la capacidad en los nuevos nodos, las características de los nuevos arcos y la asignación de los flujos que satisfacen la demanda. Aunque el modelo se inspiró en la situación de la ZODES Magdalena Medio, que se describió en el capítulo 3, se puede aplicar en otros contextos o regiones para diseñar las redes de aprovisionamiento de materias primas, e inclusive, se pueden incorporar otras redes subyacentes o reducirlas. De igual manera, el modelo es útil para decidir la localización y asignación de varios tipos de instalaciones que concentren la oferta de servicios de apoyo para el comercio y la población (escuelas, hospitales, etc.), donde cada red subyacente puede ser interpretada propiamente como una red de transporte o como una conexión no física interpretada en función de nodos y arcos.

La gran mayoría de los problemas en la literatura asumen intrínsecamente que la inversión total requerida está disponible, lo que no es tan cierto en situaciones del mundo real. La formulación atiende a esta realidad, analizando los efectos en las decisiones cuando varía la disponibilidad de inversión de manera conjunta con variaciones en la demanda y la capacidad de las alternativas intermodales. La limitación de financiación pone de manifiesto la complejidad del proceso al que se enfrentan los tomadores de decisión, en este caso de los actores públicos, para optimizar la asignación de los recursos económicos y obtener los mayores beneficios para todas las partes involucradas. Los resultados obtenidos determinan que el impacto es satisfactorio siempre que se establezcan factores clave del sistema que impliquen una asignación razonable del presupuesto. Por ejemplo, para la zona de estudio la consideración de las capacidades Cap2 no solo flexibilizan la decisión de inversión, igualmente, capturan con cierto grado de satisfacción los posibles cambios de la demanda que de alguna manera permite valorar el uso de las infraestructuras y ratificar la necesidad de invertir en tales proyectos.

La valoración de la capacidad en la red, desde una perspectiva endógena, marca pautas en el diseño intermodal y en el uso de la misma. Con una visión sesgada a la ZODES Magdalena Medio, el exceso de oferta puede tener efectos adversos que reducen la satisfacción en la asignación del presupuesto, ya que los impactos no se equilibran en toda la región. La exclusión de uno o más nodos de origen de la solución puede incrementar las desigualdades intrarregionales y dificultar el desarrollo de la subregión. El reducido uso de la capacidad instalada en algunas terminales desestima ciertos requerimientos de inversión en la red. El uso de las redes subyacentes se ve rezagado con disponibilidades de inversión que tienden a la menor disponibilidad. Estos resultados pueden repercutir en la gestión del presupuesto, pues no fortalecen la conectividad de la zona y tampoco valorizan las inversiones previas en infraestructura de transporte. Las pérdidas de producto, más que una limitación al diseño de la red, debe entenderse como una estrategia de crecimiento territorial para impulsar la economía, fortalecer el sector agrícola e impulsar la integración de la subregión en el mercado nacional.

En ese sentido parece interesante extender el MPLEM mono-periodo en distintas direcciones. Por ejemplo, introduciendo múltiples periodos de decisión para analizar la planificación de la red en el tiempo y satisfacer el problema de investigación abordando de manera conjunta las tres dimensiones de sostenibilidad. En la planificación a largo plazo se 
podría explorar los efectos económicos en el diseño gradual de la red intermodal y los cambios en la selección y programación de los proyectos de inversión a la luz del presupuesto disponible. De acuerdo con las particularidades identificadas en la ZODES Magdalena Medio y a la práctica habitual en estos tipos de proyecto de inversión, es interesante validar la asignación de los recursos económicos teniendo en cuenta las interdependencias entre los proyectos, los múltiples presupuestos de inversión y las compensaciones basada en la sostenibilidad de los proyectos. Con estas oportunidades para extender el trabajo no solo se llenan vacíos existentes en la literatura, de igual forma se ajusta la formulación aún más a la realidad del problema que se estudia en esta tesis. En los capítulos 6 y 7 se presentan los modelos de programación que atienden estas líneas de investigación.

Otras direcciones futuras del MPLEM mono-periodo pueden ser la introducción de un indicador que capture los desequilibrios entre los nodos de origen respecto a la asignación del presupuesto de inversión, la valoración de estrategias de cobertura de mercado vista desde las pérdidas de producto, y los flujos de múltiples productos. El inadecuado uso de la capacidad instalada también lleva a contemplar una restricción que paute el mínimo uso de capacidad para localizar una nueva terminal intermodal. Además, la estructura de la red de entrada se puede ampliar a una estructura jerárquica tanto en los nodos como en los arcos. La jerarquía en los nodos se puede definir con el propósito de conectar las terminales intermodales con otros nodos logísticos y analizar los efectos con las conexiones locales, regionales y nacional. La jerarquía en los arcos, tomando como referencia la ZODES, se puede entender conforme a las clasificaciones de las vías para los modos por carretera (primarias, secundarias y terciarias) y fluvial (corredores de carga y de pasajeros y mercancías) expuestas en el capítulo 2. Estas líneas de trabajo futuro tienen un enfoque sesgado a la planificación del territorio como una forma de propiciar acciones asertivas que estimulen el crecimiento de la región que se estudie.

\subsection{Conclusiones}

La capacidad y los costes son factores preponderantes para la eficiencia económica y la calidad del servicio en los sistemas intermodales. En el problema combinado LI-DR intermodal que se estudia en esta tesis se abordan estos factores con miras a superar ciertas lagunas en la literatura. El primero de ellos desde una perspectiva endógena vinculada a las decisiones de localización y el segundo en contraposición a los requerimientos de inversión para la toma de decisiones en el diseño de la red intermodal. Por ello, el MPLEM mono-periodo presentado en este capítulo incorpora, además de las habituales decisiones estratégico-tácticas de localización y asignación, las decisiones de capacidad en las terminales intermodales, las características de las vías de primera milla y la limitación del presupuesto de inversión.

El análisis en el comportamiento de la solución óptima permitió obtener resultados en tres sentidos: costes de uso de la red, en las decisiones estratégicas del DERTI y, en términos de eficiencia, en las decisiones tácticas del DERTI. Respecto al primero se concluye que, la planificación endógena de la capacidad y el presupuesto de inversión tienen un impacto significativo en los costes de uso de la red, más aún cuando el presupuesto tiende a la menor disponibilidad. Según el tipo de capacidad que se defina se pueden capturar mejor los efectos de los costes frente posibles cambios en la demanda, con capacidades amplias las diferencias no superan el $1.1 \%$. No obstante, los tomadores de decisiones deberán valorar las compensaciones para gestionar y garantizar una mayor disponibilidad de financiación e 
incrementar los beneficios entre los operadores de la red. En relación con las decisiones estratégicas del DERTI se deduce que, con presupuestos bajos se prioriza la localización de terminales intermodales, las características de la red mejoran cuando el presupuesto es mayor y el tipo de capacidad hace más o menos sensible la configuración modal de la red. Pese a ello y debido al exceso de oferta, la inversión no tiene un impacto directo en todos los nodos de origen.

Por su parte, tanto la capacidad instalada como las redes de transporte subyacentes tienen efectos en las decisiones tácticas del DERTI. El uso de la capacidad instalada en las terminales intermodales demuestra la injerencia del tipo de capacidad definida para las decisiones de localización y asignación. Con capacidades "ajustadas" se establece una configuración modal básica en el cual las decisiones de localización no se ven sustancialmente afectadas por el presupuesto de inversión, pero si se observa cómo se mejoran las alternativas a medida que se incrementa la disponibilidad. Con las capacidades "amplias" hay mayor flexibilidad que implica cambios en la selección y uso de las alternativas, inclusive, con disponibilidades de inversión próximas. No obstante, la flexibilidad puede convertirse en imprevisibilidad al profundizar el análisis para distintos escenarios de demanda, pues algunas terminales dejan de ser factibles para configurar la red y otras se hacen viables, pero cuando se dispone de montos de inversión específicos.

Por otro lado, los posibles desequilibrios que se pueden suscitar en la subregión a razón del excedente de producto que se pierde en los nodos de origen dan cuenta de la necesidad de validar la formulación y las soluciones de DERTI incorporando una nueva restricción al modelo. Aunque se comprobaron algunos efectos sobre este aspecto y se identificó de qué manera incide la pérdida de producto en las decisiones, es imprescindible extender la valoración incluyendo las pérdidas monetarias, así como otras pérdidas ocasionadas en el transporte y la consolidación del producto debido a su característica perecedera. Con este planteamiento se pretende integrar las actividades logísticas propias de la cadena de suministro con las decisiones de transporte y la planificación de la infraestructura básica en el territorio.

En general, los análisis presentados para el MPLEM mono-periodo proporcionan información vital para perfilar la toma de decisiones y direccionar la gestión de los tomadores de decisión. No obstante, presenta algunas limitaciones que merecen ser subsanadas a través de la recopilación y estandarización de datos que permitan acreditar y precisar los resultados de las decisiones estratégicas y abarcar otros aspectos del diseño táctico de la red como son la capacidad de los vehículos modales, el diseño de rutas y la estacionalidad de los productos agrícolas. 


$$
\text { CApítulo } 6
$$




\title{
Capítulo 6
}

\section{Una programación multi-periodo para el análisis de la interdependencia entre los proyectos de inversión y las múltiples fuentes de financiación en el diseño económico de la red intermodal}

\begin{abstract}
Resumen: en este capítulo se desarrolla y utiliza un modelo de programación lineal enteromixto (MPLEM) multi-periodo para analizar las soluciones de diseño económico de la red de transporte intermodal (DERTI) en un horizonte de planificación a largo plazo. La incorporación del horizonte de planificación en el diseño de la red intermodal conlleva a evaluar la transición en el tiempo de la solución de DERTI. El MPLEM multi-periodo se emplea como formulación base para validar dos condiciones del problema de decisiones: la interdependencia entre los proyectos de inversión y los múltiples presupuestos de inversión. En la primera se modela la interdependencia entre los proyectos con el propósito de relacionar los costes de construcción y garantizar el uso de las instalaciones. En la segunda, además de la interdependencia, se modela la participación de múltiples actores como financiadores de los proyectos con la finalidad de analizar los efectos en las decisiones. En general, los experimentos numéricos se hacen con el objetivo de explorar los efectos ante incrementos en los diferentes costes, presupuesto de inversión y variaciones en la demanda. Los análisis permiten intuir una jerarquía en las decisiones ligadas a la asignación de los recursos económicos y las posibilidades de no abordar o incluso abandonar decisiones de inversión, gestionando la cartera de proyectos y la participación de inversores privados.
\end{abstract}




\subsection{Introducción}

Un problema de decisiones que involucran la localización de instalaciones se resuelve como parte de un proceso de toma de decisiones del cual se espera que la solución conserve la vigencia durante un tiempo considerable (Alumur et al., 2016), pues, son decisiones difíciles de cambiar una vez implementadas y requieren de grandes inversiones que tienen efectos a largo plazo (Govindan et al., 2017). La irreversibilidad de la decisión junto a la vida económica de los proyectos (entre 20 años y un siglo) conducen necesariamente a evaluarlos en un amplio horizonte temporal (Wiegmans \& Behdani, 2018). Los resultados de la revisión sistemática de la literatura en dos etapas vinculadas (RSL-2EV) constatan que los problemas combinados de localización de instalaciones-diseño de red (LI-DR) escasamente se han formulado en un horizonte de planificación a largo plazo. De manera excepcional, Fernandes et al. (2013) lo hicieron para modelar la colaboración y uso compartido de las instalaciones.

En este capítulo se propone y utiliza un modelo de programación lineal entero-mixto (MPLEM) multi-periodo para analizar el comportamiento de la solución de diseño económico de la red de transporte intermodal (DERTI) que satisface el problema combinado LI-DR intermodal descrito en el capítulo 3. La formulación corresponde a la extensión multi-periodo del modelo presentado en el capítulo 5. Este se resuelve con el esquema de resolución Python+Gurobi (descrito en la subsección 3.5.2.) y se realizan experimentos numéricos para distintos escenarios generados con el objetivo de explorar los efectos en las decisiones. El análisis de las soluciones de DERTI multi-periodo pretenden entender cómo reacciona la solución a los cambios en los valores de los parámetros de costes, demanda y presupuesto de inversión. La extensión de la formulación se define para dos condiciones: la interdependencia entre los proyectos de inversión y los múltiples presupuestos de inversión. Con estas dos condiciones se contribuye al vacío identificado en la literatura respecto a las formulaciones de múltiples periodos de decisión en los problemas combinado LI-DR intermodal.

El capítulo está organizado de la siguiente manera. En la sección 6.2. se revisa la literatura relacionada con la interdependencia entre los proyectos y las múltiples fuentes de financiación. En la sección 6.3. se formula el MPLEM multi-periodo con enfoque económico que se emplea para validar dos condiciones del problema de investigación. En la sección 6.4. se analizan los resultados de los experimentos numéricos generados para distintos escenarios y se valida la formulación respecto a la primera condición: la interdependencia entre los proyectos de inversión para priorizar las decisiones de localización. Dado que los problemas combinados LI-DR son de interés para actores públicos y privados, en la sección 6.5. se validan los efectos de considerar múltiples fuentes de financiación en el MPLEM multi-periodo con interdependencias. En la sección 6.6. se desarrolla la discusión de los resultados y en la sección 6.7. se presentan las conclusiones de este capítulo.

\subsection{Revisión de la literatura}

En esta sección se analiza la literatura referida a las condiciones de interdependencia entre los proyectos de inversión y las múltiples fuentes de financiación, con el propósito de orientar el desarrollo y posterior validación del MPLEM multi-periodo. Para ello, la revisión de la literatura se extiende a todo el campo del transporte cuyas propuestas utilicen como técnica de análisis la programación matemática. La literatura se revisa con el propósito de identificar de 
qué manera se han modelado estas dos condiciones. En la subsección 6.2.1. se agrupa la literatura sobre la interdependencia entre los proyectos y en la subsección 6.2.2. la literatura relacionada con las múltiples fuentes de financiación.

\subsubsection{La interdependencia entre los proyectos de inversión}

La interdependencia entre los proyectos de inversión se aplica cuando los beneficios o costes de un proyecto dependen de qué otro u otros proyectos se implementen (Shayanfar et al., 2016). Generalmente, los proyectos que tienen ese grado de interdependencia se complementan o sustituyen entre sí y se ejecutan para un área geográfica o ámbito común (Hosseininasab et al., 2018). Por ello, se evalúan como portafolios de proyectos a partir de diversos criterios que permiten establecer una comparación entre los proyectos (Bagloee \& Tavana, 2012), y se seleccionan tantos proyectos como permitan los recursos financieros disponibles (Archer \& Ghasemzadeh, 1999).

La interdependencia entre los proyectos se ha modelado en la literatura con distintas finalidades. Algunas propuestas se han centrado en la funcionalidad del sistema de transporte y otras en la viabilidad de las inversiones. En el primer grupo se distingue la propuesta de Durango-Cohen \& Sarutipand (2007) y Bhattacharyya et al. (2011) quienes vinculan dos o más vías de una red para optimizar las decisiones de mantenimiento y reparación que contrarreste las interrupciones en el sistema y las pérdidas de rendimiento en las múltiples instalaciones de la red. Bagloee \& Tavana (2012) relacionan los proyectos de expansión de las carreteras existentes y construcción de nuevas carreteras para minimizar los tiempos de viaje en los flujos de pasajeros. Miralinaghi et al. (2020) conectan los proyectos con proximidad física para minimizar el retraso total del viaje en un horizonte de planificación a largo plazo. Kumar \& Mishra (2018) mejoran la capacidad de los arcos potenciales en una red, relacionando los costes para los usuarios durante la construcción de los proyectos con los beneficios para el usuario después de la hipotética construcción de estos.

En el segundo grupo se encuentra la propuesta de Peng et al. (2019), quienes aplican descuentos en los costes asumidos por los usuarios y proveedores del sistema para decidir la inversión en diferentes segmentos de las vías férreas de una red de transporte ferroviaria. Garay-Sianca \& Nurre-Pinkley (2021) interrelacionan el estado de uso de los arcos de la red para priorizar la inversión en proyectos de restauración de distintas redes de transporte. Ukkusuri \& Patil (2009) teniendo en cuenta la elasticidad de la demanda asocian la interdependencia entre los proyectos para evaluar la flexibilidad de retrasar, cambiar o abandonar las decisiones de inversión de los proyectos priorizados. Shayanfar et al. (2016) relacionan los proyectos para evaluar cuáles podrían ser económicamente injustificables al considerar la fase de implementación de los seleccionados. Shayanfar \& Schonfeld (2019) extienden la propuesta anterior para una red urbana bajo incertidumbres de demanda.

Por otra parte, la tendencia creciente en el despliegue de vehículos eléctricos conlleva a evaluar proyectos interdependientes entre los sistemas de transporte y el sistema de energía (Jawad \& Liu, 2020). Ding et al. (2020) relacionan estos dos sistemas para decidir la localización de las estaciones de carga. Kazemi et al. (2016) deciden la ubicación y el tamaño de los estacionamientos para maximizar el beneficio de las empresas de distribución eléctrica. Xiang et al. (2016) para el análisis de la expansión de la red y la ubicación de estaciones de 
carga formulan un modelo multi-objetivo que minimiza los costes económicos, y maximiza la utilización de las estaciones de carga y el nivel de confiabilidad. Wei et al. (2019) analizan distintos trabajos en los cuales la interdependencia se ha incorporado en las formulaciones con enfoque en el equilibrio de la red, la planificación de estaciones de carga y la planificación de la expansión de las carreteras.

Para modelar el MPLEM multi-periodo que se desarrolla en este capítulo respecto a la condición de interdependencia entre los proyectos de inversión, se aplica una reducción en los costes de construcción de algunas de las alternativas. La interdependencia se establece por proximidad geográfica entre los proyectos de terminales intermodales y de vías de primera milla, dando preferencia en la reducción de los costes a los segundos siempre que se cumplan con la precedencia en la localización de los primeros conforme a la relación establecida entre estos. En la subsección 6.4.2 se explica en detalle esta condición y se presentan los resultados del análisis de la solución.

\subsubsection{Múltiples fuentes de financiación: limitación del presupuesto de inversión}

En la subsección 5.2.2. se realizó una corta introducción acerca de la limitación del presupuesto de inversión en múltiples periodos. Esta limitación, en su representación más sencilla y frecuente, contempla que los costes de inversión de los proyectos priorizados en cada periodo están limitados a un presupuesto de inversión disponible para el respectivo periodo (Khodakarami et al., 2014). Desde esta perspectiva se han identificado dos formas en la literatura para modelar la limitación: la forma que distingue entre las fuentes de financiación y la que vincula las fases de construcción de los proyectos. Aunque se reconoce que ambas formas se han ido ajustando a ciertas cuestiones de la realidad, en esta subsección se profundiza solamente en la primera forma.

En el análisis de decisiones con múltiples fuentes de financiación se ha identificado que los recursos pueden provenir de fuentes públicas y privadas, o internas y externas (Penyalver et al., 2019). Peng et al. (2019) formulan la limitación de financiación considerando dos fuentes para calcular el presupuesto de inversión disponible por periodo, uno corresponde al presupuesto anual de las entidades gubernamentales y el otro a los fondos internos recaudados de las tarifas cobradas por la operación del sistema. Zhou et al. (2014) y Li et al. (2012) declaran un conjunto de programas de inversión a través de los cuales se pueden financiar los proyectos que se evalúan, es decir, cada proyecto puede ser priorizado por un único programa de inversión en un periodo especifico. Aunque la financiación proviene de distintas fuentes, no se distingue esta particularidad ya que las decisiones están indexadas a los programas de inversión definidos. En Nickel et al. (2012) la indexación se transforma para distinguir entre los potenciales inversores externos y los préstamos especiales a efectuar a través de diversas fuentes.

Para modelar las múltiples fuentes de financiación en el MPLEM multi-periodo con interdependencias entre los proyectos de inversión se incorporan nuevos parámetros y un conjunto de restricciones que permiten diferenciar en la solución el presupuesto de inversión público del privado. 


\subsection{Propuesta del MPLEM multi-periodo}

En esta sección se formula el MPLEM multi-periodo con enfoque económico que satisfacen el problema combinado LI-DR intermodal descrito en el capítulo 3. Dado que esta formulación es una extensión del modelo presentado en el capítulo 5, en la subsección 6.3.1. se precisa el ajuste realizado al modelo y se enuncian los supuestos básicos para esta variante. En la subsección 6.3.2. se indica la notación utilizada en la formulación matemática y en la subsección 6.3.3. se proporcionan las relaciones matemáticas que formulan el MPLEM multiperiodo.

\subsubsection{A juste al problema de investigación y los supuestos básicos}

Para formular el MPLEM multi-periodo es necesario ajustar el problema de decisiones descrito en el capítulo 3 al entorno de una toma de decisiones que implique múltiples periodos de decisión. Este ajuste es posible y coherente con la disponibilidad real de financiación de los actores gubernamentales para llevar a cabo las inversiones, pues, no es inmediata, sino que se proporciona año a año de acuerdo con la vigencia de los presupuestos anuales de los municipios que conforman la zona de estudio. Por lo tanto, con las decisiones estratégicas de localización se seleccionan (¿cuáles?) y programan (¿cuándo?) los proyectos de inversión a lo largo del horizonte de planificación, y con las decisiones tácticas de asignación se determinan los flujos para cada arco en cada periodo.

La aplicación de estos ajustes al problema de decisiones conlleva a adaptar algunos de los supuestos básicos definidos en el capítulo 5, por lo tanto, los supuestos para el MPLEM multiperiodo son los siguientes:

i. Los supuestos básicos (i) a (iv) y (vi) a (viii) definidos en la subsección 5.3.1. se adaptan a la toma de decisiones multi-periodo.

ii. En cada periodo de decisión se puede elegir una o varias alternativas del conjunto dado para localizar un proyecto, pero se debe cumplir el orden implícito en las alternativas tal y como se muestra en la Figura 6.1.

iii. Los costes de construcción se ajustan conforme a los valores definidos para $k \in K$, no obstante, la sumatoria de estos costes para las tres alternativas proporcionadas de un mismo proyecto equivale al coste de inversión de la alternativa Alter3 en el MPLEM mono-periodo.

iv. Cada alternativa de capacidad en los arcos intermodales y cada alternativa vial en los arcos de primera milla se construye una sola vez y en cualquier periodo de decisión. Éstas se utilizan desde el mismo periodo en el que se construyen y se mantiene su uso por el resto del horizonte de planificación.

v. Los proyectos que se planifican en cada periodo de decisión están limitados por el presupuesto de inversión para el respectivo periodo de decisión. 
Figura 6.1. Modelado multi-periodo de las alternativas de capacidad/vial de los proyectos de inversión

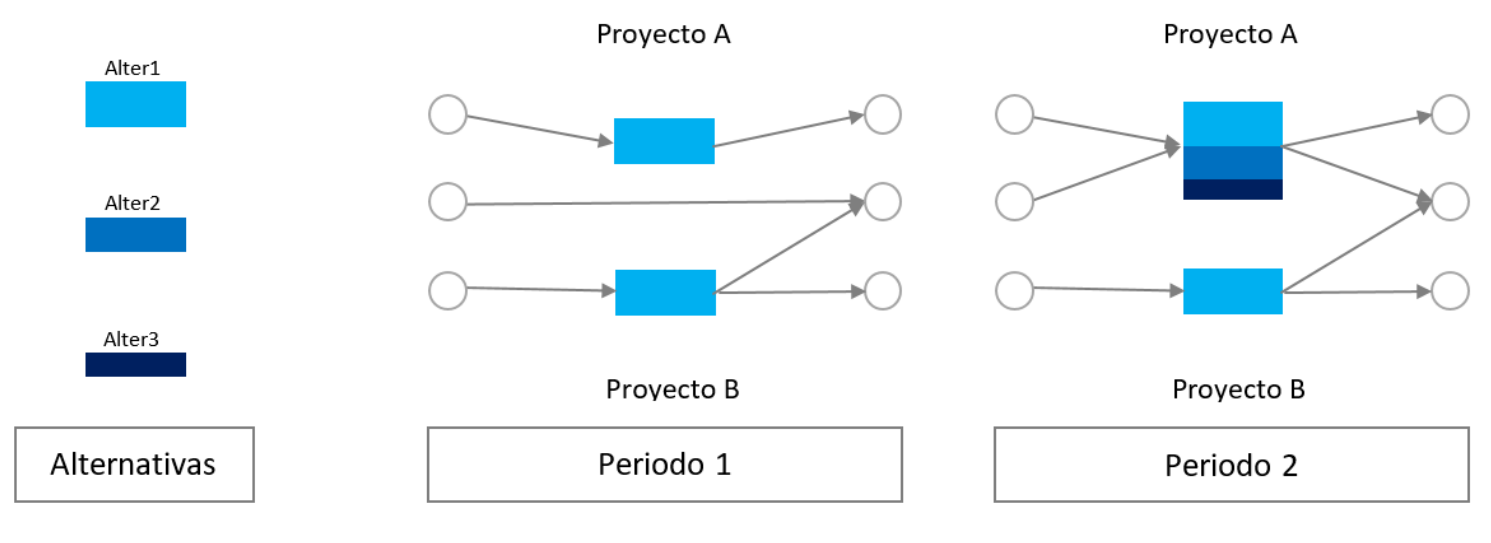

Fuente: Elaboración propia

\subsubsection{Notación del MPLEM multi-periodo}

Para el MPLEM multi-periodo se mantiene la estructura de la red de entrada como un grafo dirigido $G=(N, A)$. No obstante, los ajustes al problema de decisiones conducen a incorporar el conjunto $T$ para indexar los periodos en la formulación, lo que a su vez requiere la actualización de la nomenclatura de algunos parámetros y de las variables de decisión. La Tabla 6.1. muestra la notación actualizada. Los demás que no se relacionan en la tabla se conserva la descripción expuesta en la subsección 5.3.2.

Tabla 6.1. Notación complementaria para el MPLEM multi-periodo

\section{Conjuntos}

T Periodos de decisión

\section{Parámetros}

$P_{i}^{t} \quad$ Cantidad de producto disponible en el nodo de origen $i \in I$ en el periodo $t \in T$

$D_{j}^{t} \quad$ Cantidad de producto requerido en el nodo de destino $j \in J$ en el periodo $t \in T$

Ctte $_{k, a}^{t}$ Coste unitario de transporte para la alternativa $k \in K$ del arco $a \in A_{1}$ en el periodo $t \in T$

Ctte $e_{a}^{t}$ Coste unitario de transporte del arco $a \in A_{2} \cup A_{3} \cup A_{5}$ en el periodo $t \in T$

Coper $_{k, a}^{t}$ Coste unitario operativo para la alternativa $k \in K$ del arco $a \in A_{4}$ en el periodo $t \in T$

$P I n v^{t}$ Presupuesto de inversión disponible en el periodo $t \in T$

\section{Variables de Decisión}

$\beta_{k, a}^{t} \quad$ Toma el valor de 1 si se construye la alternativa $k \in K$ del $\operatorname{arco} a \in A_{1} \cup A_{4}$ en el periodo $t \in T$; 0 en caso contrario

$\delta_{k, a}^{t} \quad$ Toma el valor de 1 si se utiliza la alternativa $k \in K$ del $\operatorname{arco} a \in A_{1} \cup A_{4}$ en el periodo $t \in T ; 0$ en caso contrario

$\hat{X}_{k, a}^{t} \quad$ Cantidad de producto que fluye por la alternativa $k \in K$ del $\operatorname{arco} a \in A_{1} \cup A_{4}$ en el periodo $t \in T$ 


\subsubsection{Formulación del MPLEM multi-periodo}

Para construir el MPLEM multi-periodo con enfoque económico se incorpora el conjunto de periodos de decisión $T$ en el modelo presentado en el capítulo 5. Para esta formulación se omiten las pérdidas de producto, por lo tanto, la correspondiente variable de decisión se elimina del modelo. El MPLEM multi-periodo minimiza los costes de transporte y operativos de la red intermodal en el horizonte de planificación. El modelo permite seleccionar y programar los proyectos de inversión de primera milla y de terminales intermodales que reducen los costes de uso de la red, al mismo tiempo que deciden las capacidades con la que operan las terminales intermodales, las características de las vías de primera milla y los flujos en cada arco modal de la red para cada periodo. La formulación del modelo matemático es la siguiente:

$$
\min \sum_{t \in T} \sum_{k \in K} \sum_{a \in A_{1}} C t t e_{k, a}^{t} \cdot \hat{X}_{k, a}^{t}+\sum_{t \in T} \sum_{a \in A_{2} \cup A_{3} \cup A_{5}} C t t e_{a}^{t} \cdot \breve{X}_{a}^{t}+\sum_{t \in T} \sum_{k \in K} \sum_{a \in A_{4}} \operatorname{Coper}_{k, a}^{t} \cdot \hat{X}_{k, a}^{t}
$$

Sujeto a:

$$
\begin{aligned}
& \sum_{k \in K} \sum_{a \in A_{1}} \varphi_{a} \cdot \hat{X}_{k, a}^{t}+\sum_{a \in A_{2}} \varphi_{a} \cdot \breve{X}_{a}^{t}=P_{i} \quad \forall i \in I ; t \in T \\
& \sum_{a \in A_{5}} \alpha_{a} \cdot \breve{X}_{a}^{t}=D_{j} \quad \forall j \in J ; t \in T \\
& \varphi_{a} \cdot \breve{X}_{a \mid(e, s)}^{t}+\varphi_{a} \cdot \hat{X}_{k, a \mid(e, s)}^{t}=\alpha_{a} \cdot \breve{X}_{a \mid(e, s)}^{t}+\alpha_{a} \cdot \hat{X}_{k, a \mid(e, s)}^{t} \quad \forall a \in A ; k \in K ; t \\
& \in T \\
& \hat{X}_{k, a}^{t} \leq M \cdot \delta_{k, a}^{t} \quad \forall k \in K ; a \in A_{1} ; t \in T \\
& \hat{X}_{k, a}^{t} \leq \operatorname{Cap}_{k, a} \cdot \delta_{k, a}^{t} \quad \forall k \in K ; a \in A_{4} ; t \in T \\
& \delta_{k, a}^{t} \geq \delta_{k, a}^{t-1} \quad \forall k \in K ; a \in A_{1} \cup A_{4} ; t \in\{2, \ldots,|T|\} \\
& \delta_{k, a}^{t} \geq \delta_{k+1, a}^{t} \quad \forall k \in\{1, \ldots, K-1\} ; a \in A_{1} \cup A_{4} ; t \in T \\
& \beta_{k, a}^{t} \geq \delta_{k, a}^{t} \quad \forall k \in K ; a \in A_{1} \cup A_{4} ;\{t=1\} \in T \\
& \beta_{k, a}^{t} \geq \delta_{k, a}^{t}-\delta_{k, a}^{t-1} \quad \forall k \in K ; a \in A_{1} \cup A_{4} ; t \in\{2, \ldots,|T|\} \\
& \sum_{k \in K} \sum_{a \in A_{1} \cup A_{4}} \operatorname{Ccon}_{k, a} \cdot \beta_{k, a}^{t} \leq \operatorname{PInv}^{t} \quad \forall t \in T \\
& \beta_{k, a}^{t} \in\{0,1\} \\
& \forall k \in K ; a \in A_{1} \cup A_{4} ; t \in T \\
& \delta_{k, a}^{t} \in\{0,1\} \\
& \forall k \in K ; a \in A_{1} \cup A_{4} ; t \in T
\end{aligned}
$$




$$
\begin{array}{lr}
\hat{X}_{k, a}^{t} \geq 0 & \forall k \in K ; a \in A_{1} \cup A_{4} ; t \in T \\
\breve{X}_{a}^{t} \geq 0 & \forall k \in K ; a \in A_{2} \cup A_{3} \cup A_{5} ; t \in T
\end{array}
$$

La función objetivo (6.1) minimiza los costes de transporte y operativos a medida que se va diseñando la red intermodal en el horizonte de planificación. Las restricciones aplican para cada periodo de decisión. Las restricciones (6.2) establecen que el producto asignado desde un nodo de origen a través de las nuevas y antiguas vías de primera milla es equivalente a la cantidad de producto disponible en el respectivo nodo de origen. Las restricciones (6.3) determinan que el producto que se demanda en cada nodo de destino debe ser satisfecho en su totalidad. Las restricciones (6.4) establecen que el flujo en cada arco de la red debe estar balanceado. Las restricciones (6.5), (6.6) y (6.7) aseguran que el flujo asignado a los arcos de primera milla y a los arcos intermodales se efectúe, respectivamente, de acuerdo con la alternativa vial y la alternativa de capacidad que se decida utilizar en el horizonte de planificación. Las restricciones (6.8) establecen que las alternativas se pueden seleccionar para ser utilizadas siguiendo el orden predefinido. Las restricciones (6.9) precisan que para usar cada alternativa deben ser previamente construida. Las restricciones (6.10) aseguran que las alternativas se construyan una única vez en todo el horizonte de planificación. Las restricciones (6.11) garantizan que los costes de construcción de todas las alternativas seleccionadas y programadas para diseñar la red intermodal en cada periodo no excedan el presupuesto disponible del respectivo periodo. El conjunto de restricciones (6.12), (6.13), (6.14) y (6.15) establecen los dominios de las variables de decisión.

\subsection{Priorización de las decisiones de inversión ${ }^{38}$}

En esta sección se analiza el comportamiento de la solución de DERTI multi-periodo ante variaciones en los parámetros de costes, demanda y presupuesto de inversión, para lo cual se generan diferentes escenarios para explorar el problema. Los resultados se presentan en la subsección 6.4.1. De igual manera se extiende el análisis validando el modelo respecto a la condición de interdependencia entre los proyectos de inversión. En la subsección 6.4.2. se propone un nuevo conjunto de restricciones para incorporar la interdependencia en la formulación y se presentan los resultados de las validaciones realizados para esta variante. En ambos casos se utiliza el esquema de resolución expuesto en la subsección 3.5.2. Los valores aplicados para resolverlos se describen a continuación, no obstante, algunos se precisan en la respectiva subsección.

Para generar los diferentes escenarios se establecieron 5 periodos de decisión y se aplicaron los valores Dem1 y Cap2 ${ }^{39}$. El valor de Dem1 se vinculó al primer periodo de decisión y para los siguientes periodos se incrementó la demanda en $5 \%$ en cada uno. Las alternativas de capacidad $\operatorname{Cap}_{k, a}$ corresponden a 0.50 (Alter1), 0.33 (Alter2), 0.17 (Alter3). Por tanto, los costes de construcción de las alternativas se recalculan conforme al supuesto básico iii el cual hace referencia a que, la sumatoria de los costes de construcción de las tres alternativas de un

\footnotetext{
${ }^{38} \mathrm{El}$ análisis no profundiza en el comportamiento en los costes de uso de la red debido a que los efectos guardan similitud con lo expuesto en la subsección 5.4.1., es decir, a medida que se incrementa la disponibilidad de financiación se reducen los costes y con Cap2 se capturan mejor los cambios en la demanda.

${ }^{39}$ Estos valores se presentan en la Tabla 5.2., en la subsección 5.4.
} 
mismo proyecto equivale al coste de construcción de la alternativa Alter3 en el MPLEM monoperiodo. También se aplicaron distintos valores de costes y presupuestos de inversión. Para el primer periodo de decisión los valores aplicados a los costes corresponden a los valores que se emplearon en el MPLEM mono-periodo, y para los periodos siguientes se incrementó en 5\% en cada uno. Para visualizar el efecto de las limitaciones en la financiación se establecieron 20 presupuestos, sin embargo, para informar los resultados de esta sección se eligieron aquellos que permitieran explorar a profundidad los efectos en las decisiones. Los escenarios elegidos se precisan en cada subsección.

Los proyectos de inversión se identifican de la siguiente manera. Los proyectos de terminales intermodales TI1 a TI4 están configuradas por los modos carretera-fluvial, TI5 por los modos carretera-ferroviario y TI6 y TI7 por los modos fluvial-ferroviario. Los proyectos de primera milla $\mathrm{C} 1$ a $\mathrm{C} 8$ son vías de carretera y los proyectos $\mathrm{H} 1$ y $\mathrm{H} 2$ son fluviales.

\subsubsection{Sensibilidad en el DERTI multi-periodo}

Para analizar la solución en el DERTI multi-periodo se aplican los valores de presupuesto de inversión descritos en la Tabla 6.2 y los valores de los costes que se representan en la Tabla 6.3. Los porcentajes de presupuesto equivalen a la suma del coste de construcción de todos los proyectos potenciales ${ }^{40}$. Los costes pueden, o no, variar a lo largo del horizonte de planificación. Los escenarios generados se resolvieron realizando experimentos numéricos en los cuales se combinan estos valores. En la Tabla 6.4. se consolidan los valores aplicados a los parámetros.

Tabla 6.2. Porcentajes del presupuesto de inversión para el MPLEM multi-periodo

\begin{tabular}{lccccc}
\hline \multirow{2}{*}{ PInv $^{t}$} & \multicolumn{5}{c}{ Periodos } \\
\cline { 2 - 6 } & Periodo 1 & Periodo 2 & Periodo 3 & Periodo 4 & Periodo 5 \\
\hline Pres. 1 & $10 \%$ & $10 \%$ & $10 \%$ & $10 \%$ & $10 \%$ \\
\hline Pres. 2 & $9 \%$ & $7 \%$ & $9 \%$ & $4 \%$ & $4 \%$ \\
\hline
\end{tabular}

Tabla 6.3. Valores de los costes para el MPLEM multi-periodo

\begin{tabular}{lcccc}
\hline \multirow{2}{*}{ Tipo de coste } & \multicolumn{4}{c}{ Costes } \\
\cline { 2 - 5 } & Cos. 1 & Cos. 2 & Cos. 3 & Cos. 4 \\
\hline Construcción alternativas & $\mathrm{a}$ & $\mathrm{b}$ & $\mathrm{a}$ & $\mathrm{a}$ \\
\hline Transporte fluvial & $\mathrm{a}$ & $\mathrm{a}$ & $\mathrm{b}$ & $\mathrm{b}$ \\
\hline Transporte por carretera & $\mathrm{a}$ & $\mathrm{a}$ & $\mathrm{a}$ & $\mathrm{a}$ \\
\hline Demás costes & $\mathrm{a}$ & $\mathrm{a}$ & $\mathrm{a}$ & \\
\hline a: iguales en cada periodo; b: incremento 5\% a partir del segundo periodo & &
\end{tabular}

a: iguales en cada periodo; b: incremento 5\% a partir del segundo periodo

\footnotetext{
${ }^{40}$ En cada período el presupuesto se agota localizando las alternativas intermodales y las alternativas de primera milla.
} 
Tabla 6.4. Valores aplicados a los escenarios generados para el MPLEM multi-periodo

\begin{tabular}{lcccccccc}
\hline \multirow{2}{*}{$\begin{array}{l}\text { Valor } \\
\text { Parámetro }\end{array}$} & Exp. 1 & Exp. 2 & Exp. 3 & Exp. 4 & Exp. 5 & Exp. 6 & Exp. 7 & Exp. 8 \\
\cline { 2 - 8 } & Pres. 1 & Pres. 1 & Pres. 1 & Pres. 1 & Pres. 2 & Pres. 2 & Pres. 2 & Pres. 2 \\
\hline Presupuesto & Cos. 1 & Cos. 2 & Cos. 3 & Cos. 4 & Cos. 1 & Cos. 2 & Cos. 3 & Cos. 4 \\
\hline Costes & & &
\end{tabular}

La Figura 6.2. muestra las soluciones estratégicas de DERTI multi-periodo obtenidas al resolver el modelo para los ocho escenarios. Estos permiten determinar que, además del efecto implícito del número de alternativas que se pueden construir de cada tipo de proyecto, los principales efectos según la disponibilidad de presupuesto se producen en la priorización de los proyectos intermodales. En general prima la selección de instalaciones carretera-fluvial para configurar la red intermodal. Como se considera al menos un proyecto de primera milla para cada nodo de origen, los cambios no son tan notables para este tipo de proyectos. Los efectos se enmarcan mayoritariamente en el número de alternativas que se seleccionan y programan en un mismo arco. De acuerdo con las condiciones descritas para los ocho escenarios, los proyectos C2, C4, C8, TI4 y TI6 no se consideran oportunos para diseñar la red intermodal.

Como se percibe en los diseños de Exp. 1 y Exp. 5, en los cuales se asume igual valor en los costes en el tiempo, no se presentan cambios significativos en la estructura de la red intermodal al comparar los resultados para los dos grupos de presupuestos descritos en la Tabla 6.2. Aunque sí son apreciables algunas discrepancias en relación con el número de alternativas seleccionadas y el periodo en el que se programan, la mayoría de las decisiones son equiparables. La disponibilidad de financiación en los dos últimos periodos de Pres. 2 ocasiona en Exp. 5 que ciertas decisiones de construcción se anticipen como en C6, se posterguen como en H1, o se reduzca su prevalencia como en TI5 y TI7.

Por otro lado, al suponer un incremento por periodo de 5\% solamente en los costes de construcción de las alternativas, sí se producen cambios importantes en las decisiones. Los diseños de Exp. 2 y Exp. 6 no solo se distinguen entre sí, también difieren de Exp. 1 y Exp. 5, respectivamente. Confrontando los diseños de Exp. 2 y Exp. 6 se identifica la influencia que ejerce el presupuesto para validar la selección de un proyecto de inversión, como en TI1. Mientras que, el diseño de Exp 2. respecto a Exp 1. destaca que los proyectos C5 y TI2 son más apropiados para capturar el incremento en los costes de construcción.

Los cuatro diseños analizados anteriormente ponen de manifiesto la importancia de los proyectos que implican la participación del modo de transporte fluvial, en especial por las terminales intermodales, por tanto, en los siguientes se analizan los efectos en el incremento del 5\% en el coste transporte para este modo. Los diseños de Exp. 3 y Exp. 7 muestran efectos interesantes. Si bien tales efectos pueden ser más apreciables en las decisiones tácticas de asignación (como se muestra en la Figura 6.3.), es apreciable la importancia que adquiere el modo ferroviario en la configuración intermodal, pues se localizan las terminales intermodales TI5 y TI7. Pese al incremento del coste, la preferencia de estas dos terminales afianza la importancia de los proyectos de primera milla $\mathrm{H} 1$ y H2. En contraste, se analizan los efectos de incrementar solamente los costes de transporte por carretera en 5\%. Como se ilustra en los 
diseños de Exp. 4 y Exp. 8., este incremento no conlleva a cambios tan notables ya que los proyectos que diseñan la red intermodal se mantienen, algunas distinciones se aprecian en el número de alternativas que se priorizan, como por ejemplo H2. De igual manera, los diseños no distan sustancialmente de Exp. 1 y Exp. 5.

Por otra parte, la Figura 6.3. refleja las decisiones tácticas en las soluciones DERTI multiperiodo para los escenarios Exp. 1, Exp. 3, Exp. 5 y Exp. 7. Estas decisiones representan el flujo asignado en cada arco de la red de entrada. El flujo en los arcos de las redes de transporte se entiende como el porcentaje de la demanda que se asigna a través del arco, mientras que, para las terminales intermodales representa el porcentaje de uso de la capacidad instalada. Con menos disponibilidad de inversión se intensifica el flujo por carretera entre los nodos de origen y las terminales intermodales, y se diversifican los flujos en los arcos finales. Este efecto es notable al comparar Exp. 5 con Exp. 1. El incremento en el coste del transporte fluvial resulta en un diseño táctico diferenciado especialmente para Exp. 7, debido a que habilita arcos que no son factibles en otros diseños como por ejemplo C23 y C27, y sustituye la asignación de H5 hacía los arcos ferroviarios F1 y F2. El incremento del coste en este modo de transporte supone la localización de menos terminales intermodales, y a su vez de mayor uso de la capacidad instalada. 
Figura 6.2. Decisiones estratégicas en las soluciones de DERTI multi-periodo

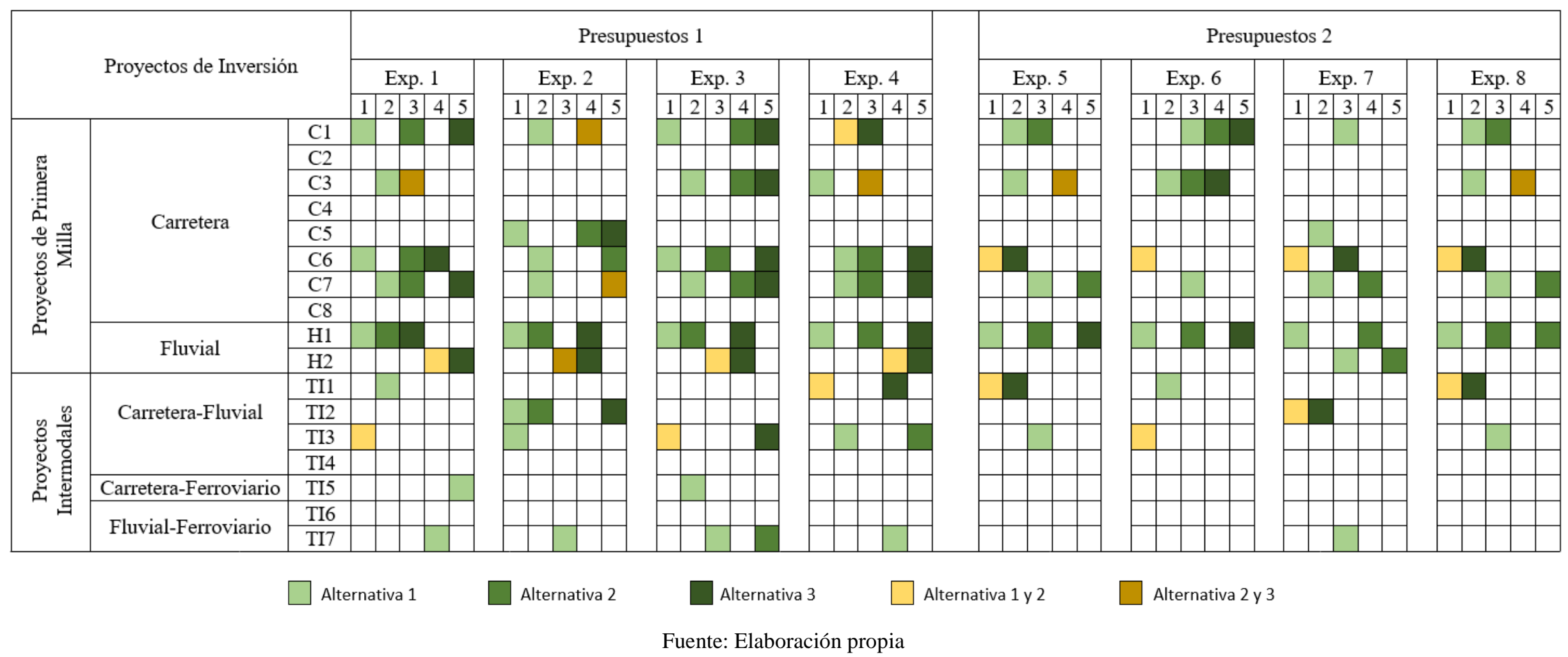


Figura 6.3. Decisiones tácticas en las soluciones de DERTI multi-periodo

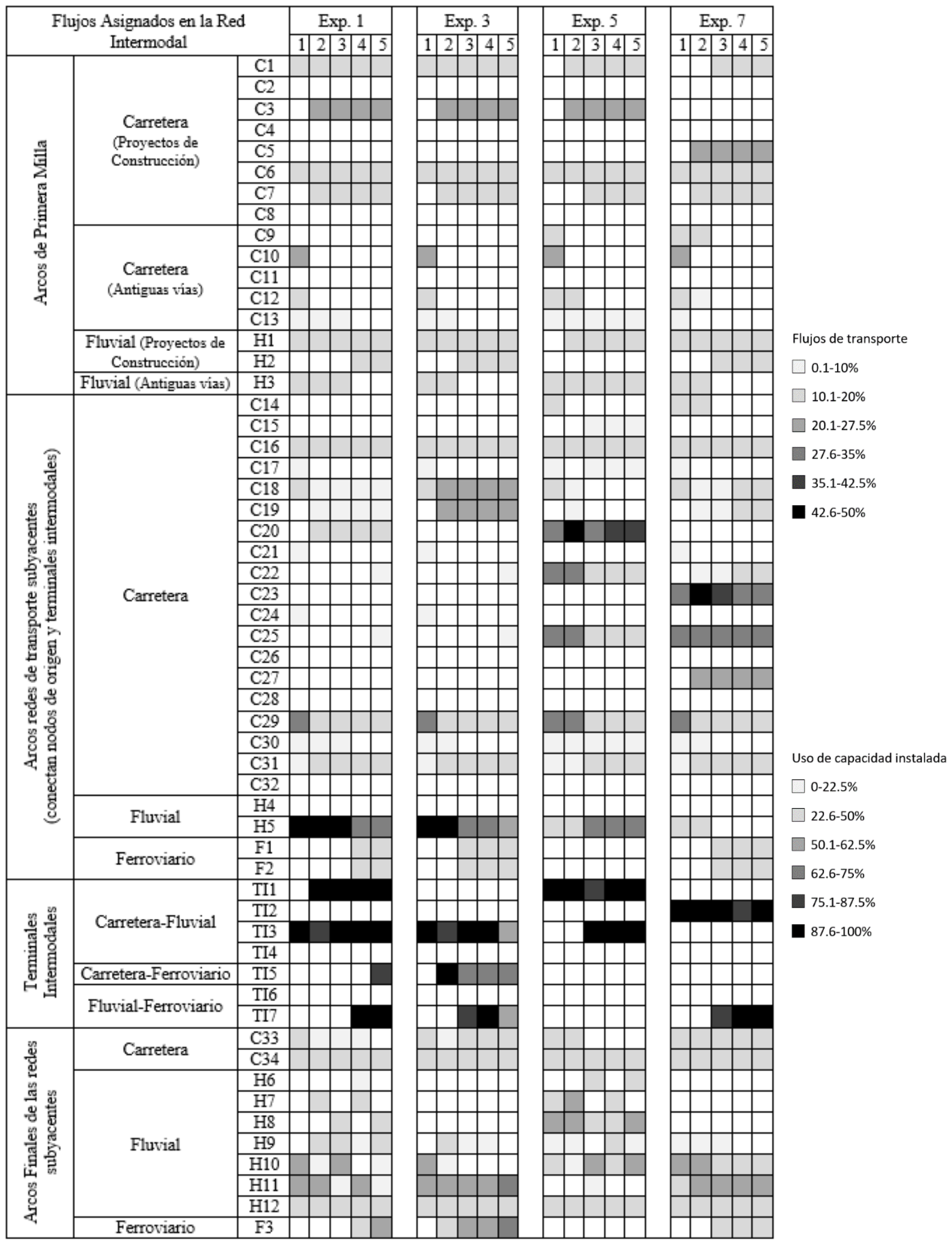

Fuente: Elaboración propia 


\subsubsection{Interdependencia en el diseño multi-periodo de la red intermodal}

En el mundo real los beneficios obtenidos y los costes incurridos en la construcción de cada proyecto de transporte suelen depender de la implementación de algunos otros proyectos (Shayanfar \& Schonfeld, 2019). Con la finalidad de modelar esta relación se utiliza el concepto de interdependencia entre los dos tipos de proyectos de inversión que se analizan para el problema combinado LI-DR intermodal: infraestructuras de transporte de primera milla y terminales intermodales. La interdependencia que se analiza en esta tesis refiere a una relación de precedencia por proximidad geográfica para seleccionar y programar primero los proyectos de terminales intermodales y después los proyectos de infraestructura de transporte de primera milla, aplicándose un descuento a los costes de construcción en los segundos si se cumple la precedencia en la localización de los primeros.

El modelado de la interdependencia implica que, en la formulación del MPLEM multiperiodo presentado en la subsección 6.3.3., se introduzcan las restricciones (6.16) y (6.17) y se reemplacen las restricciones (6.11) por las restricciones (6.18). El MPLEM multi-periodo con interdependencia entre los proyectos de inversión queda definido por las relaciones matemáticas (6.1) a (6.10) y (6.12) a (6.18). Las restricciones que permiten validar los proyectos con interdependencia son las siguientes:

$$
\begin{array}{cr}
\delta_{k, a_{4}}^{t} \geq \operatorname{rel}_{a_{4}, a_{1}} \cdot \omega_{k, a_{1}}^{t} & \forall k \in K ; a \in A_{1} \cup A_{4} ; t \in T \\
\beta_{k, a}^{t} \geq \omega_{k, a}^{t} & \forall k \in K ; a \in A_{1} ; t \in T \\
\sum_{k \in K} \sum_{a \in A_{1} \cup A_{4}} \operatorname{Ccon}_{k, a} \cdot \beta_{k, a}^{t}-\sum_{k \in K} \sum_{a \in A_{1}} \operatorname{Desc}_{k, a} \cdot \omega_{k, a}^{t} \leq \operatorname{PInv}^{t} & \forall t \in T
\end{array}
$$

Las restricciones (6.16) establecen que si se está utilizando previamente alguna de las alternativas de un proyecto intermodal y ésta tiene una relación de dependencia $\mathrm{rel}_{a_{4}, a_{1}}$ con uno o más proyectos de primera milla, es posible aplicar el descuento $\omega_{k, a_{1}}^{t}$ para asumir menores costes de construcción de los proyectos de primera milla. Las restricciones (6.17) aseguran que el descuento se aplique si la alternativa vial ha sido programada para su construcción. Las restricciones (6.18), de acuerdo con el presupuesto de inversión disponible en el periodo, limitan el número de alternativas de los proyectos que se pueden seleccionar y programar para construcción y los descuentos que se pueden aplicar a las alternativas de primera milla.

Para validar la formulación del MPLEM multi-periodo con interdependencias entre los proyectos se tiene en cuenta las relaciones de precedencia de los proyectos de inversión que se muestran en la Tabla 6.5. Dado que cada proyecto se caracteriza por tres alternativas los descuentos aplicables a los proyectos de primera milla equivalen, respectivamente, a 5\%, 7\% y $10 \%$ de los costes de construcción. La Tabla 6.6. refleja cómo se emplean estos porcentajes. 
Tabla 6.5. Interdependencia entre los dos tipos de proyectos de inversión

\begin{tabular}{lccccccc}
\hline Tipo de proyecto & \multicolumn{7}{c}{ Interdependencia entre los proyectos de inversión } \\
\hline Arco intermodal (Precede) & TI1 & TI2 & TI3 & TI4 & TI5 & TI6 & TI7 \\
\hline $\begin{array}{l}\text { Arco primera milla } \\
\text { (Sucede) }\end{array}$ & C1, C2 & $\begin{array}{c}\text { C3, C4, } \\
\text { C5 }\end{array}$ & C6, C8 & C7 & $\begin{array}{c}\text { C1, C2, } \\
\text { C3 }\end{array}$ & H1 & H1, H2 \\
\hline
\end{tabular}

Tabla 6.6. Porcentaje de descuento aplicable por alternativa priorizada

\begin{tabular}{ccccc}
\hline \multirow{2}{*}{ Porcentaje de descuento } & \multicolumn{3}{c}{ Proyectos de primera milla } \\
\cline { 3 - 5 } & & Alter1 & Alter2 & Alter3 \\
\hline \multirow{2}{*}{ Alter1 } & $5 \%$ & --- & --- \\
\cline { 2 - 5 } & Alter2 & --- & $7 \%$ & --- \\
\hline
\end{tabular}

La formulación se resolvió para cuatro escenarios, Exp. 9 a Exp. 12. Para ello, se tomaron los valores de los parámetros utilizados en Exp. 1 y se aplicaron en el modelo con interdependencia para obtener el diseño de Exp. 9, de esta misma manera en las otras tres resoluciones. Para valorar los efectos de la interdependencia en las soluciones, en la Figura 6.4. se reproducen las soluciones de DERTI multi-periodo sin interdependencia y con interdependencia. En las soluciones con interdependencia se puede observar que los proyectos priorizados conducen a diseños de red similares y se podría interpretar que el modelo es poco sensible a los cambios propuestos, sin embargo, no es un resultado concluyente porque el número de proyectos de primera milla que se evalúan en el modelo es reducido y la conectividad de la red de entrada es limitada.

Por otra parte, la aplicación de los descuentos destaca el predominio de los proyectos de primera milla $\mathrm{C} 5$ y $\mathrm{H} 2$, dada la interdependencia en la priorización de los proyectos intermodales TI2 y TI7, respectivamente. La priorización de los demás proyectos no se condiciona con el cumplimiento de la interdependencia, no obstante, sí ocasionan modificaciones en la programación de las alternativas de algunos arcos de primera milla, como en $\mathrm{C} 1$ y H1. De manera excepcional, proyectos como C3 y TI5 solo son justificables si se incrementan los costes de transporte fluvial. 


\section{Figura 6.4. Proyectos priorizados sin y con interdependencia en las soluciones DERTI multi-periodo}

\begin{tabular}{|c|c|c|c|c|c|c|c|c|c|c|c|c|c|c|c|c|c|c|c|c|c|c|c|c|c|c|c|c|c|c|c|c|c|c|c|c|c|c|c|c|}
\hline \multirow{3}{*}{\multicolumn{3}{|c|}{$\begin{array}{c}\text { Proyectos de Inversión } \\
\text { Presupuesto } 10 \% \text { por periodo }\end{array}$}} & \multicolumn{18}{|c|}{ Sin Interdependencia } & \multicolumn{20}{|c|}{ Con Interdependencia } \\
\hline & & & \multicolumn{4}{|c|}{ Exp. 1} & \multicolumn{4}{|c|}{ Exp. 2} & \multicolumn{5}{|c|}{ Exp. 3} & \multicolumn{5}{|c|}{ Exp. 4} & \multicolumn{5}{|c|}{ Exp. 9} & \multicolumn{5}{|c|}{ Exp. 10} & \multicolumn{5}{|c|}{ Exp. 11} & \multicolumn{5}{|c|}{ Exp. 12} \\
\hline & & & 1 & 2 & $\begin{array}{ll}3 & 4 \\
\end{array}$ & 5 & 1 & $2 \mid 3$ & 4 & 5 & $1 \mid$ & 2 & 3. & 45 & & \begin{tabular}{|l|}
1 \\
\end{tabular} & 2 & $3 \mid 2$ & $\begin{array}{ll}4 & 5 \\
\end{array}$ & 5 & 1 & 2 & \begin{tabular}{|l|l}
3 \\
\end{tabular} & 4 & 5 & 1 & $2 \mid$ & \begin{tabular}{|l|l|l}
3 & 2 \\
\end{tabular} & $\begin{array}{ll}4 & 5 \\
\end{array}$ & & 1 & $2 \mid$ & \begin{tabular}{l|l}
3 & 2 \\
\end{tabular} & 455 & 5 & 1 & 2 & 3 & & 5 \\
\hline \multirow{10}{*}{ 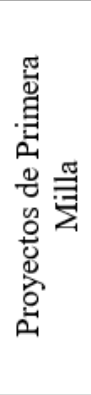 } & \multirow{8}{*}{ Carretera } & $\mathrm{C} 1$ & & & & & & & & & & & & & & & & & & & & & & & & & & & & & & & & & & & & & & \\
\hline & & $\mathrm{C} 2$ & & & & & & & & & & & & & & & & & & & & & & & & & & & & & & & & & & & & & & \\
\hline & & $\mathrm{C} 3$ & & & & & & & & & & & & & & & & & & & & & & & & & & & & & & & & & & & & & & \\
\hline & & $\mathrm{C} 4$ & & & & & & & & & & & & & & & & & & & & & & & & & & & & & & & & & & & & & & \\
\hline & & $\mathrm{C} 5$ & & & & & & & & & & & & & & & & & & & & & & & & & & & & & & & & & & & & & & \\
\hline & & $\mathrm{C} 6$ & & & & & & & & & & & & & & & & & & & & & & & & & & & & & & & & & & & & & & \\
\hline & & $\mathrm{C} 7$ & & & & & & & & & & & & & & & & & & & & & & & & & & & & & & & & & & & & & & \\
\hline & & $\mathrm{C} 8$ & & & & & & & & & & & & & & & & & & & & & & & & & & & & & & & & & & & & & & \\
\hline & \multirow{2}{*}{ Fluvial } & H1 & & & & & & & & & & & & & & & & & & & & & & & & & & & & & & & & & & & & & & \\
\hline & & $\mathrm{H} 2$ & & & & & & & & & & & & & & & & & & & & & & & & & & & & & & & & & & & & & & \\
\hline \multirow{7}{*}{ 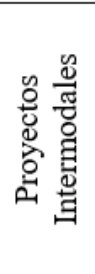 } & \multirow{4}{*}{ Carretera-Fluvial } & TI1 & & & & & & & & & & & & & & & & & & & & & & & & & & & & & & & & & & & & & & \\
\hline & & TI2 & & & & & & & & & & & & & & & & & & & & & & & & & & & & & & & & & & & & & & \\
\hline & & TI3 & & & & & & & & & & & & & & & & & & & & & & & & & & & & & & & & & & & & & & \\
\hline & & TI4 & & & & & & & & & & & & & & & & & & & & & & & & & & & & & & & & & & & & & & \\
\hline & Carretera-Ferroviario & TI5 & & & & & & & & & & & & & & & & & & & & & & & & & & & & & & & & & & & & & & \\
\hline & \multirow{2}{*}{ Fluvial-Ferroviario } & TI6 & & & & & & & & & & & & & & & & & & & & & & & & & & & & & & & & & & & & & & \\
\hline & & TI7 & & & & & & & & & & & & & & & & & & & & & & & & & & & & & & & & & & & & & & \\
\hline
\end{tabular}

Alternativas priorizadas

\begin{tabular}{|c|c|c|c|c|c|}
\hline Alternativa 1 & Alternativa 2 & Alternativa 3 & Alternativa 1 y 2 & Alternativa 2 y 3 & Alternativa 1,2 y 3 \\
\hline & rdependenci & & & & \\
\hline
\end{tabular}

Fuente: Elaboración propia 
Los anteriores resultados no pueden entenderse de manera generalizada ya que el DERTI multi-periodo con interdependencias está condicionado por el presupuesto de inversión y los costes, por tanto, los efectos de la interdependencia pueden variar según sea la disponibilidad de financiación y los costes de transporte y operativos que se apliquen. Desde esta perspectiva se analiza el comportamiento de la solución de DERTI multi-periodo con interdependencia para distintos presupuestos de inversión teniendo en cuenta los valores de costes aplicados en Exp. 9. La Tabla 6.7. muestra los porcentajes de inversión que se utilizan para ejecutar los distintos escenarios de inversión.

Tabla 6.7. Porcentajes del presupuesto de inversión para el MPLEM multi-periodo con interdependencias

\begin{tabular}{lcccccccc}
\hline \multirow{2}{*}{$\boldsymbol{P I n v}^{t}$} & \multicolumn{8}{c}{ Presupuestos de inversión } \\
\cline { 2 - 9 } & B1 & B2 & B3 & B4 & B5 & B6 & B7 & B8 \\
\hline Periodo 1 & $7.1 \% *$ & $8 \%$ & $9 \%$ & $10 \%$ & $11 \%$ & $7.1 \% *$ & $8 \%$ & $20 \%$ \\
\hline Periodo 2 & $7 \%$ & $8 \%$ & $9 \%$ & $10 \%$ & $11 \%$ & $10 \%$ & $3 \%$ & $18 \%$ \\
\hline Periodo 3 & $7 \%$ & $8 \%$ & $9 \%$ & $10 \%$ & $11 \%$ & $6 \%$ & $10 \%$ & $16 \%$ \\
\hline Periodo 4 & $7 \%$ & $8 \%$ & $9 \%$ & $10 \%$ & $11 \%$ & $4 \%$ & $3 \%$ & $14 \%$ \\
\hline Periodo 5 & $7 \%$ & $8 \%$ & $9 \%$ & $10 \%$ & $11 \%$ & $10 \%$ & $8 \%$ & $12 \%$ \\
\hline
\end{tabular}

${ }^{*}$ Mínimo requerimiento de inversión en el primer periodo para obtener una solución factible.

La Figura 6.5. muestra las soluciones de DERTI multi-periodo con interdependencia de acuerdo con los presupuestos de inversión B1 a B8, que corresponden a los escenarios Exp. 13 a Exp. 20. El diseño de Exp. 13 determina que con disponibilidades tan limitadas la interdependencia pierde relevancia en la priorización de los proyectos. Los recursos económicos se asignan sin considerar las relaciones, por tanto, no se aprovechan los descuentos a los proyectos de primera milla. Por su parte, en los diseños Exp. 14 a Exp. 17 la interdependencia adquiere importancia a medida que se incrementa la disponibilidad presupuestaria. En Exp. 14 el diseño marca la interdependencia entre TI3 con C6 y TI7 con H2. Con Exp. 15 la interdependencia se enmarca entre los proyectos TI2 con C5. Mientras, para Exp. 16 se suprimen algunas de las relaciones anteriores para anticipar las decisiones de TI7 con H2, y así ampliar la selección de las alternativas de C4. En el diseño Exp. 17, además de las terminales TI3 y TI7, se prioriza TI1 para obtener beneficios en la selección del proyecto C1.

En general se puede establecer que, la aplicación de la interdependencia está sujeta al presupuesto disponible en cada periodo, y el aprovechamiento de los descuentos conlleva a distintas configuraciones de red intermodal. Esta afirmación es más evidente en los diseños de Exp. 18 a Exp. 20 ya que la heterogeneidad de las disponibilidades en cada periodo marca profundas diferencias en las decisiones. Con Exp. 18 los descuentos se aprovechan en el mismo periodo que se programa el proyecto precedente, periodos en los cuales se dispone de la mayor proporción de inversión. Mientras, en Exp. 19 se anticipa la programación de los proyectos precedentes para aprovechar los descuentos en el siguiente periodo, el cual tiene menos 
disponibilidad. En Exp. 20 no solo se aprovechan los descuentos, sino que se decide gran parte del diseño de la red en los tres primeros periodos de decisión. Estos tres grupos de presupuesto resultan en tres diseños de red intermodal distintos. 
Figura 6.5. Proyectos priorizados en las soluciones DERTI multi-periodo con interdependencia para los presupuestos de inversión B1 a B8

\begin{tabular}{|c|c|c|c|c|c|c|c|c|c|c|c|c|c|c|c|c|c|c|c|c|c|c|c|c|c|c|c|c|c|c|c|c|c|c|}
\hline \multirow{2}{*}{\multicolumn{3}{|c|}{ Proyectos de Inversión }} & \multicolumn{4}{|c|}{ B1 } & \multicolumn{4}{|c|}{ B2 } & \multicolumn{4}{|c|}{ B3 } & \multicolumn{4}{|c|}{ B4 } & \multicolumn{4}{|c|}{ B5 } & \multicolumn{4}{|c|}{ B6 } & \multicolumn{4}{|c|}{ B7 } & \multicolumn{4}{|c|}{ B8 } \\
\hline & & & \multicolumn{4}{|c|}{ Exp. 13} & \multicolumn{4}{|c|}{ Exp. 14} & \multicolumn{4}{|c|}{ Exp. 15} & \multicolumn{4}{|c|}{ Exp. 16} & \multicolumn{4}{|c|}{ Exp. 17} & \multicolumn{4}{|c|}{ Exp. 18} & \multicolumn{4}{|c|}{ Exp. 19} & \multicolumn{4}{|c|}{ Exp. 20} \\
\hline & & & 1 & \begin{tabular}{l|l}
2 & 3 \\
\end{tabular} & \begin{tabular}{|l|l}
4 & \\
\end{tabular} & 5 & $1 \mid$ & \begin{tabular}{l|l|}
2 & 3 \\
\end{tabular} & 4 & 5 & 1 & \begin{tabular}{l|l}
2 & 3 \\
\end{tabular} & 4 & 5 & 1 & \begin{tabular}{|l|l|}
2 & 3 \\
\end{tabular} & 34 & 5 & 1 & \begin{tabular}{l|l}
2 & 3 \\
\end{tabular} & 4 & 5 & $1 \mid$ & \begin{tabular}{|l|l|}
2 & 3 \\
\end{tabular} & 4 & 5 & \begin{tabular}{|l|}
1 \\
\end{tabular} & \begin{tabular}{|l|l}
2 & \\
\end{tabular} & \begin{tabular}{l|l}
3 & 4 \\
\end{tabular} & \begin{tabular}{|l|l|}
4 & 5 \\
\end{tabular} & 1 & \begin{tabular}{l|l}
2 & 3 \\
\end{tabular} & 4 & 5 \\
\hline \multirow{10}{*}{ 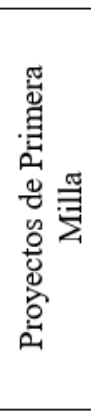 } & \multirow{8}{*}{ Carretera } & $\mathrm{C} 1$ & & & & & & & & & & & & & & & & & & & & & & & & & & & & & & & & \\
\hline & & $\mathrm{C} 2$ & & & & & & & & & & & & & & & & & & & & & & & & & & & & & & & & \\
\hline & & $\mathrm{C} 3$ & & & & & & & & & & & & & & & & & & & & & & & & & & & & & & & & \\
\hline & & $\mathrm{C} 4$ & & & & & & & & & & & & & & & & & & & & & & & & & & & & & & & & \\
\hline & & $\mathrm{C} 5$ & & & & & & & & & & & & & & & & & & & & & & & & & & & & & & & & \\
\hline & & $\mathrm{C} 6$ & & & & & & & & & & & & & & & & & & & & & & & & & & & & & & & & \\
\hline & & $\mathrm{C} 7$ & & & & & & & & & & & & & & & & & & & & & & & & & & & & & & & & \\
\hline & & $\mathrm{C} 8$ & & & & & & & & & & & & & & & & & & & & & & & & & & & & & & & & \\
\hline & \multirow{2}{*}{ Fluvial } & $\mathrm{H} 1$ & & & & & & & & & & & & & & & & & & & & & & & & & & & & & & & & \\
\hline & & $\mathrm{H} 2$ & & & & & & & & & & & & & & & & & & & & & & & & & & & & & & & & \\
\hline \multirow{7}{*}{ 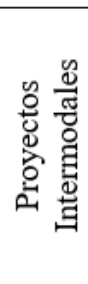 } & \multirow{4}{*}{ Carretera-Fluvial } & TI1 & & & & & & & & & & & & & & & & & & & & & & & & & & & & & & & & \\
\hline & & TI2 & & & & & & & & & & & & & & & & & & & & & & & & & & & & & & & & \\
\hline & & TI3 & & & & & & & & & & & & & & & & & & & & & & & & & & & & & & & & \\
\hline & & TI4 & & & & & & & & & & & & & & & & & & & & & & & & & & & & & & & & \\
\hline & Carretera-Ferroviario & TI5 & & & & & & & & & & & & & & & & & & & & & & & & & & & & & & & & \\
\hline & & TI6 & & & & & & & & & & & & & & & & & & & & & & & & & & & & & & & & \\
\hline & Fluvial-Ferroviario & TI7 & & & & & & & & & & & & & & & & & & & & & & & & & & & & & & & & \\
\hline
\end{tabular}

Alternativas priorizadas

\begin{tabular}{|c|c|c|c|c|c|}
\hline Alternativa 1 & Alternativa 2 & | Alternativa 3 & Alternativa 1 y 2 & Alternativa 2 y 3 & Alternativa 1,2 y 3 \\
\hline \multicolumn{6}{|c|}{ Iternativas priorizadas con interdependencia } \\
\hline
\end{tabular}




\subsection{Priorización con múltiples presupuestos de inversión de múltiples actores}

Dado que los problemas combinados LI-DR son de interés para los actores públicos y privados, la inversión para la puesta en marcha de los proyectos priorizados puede proceder del presupuesto de uno o más de estos actores (Ghaderi \& Jabalameli, 2013). Las disponibilidades de los actores públicos provienen naturalmente de los ingresos captados a través de impuestos y contribuciones especiales que son asumidos por diferentes figuras de contribuyentes, según las leyes de cada país (Penyalver et al., 2019). Es por ello que, la participación de actores privados en la financiación de los proyectos de infraestructura con la envergadura que analizamos en esta tesis requieren de la colaboración y coordinación con los actores públicos, para evitar que se eleve descontroladamente los costes aplicados a los usuarios y a la población a través de peajes o tarifas de usuarios (Trebilcock \& Rosenstock, 2015).

En esta sección se analiza el comportamiento de la solución de DERTI multi-periodo con interdependencias y múltiples presupuestos de inversión provenientes de diferentes fuentes de financiación: público y privado. La utilización del presupuesto privado acarrea distintos beneficios dependiendo el mecanismo de financiación que se adopte, no obstante, en este documento no es de interés ahondar en las compensaciones entre los mecanismos sino en reconocer un coste social vinculado a la utilización del presupuesto privado. El coste social que se contempla equivale a una proporción del coste de construcción de cada proyecto y se asume en el mismo periodo en el que se prioriza el proyecto.

Para llevar a cabo el análisis, primero se ajusta el MPLEM multi-periodo con interdependencias, introduciendo la condición de los múltiples presupuestos de inversión. En el modelo matemático el índice $L$ designa el conjunto de actores, el coste social se incorpora en la función objetivo y se ajustan los parámetros y variables de decisión que se ven afectados por el índice $l \in L$. La Tabla 6.8. muestra la nomenclatura actualizada. En los demás parámetros se mantiene la notación conforme a lo presentado en la subsección 6.3.2. Posteriormente, se describe la formulación del modelo que cobija la condición de múltiples presupuestos de inversión.

Tabla 6.8. Notación utilizada en el MPLEM multi-periodo con interdependencias y con múltiples presupuestos de inversión

\section{Conjuntos}

$L \quad$ Conjunto de actores $L=\{$ público,privado $\}$

\section{Parámetros}

PInv ${ }^{l, t}$ Presupuesto de inversión disponible del actor $l \in L$ en el periodo $t \in T$

$\theta_{k, a}^{l, t} \quad$ Coste social incurrido por construir la alternativa $k \in K$ en el arco $a \in A_{1} \cup A_{4}$ con el presupuesto de inversión del actor $\{l=$ privado $\} \in L$ en el periodo $t \in T$

\section{Variables de Decisión}

$\beta_{k, a}^{l, t} \quad$ Toma el valor de 1 si se construye la alternativa $k \in K$ del arco $a \in A_{1} \cup A_{4}$ con el presupuesto de inversión del actor $l \in L$ en el periodo $t \in T ; 0$ en caso contrario

$\omega_{k, a}^{l, t} \quad$ Toma el valor de 1 si se aplica el descuento a la alternativa $k \in K \operatorname{del} \operatorname{arco} a \in A_{1} \cup A_{4}$ que se construye con el presupuesto de inversión del actor $l \in L$ en el periodo $t \in T ; 0$ en caso contrario 
El MPLEM multi-periodo con interdependencia entre los proyectos y con múltiples presupuestos de inversión es el siguiente:

$$
\begin{aligned}
& \min \sum_{t \in T} \sum_{k \in K} \sum_{a \in A_{1}} \text { Ctte }_{k, a}^{t} \cdot \hat{X}_{k, a}^{t}+\sum_{t \in T} \sum_{a \in A_{2} \cup A_{3} \cup A_{5}} C t t e_{a}^{t} \cdot \breve{X}_{a}^{t} \\
& +\sum_{t \in T} \sum_{k \in K} \sum_{a \in A_{4}} \operatorname{Coper}_{k, a}^{t} \cdot \hat{X}_{k, a}^{t}+\sum_{l \in L} \sum_{t \in T} \sum_{k \in K} \sum_{a \in A_{1} \cup A_{4}} \theta_{k, a}^{l, t} \cdot \beta_{k, a}^{l, t}
\end{aligned}
$$

Sujeto a:

$$
\begin{aligned}
& (6.2)-(6.8),(6.13)-(6.15) \\
& \beta_{k, a}^{l, t} \geq \delta_{k, a}^{t} \quad \forall k \in K ; a \in A_{1} \cup A_{4} ; l \in L ;\{t=1\} \in T \\
& \beta_{k, a}^{l, t} \geq \delta_{k, a}^{t}-\delta_{k, a}^{t-1} \quad \forall k \in K ; a \in A_{1} \cup A_{4} ; t \in\{2, \ldots,|T|\} \\
& \sum_{l \in L} \sum_{t \in T} \beta_{k, a}^{l, t} \leq 1 \quad \forall k \in K ; a \in A_{1} \cup A_{4} \\
& \delta_{k, a_{4}}^{t} \geq \operatorname{rel}_{a_{4}, a_{1}} \cdot \omega_{k, a_{1}}^{l, t} \quad \forall k \in K ; a \in A_{1} \cup A_{4} ; l \in L ; t \in T \\
& \beta_{k, a}^{l, t} \geq \omega_{k, a}^{l, t} \quad \forall k \in K ; a \in A_{1} \cup A_{4} ; l \in L ; t \in T \\
& \sum_{k \in K} \sum_{a \in A_{1} \cup A_{4}} \operatorname{Con}_{k, a} \cdot \beta_{k, a}^{l, t}-\sum_{k \in K} \sum_{a \in A_{1}} \operatorname{Desc}_{k, a} \cdot \omega_{k, a}^{l, t} \leq \operatorname{PInv}^{l, t} \quad \forall l \in L ; t \in T \\
& \beta_{k, a}^{l, t} \in\{0,1\} \quad \forall k \in K ; a \in A_{1} \cup A_{4} ; l \in L ; t \in T \\
& \omega_{k, a}^{l, t} \in\{0,1\} \quad \forall k \in K ; a \in A_{1} \cup A_{4} ; l \in L ; t \in T
\end{aligned}
$$

La función objetivo (6.19) propone minimizar los costes de transporte y operativos en el horizonte de planificación, así como el coste social incurrido por priorizar los proyectos con el presupuesto de inversión de los actores privados. Las restricciones (6.2) a (6.8) y (6.13) a (6.15) conservan las respectivas descripciones presentadas en la subsección 6.3.3. Las restricciones (6.20), (6.21) y (6.22) establecen que, para utilizar una alternativa ésta debe ser previamente construida una única vez en el horizonte de tiempo y con el presupuesto de inversión de uno de los actores. Las restricciones (6.23) aseguran la interdependencia entre los proyectos según el actor del cual procedan los recursos financieros. Las restricciones (6.24) garantizan que el descuento se efectúe en el mismo periodo en el que la alternativa se programa para construcción. Las restricciones (6.25) determinan que el número de alternativas que se priorizan por cada actor está limitado por el presupuesto de inversión de cada actor en cada periodo y por los descuentos que se pueden aprovechar. El conjunto de restricciones (6.26) y (6.27) establecen los dominios de las variables de decisión. 
El MPLEM multi-periodo con interdependencias y con múltiples presupuestos de inversión se resuelve a partir de experimentos numéricos ejecutados para distintos escenarios de inversión, con el objetivo de explorar los efectos en la solución óptima ante cambios en el presupuesto de inversión de cada actor y cambios en el coste social. Estos análisis se presentan en la subsección 6.5.1. y la subsección 6.5.2., respectivamente. Los resultados obtenidos en cada instancia para la función objetivo se normalizan aplicando la función de transformación (6.28) utilizada por Marler \& Arora (2004). $F_{i}^{o}$ representa el valor más alto obtenido para la función objetivo y $F_{i}^{\max }$ el valor más bajo. Esto indica que las soluciones óptimas situadas en el valor 0 corresponden a la solución óptima con el coste de uso de la red más favorable, mientras que las situadas en el valor 1 corresponden a la solución óptima con el coste de uso de la red menos favorable.

$$
F_{i}^{\text {trans }}=\frac{F_{i}(X)-F_{i}^{o}}{F_{i}^{\text {max }}-F_{i}^{o}}
$$

\subsubsection{Efectos en la solución según el presupuesto de inversión de cada actor}

Para analizar los efectos en la solución ante las variaciones en la disponibilidad de financiación de cada actor se define un presupuesto global que es igual en cada periodo. El presupuesto global es un porcentaje de la sumatoria del coste de construcción de todos los proyectos de inversión. A partir de este presupuesto global se determinan las disponibilidades de cada actor. Los presupuestos de inversión de los actores varían en ${ }_{-}^{+} 0.1 \%$, conservando siempre la equivalencia para el respectivo presupuesto global. El modelo se resuelve para los presupuestos globales $9 \%, 10 \%, 11 \%$ y $12 \%$. Para establecer los descuentos aplicables por el cumplimiento de la interdependencia entre los proyectos, se detallan en la Tabla 6.9. los porcentajes para cada actor.

Tabla 6.9. Porcentaje de descuento aplicado a cada alternativa priorizada según cada actor

\begin{tabular}{|c|c|c|c|c|c|c|c|}
\hline \multirow{3}{*}{\multicolumn{2}{|c|}{$\begin{array}{c}\text { Porcentaje de } \\
\text { descuento }\end{array}$}} & \multicolumn{6}{|c|}{ Proyectos de primera milla } \\
\hline & & \multicolumn{3}{|c|}{ Actores públicos } & \multicolumn{3}{|c|}{ Actores privados } \\
\hline & & Alter1 & Alter2 & Alter3 & Alter1 & Alter2 & Alter3 \\
\hline \multirow{3}{*}{ 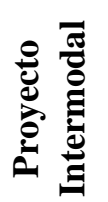 } & Alter1 & $3 \%$ & --- & --- & $5 \%$ & --- & --- \\
\hline & Alter2 & --- & $4 \%$ & --- & --- & $7 \%$ & --- \\
\hline & Alter3 & --- & --- & $5 \%$ & --- & --- & $10 \%$ \\
\hline
\end{tabular}

Para informar los resultados de esta subsección se ejecutaron 334 instancias que se representan en la Figura 6.6., de las cuales 319 son soluciones óptimas y las restantes son soluciones no factibles. En la Figura se grafican los valores normalizados del coste de uso de la red para los presupuestos globales mencionados anteriormente. En ambos extremos se indica el porcentaje de presupuesto de inversión de cada actor que permite obtener dicha solución. 
Los puntos indicados con recuadros amarillos se destacan con la finalidad de resaltar otras disponibilidades cuyas soluciones óptimas se sitúan cerca del valor más favorable. Las soluciones no factibles se pueden identificar en los espacios grises de la Figura 6.6.a. La no factibilidad en la solución y el comportamiento de las cuatro curvas resultan de la concurrencia de distintos aspectos del problema de decisiones. Por ejemplo, uno de ellos es que los costes de construcción de los proyectos de inversión no son iguales y la interdependencia se debe cumplir para cada actor, por lo tanto, al variar la disponibilidad de financiación de los actores cambia la factibilidad de priorizar cada alternativa que se evalúa.

La Figura 6.6.a. muestra que, con un presupuesto global de 9\% por periodo si el actor público participa con el $2.8 \%$ y el privado con el $6.2 \%$ se alcanzan costes de uso de la red poco favorables, mientras que los costes más favorables se obtienen con el $7 \%$ de participación pública y $2 \%$ del privado. Ante una situación restrictiva del presupuesto público, con la cual no se consigue garantizar el $7 \%$ en cada periodo, es preferible que este actor asuma compromisos de 5\%,6\% o 4\% ya que con estas participaciones los respectivos costes se sitúan cerca de la solución óptima más favorable. Las soluciones no factibles ponen de manifiesto la relevancia del presente análisis, y a su vez destacan que en ocasiones específicas más participación del actor público no conlleva a mayores reducciones en los costes. En el párrafo anterior se mencionó un ejemplo para esta situación de no factibilidad de la solución. Otra posibilidad radica en los porcentajes de descuentos que se aplican por cada actor para las alternativas de los proyectos de primera milla, pues, estos inciden en la asignación de los recursos económicos especialmente para el actor público cuyos descuentos son menores.

La Figura 6.6.b., 6.6.c. y 6.6.d., además de identificar las participaciones de presupuesto público y privado que conducen a establecer las soluciones más y menos favorables, permiten apreciar los efectos a medida que se aumenta el presupuesto global. El porcentaje público necesario para alcanzar los costes más favorables se elevan en tanto el presupuesto global aumenta. Este efecto es evidente porque, como se analizó en la sección anterior, con más disponibilidad de financiación se anticipa la priorización de algunos proyectos; además que con más participación privada se eleva el coste social y por ende el valor de la función objetivo. Es importante destacar que la inversión privada es conveniente para cubrir las necesidades de infraestructura en la subregión, pero debe ser gestionada de manera controlada. En las Figuras también se destacan distintas participaciones para los actores públicos y privados que ofrecen soluciones de diseño de red con costes de uso de la red cercanos a la solución óptima más favorable.

A partir de este análisis se presenta en el siguiente apartado un análisis de las soluciones estratégicas de DERTI multi-periodo con interdependencias y con múltiples presupuestos de inversión. 
Figura 6.6. Valores normalizados del coste de uso de la red para el MPLEM multi-periodo con interdependencias y con múltiples presupuestos de inversión

a.

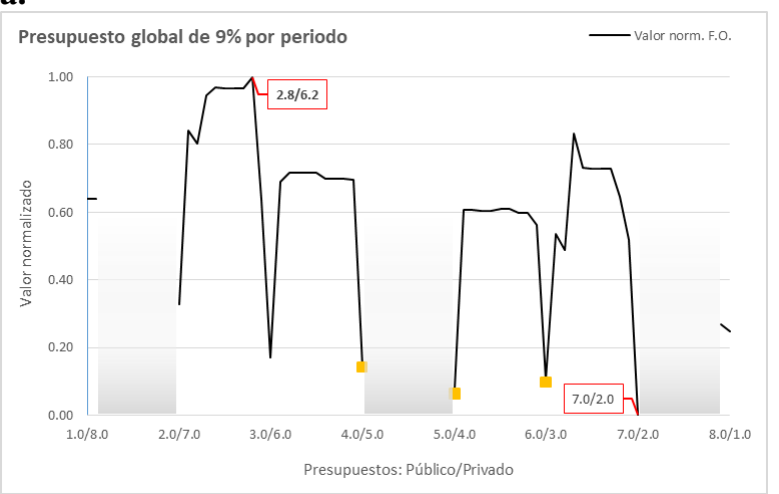

c.

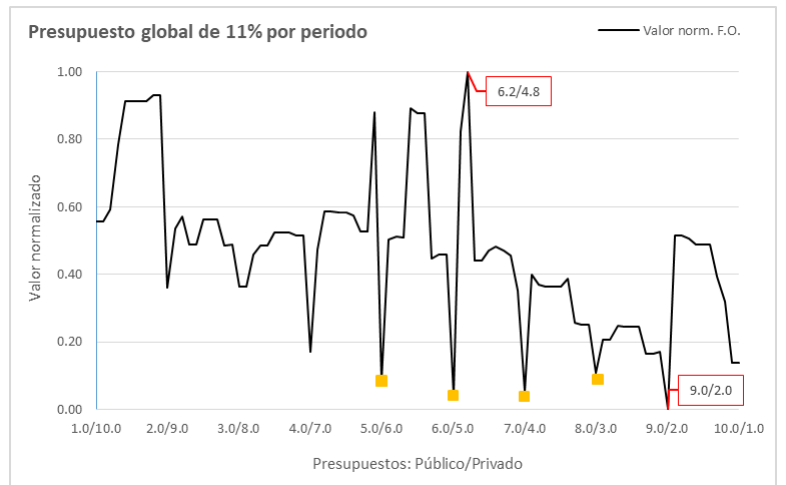

b.

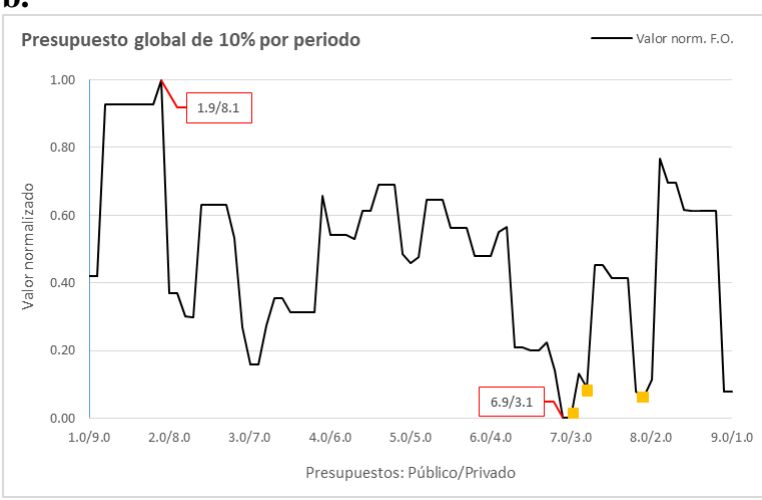

d.

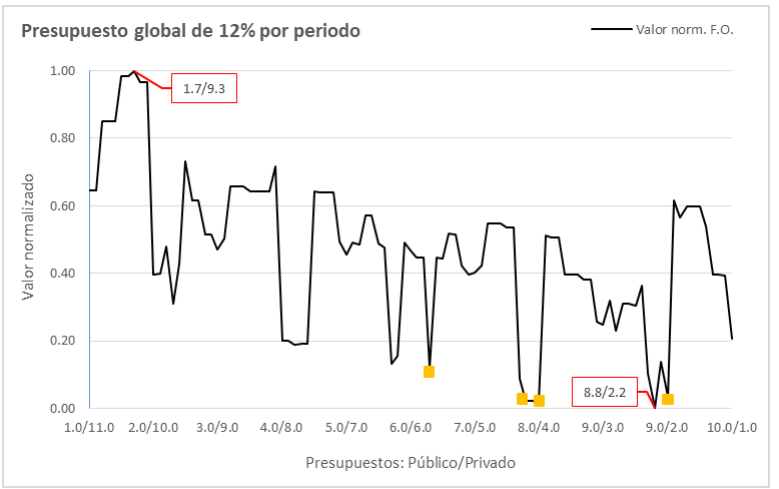

Fuente: Elaboración propia

\subsubsection{Actores y diseño de la red intermodal}

La Figura 6.7. representa las decisiones estratégicas para cuatro soluciones de DERTI multiperiodo con interdependencias y con múltiples presupuestos de inversión, donde se distinguen las alternativas que se priorizan de acuerdo con el presupuesto de inversión de cada actor. Los diseños revelan que la interdependencia entre los proyectos, en comparación con lo expuesto en la subsección 6.4.2., influye sustancialmente cuando está se cumple para cada uno de los actores que financian el diseño de la red. Sin embargo, con un presupuesto global del 9\% por periodo y con participaciones por actor según Exp. 21 y Exp. 22 se atenúa la priorización de las decisiones con interdependencia. Estas dos soluciones permiten contrastar que las participaciones por actor también inciden en la programación de las alternativas y en usar/desistir del presupuesto privado, especialmente en los dos últimos periodos de decisión. Una interpretación a este efecto puede entenderse desde el desbalance entre el coste social que se genera por localizar un proyecto adicional y los costes de transporte que se ocasionan con las condiciones actuales de los arcos de primera milla.

Los diseños de Exp. 22, Exp. 23 y Exp. 24 permiten confrontar cómo se priorizan las decisiones a medida que se incrementa únicamente la inversión pública. Los cambios en el presupuesto de inversión de este actor provocan cambios sutiles en el diseño de la red intermodal, como se percibe con los proyectos $\mathrm{C} 4, \mathrm{C} 5, \mathrm{H} 1$ y TI7. La mayoría de los proyectos se priorizan con inversión mixta, sin embargo, cuando la participación pública se va limitando 
es el actor privado quien asume el mayor número de decisiones iniciales para diseñar la red. La interdependencia y el aprovechamiento de los descuentos conlleva a diseños de red con un elevado número de terminales intermodales, el cual requiere a futuro un análisis profundo sobre el uso de la capacidad instalada. 
Figura 6.7. Decisiones estratégicas en las soluciones de DERTI con interdependencias y con múltiples presupuestos de inversión

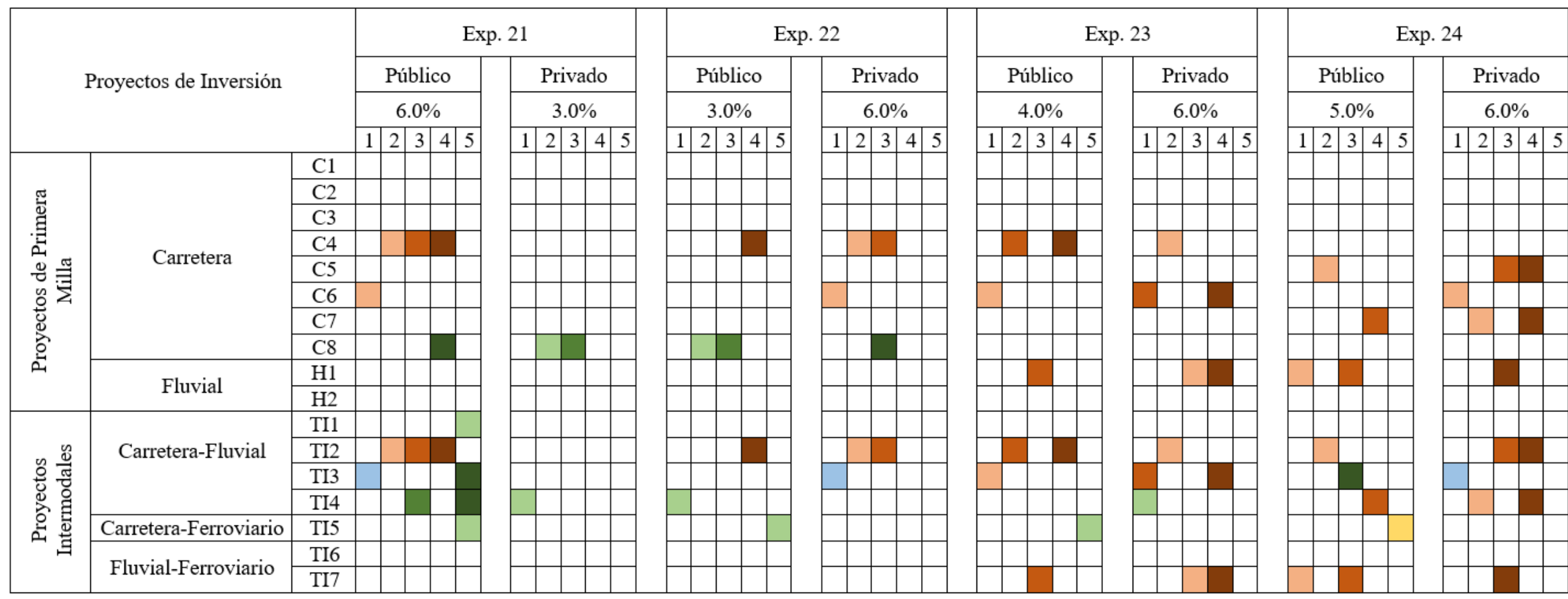

Alternativas priorizadas
$\square$ Alternativa 1
$\square$ Alternativa 2
Alternativa 3
$\square$ Alternativa 1 y 2
Alternativa 2 y 3
Alternativa 1, 2 y 3
Alternativas priorizadas con interdependencia
$\square$ Alternativa 1
$\square$ Alternativa 2
Alternativa 3
$\square$ Alternativa 1 y 2
Alternativa 2 y 3
Alternativa 1, 2 y 3

Fuente: Elaboración propia 


\subsubsection{Efectos en la solución según el coste social}

Los efectos en la solución óptima respecto a los cambios en los valores del coste social, en general, no son tan pronunciados. La Figura 6.8. muestra los valores normalizados de los costes de uso de la red para los cuatro presupuestos globales que se analizaron en la subsección anterior: $9 \%, 10 \%, 11 \%$ y $12 \%$. En cada figura se grafican los resultados para tres valores de coste social. Los efectos en la solución óptima son más notables a medida que el actor público reduce su porcentaje de inversión. Estos efectos se acentúan cuando el presupuesto global es mayor. Sin embargo, en todas las figuras se refleja cierto grado de semejanza entre las curvas. Dada esta semejanza es posible que los proyectos priorizados para diseñar la red intermodal sean los mismos en cada solución. La posibilidad de esta ocurrencia está sustentada en las características de la estructura de la red de entrada utilizada para validar el modelo, y por el número de proyectos de primera milla considerados. En otras palabras, con las características del grafo dirigido $G=(N, A)$ la solución óptima es poco sensible a los cambios en el coste social, por tanto, parece interesante validar el modelo en una red cuya estructura tenga otras características de conectividad.

Figura 6.8. Valores normalizados de la solución óptima para distintos valores de costes social

a.

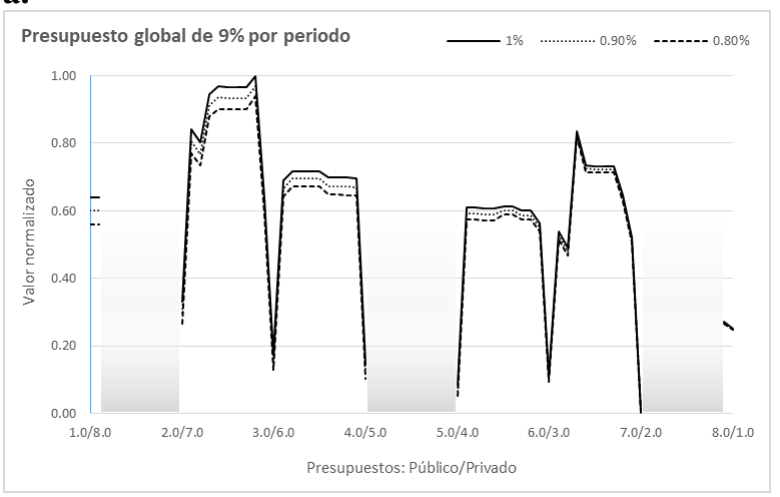

c.

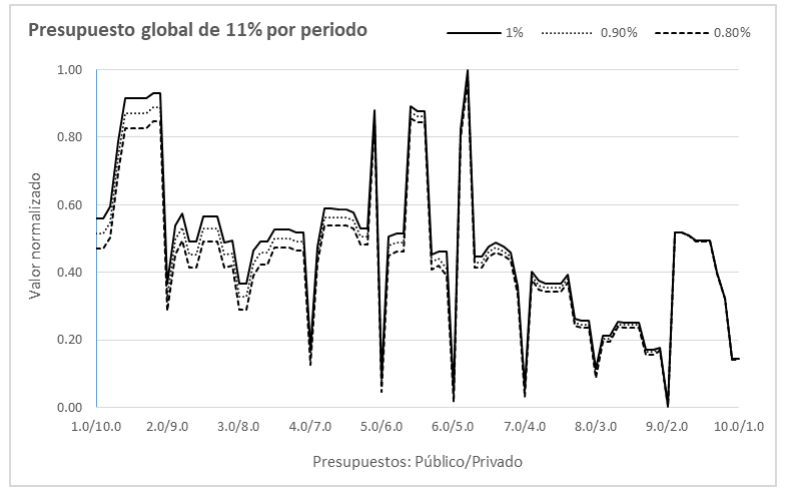

b.

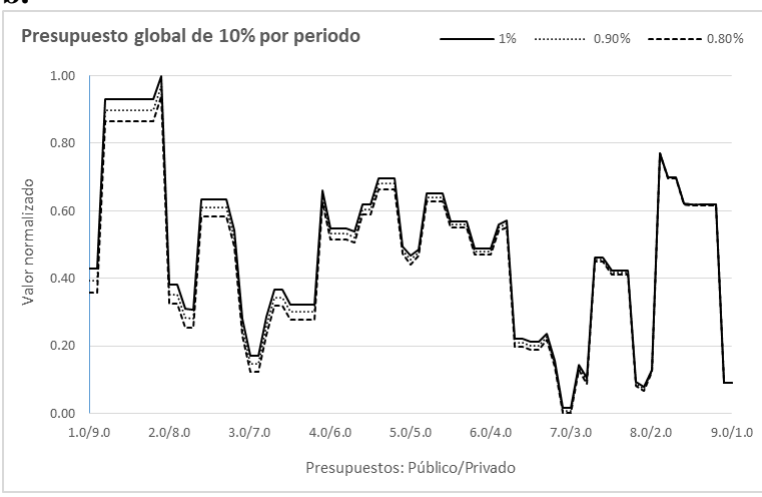

d.

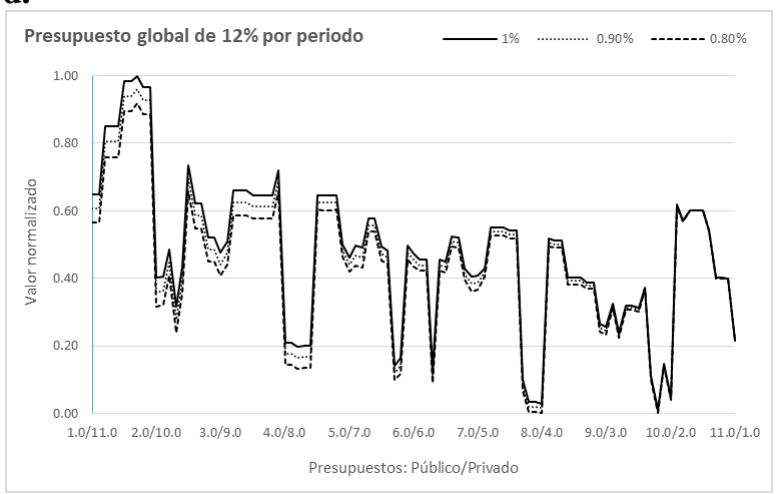

Fuente: Elaboración propia

\subsection{Discusión}

El MPLEM multi-periodo con enfoque económico desarrollado en este capítulo corresponde a la extensión del modelo presentado en el capítulo 5. Esta formulación permite analizar las 
decisiones estratégicas y tácticas en las soluciones de DERTI que satisfacen el problema combinado LI-DR intermodal. Los diferentes escenarios generados permitieron analizar los efectos en la priorización de las decisiones frente a cambios en los parámetros de costes y de presupuesto de inversión. Si bien esta formulación se validó para dos condiciones que caracterizan el problema de decisiones que se estudia, la interdependencia entre los proyectos de inversión y los múltiples presupuestos de inversión, parece adecuado considerar otras variantes para extender el trabajo en distintas direcciones Por ejemplo, definiendo nuevos tipos de decisiones tácticas y operativas que pueden incidir en la localización de las terminales intermodales, siendo interesante distinguir las capacidades y flujos para dos o más productos, así como introducir un conjunto de actividades/servicios en la terminal.

De acuerdo con la situación de la ZODES Magdalena Medio, los informes presentados para el MPLEM multi-periodo y las dos variantes sirven de guía a los tomadores de decisiones para planificar las inversiones a nivel local y regional de manera adecuada con el desarrollo del territorio. La interdependencia confirma que en un problema combinado LI-DR es aconsejable que los proyectos de inversión no se evalúen como decisiones aisladas debido a que no aportarían valor a la subregión equivalente a la inversión que suponen. Este resultado es coherente con la realidad de la subregión porque, por una parte, se garantizan la llegada del producto a la terminal y el desarrollo de las operaciones en la misma, por otra parte, contrarresta los efectos de desvalorización que deben afrontar los proyectos de infraestructura cuando se asigna la inversión de manera desarticulada. El enfoque estratégico del problema combinado condicionó la interdependencia a una relación de beneficios económicos, no obstante, ampliar la aplicación a un enfoque táctico permitiría valorar la interdependencia desde una perspectiva de los recursos físicos y materiales requeridos, en tiempo y cantidad, para la construcción de los proyectos seleccionados.

En el modelo que se consideran los múltiples presupuestos de inversión se incorporó un coste social para representar las implicaciones de la participación de actores privados en la construcción de la red intermodal, no obstante, los efectos en la solución óptima muestran cierto grado de similitud que permite intuir pocos cambios en los diseños de la red. Para esta formulación podría ser interesante analizar los efectos en la solución asumiendo distintos mecanismos de financiación y estableciendo una métrica basada en el número de unidades vehiculares que transitan en los arcos de transporte y tasas de uso en las terminales intermodales. Los beneficios en la selección de uno u otro mecanismo pueden ser compensado con indicadores de sostenibilidad social que permitan establecer un contrapeso frente a una posible aplicación desmedida de tarifas o tasas por parte del actor privado. Por otra parte, es conveniente ampliar el horizonte de planificación a la vida económica de los proyectos, incorporando también múltiples clases de decisiones o limitaciones técnicas asociadas a la construcción de la alternativa. Estas opciones permiten apreciar los periodos de inactividad ocasionados, por ejemplo, por el mantenimiento de las vías. De manera particular para la subregión que se estudia en esta tesis, a futuro podrían vincularse las decisiones de construir las vías de primera milla que se catalogaron como vías antiguas de primera milla.

De manera general es propicio que se evalúe la eficiencia de las tres formulaciones en otras redes de entrada cuyas estructuras difieran de la analizada en este documento. Además, la característica perecedera del producto exigiría incluir tiempos de viaje que podrían afectar la asignación modal y, por consiguiente, las decisiones de localización de las terminales intermodales. También parece interesante analizar los efectos de la interdependencia de 
acuerdo con las fases de construcción de los proyectos de inversión. Teniendo en cuenta que la incertidumbre es inherente tanto a la gestión de los recursos económicos como a la demanda, sería interesante ampliar los modelos con un enfoque probabilístico, o alternativamente, realizar análisis hipotéticos ante potenciales situaciones comerciales, políticas y financieras que puedan contextualizarse en escenarios bajo incertidumbre.

\subsection{Conclusiones}

En este capítulo se desarrolló y utilizó un MPLEM multi-periodo para analizar las soluciones de DERTI que satisfacen el problema combinado LI-DR intermodal. El análisis realizado ratifica la validez y pertinencia de su utilidad para la toma de decisiones en la zona de estudio, así como la aplicación del mismo en situaciones similares que se extienden a distintos países en vía de desarrollo. La utilidad del modelo se precisa en la consideración de la disponibilidad de los recursos financieros en entornos con profundas necesidades de nuevas infraestructuras para reactivar o consolidar, en torno a las cadenas de suministros más prometedoras, el sector logístico y de transporte. Los análisis de los resultados de los experimentos numéricos muestran que la disponibilidad de los recursos económicos periodo a periodo y las variaciones de los costes inciden en los proyectos priorizados. Por tanto, los efectos son notorios tanto en el diseño estratégico como en el diseño táctico de la red. El incremento en los costes de construcción y, específicamente, en los costes de transporte fluvial ocasionan incompatibilidades en los DERTI que conducen a distintas soluciones de diseño de red. La estructura de la red condiciona el diseño táctico de la misma debido a que los efectos reflejan una moderación o intensificación de los flujos a través de los arcos de las redes de transporte subyacente.

El MPLEM multi-periodo se validó para dos condiciones particulares: la interdependencia entre los proyectos de inversión y los múltiples presupuestos de inversión. Para validar cada condición se ejecutaron dos series de experimentos numéricos que consistieron en comprobar los efectos en las soluciones a través de cambios en los parámetros.

Los resultados de los experimentos numéricos realizados para el MPLEM multi-periodo con interdependencia permiten intuir que, el conjunto de restricciones incluidas a este modelo genera una ventaja en las soluciones estratégicas de DERTI, pues, para una misma disponibilidad, tiende a capturar todos los posibles incrementos de los costes en un único grupo de proyectos de inversión. En efecto se presentan disparidades puntuales, principalmente, en las alternativas que se programan cumpliendo la interdependencia. Sin embargo, la sensibilidad de la solución frente a la condición de la interdependencia conlleva a distintas configuraciones de red intermodal cuando se evalúan distintas disponibilidades de financiación. Adicionalmente, no se aprovechan los beneficios económicos ocasionados cuando la disponibilidad de financiación es reducida en cada periodo de decisión.

Los efectos de la solución óptima en la validación de los múltiples presupuestos de inversión en el en el modelo permiten inferir la importancia en la utilización del presupuesto de inversión proveniente de actores privados. Si bien los mejores resultados se logran con la mayor participación del presupuesto público, no se puede descartar que la inversión privada sea conveniente para cubrir las necesidades de infraestructura en la subregión. La definición de un presupuesto global para efectuar los análisis facilitó el reconocimiento de los límites en las participaciones para cada actor. En relación con los diseños estratégicos de la red 
intermodal, la interdependencia ejerce mayor influencia cuando se consideran múltiples presupuestos de inversión. Mientras, los efectos frente al coste social aplicado por la utilización del presupuesto privado no tienen efectos tan significativos ya que las soluciones óptimas conservan cierta semejanza. 


$$
\text { CAPítulO } 7
$$




\section{Capítulo 7}

\section{Diseño sostenible de la red de transporte intermodal: conflicto entre múltiples objetivos y preferencias de los decisores}

Resumen: en este capítulo se desarrolla y utiliza un modelo de programación lineal enteromixto (MPLEM) multi-objetivo para el problema combinado de localización de instalacionesdiseño de red (LI-DR) intermodal descrito en el capítulo 3. Los criterios sociales y medioambientales identificados para el problema de decisiones se introducen en la formulación como nuevas funciones objetivo del MPLEM presentado en el capítulo 5. El MPLEM multi-objetivo aporta al estado de la cuestión ya que los enfoques social y medioambiental han sido escasamente explorados en la literatura del campo del transporte. Los tres enfoques tratan, en conjunto, de impulsar la actividad económica y el beneficio social a la vez que se preserva el patrimonio natural. El análisis a las soluciones Pareto-óptimas se basa en determinar los efectos ante cambios en las preferencias de los decisores para cada objetivo y ante la disponibilidad de financiación como limitante del diseño. Los resultados demuestran que el conjunto de soluciones Pareto-optimas y el conflicto entre los objetivos varia con el presupuesto de financiación disponible, y que un mismo grupo de preferencias tienen efectos distintos según la disponibilidad de inversión para tomar la decisión. 


\subsection{Introducción}

El análisis de decisiones en el campo del transporte ha pasado de una toma de decisiones con un sesgado enfoque en lo económico a una toma de decisiones que contempla simultáneamente objetivos económicos, sociales y medioambientales (Yannis et al., 2020). Un nuevo proyecto de transporte no solo debe incrementar la eficiencia de las actividades económicas, también debe favorecer, entre otros, al acceso al empleo, los recursos hídricos o energéticos (Josa \& Aguado, 2019), dar cumplimiento a las normas medioambientales (Behbahani et al., 2019) y a aquellos procesos territoriales relacionados con la calidad del paisaje, la biodiversidad y el suelo (Ortega et al., 2014). Este tipo de toma de decisiones atrae el interés de diferentes actores y grupos de interés con necesidades y expectativas que entran en conflicto entre sí (Olander, 2007). Los actores privados se centran en minimizar costes, mientras que, los actores públicos buscan garantizar un impacto social y territorial favorable (Anvari \& Turkay, 2017). Además, la participación de actores públicos en la decisión avala la implicación de inversores privados (Wiegmans \& Behdani, 2018).

Las dimensiones económica, social y medioambiental difieren según el actor y grupo de interés (Cornet et al., 2018), por tanto, el conocimiento de las necesidades del problema de decisiones y el compromiso de los actores para resolverlo es determinante (Jovovic et al., 2017). Una solución basada solo en la dimensión económica no permite una comprensión completa del impacto de los proyectos y de la inversión (Rowsell et al., 2018), ya que los problemas de decisión en el campo del transporte son intrínsecamente de múltiples y contradictorios criterios (Hosseininasab et al., 2018). Las Naciones Unidas (2015) establecen que, para una toma de decisiones sostenible las opciones se deben interrelacionar, entre otros, con la erradicación de la pobreza, la reducción de todas las formas de desigualdad y la preservación del medio ambiente. Los resultados de la revisión sistemática de la literatura en dos etapas vinculas (RSL-2EV) presentados en el capítulo 4 señalan que, en los problemas combinados de localización de instalaciones-diseño de red (LI-DR) las decisiones se analizan casi en su totalidad desde el enfoque económico, minimizando costes y tiempos.

En este capítulo se desarrolla y utiliza un modelo de programación lineal entero-mixto (MPLEM) multi-objetivo para el problema combinado de localización de instalaciones-diseño de red (LI-DR) descrito en el capítulo 3. La formulación de este modelo corresponde a la versión multi-objetivo del MPLEM presentado en el capítulo 5, por lo que se introducen las funciones objetivo social y medioambiental para seleccionar los proyectos de inversión más adecuados. El modelo se resuelve con el esquema de resolución R+Gurobi (descrito en la subsección 3.5.1.) y se utiliza el método de suma ponderada (MSP) para obtener el conjunto de soluciones Pareto-óptimas. El análisis a la solución de diseño sostenible de la red de transporte intermodal (DSRTI) se basa en determinar los efectos ante cambios en las preferencias de los decisores y la disponibilidad de financiación.

El capítulo está organizado de la siguiente manera. En la sección 7.2. se presenta una revisión de la literatura sobre los criterios sociales y medioambientales utilizados en problemas de decisión relacionados con los problemas combinados LI-DR. En la sección 7.3. se formulan las funciones objetivo social y medioambiental para el MPLEM multi-objetivo. En la sección 7.4. se presenta el análisis de las soluciones obtenidas al optimizar de manera individual el modelo con las funciones objetivo social y medioambiental, así como el grado de conflicto existente entre los tres objetivos. En la sección 7.5. se analizan las soluciones Pareto-óptimas 
para el DSRTI. Las secciones 7.6. y 7.7. presentan, respectivamente, la discusión y las conclusiones de este capítulo.

\subsection{Revisión de la literatura}

En los problemas de localización de instalaciones y en los problemas de diseño de red los criterios sociales y medioambientales propuestos se han estudiado para la toma de decisiones en ámbito de la cadena de transporte (Litman, 2007) y en la gestión de la cadena de suministro (Messmann et al., 2020). La evaluación de proyectos de infraestructura en estos ámbitos implica que los tomadores de decisiones definan criterios y preferencias para comparar el impacto de los proyectos (Cornet et al., 2018) para priorizar la inversión (Leibowicz, 2020). En la subsección 7.2.1. se presentan los criterios sociales, y en la subsección 7.2.2. los criterios medioambientales.

\subsubsection{Criterios sociales}

Bubicz et al. (2019) exponen que la dimensión social de la sostenibilidad debe evaluarse desde cuatro aspectos: condiciones laborales, sociedad, derechos humanos y responsabilidad del producto. En lo que respecta a la selección de proyectos de transporte, la densidad poblacional, el empleo, la accesibilidad y los servicios comunitarios básicos son aspectos importantes (Jeon \& Amekudzi, 2005). No obstante, los criterios sociales han tenido una atención limitada en la literatura del diseño de redes intermodales (Moreno-Camacho et al., 2019), porque, en general, son criterios de difícil consenso y cuantificación para ser incorporados en los modelos de programación matemática (Xiao et al., 2017). En la Tabla 7.1. se listan los criterios sociales identificados en la literatura.

Las condiciones laborales representan el grupo de criterios más utilizado, siendo el empleo creado el de mayor aplicación, generalmente, maximizando los puestos de trabajo creados al abrir/construir una instalación o infraestructura de transporte. En un número limitado de modelos se ha propuesto incorporar un criterio relacionado con la destrucción de los puestos de trabajo que se ocasiona por el cierre de las instalaciones. Arampantzi \& Minis (2017) incorporan este criterio junto con los accidentes y muertes de los empleados en el cumplimiento de sus funciones. Por otra parte, existe un creciente interés de las organizaciones por las implicaciones en los empleados y la sociedad. Anvari \& Turkay (2017), pensando en el bienestar de los empleados, consideran la disponibilidad de servicios médicos y educativos y el nivel de seguridad en las inmediaciones donde se evalúa la localización de las instalaciones. Otros criterios, pero de poca concurrencia, consideran las condiciones de las zonas de potencial localización, por ejemplo, la accesibilidad (Konstantinidou et al., 2019), el desempleo e inmigración (Tsao \& Thanh, 2019) y el desarrollo regional (Sherafati et al., 2019).

Para el MPLEM multi-objetivo que se formula en la sección 7.3. se incorporan nuevos criterios sociales que se pueden situar en el grupo de las características de la zona de estudio, estos son: pobreza multidimensional, inclusión social y pérdidas de producto. 
Tabla 7.1. Criterios sociales ${ }^{41}$

\begin{tabular}{|c|c|c|}
\hline Criterios & & Referencias \\
\hline \multirow[t]{2}{*}{ 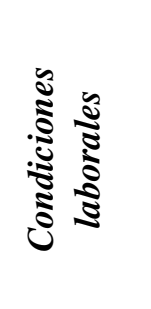 } & Empleo creado & $\begin{array}{l}\text { Allaoui et al. (2018), Anvari \& Turkay (2017), Arampantzi \& Minis } \\
\text { (2017), Bal \& Satoglu (2018), Mota et al. (2015), Pourjavad \& } \\
\text { Mayorga (2019), Fathollahi-Fard et al. (2018), Moheb-Alizadeh \& } \\
\text { Handfield (2019), Rahimi et al. (2019), Sahebjamnia et al. (2018), } \\
\text { Vafaei et al. (2020) }\end{array}$ \\
\hline & Empleo destruido & Allaoui et al. (2018), Arampantzi \& Minis (2017) \\
\hline \multirow{5}{*}{ 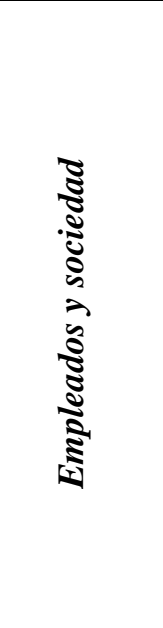 } & $\begin{array}{l}\text { Instalaciones } \\
\text { médicas }\end{array}$ & Anvari \& Turkay (2017) \\
\hline & $\begin{array}{l}\text { Instalaciones } \\
\text { educativas }\end{array}$ & Anvari \& Turkay (2017) \\
\hline & $\begin{array}{l}\text { Accidentes y } \\
\text { muertes }\end{array}$ & $\begin{array}{l}\text { Arampantzi \& Minis (2017), Fathollahi-Fard et al. (2018), Ko et al. } \\
\text { (2019), Moheb-Alizadeh \& Handfield (2019), Sahebjamnia et al. } \\
\text { (2018) }\end{array}$ \\
\hline & $\begin{array}{l}\text { Satisfacción de la } \\
\text { demanda }\end{array}$ & Anvari \& Turkay (2017), Rad \& Nahavandi (2018) \\
\hline & $\begin{array}{l}\text { Desempleo y } \\
\text { migración }\end{array}$ & Tsao \& Thanh (2019) \\
\hline \multirow{3}{*}{ 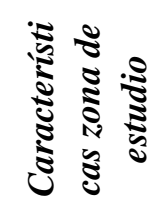 } & Seguridad y riesgo & Anvari \& Turkay (2017), Akgün \& Erdal (2019) \\
\hline & Desarrollo regional & Anvari \& Turkay (2017), Sherafati et al. (2019) \\
\hline & Accesibilidad & Konstantinidou et al. (2019) \\
\hline
\end{tabular}

\subsubsection{Criterios medioambientales}

La Tabla 7.2. lista los criterios medioambientales utilizados en la literatura. Entre estos, las emisiones contaminantes se emplean con más frecuencia. Tsao \& Thanh (2019) diseñan una red de puertos secos en un entorno incierto en el cual la dimensión medioambiental minimiza las emisiones relacionadas con la apertura y operación del puerto y el transporte. Por su parte, Anvari \& Turkay (2017) intentan equilibrar la localización de instalaciones entre zonas desarrolladas y no desarrolladas, considerando el consumo y contaminación del agua. Miret et al. (2016) utilizan el método Ecocostes para incorporar el agotamiento de los recursos naturales, el efecto sobre los ecosistemas, la salud humana y el calentamiento global. En general, los criterios propuestos tienen una importante implicación medioambiental basada en la estrategia empresarial y en el cumplimiento de normas establecidas por las entidades reguladoras y convenios internacionales.

Litman (2007) menciona que el ruido, la contaminación del aire y del agua, el cambio climático, la pérdida de hábitats y el agotamiento de los recursos no renovables son aspectos relevantes para valorar la viabilidad en la construcción de proyectos de infraestructura de

\footnotetext{
${ }^{41}$ En el anexo B se compila un listado más amplio de los criterios de optimización de sostenibilidad social y medioambiental que se han identificado en la literatura de los problemas de decisiones.
} 
transporte. Phillips et al. (2020) explica que los efectos pueden extenderse hasta $1 \mathrm{~km}$ del perímetro del proyecto y recomiendan que la planificación estratégica de la infraestructura de transporte sea compatible con principios de conservación (García-Montero et al., 2010). El transporte intermodal es reconocido como una práctica estratégica para reducir las emisiones en el transporte, pese a ello aún existe un vacío en la literatura que permita profundizar y diversificar los criterios utilizados en los modelos de programación (Kumar \& Anbanandam, 2019). Por lo tanto, es importante explorar otros criterios relacionados con la planificación del territorio y la gestión de los recursos naturales de especies y hábitats.

Para el MPLEM multi-objetivo que se formula en la sección 7.3. se incorporan dos criterios medioambientales centrados en asociar la intervención y transformación del territorio a las decisiones estratégicas. Los criterios son: la preservación de la biodiversidad y el suelo agrícola productivo.

Tabla 7.2. Criterios medioambientales ${ }^{42}$

\begin{tabular}{|c|c|c|}
\hline Criterios & & Referencias \\
\hline \multirow{9}{*}{ 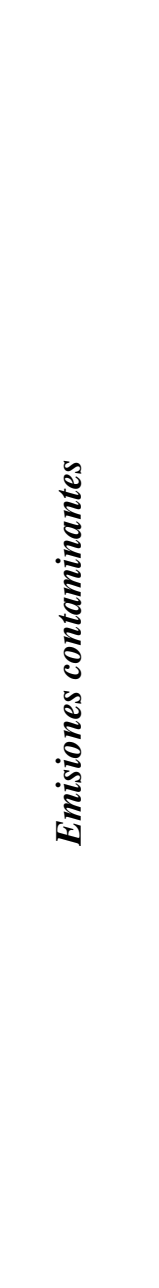 } & $\begin{array}{l}\text { Apertura/construcción } \\
\text { de la instalación }\end{array}$ & $\begin{array}{l}\text { Allaoui et al. (2018), Tsao \& Thanh (2019), Arampantzi \& Minis } \\
\text { (2017), Mogale et al. (2020), Rahimi et al. (2019), Vafaei et al. } \\
\text { (2020) }\end{array}$ \\
\hline & $\begin{array}{l}\text { Operación en la } \\
\text { instalación }\end{array}$ & $\begin{array}{l}\text { Tsao \& Thanh (2019), Mogale et al. (2020), Moheb-Alizadeh \& } \\
\text { Handfield (2019), Mohammed et al. (2019), Zhou et al. (2018) }\end{array}$ \\
\hline & $\begin{array}{l}\text { Procesamiento de } \\
\text { materias primas }\end{array}$ & Allaoui et al. (2018), Arampantzi \& Minis (2017) \\
\hline & Fabricación & $\begin{array}{l}\text { Allaoui et al. (2018), Arampantzi \& Minis (2017), Pourjavad \& } \\
\text { Mayorga (2019), Rad \& Nahavandi (2018), Rahimi et al. (2019) }\end{array}$ \\
\hline & Transporte & $\begin{array}{l}\text { Allaoui et al. (2018), Ameknassi et al. (2016), Anvari \& Turkay } \\
\text { (2017), Arampantzi \& Minis (2017), Bal \& Satoglu (2018), } \\
\text { Pourjavad \& Mayorga (2019), Mohammed et al. (2019), Tsao \& } \\
\text { Thanh (2019), Guo et al. (2018), Ko et al. (2019), Maiyar \& Thakkar } \\
\text { (2019), Mogale et al. (2020), Moheb-Alizadeh \& Handfield (2019), } \\
\text { Rad \& Nahavandi (2018), Rahimi et al. (2019), Rohmer et al. (2019), } \\
\text { Vafaei et al. (2020), Zhou et al. (2018) }\end{array}$ \\
\hline & Almacenamiento & $\begin{array}{l}\text { Ameknassi et al. (2016), Arampantzi \& Minis (2017), Mogale et al. } \\
(2020)\end{array}$ \\
\hline & $\begin{array}{l}\text { Reprocesamiento de } \\
\text { productos }\end{array}$ & Ameknassi et al. (2016), Rad \& Nahavandi (2018) \\
\hline & Consumo de energía & Allaoui et al. (2018), Arampantzi \& Minis (2017) \\
\hline & $\begin{array}{l}\text { Disposición de } \\
\text { producto }\end{array}$ & Pourjavad \& Mayorga (2019) \\
\hline$\nabla \infty \infty$ & Consumo & Allaoui et al. (2018), Anvari \& Turkay (2017), Guo et al. (2018) \\
\hline
\end{tabular}

${ }^{42}$ Ibíd 41. 


\begin{tabular}{|c|c|c|}
\hline & Contaminación & Anvari \& Turkay (2017) \\
\hline \multirow{2}{*}{$\frac{\mathbb{8}}{\tilde{5}}$} & $\begin{array}{l}\text { Producción de } \\
\text { alimentos }\end{array}$ & Rohmer et al. (2019) \\
\hline & $\begin{array}{l}\text { Construcción de las } \\
\text { instalaciones }\end{array}$ & Anvari \& Turkay (2017) \\
\hline \multirow{2}{*}{ 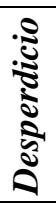 } & Manejo del producto & Maiyar \& Thakkar (2019) \\
\hline & $\begin{array}{l}\text { En la cadena de } \\
\text { suministro }\end{array}$ & Anvari \& Turkay (2017) \\
\hline \multirow{4}{*}{ 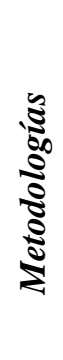 } & Ciclo de vida (LCA) & Sahebjamnia et al. (2018) \\
\hline & $\begin{array}{l}\text { Análisis de inventario } \\
\text { LCA y ReCiPe } 2008\end{array}$ & Mota, et al. (2015) \\
\hline & Método de Ecocostes & Miret et al. (2016) \\
\hline & $\begin{array}{l}\text { Modelo de pluma } \\
\text { gaussiana }\end{array}$ & Assadipour et al. (2016) \\
\hline
\end{tabular}

\subsection{Propuesta del MPLEM multi-objetivo}

En esta sección se formulan las funciones objetivo social y medioambiental para el MPLEM multi-objetivo. Las funciones se construyen atendiendo a los criterios de optimización social y medioambientales descritos en el capítulo 3. Para la formulación del modelo matemático se emplean los supuestos básicos (i) a (vi) y (ix) definidos en la subsección 5.3.1. Por tanto, en la subsección 7.3.1. se enuncia la notación utilizada en el MPLEM multi-objetivo, y en las subsecciones 7.3.2. y 7.3.3. se presentan los criterios de optimización que integran las funciones objetivo con enfoque social y medioambiental, respectivamente.

\subsubsection{Notación del MPLEM multi-objetivo}

Para la formulación del MPLEM multi-objetivo se mantiene la estructura de la red de entrada como un grafo dirigido $G=(N, A)$ y se complementa la notación descrita en la subsección 5.3.2. En la Tabla 7.3. se presenta la nomenclatura complementaria.

\section{Tabla 7.3. Notación complementaria para el MPLEM multi-objetivo}

\footnotetext{
Parámetros para el objetivo social

$U T_{k, a} \quad$ Número de trabajadores necesarios para construir de la alternativa $k \in K$ del $\operatorname{arco} a \in A_{1} \cup A_{4}$

$H a b_{i} \quad$ Número de habitantes en el nodo de origen $i \in I$

$E s t_{i} \quad$ Número de estudiantes en primaria y secundaria en el nodo de origen $i \in I$

$M u j_{i} \quad$ Número de mujeres líderes en las áreas de cultivo agrícola del nodo de origen $i \in I$

$\mathrm{PobM}_{i}$ Índice de pobreza multidimensional en el nodo de origen $i \in I$
} 


\section{Parámetros para el objetivo medioambiental}

$H_{e c C_{i}}$ Número de hectáreas cosechadas en el nodo de origen $i \in I$

THecC Número total de hectáreas cosechadas en la subregión

$P \cos _{i} \quad$ Cantidad de producto potencialmente cosechable en el nodo de origen $i \in I$

$E s p_{i} \quad$ Número de especies endémicas y en extinción en el área de influencia del nodo de origen $i \in I$

$\operatorname{Proy}_{i, a}$ Número proyectos $a \in A_{1} \cup A_{4}$ que impactan en el área de influencia del nodo de origen $i \in I$

\subsubsection{Objetivo social}

Para construir el MPLEM multi-objetivo se propone una función objetivo con enfoque social que busca maximizar el beneficio que se genera a la población y los cultivadores de la región con los proyectos de inversión que se seleccionen. La función se compone de tres criterios: pobreza multidimensional (PM), inclusión social (IS) y pérdidas de producto (PP), y se representa con la estructura matemática que se muestra en (7.1). Los componentes de cada uno de los criterios mencionados se desagregan y explican a continuación.

$$
\max P M+I S+P P
$$

\subsubsection{Pobreza multidimensional}

La pobreza es una métrica clave para el direccionamiento de las políticas y el desarrollo de las regiones (Santos \& Villatoro, 2018). En la toma de decisiones del campo del transporte parece ideal que las decisiones se apoyen en el uso de métricas relacionadas con la pobreza dado que se tiene efectos tanto en el ámbito social como el desarrollo económico (Litman, 2019). El criterio PM se calcula de acuerdo con la formula (7.2). Este criterio se inclina a favorecer la inversión a partir del índice de pobreza multidimensional $\operatorname{PobM}_{i}$, el número de habitantes $\mathrm{Hab}$ y el número de proyectos potenciales $\operatorname{Proy}_{i}$ que impactan en el nodo cada nodo de origen $i \in$ I.

$$
\sum_{i \in I} \sum_{k \in K} \sum_{a \in A_{1} \cup A_{4}}\left(\left(\text { PobM }_{i} \cdot \text { Hab }_{i}\right) / \text { Proy }_{i, a}\right) \cdot \beta_{k, a}
$$

\subsubsection{Inclusión social}

La inclusión social, aunque se ha entendido como un objetivo para la operacionalización política, en la práctica se centra en la participación de la población en entornos sociales y comunitarios que mejoran la calidad de vida (Cordier et al., 2017). En otras palabras, significa que todos pueden participar de manera adecuada en actividades y oportunidades importantes, tales como: acceso a servicios, educación, empleo y toma de decisiones (Litman, 2019). El criterio IS se define por el número de personas que se pueden beneficiar con la construcción de los proyectos de inversión, considerando entre sus componentes la población estudiantil 
(7.3), las mujeres líderes en las áreas de cultivo agrícola (7.4) y la oportunidad laboral (7.5). En los dos primeros componentes, el número de estudiantes $P E_{i}$ y las mujeres líderes $M L_{i}$ se

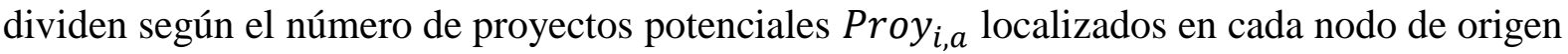
$i \in I$. El tercer componente se relaciona con la creación de trabajo $U T_{k, a}$ a partir de las alternativas de capacidad/vial que se selecionan.

Es importante mencionar que, la distinción de las cultivadoras exige un análisis profundo en relación con la estructura familiar y la toma de decisiones intrafamiliar para evitar efectos contraproducentes en niñas, niños y adolescentes. Meemken \& Qaim (2018) exponen que en la mayoría de los países en vía de desarrollo a menudo son los hombres, considerados jefes de hogar, quienes disponen y controlan los ingresos generados de la producción y comercialización de cultivos; por su parte, las mujeres solo tienen una mínima intervención en la decisión con el control para gastos menores. Por lo tanto, la inclusión de las mujeres líderes en el MPLEM multi-objetivo se contempla como una forma de visibilizar y respaldar el desarrollo de la actividad agrícola, ya que en la región se promueven iniciativas que propenden por el fortalecimiento del sector a partir de la participación activa de la mujer.

Población estudiantil

$$
\sum_{i \in I} \sum_{k \in K} \sum_{a \in A_{1} \cup A_{4}}\left({ }^{E s t_{i}} / \operatorname{Proy}_{i, a}\right) \cdot \beta_{k, a}
$$

Mujeres líderes

$$
\sum_{i \in I} \sum_{k \in K} \sum_{a \in A_{1} \cup A_{4}}\left({ }^{M u j_{i}} / \text { Proy }_{i, a}\right) \cdot \beta_{k, a}
$$

\section{Oportunidad laboral}

$$
\sum_{k \in K} \sum_{a \in A_{1} \cup A_{4}} U T_{k, a} \cdot \beta_{k, a}
$$

\subsubsection{Pérdidas de producto}

Pese a existir una gran necesidad para reducir la pérdida de alimentos, en el ámbito de la cadena de suministro agrícola el interés por estudiarlas es aún reducido (Shukla \& Jharkharia, 2013). Algunos estudios han evaluado las causas de la pérdida de la cosecha agrícola, por ejemplo, Kasso \& Bekele (2018) identifican que la distancia del mercado, el precio, la falta y condiciones de medios de transporte adecuados son aspectos que inciden en las pérdidas del producto. Las mayores contribuciones conciernen a la planificación operativa del transporte (Nourbakhsh et al., 2016) y a la pérdida de calidad del producto para su posterior procesamiento (Sun \& Fan, 2020). En lo referente a las pérdidas de producto en los orígenes no suelen considerarse, pero se reconoce que pueden llegar a ser significativamente altas (Xie et al., 2014). 
En la subsección 5.4.4. se analizó el impacto de las pérdidas de producto en los nodos de origen para el problema combinado LI-DR intermodal que se estudia en esta tesis. Aunque no sé contabilizaron los efectos económicos, si se reconoció la profunda repercusión entre los cultivadores y los posibles desequilibrios en las soluciones de DERTI, más aún, en aquellas soluciones donde el nodo de origen queda excluido del diseño. Los tomadores de decisiones y los responsables en la formulación de políticas deben analizar la compensación entre los aspectos económicos y las disparidades en el territorio para planificar inversiones que promuevan el crecimiento de las regiones (Lall et al., 2014). En este sentido se propone el criterio de PP (7.6), el cual se define a través de una relación de las hectáreas cosechadas en cada nodo de origen $\mathrm{HecC}_{i}$ con las hectáreas totales cosechadas en toda la subregión $\mathrm{THecC}$. Esta relación se modula según las decisiones de pérdidas en cada nodo de origen $W_{i}$.

$$
\sum_{i \in I}\left(\mathrm{HecC}_{i} /_{\text {TotHecC }}\right) \cdot W_{i}
$$

\subsubsection{Objetivo medioambiental}

Para el MPLEM multi-objetivo se propone una función objetivo con enfoque medioambiental que minimiza el impacto ocasionado por la intervención y transformación del territorio. El impacto se mide a través de los criterios de preservación de la biodiversidad (PB) y el suelo agrícola productivo (SAP). La estructura matemática que se muestra en (7.7) representa esta función. Los componentes de cada uno de los criterios mencionados se desagregan a continuación.

$$
\min P B+S A P
$$

\subsubsection{1. $\quad$ Preservación de la biodiversidad}

La inversión en infraestructura reduce los costes de transporte, mejora la productividad y atrae otros factores que fomentan el crecimiento económico de las regiones (Witte et al., 2014). Sin embargo, desde una perspectiva de medioambiente, la infraestructura de transporte también conduce a una fragmentación y pérdida continuada de los ecosistemas (Ortega, 2014), por lo que la planificación de nuevos trazados en la red de carreteras debe apuntar hacia una gestión sostenible (Álvarez-Miranda et al., 2019). La zona de estudio cuenta en su área de influencia con un patrimonio natural caracterizado por una biodiversidad, distinguida a nivel nacional y mundial, en la que habitan especies endémicas y amenazadas. Con el criterio de PB (7.8) se pretende localizar $\beta_{k, a}$ el menor número de proyectos de inversión Proy $_{i, a}$ en aquellos nodos de origen $i \in I$ donde el número de especies endémicas y amenazadas $E s p_{i}$ se alto.

$$
\sum_{i \in I} \sum_{k \in K} \sum_{a \in A_{1} \cup A_{4}} E s p_{i} \cdot \operatorname{Proy}_{i, a} \cdot \beta_{k, a}
$$




\subsubsection{2. $\quad$ Suelo agrícola productivo}

Los cultivos agrícolas son una fuente significativa de degradación del suelo debido al uso de fertilizantes y pesticidas (Oren, 2004). Esta degradación impacta directamente en la disminución o pérdida de los atributos físicos, químicos (nutrientes), biológicos (reservas de carbono) y ecológicos (funciones de los ecosistemas y purificación del agua) del suelo (Lal, 2015). Con el criterio de suelo agrícola productivo (7.9) se busca favorecer la selección y localización controlada de los proyectos acorde con el nivel de desarrollo de la agricultura en cada nodo de origen. El criterio SAP se define por la diferencia entre la cantidad de producto potencialmente cosechable $P \cos _{i}$ y el producto disponible $P_{i}$ que minimiza el número de proyectos potenciales $\operatorname{Proy}_{i, a}$ localizados en el área de influencia del nodo de origen $i \in I$.

$$
\sum_{i \in I} \sum_{k \in K} \sum_{a \in A_{1} \cup A_{4}}\left(P \cos _{i}-P_{i}\right) \cdot \operatorname{Proy}_{i, a} \cdot \beta_{k, a}
$$

\subsection{Optimización individual de las funciones objetivo social y medioambiental}

Para apreciar con más claridad los efectos de los funciones social y medioambiental en las soluciones de DSRTI del problema combinado LI-DR intermodal, primero se analizan las soluciones obtenidas al optimizar de manera individual el MPLEM mono-periodo para ambas funciones, y luego, se analizan los resultados del MPLEM multi-objetivo. En esta sección se informan los resultados de la optimización individual. Para llevar a cabo este análisis se resuelven 1200 instancias, aplicando los valores detallados en la Tabla 5.2. ${ }^{43}$, con el esquema de resolución expuesto en la subsección 3.5.1. Todos los resultados presentados se centran en los obtenidos para Cap2. En la subsección 7.4.1. se analizan los efectos de la disponibilidad del presupuesto de inversión en las soluciones óptimas. Además, dado que los requerimientos mínimos y máximos de inversión para los objetivos social y medioambiental difieren de los requerimientos del objetivo económico, se trata de determinar un rango de inversión factible para resolver el MPLEM multi-objetivo. En la subsección 7.4.2. se precisa el grado de conflicto existente entre los tres objetivos.

\subsubsection{Análisis de la solución óptima social y medioambiental}

Las Figuras 7.1. y 7.2. revelan las soluciones óptimas para los enfoques social y medioambiental, respectivamente, para distintos presupuestos y niveles de demanda. Las curvas son construidas a partir de cada instancia resuelta. Cada punto de la curva representa la proporción del valor obtenido en ese punto respecto al óptimo que ofrece la situación más favorable para cada objetivo. Los espacios sombreados indican el intervalo de presupuestos en el que no es factible obtener una solución óptima, y a su vez determinan la mínima inversión requerida para localizar al menos un proyecto de inversión.

Desde el enfoque social, los resultados sugieren un requerimiento de inversión equivalente a P100 en los tres niveles de demanda. Estos resultados se corresponden con el sentido de

\footnotetext{
${ }^{43}$ La Tabla se muestra en la subsección 5.4.
} 
maximización de la función, pues, son afines con la selección de todos los proyectos de inversión para brindar el mayor beneficio a la población. En contraparte, desde el enfoque medioambiental se identifica un requerimiento máximo de inversión que varía levemente con el nivel de demanda. Para Dem1 el este requerimiento se sitúa en P12, para Dem2 en P16 y para Dem3 en P9. Aunque la Figura muestra una prolongación del óptimo hasta P100, se debe entender que no se agota el presupuesto de inversión cuyas disponibilidades sean superiores a las disponibilidades mencionadas. Por tanto, más recursos económicos no suponen la localización de más proyectos dado que el enfoque medioambiental apunta a transformar el territorio sobre la base de la preservación del entorno natural y no a favorecer el flujo de productos.

Ambos enfoques imponen nuevos requerimientos mínimos y máximos de inversión, que difieren significativamente de los requerimientos de inversión identificados con el enfoque económico, por lo que en el siguiente apartado se trata de determinar un rango de inversión factible para resolver el MPLEM multi-objetivo.

Figura 7.1. Soluciones óptimas en el beneficio social con Cap2

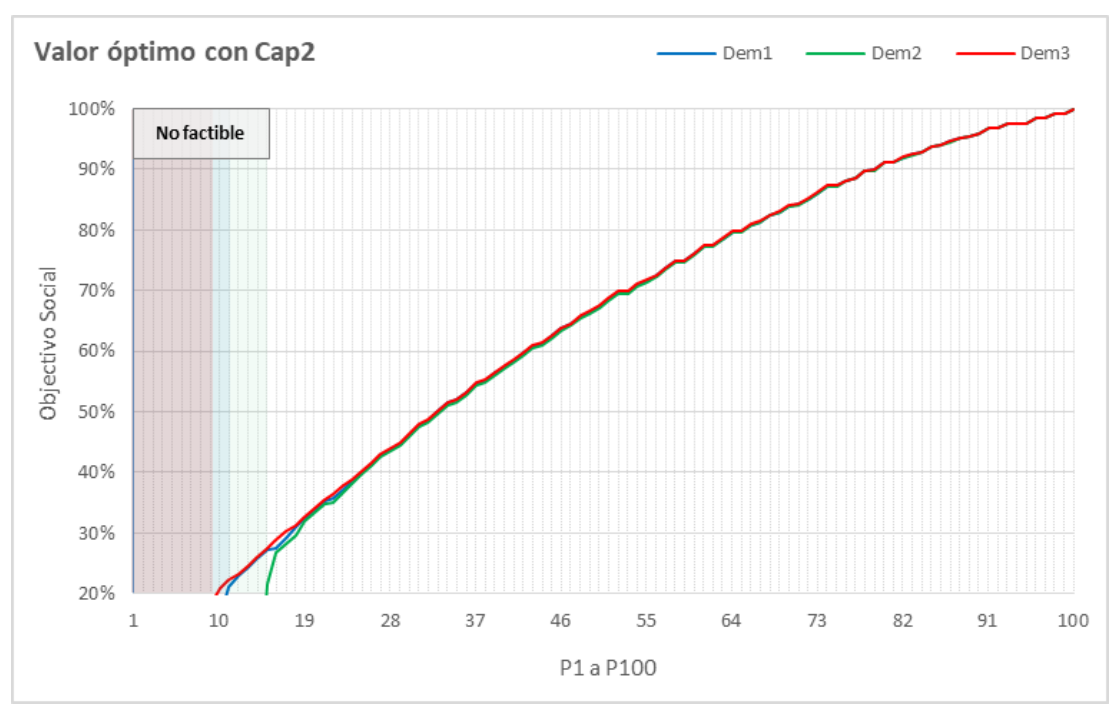

Fuente: Elaboración propia 
Figura 7.2. Soluciones óptimas en el impacto medioambiental con Cap2

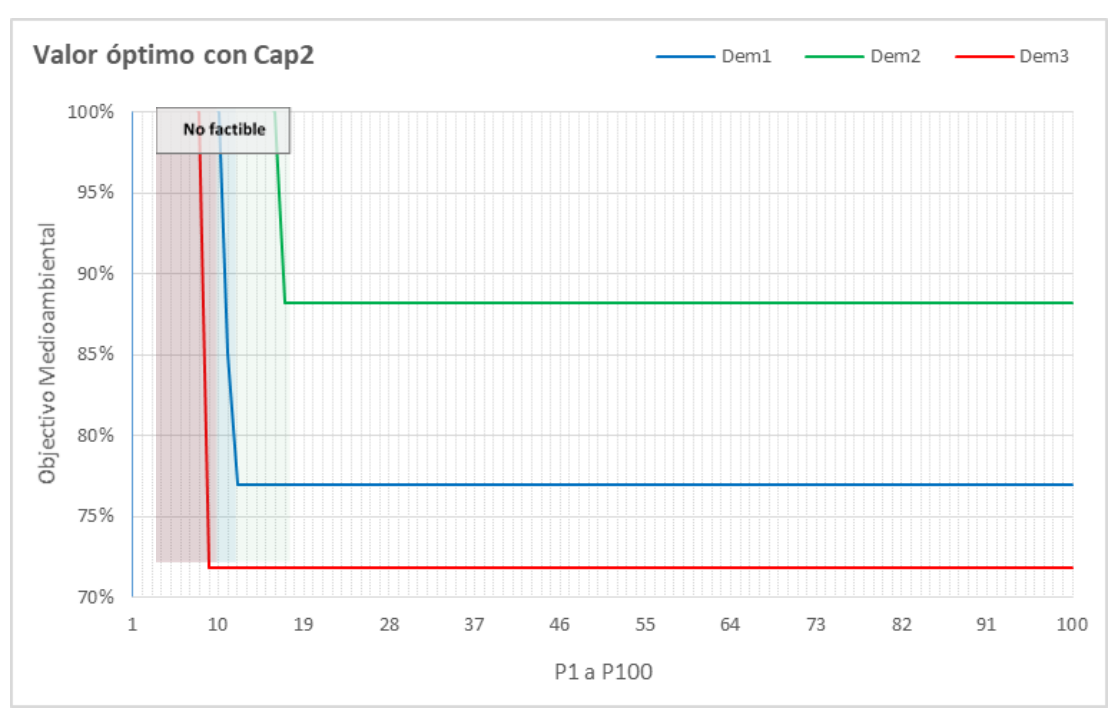

Fuente: Elaboración propia

\subsubsection{1. $\quad$ Límites de inversión admisibles para los tres objetivos}

En la Tabla 7.4. se muestran los requerimientos de inversión mínimos y máximos para obtener soluciones factibles según el enfoque de cada objetivo. Para los tres objetivos el mínimo requerimiento depende del nivel de demanda. Entre los objetivos económico y medioambiental el máximo requerimiento tiene una diferencia sustancial, por lo que es una primera aproximación de la incompatibilidad existente entre los objetivos. Tal incompatibilidad es más pronunciada entre los objetivos social y medioambiental, ya que el máximo requerimiento de inversión social se sitúa en P100 para todos los niveles de demanda.

La suposición definida en la formulación de la restricción de presupuesto permite asumir que los tomadores de decisión tienen como propósito asignar de manera adecuada los recursos económicos y no en conocer que tan distantes están de la inversión óptima o del impacto más favorable. Sin embargo, ante el modelo multi-objetivo y el gran desafío de cómo plantear las preferencias de cada objetivo, cobra importancia entender esa distancia para definir requerimientos de inversión que permitan establecer los límites admisibles para obtener las soluciones de DSRTI.

Tabla 7.4. Requerimientos de inversión por objetivo de optimización con Cap2

\begin{tabular}{lccc|ccc|ccc}
\hline \multirow{2}{*}{ PInv $^{t}$} & \multicolumn{8}{c|}{ Objetivos de optimización } \\
\cline { 2 - 10 } & \multicolumn{3}{c|}{ Económico } & \multicolumn{3}{c}{ Social } & \multicolumn{3}{c}{ Medioambiental } \\
\hline Mínimo & P10 & P15 & P8 & P10 & P15 & P8 & P10 & P15 & P8 \\
\hline Máximo & P59 & P61 & P51 & P100 & P100 & P100 & P12 & P16 & P9 \\
\hline
\end{tabular}

Con la finalidad de dar continuidad a los resultados que se presentan en esta sección, se determinan los límites de inversión admisibles para el nivel de demanda Dem1. Las Figuras 
7.3., 7.4. y 7.5. representan, por pares de objetivos, el impacto del óptimo admisible de un objetivo si se apropia como límite de inversión la disponibilidad del objetivo opuesto. Si bien los tomadores de decisiones pueden definir otras estrategias, para este trabajo se consideró aproximar los límites máximo y mínimo de inversión conforme a los objetivos económico y medioambiental, respectivamente.

Comparando los límites máximos de inversión de los objetivos económico y social, Figura 7.3., se identifica una amplia brecha de disponibilidades (P60 a P100) que no tienen efectos económicos para el problema combinado LI-DR intermodal, pero que si tienen efectos favorables en el objetivo social. Puesto que en la realidad no es posible financiar todos los proyectos de inversión parece razonable abandonar el límite social de P100 y asumir un nuevo límite para analizar las soluciones de DSRTI. En este sentido, se revisa el impacto social para la disponibilidad P59 y se asume el valor de $74.90 \%$ como la nueva solución óptima más favorable para el objetivo social.

Por su parte, en la comparación de los objetivos económico y medioambiental, Figura 7.4., la situación es inversa pareciendo necesario trasladar el mínimo requerimiento de P10 a P12. Con este cambio además de mantenerse el mejor resultado del objetivo medioambiental, se mejora el mínimo impacto económico. En la comparación de los objetivos medioambiental y social, Figura 7.5., se muestra el beneficio para el objetivo social que se logra con asumir la disponibilidad P12 como el mínimo requerimiento de inversión. En definitiva, el intervalo de inversión para el DSRTI se define entre P12 y P59.

Figura 7.3. Límites de inversión y óptimos admisibles para los objetivos económico y social

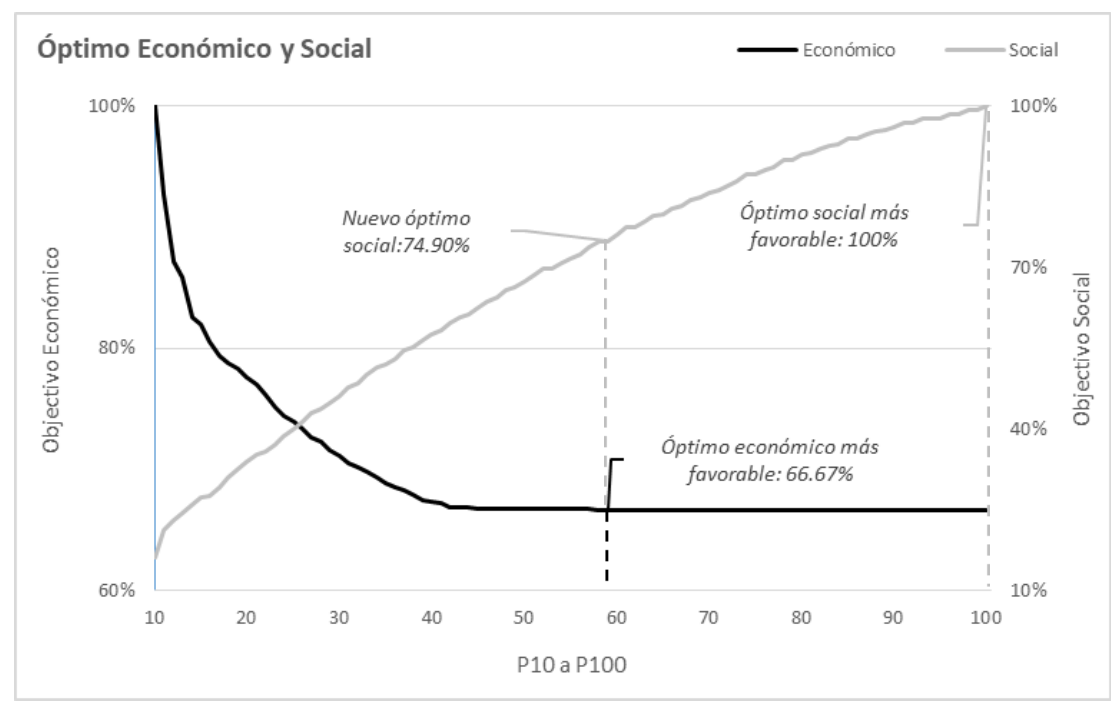

Fuente: Elaboración propia 
Figura 7.4. Límites de inversión y óptimos admisibles para los objetivos económico y medioambiental

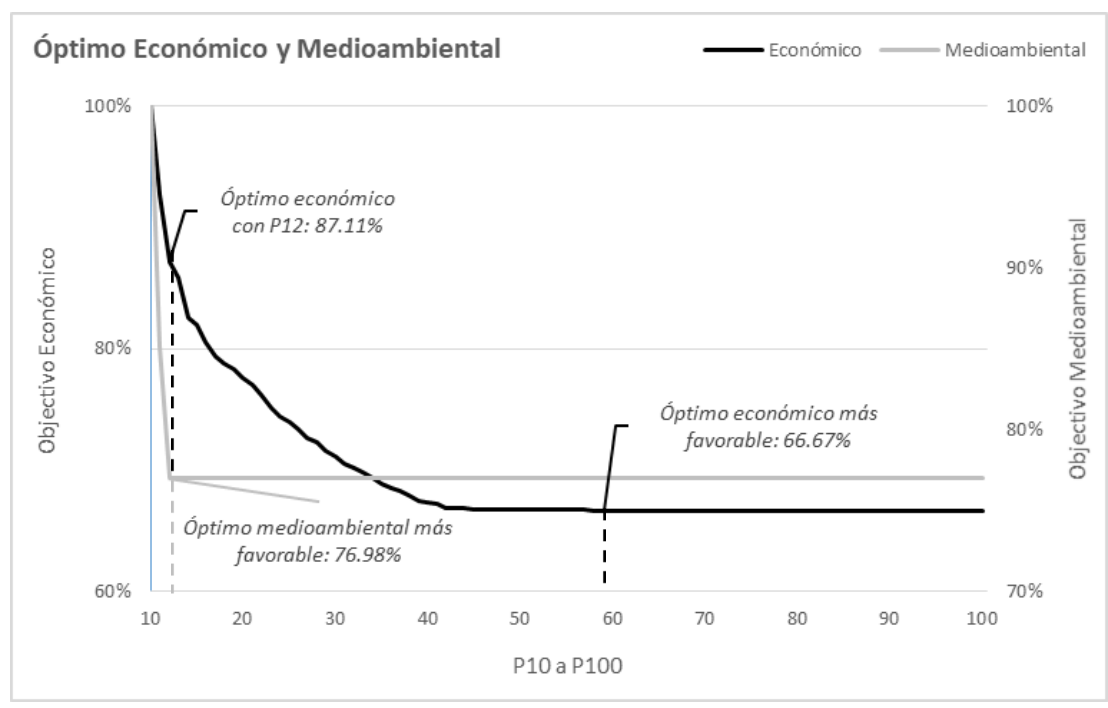

Fuente: Elaboración propia

Figura 7.5. Límites de inversión y óptimos admisibles para los objetivos medioambiental y social

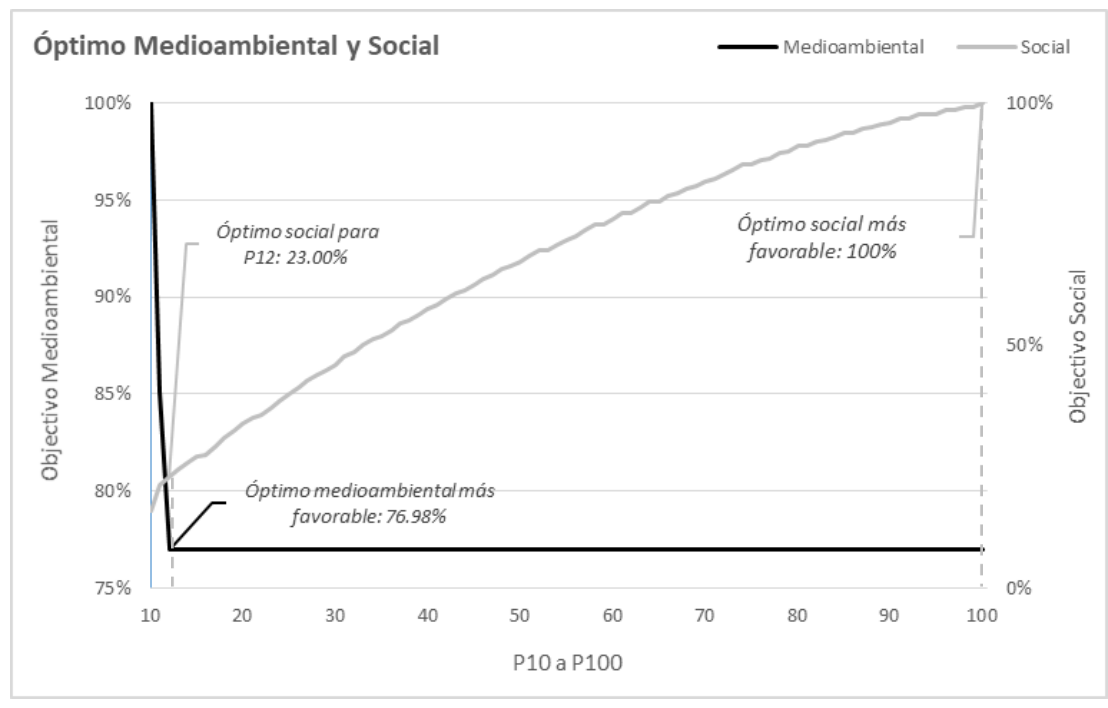

Fuente: Elaboración propia

\subsubsection{Grado de conflicto entre los objetivos}

Las Figuras 7.6. y 7.7. muestran las soluciones de diseño económico de la red de transporte intermodal (DERTI), diseño social de la red de transporte intermodal (DSORTI) y diseño medioambiental de la red de transporte intermodal (DMRTI) obtenidas al optimizar cada función objetivo con disponibilidades de P12 y P25, respectivamente. En cada diseño se indica el grado de conflicto de la solución para los otros dos objetivos. El conflicto entre los objetivos permite medir la variación porcentual del impacto de un objetivo en una solución de otro objetivo en relación con su óptimo (Anvari \& Turkay, 2017). El conflicto entre los objetivos se analiza a continuación. 
Con la disponibilidad P12, Figura 7.6., la solución de DERTI tiene un impacto social que se ubica en $23.41 \%$ por debajo del óptimo social para esta misma disponibilidad, así mismo, tiene un impacto medioambiental que excede en $92.49 \%$ el óptimo más favorable para este objetivo. La interpretación de este conflicto desde la estructura física del diseño muestra ciertas diferencias en la selección y localización de los proyectos de primera milla, la exclusión de los nodos de origen y el uso de las redes subyacentes para la asignación de los flujos. Por ejemplo, en el DERTI se reduce el uso de la red de carretera y se fortalece la red fluvial, por el contrario, en el DMRTI se procura el uso de las redes subyacentes de tal modo que se utiliza toda la red ferroviaria proporcionada como entrada al modelo.

Con la disponibilidad P25, Figura 7.7., el grado de conflicto se intensifica entre unos objetivos y se atenúa entre otros. La solución de DERTI puede generar un impacto social que dista en $19.41 \%$ del óptimo social más favorable, pero incrementa el impacto medioambiental hasta $289.9 \%$ por encima de su óptimo. Aunque el conflicto se atenúa entre los objetivos económico y social, este resultado no supone la convergencia en los proyectos seleccionados. El DERTI induce la construcción de ambos tipos de proyectos de inversión, mientras que, el DSORTI procura la construcción sesgada a los proyectos intermodales. La solución de DMRTI se mantiene igual a la solución con P12. Es resultado se debe entender a la luz del enfoque del objetivo medioambiental, el cual se centra en la transformación el territorio sobre la base de la preservación del entorno natural, por lo que las soluciones de DMRTI se mantienen sin agotar el presupuesto de inversión.

Si bien la implementación de la solución de DERTI puede suponer diferencias físicas con la solución de DSORTI, el conflicto se va atenuando a medida que se tiene más disponibilidad de inversión. Se debe entender que este efecto no es reciproco si se considera la implementación de la solución de DSORTI. La implementación del DMRTI intensifica el grado de conflicto con los objetivos económico y social cuando se incrementa el presupuesto. Dado que el grado de conflicto entre los objetivos varia con el presupuesto disponible, es apropiado que se consideren estas variaciones para asignar las preferencias en el diseño sostenible de la red intermodal. 
Figura 7.6. Grado de conflicto entre los objetivos con presupuesto P12

Diseño de la Red Intermodal

$\$$

$0 \%$

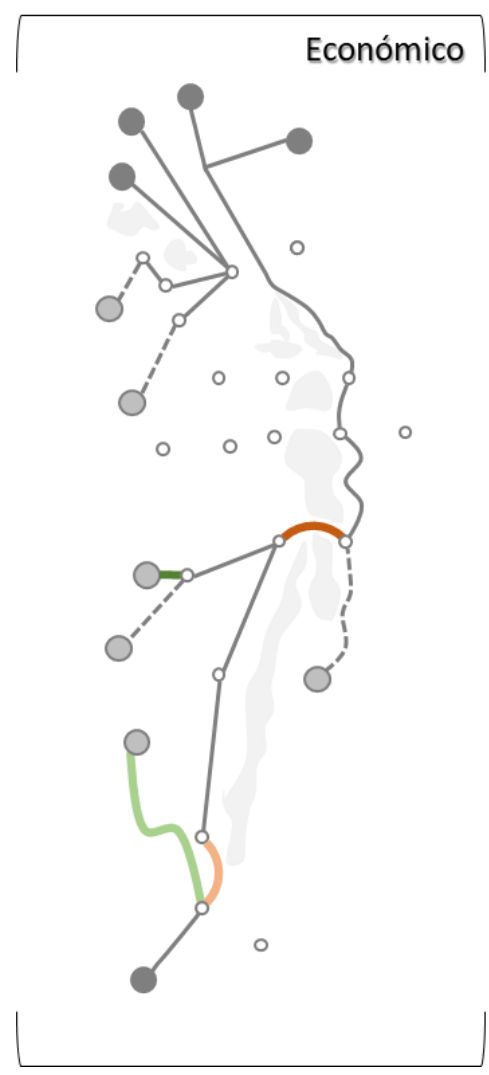

Grado de conflicto

Obj. Social: $-23.41 \%$

Obj. Medioambiental: $+92.49 \%$

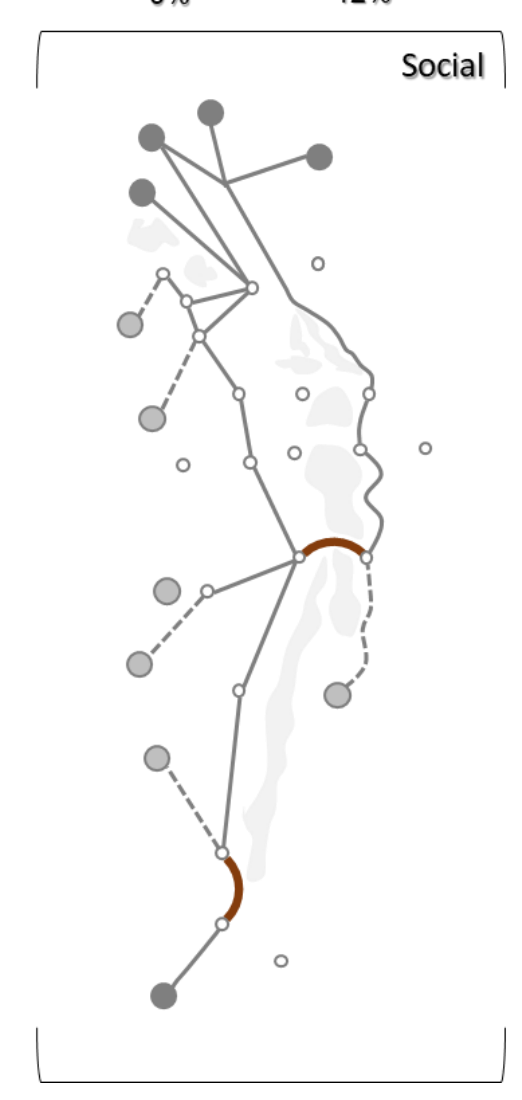

Grado de conflicto

Obj. Económico: $+16.77 \%$

Obj. Medioambiental: $+94.87 \%$

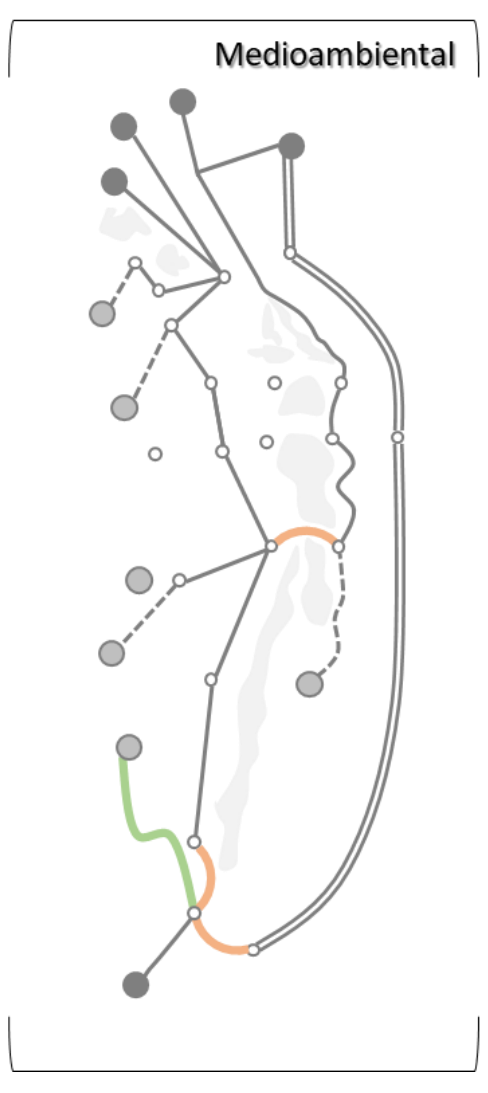

Grado de conflicto

Obj. Económico: $+12.06 \%$

Obj. Social: $-49.24 \%$

Fuente: Elaboración propia 


\section{Figura 7.7. Grado de conflicto entre los objetivos con presupuesto P25}

\section{Diseño de la Red Intermodal}

$\$$

$0 \%$

$12 \%$

$25 \%$

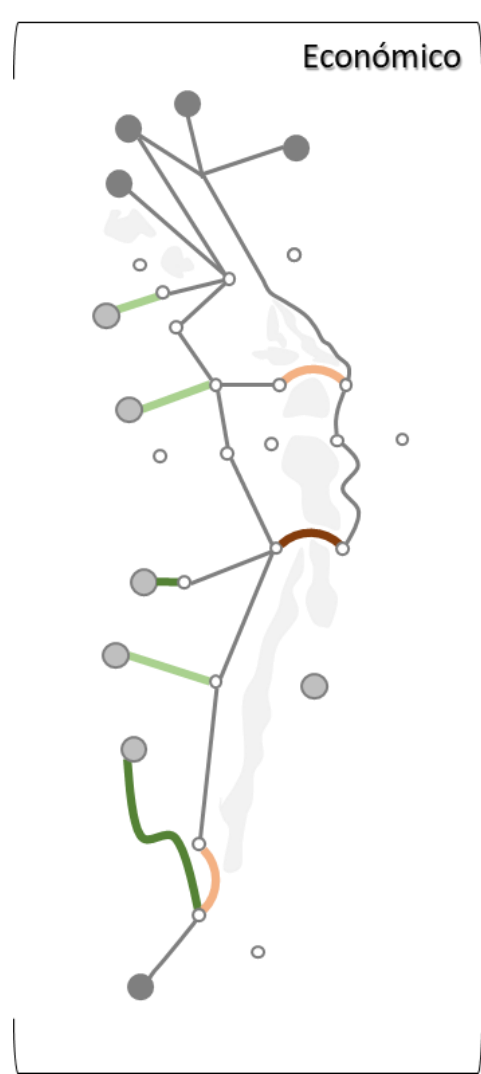

Grado de conflicto

Obj. Social: $-19.41 \%$

Obj. Medioambiental: $+289.9 \%$

Social

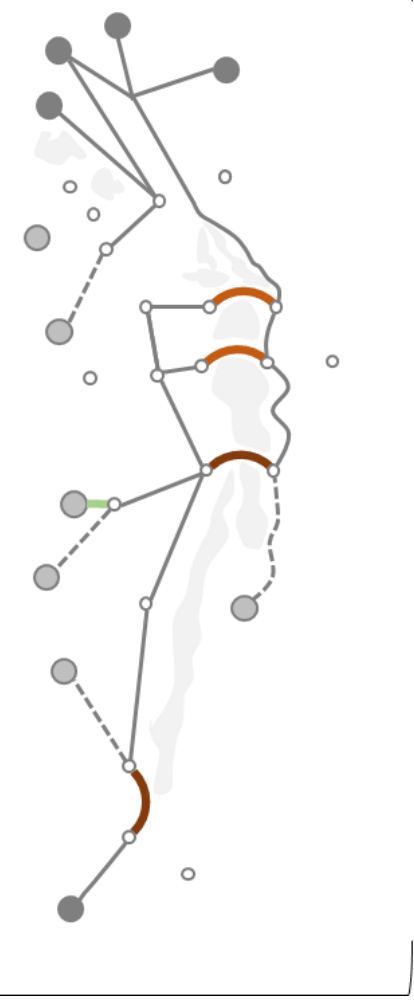

Grado de conflicto

Obj. Económico: $+32.65 \%$

Obj. Medioambiental: $+562.3 \%$

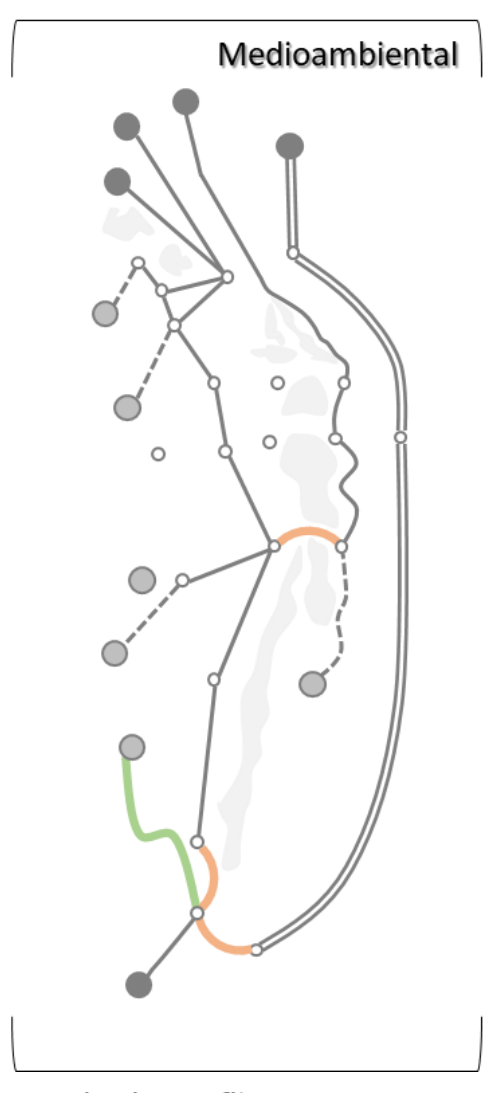

Grado de conflicto

Obj. Económico: $+32.13 \%$

Obj. Social: $-70.86 \%$

Fuente: Elaboración propia 


\subsection{DSRTI para el problema combinado LI-DR intermodal}

En esta sección se analizan los resultados del DSRTI del MPLEM multi-objetivo propuesto para el problema combinado LI-DR intermodal. La concurrencia de las tres funciones objetivos propuestas en la formulación y la limitación de financiación sugieren un análisis direccionado a las estrategias de preferencias por objetivo (vector de pesos) para lograr soluciones Paretoóptimas que satisfagan, en distinta medida, cada objetivo del modelo. Para llevar a cabo este análisis, en la subsección 7.5.1. se construyen las matrices de pago y fronteras de Pareto con el propósito de entender la incompatibilidad conjunta de los tres objetivos frente a la asignación de las preferencias de los decisores. En la subsección 7.5.2. se analiza las soluciones de DSRTI asignando distintas preferencias (vectores de pesos) a los objetivos en el momento de resolver el MPLEM multi-objetivo.

\subsubsection{Estrategias de Preferencias: Matriz de pago y frontera de Pareto}

La matriz de pago es una matriz adimensional que se construye para determinar los puntos extremos del espacio de soluciones Pareto-óptimas de una programación multi-objetivo (Marler \& Arora, 2010). Los valores de la matriz se calculan, primero, a partir de la solución óptima de cada objetivo resuelto de manera individual, luego, con cada una de esas soluciones se calcula el resultado para los otros objetivos y se identifican los valores ideales y nadir (Romero \& Rehman, 2003). El valor ideal corresponde al óptimo de cada objetivo y el valor nadir se define por el peor valor que puede obtener un objetivo en la matriz de pago. Para definir los valores ideal y nadir es recomendable normalizar los valores. Para la normalización se aplica la función de transformación (7.10) utilizada por Marler \& Arora (2004). $F_{i}^{o}$ representa el valor ideal y $F_{i}^{\text {max }}$ el valor nadir.

$$
F_{i}^{\text {trans }}=\frac{F_{i}(X)-F_{i}^{o}}{F_{i}^{\text {max }}-F_{i}^{o}}
$$

De acuerdo con los límites de inversión admisibles definidos en la subsección 7.4.1. se puede construir una matriz de pago por cada disponibilidad de financiación del intervalo P12 a P59, no obstante, en esta subsección solo se analizan los resultados de P12, P25, P45 y P5944. La Tabla 7.5. muestra las matrices de pago normalizadas para los cuatro presupuestos mencionados. Los valores ideales se encuentran en la diagonal principal de cada matriz y los valores nadir son iguales a 1. A partir de las matrices se representan las fronteras de Pareto en la Figura 7.8.

Con las matrices de pago se puede determinar que la asignación de la mayor preferencia al objetivo económico lleva a una correspondencia positiva con el objetivo social a medida que se mejora la disponibilidad de presupuesto, ya que la incompatibilidad se reduce de 0.476 a 0.105 . Mientras que, en la situación opuesta, con la mayor preferencia al objetivo social se ocasiona un incremento sustancial de los costes, que se agudiza con bajos presupuestos. En la

\footnotetext{
${ }^{44}$ Aunque los análisis anteriores y posteriores que se informan en este capítulo refieren a las disponibilidades P12 y P25, se incorpora de manera excepcional en esta subsección los presupuesto P45 y P59 para precisar el análisis de las estrategias de preferencias con altas disponibilidades de inversión.
} 
relación de los objetivos económico y medioambiental, la asignación de mayor preferencia al primero supone efectos variados en el segundo, en una escala de 0.516 a 0.975 , que no se corresponden con la disponibilidad de presupuesto. Por el contrario, una gran preferencia al objetivo medioambiental produce efectos desfavorables al objetivo económico que se incrementan cuando se dispone más inversión. La relación de los objetivos social y medioambiental enmarca el mayor conflicto en ambas direcciones para todos los presupuestos, por lo que la asignación de las preferencias siempre producirá efectos adversos en el objetivo contrario.

Las fronteras de Pareto muestran los conjuntos de soluciones Pareto-óptimas que se pueden obtener con todas las posibles combinaciones de preferencias ${ }^{45}$. Es importante mencionar que en un problema multi-objetivo los valores ideales y nadir son soluciones inalcanzables e inviables, donde toda la preferencia se asigna a un único objetivo (Romero \& Rehman, 2003). Las cuatro fronteras de Pareto tienen una forma similar con pliegues que generan distintas secciones. Las secciones de la frontera que se ubican hacia los extremos implican grandes compensaciones ocasionadas por la asignación casi que total de la preferencia a un único objetivo, en cambio, en las secciones centrales se considera que los objetivos tienen preferencias con proporciones aproximadas que provocarían efectos moderados en los resultados de cada uno. El presupuesto disponible influye en el área que conforma algunas de estas secciones. En las más cercanas a los valores ideales de los objetivos económico y social se aprecia la reducción del área en conflicto al incrementar la disponibilidad, en tanto que, en las más cercana al objetivo medioambiental la incompatibilidad se intensifica.

Dado que el efecto de la preferencia asignada a cada objetivo puede cambiar según la disponibilidad de presupuesto, es importante definir distintas estrategias de preferencias. Por ejemplo, con el presupuesto P12 se podría considerar la mayor y media preferencia para los objetivos económico y social, respectivamente, y una menor preferencia para el objetivo medioambiental. La limitada disponibilidad de inversión en sí misma condiciona la trasformación del territorio ya que el número de proyectos localizables es reducido, por tanto, se pueden aprovechar los efectos en la minimización de los costes o en la maximización del beneficio social para el DSRTI. Por el contrario, con presupuestos que tienden al límite admisible P59, y entendiendo la concordancia que va logrando el beneficio social respecto a la reducción de los costes cuando se incrementa el presupuesto, es posible destinar la mayor preferencia al objetivo medioambiental y preferencias intermedias a los objetivos económico y social. De esta manera se controla la excesiva localización de proyectos.

Tabla 7.5. Matrices de pago normalizadas para P12, P25, P45 y P59

\begin{tabular}{llc|c|c}
\hline \multirow{2}{*}{$\boldsymbol{P I n v}^{t}$} & \multicolumn{3}{c}{ Valores ideales y nadir normalizados } \\
\cline { 3 - 5 } & & Económico & Social & Medioambiental \\
\hline \multirow{2}{*}{$\mathbf{P 1 2}$} & Económico & $\mathbf{0 . 0 0 0}$ & 0.476 & 0.975 \\
\cline { 2 - 5 } & Social & 1.000 & $\mathbf{0 . 0 0 0}$ & 1.000 \\
\cline { 2 - 5 } & &
\end{tabular}

\footnotetext{
${ }^{45}$ Las fronteras de Pareto se construyen con los nueve valores obtenidos de cada matriz de pago, es posible que en la aplicación de un método más riguroso la figura presente leves modificaciones en algunas de sus secciones.
} 


\begin{tabular}{lll|l|l} 
& Medioambiental & 0.719 & 1.000 & $\mathbf{0 . 0 0 0}$ \\
\hline \multirow{2}{*}{$\mathbf{P 2 5}$} & Económico & $\mathbf{0 . 0 0 0}$ & 0.274 & 0.516 \\
\cline { 2 - 5 } & Social & 1.000 & $\mathbf{0 . 0 0 0}$ & 1.000 \\
\cline { 2 - 5 } & Medioambiental & 0.984 & 1.000 & $\mathbf{0 . 0 0 0}$ \\
\hline \multirow{2}{*}{$\mathbf{P 4 5}$} & Económico & $\mathbf{0 . 0 0 0}$ & 0.231 & 0.729 \\
\cline { 2 - 5 } & Social & 0.832 & $\mathbf{0 . 0 0 0}$ & 1.000 \\
\cline { 2 - 5 } & Medioambiental & 1.000 & 1.000 & $\mathbf{0 . 0 0 0}$ \\
\hline & Económico & $\mathbf{0 . 0 0 0}$ & 0.105 & 0.941 \\
\cline { 2 - 5 } & Social & 0.999 & $\mathbf{0 . 0 0 0}$ & 1.000 \\
\cline { 2 - 5 } & Medioambiental & 1.000 & 1.000 & $\mathbf{0 . 0 0 0}$ \\
\hline
\end{tabular}

Figura 7.8. Frontera de Pareto para P12, P25, P45 y P59

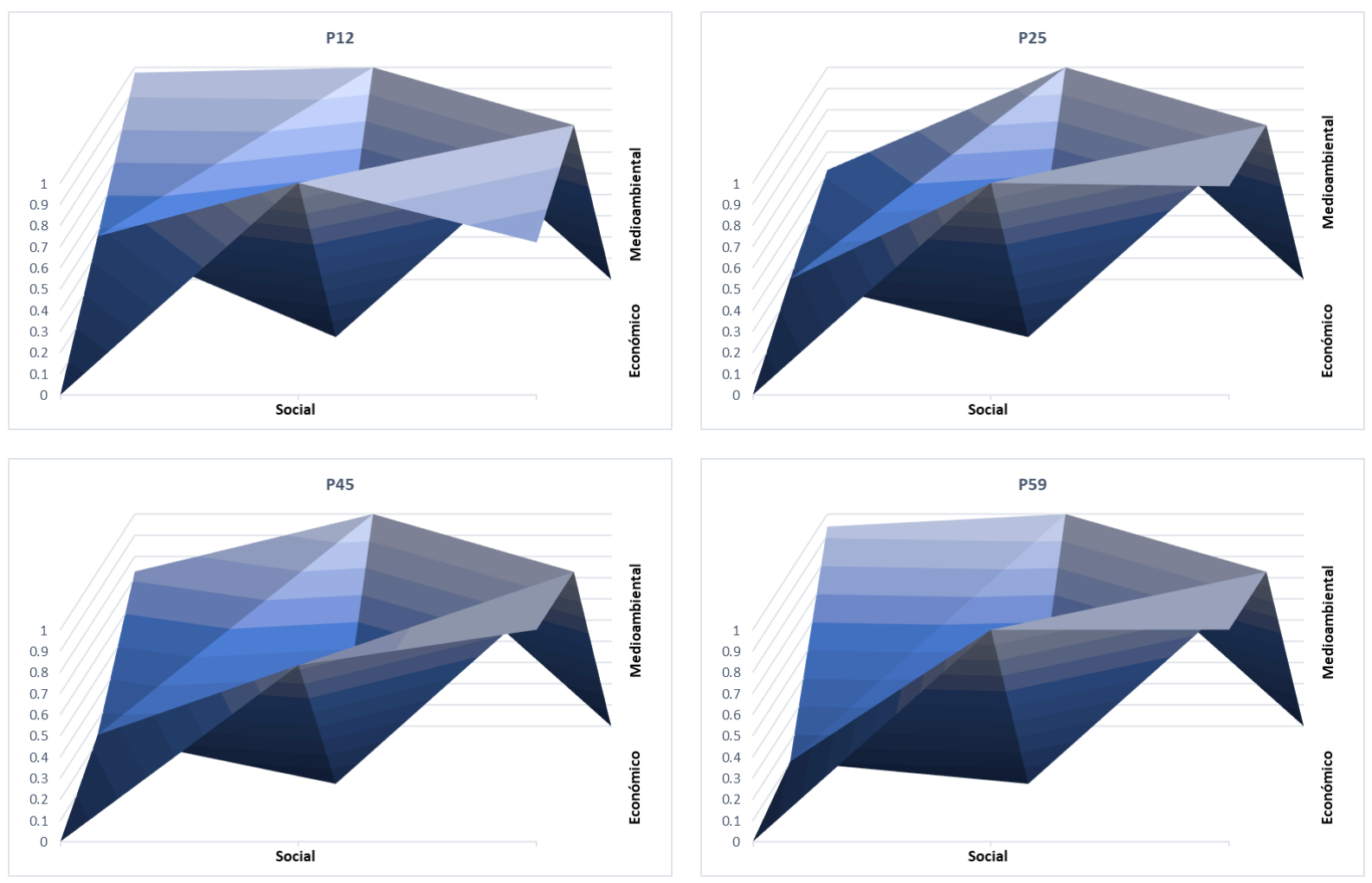

Fuente: Elaboración propia

\subsubsection{Soluciones Pareto-óptimas del MPLEM multi-objetivo}

En esta subsección se analizan las soluciones Pareto-óptimas del DSRTI asignando distintas preferencias (vectores de pesos) a cada objetivo del MPLEM multi-objetivo. Las soluciones Pareto-óptimas, también conocidas como eficientes o no dominadas, son todas aquellas soluciones para las cuales no es posible mejorar el valor de ninguna función objetivo sin empeorar los valores de al menos una de las otras funciones (Mansouri et al., 2015). En una 
solución Pareto-óptima el concepto de óptimo se reemplaza por el de eficiencia de Pareto (Mavrotas, 2009), pues, se proporcionan valores aceptables para cada objetivo de acuerdo con las preferencias asignadas (Farahani et al., 2010). Para la resolución de una programación multi-objetivo se requiere de la utilización de métodos especializados (Cho, Wang, et al., 2017). En esta tesis, además de aplicar el procedimiento de resolución descrito en la subsección 3.5.1., se utiliza el MSP ${ }^{46}$.

El MSP se destaca porque permite modelar y resolver problemas multi-objetivo con enfoque de objetivo único (Eskandarpour, 2015), además, es de gran utilidad para generar soluciones Pareto-óptimas cuando se tienen más de dos funciones objetivo (Marler \& Arora, 2010). La aplicación del MSP consiste en construir una función objetivo global a partir de todas las funciones objetivo definidas para el modelo de programación. La función global proporciona una solución de la frontera de Pareto por cada iteración (Mogale et al., 2018). Para obtener una solución se asignan pesos a cada objetivo. Los pesos representan las preferencias de los tomadores de decisiones y la suma de estos es igual a 1 (Mavrotas, 2009). Es importante que los valores de los objetivos se normalicen para que adquieran valores adimensionales entre 0 y 1 (Esteso et al., 2018).

Para obtener una solución Pareto-óptima de DSRTI se minimiza la función global (7.11) sujeta a las restricciones (5.2) a $(5.11)^{47}$. En la función global, $f^{\prime}(1), f^{\prime}(2)$ y $f^{\prime}(3)$ corresponden a los valores normalizados de $f(1),-f(2)$ y $f(3)$. La función $f(1)$ representa el objetivo económico propuesto en el capítulo 5. Las funciones $-f(2)$ y $f(3)$ representan, respectivamente, los objetivos social y medioambiental propuestos en la sección 7.3. La función del objetivo social se multiplica por -1 para que coincida con el sentido de minimización de la función global (Bolouri et al., 2018). Los parámetros $\omega 1, \omega 2$ y $\omega 3$ identifican los vectores de pesos asignados a cada objetivo. Estos pesos representan las preferencias de los tomadores de decisiones.

$$
\text { fglobal }=\min \left\{\omega 1 \cdot f^{\prime}(1)+\omega 2 \cdot f^{\prime}(2)+\omega 3 \cdot f^{\prime}(3)\right\}, \quad[\omega 1+\omega 2+\omega 3=1]
$$

Para el análisis de las soluciones Pareto-óptimas de DSRTI se asignan 6 vectores de pesos $[\omega 1+\omega 2+\omega 3=1]$ distintos. En las Tablas 7.6. y 7.7. se presentan los resultados que se obtenidos al limitar el presupuesto de inversión con P12 y P25. Estos resultados revelan que las soluciones globales menos ventajosas son aquellas donde el peso más alto se asigna al objetivo social o medioambiental, por ejemplo, las soluciones 2 y 3 tanto en P12 como en P25. En la medida que los pesos asignados se van equilibrando la soluciones reflejan valores globales próximos entre sí, como sucede con las soluciones 4 y 5 para P12. Mientras, con P25 la asignación equilibrada de los pesos produce el comportamiento contrario.

\footnotetext{
${ }^{46} \mathrm{El}$ MSP es un método que, se considera de tipo escalar por la forma como se reformulan los objetivos, y de tipo a priori por el momento en el que los tomadores de decisiones expresan las preferencias.

${ }^{47}$ Estas restricciones se encuentran en la subsección 5.3.3.
} 
Tabla 7.6. Soluciones Pareto-óptimas de DSRTI con P12

\begin{tabular}{c|cc|cc|cc|c}
\hline Solución & $\boldsymbol{\omega 1}$ & Económico & $\mathbf{\omega 2}$ & Social & $\mathbf{\omega 3}$ & Medioambiental & fglobal \\
\hline 1 & 0.8 & 0.0107 & 0.1 & 0.6059 & 0.1 & 0.4604 & 1.0770 \\
\hline 2 & 0.1 & 0.8826 & 0.8 & 0.0006 & 0.1 & 0.9986 & 1.8819 \\
\hline 3 & 0.1 & 0.6321 & 0.1 & 0.9992 & 0.8 & 0.0116 & 1.6429 \\
\hline 4 & 0.6 & 0.3581 & 0.3 & 0.1463 & 0.1 & 0.9378 & 1.4421 \\
\hline 5 & 0.3 & 0.5084 & 0.1 & 0.7274 & 0.6 & 0.1116 & 1.3473 \\
\hline 6 & 0.1 & 0.8826 & 0.6 & 0.0149 & 0.3 & 0.9880 & 1.8855 \\
\hline
\end{tabular}

Tabla 7.7. Soluciones Pareto-óptimas de DSRTI con P25

\begin{tabular}{c|cc|cc|cc|c}
\hline Solución & $\boldsymbol{\omega 1}$ & Económico & $\mathbf{\omega 2}$ & Social & $\mathbf{\omega 3}$ & Medioambiental & fglobal \\
\hline 1 & 0.8 & 0.1322 & 0.1 & 0.1772 & 0.1 & 0.4107 & 0.7202 \\
\hline 2 & 0.1 & 0.4109 & 0.8 & 0.0181 & 0.1 & 0.6867 & 1.1157 \\
\hline 3 & 0.1 & 0.5311 & 0.1 & 0.5744 & 0.8 & 0.0510 & 1.1565 \\
\hline 4 & 0.6 & 0.3323 & 0.3 & 0.1046 & 0.1 & 0.4426 & 0.8795 \\
\hline 5 & 0.3 & 0.5136 & 0.1 & 0.5815 & 0.6 & 0.0533 & 1.1483 \\
\hline 6 & 0.1 & 0.4906 & 0.6 & 0.0761 & 0.3 & 0.3804 & 0.9472 \\
\hline
\end{tabular}

La Figura 7.9. representa las soluciones Pareto-óptimas para el DSRTI presentadas en las tablas 7.6. y 7.7. Los proyectos de inversión $\mathrm{C} 1$ a C8, $\mathrm{H} 1$ y $\mathrm{H} 2$ son la infraestructura de primera milla. Los proyectos TI1 a TI4 son terminales intermodales carretera-fluvial, TI5 es carreteraferroviaria, y TI6 y TI7 son fluvial-ferroviaria. Los diseños de la Figura revelan la influencia de las preferencias de los decisores para seleccionar ciertos proyectos de inversión y en la característica de la alternativa vial o de capacidad. Con P12, pese a que el número de proyectos seleccionables es limitado por la baja disponibilidad de inversión, se pueden distinguir cinco diseños de red ya que las soluciones 4 y 6 solo difieren en las características de capacidad de las redes intermodales. Estos diseños impulsan el transporte fluvial y la intermodalidad carretera-fluvial. Por su parte, con P25 se expande la viabilidad de localización de los proyectos de primera milla por carretera y de la terminal intermodal TI1. Se distinguen cuatro diseños de red y dos soluciones en las que difieren las características viales de primera milla o de capacidad de las terminales intermodales, como en las soluciones de red 3 y 5 , y las soluciones de red 4 y 6.

En concordancia con las estrategias de preferencia definidas en la subsección anterior, la asignación del mayor y medio peso al objetivo económico y social produce con P12 (solución 4) la tercera mejor solución Pareto-óptima con el vector de peso [0.6, 0.3, 0.1]. Sin embargo, esta solución genera resultados más satisfactorios para el objetivo social que para el económico. Una explicación a este comportamiento se fundamenta en la divergencia en los proyectos localizables desde cada dimensión de sostenibilidad, como se puede ver en la Figura 7.9. Con 
más presupuesto (P25) este mismo vector de pesos ofrece un resultado global atractivo, pero pueden surgir dudas respecto al impacto en cada objetivo, especialmente en el medioambiental. Para abordarlo se debe entender que con más presupuesto se amplía el número de proyectos localizables y con ello las posibilidades de moderar el impacto medioambiental, por lo tanto, no es necesario que el resultado sea totalmente desfavorable. Comparando los diseños sostenibles de las soluciones 4 y 6 para P25 se esclarece esta interpretación.

Figura 7.9. Soluciones estratégicas de DSRTI para distintos vectores de peso y presupuesto

\begin{tabular}{|c|c|c|c|c|c|c|c|c|c|c|c|c|c|c|c|c|c|c|}
\hline \multirow{3}{*}{\multicolumn{2}{|c|}{ Solución }} & \multicolumn{17}{|c|}{ Proyectos de Construcción } \\
\hline & & \multicolumn{8}{|c|}{ Carretera } & \multicolumn{2}{|c|}{ Fluvial } & \multicolumn{7}{|c|}{ Terminales Intermodales } \\
\hline & & $\mathrm{C} 1$ & $\mathrm{C} 2$ & $\mathrm{C} 3$ & $\mathrm{C} 4$ & $\mathrm{C} 5$ & C6 & $\mathrm{C} 7$ & $\mathrm{C} 8$ & $\mathrm{H} 1$ & $\mathrm{H} 2$ & TI1 & TI2 & $\mathrm{TI} 3$ & TI4 & TI5 & TI6 & TI7 \\
\hline \multirow{6}{*}{$\frac{\sim}{a}$} & 1 & & & & & & & & & & & & & & & & & \\
\hline & 2 & & & & & & & & & & & & & & & & & \\
\hline & 3 & & & & & & & & & & & & & & & & & \\
\hline & 4 & & & & & & & & & & & & & & & & & \\
\hline & 5 & & & & & & & & & & & & & & & & & \\
\hline & 6 & & & & & & & & & & & & & & & & & \\
\hline \multirow{6}{*}{$\stackrel{2}{2}$} & 1 & & & & & & & & & & & & & & & & & \\
\hline & 2 & & & & & & & & & & & & & & & & & \\
\hline & 3 & & & & & & & & & & & & & & & & & \\
\hline & 4 & & & & & & & & & & & & & & & & & \\
\hline & 5 & & & & & & & & & & & & & & & & & \\
\hline & 6 & & & & & & & & & & & & & & & & & \\
\hline
\end{tabular}

Fuente: Elaboración propia

\subsubsection{Pérdidas de producto en origen}

En el capítulo 5 se planteó que las soluciones de DERTI tienen efectos desfavorables en la pérdida total de producto en algunos nodos de origen debido a la exclusión de estos nodos del diseño de la red. Para el DSRTI se incorporó en el objetivo social el criterio de pérdidas de producto con la finalidad de contrarrestar estos efectos y favorecer la participación en el mercado de los agricultores de todos los nodos de origen. Por tanto, se analiza la incidencia de las pérdidas de producto en los resultados obtenidos con el MPLEM multi-objetivo.

Las Figuras 7.10. y 7.11. muestran el porcentaje de pérdidas en cada nodo de origen para las soluciones Pareto-óptimas obtenidas con P12 y P25. En ambas Figuras se distingue la influencia de las preferencias asignadas en el comportamiento de las pérdidas de producto y en los nodos de origen que las asumen. Con P12 el nodo de origen 3 queda desvinculado del DSRTI en cuatro de las seis soluciones, principalmente, cuando el mayor peso se asigna al objetivo social o medioambiental. En la solución 4 todos los nodos de origen participan en el mercado, lo que indica que el vector de pesos empleado para esta solución es referente adecuado si se desea compensar los efectos que se ocasionan con las pérdidas de producto. Con P25 la distribución de las pérdidas cambia, dependiendo de los pesos asignados las pérdidas se concentran mayoritariamente en los nodos 3, 5 o 6 . En las soluciones 4 y 6 intervienen todos 
los nodos en el mercado y las pérdidas se distribuyen en la mitad de los nodos. Esto se logra asignando la mayor preferencia al objetivo económico o social.

Figura 7.10. Porcentaje de pérdidas de producto en los nodos de origen con P12

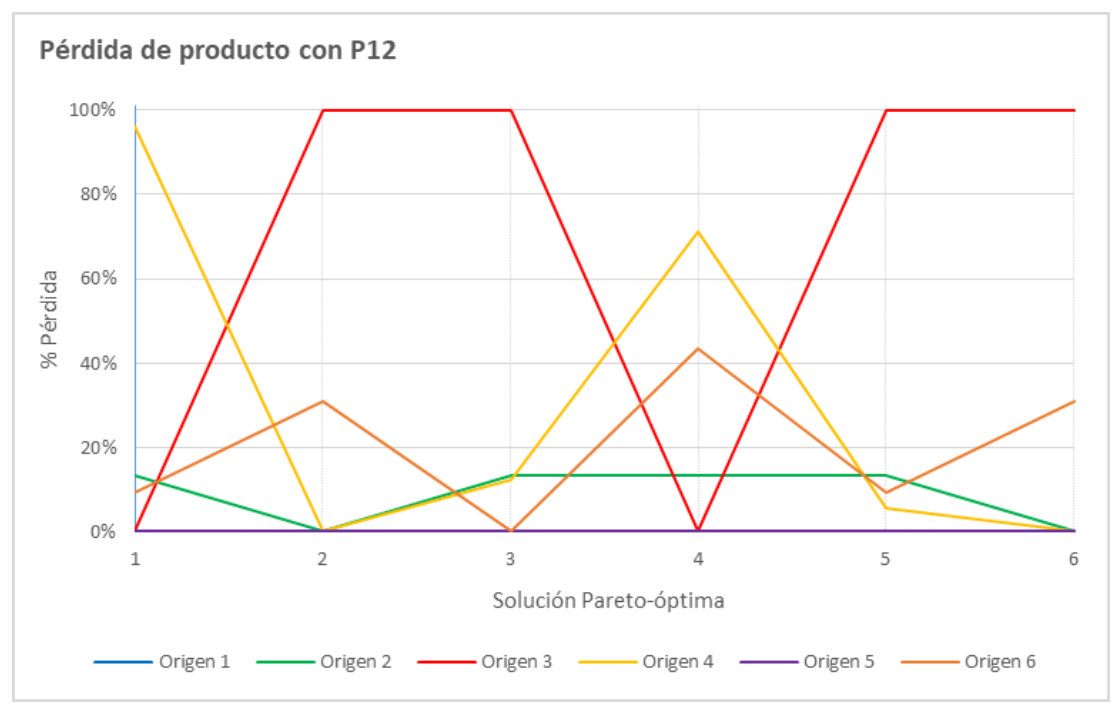

Fuente: Elaboración propia

Figura 7.11. Porcentaje de pérdidas de producto en los nodos de origen con P25

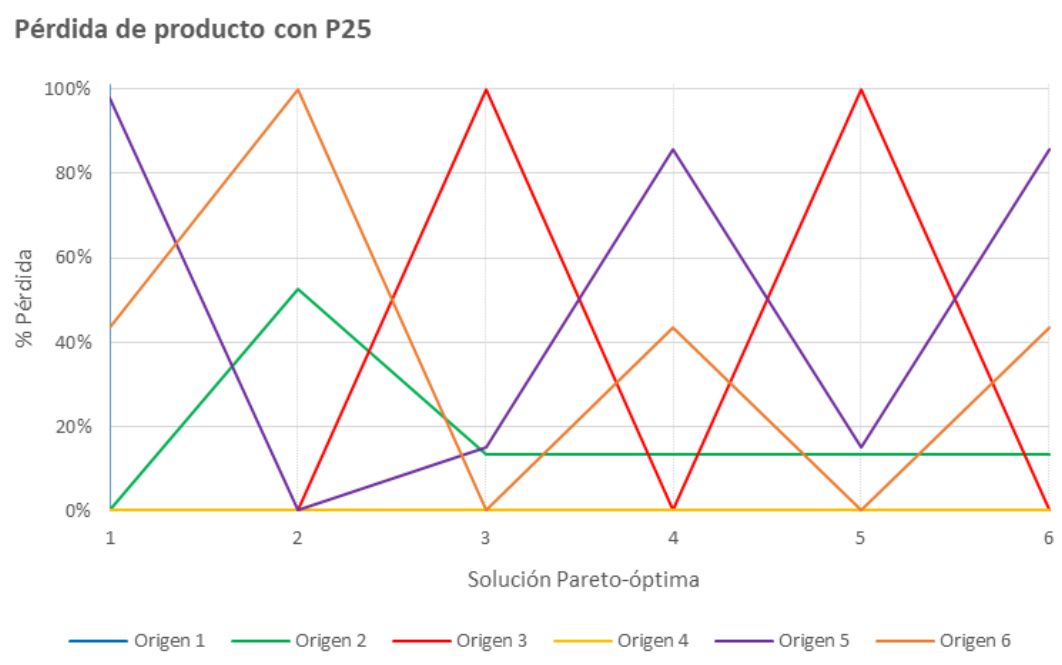

Fuente: Elaboración propia

\subsection{Discusión}

El MPLEM multi-objetivo desarrollado en este capítulo está formulado a partir de criterios que integran las tres dimensiones de sostenibilidad: económico, social y medioambiental, con los cuales se pretende, en conjunto, impulsar la actividad económica y el beneficio social a la vez que se preserva el patrimonio natural de la zona de estudio. Esta propuesta se diferencia de las existentes en la literatura no solo por incorporar las tres dimensiones de sostenibilidad en la 
formulación, sino por plantear nuevos criterios de optimización para evaluar la toma de decisiones estratégica en la priorización de proyectos de inversión con influencia en el campo del transporte. Estos criterios son: IPM, IS, PP y PB.

Aunque los proyectos de inversión tienen un impacto social favorable con los cual se asumen mejoras en los tiempos de viaje de la ciudadanía y mayor accesibilidad al equipamiento social, por sí mismos no reducen los niveles de pobreza y demás problemáticas sociales de la zona de estudio. Es necesario que se compaginen estos resultados con los presupuestos destinados a la educación y la salud, así como el desarrollo de programas agrícolas y la participación activa de la sociedad para confirmar las decisiones. El impacto ambiental desde el enfoque propuesto en esta tesis, merece mayor atención en la planificación de las decisiones estratégicas, puntualmente, en lo relacionado con la protección de la biodiversidad y el uso de suelos. La incorporación de estos aspectos asegura la continuidad de las especies y el secuestro de carbono de manera natural. Esta perspectiva no resta el compromiso de todas las partes interesadas en reducir las emisiones contaminantes en todas las formas propuestas en la literatura, por lo que es necesario desarrollar políticas y programas que dirijan las acciones tanto a la cadena de suministro como a la cadena de transporte.

Por otra parte, los tomadores de decisión pueden no comprometerse a gestionar y garantizar presupuestos que, aunque generen un impacto social, no dinamizan la economía de la región, además, se enfrentan a un gran reto para convenir la clase de transformación deseada en el territorio. Ante esto, los límites de inversión admisibles ofrecen valores óptimos más ajustados a la realidad que permiten a los tomadores de decisiones, no solo trabajar sobre metas alcanzables sino también establecer un planteamiento más acertado de las preferencias de cada objetivo. Los tomadores de decisión pueden definir las preferencias ya sea por el riesgo de los proyectos, desarrollo económico de la región, estabilidad de ecosistemas, atracción y movilidad espacial, entre otros. No obstante, la determinación de la limitación de presupuesto demuestra que no es aconsejable considerar un mismo vector de pesos (preferencias) para cualquier disponibilidad de inversión. El conflicto entre los objetivos se puede atenuar o agudizar según la disposición de los recursos económicos.

El MPLEM multi-objetivo se puede extender a futuro en distintas direcciones. Por ejemplo, la consideración de otros actores como empresas de transformación del producto agrícola y múltiples productos con enfoque de seguridad alimentaria. También puede ser utilizado como herramienta para la selección de proyectos de inversión no homogéneos, incorporando en la formulación los nuevos conjuntos de instalaciones y las matrices de conexión. En un contexto de múltiples periodos y de incertidumbre es posible analizar escenarios de demanda y de presupuesto de inversión, al igual que incluir nuevos criterios sociales relacionados con la migración de la población y medioambientales como la recuperación de la biodiversidad. Por último, la técnica de resolución se puede combinar con técnicas multi-atributo como AHP o TOPSIS para definir la importancia de cada criterio de la formulación y establecer las preferencias de los objetivos. Se reconoce que para la validación del modelo se pueden emplear otras técnicas como la suma de ponderación adaptativa, restricción-e aumentada o algoritmos evolutivos para definir las soluciones Pareto-optimas de manera más eficiente. 


\subsection{Conclusiones}

El MPLEM multi-objetivo que se desarrolló y utilizó en este capítulo satisface de manera simultánea los tres enfoques de sostenibilidad: económico, social y medioambiental, del problema combinado LI-DR intermodal. El modelo minimiza los costes y el impacto medioambiental y maximiza el beneficio social. Para la dimensión económica se mantuvieron los criterios definidos en la función objetivo presentada en el capítulo 5. Para la dimensiones social y medioambiental se definieron criterios acordes con las características de la zona de estudio, considerando también el marco de metas descrito en la Agenda 2030 y los hallazgos del análisis de la literatura. La pobreza multidimensional, la inclusión social y la pérdida de producto son el conjunto de criterios que integran la función objetivo social. La preservación de la biodiversidad y el suelo agrícola productivo son los criterios elegidos para la función objetivo medioambiental.

Este modelo aporta al estado de la cuestión, ya que los enfoques de los objetivos social y medioambiental han sido escasamente explorados, pues los estudios que incorporan las tres dimensiones de sostenibilidad son reducidos. Además, los criterios propuestos están ajustados a la realidad estudiada, y se ha diseñado un modo de representarlos que podría incorporar otros criterios o modificar el peso de los existentes, principalmente, para los criterios PP, PB y SAP. Si bien en la literatura existe una extensa variedad de métodos para la resolución de una programación multi-objetivo e identificar el conjunto de soluciones Pareto-óptimas, la practicidad del MSP cuando hay más de dos funciones facilitó la resolución del modelo y el análisis de las preferencias de los tomadores de decisiones frente a distintos vectores de pesos y presupuestos de inversión.

El análisis permitió determinar el mínimo compromiso financiero requerido por los tomadores de decisiones para garantizar un DSRTI. También demostró que el conjunto de soluciones Pareto-optimas y el conflicto entre los objetivos varia con el presupuesto disponible. Con las fronteras de Pareto se deduce que pretender los mínimos costes de uso de la red provocan un débil beneficio social cuando el presupuesto es bajo; por el contrario, con altos presupuesto el conflicto entre estos objetivos se reduce. El objetivo medioambiental muestra compensaciones desfavorables con el objetivo económico, que se incrementan conforme se dispone más inversión; igualmente, pero en mayor medida, se replican estos efectos con el objetivo social. Para obtener el conjunto de soluciones Pareto-óptimas se validaron distintos vectores de pesos en la optimización de la función global. Los resultados revelan la correspondencia de la asignación de las preferencias con el presupuesto disponible, ya que con más presupuesto se amplía el número de proyectos localizables y con ello las posibilidades de moderar el conflicto entre los objetivos. 


$$
\text { CAPÍtULO } 8
$$




\section{Capítulo 8}

\section{Conclusiones generales}

Presentación: en este capítulo se presentan las conclusiones generales de la tesis. Las conclusiones ahondan en las principales contribuciones y limitaciones al trabajo realizado, las oportunidades identificadas para el desarrollo de futuras líneas de investigación, se proponen algunas consideraciones para las administraciones públicas y por último se presenta la trayectoria seguida por la autora durante su etapa formativa doctoral. 


\subsection{Introducción}

En este capítulo se presentan las conclusiones finales de la tesis. Estas se organizan de la siguiente manera. En la sección 8.2. una síntesis de lo realizado en los distintos capítulos subrayando las principales contribuciones y limitaciones al trabajo realizado. En la sección 8.3. se describen las oportunidades identificadas para el desarrollo de futuras líneas de investigación. En la sección 8.4. se proponen algunas consideraciones para los actores y grupos de interés. En la sección 8.5. se presenta la trayectoria seguida por la autora durante su etapa formativa doctoral.

\subsection{Contribuciones y limitaciones}

La tesis se organizó en seis capítulos principales, que fueron precedidos por el capítulo de introducción general y que finaliza con el presente capítulo de conclusiones generales. Por lo tanto, para una mejor disposición y seguimiento de las contribuciones y limitaciones al trabajo realizado, estas, se diferencian para cada capítulo de contenido teórico o analítico (de este modo no se contempla el capítulo 2). A su vez se comentan las limitaciones encontradas en estos.

En el Capítulo 3 se aporta el enfoque metodológico de programación matemática utilizado para el análisis de las decisiones. Para ello se siguieron deducidos de la literatura para efectuar la transformación del problema real a un problema tratable con las técnicas de programación matemática. El proceso de transformación permitió acotar adecuadamente el marco de decisiones para proponer soluciones al problema real de acuerdo con la disponibilidad de datos para analizarlo. La identificación de este marco facilitó la detección del problema de toma de decisiones, es decir, del problema combinado de localización de instalaciones-diseño de red (LI-DR) intermodal. Con la detección del tipo de problema de decisión se destacó la importancia de incorporar las redes de transporte subyacentes para refinar la toma de decisiones, por ende, la necesidad de definir una estructura matemática sólida y flexible en la que confluyeran todos los elementos del problema. En este caso la estructura de nodos y arcos de los grafos permitió la construcción de una red de entrada que se utilizó como base para proponer las relaciones matemáticas de los modelos de programación que se presentaron en los capítulos 5, 6 y 7.

Aunque el proceso de transformación se llevó a cabo de manera satisfactoria, la disponibilidad limitada en la variedad de datos o la posibilidad de obtenerlos atenuó el alcance del problema de investigación tanto por los criterios de optimización definidos para evaluar y comparar los proyectos de inversión, como en la planificación táctica y operativa del problema. Esta limitación no altera el tipo de problema de decisiones identificado, ya que los requerimientos en la toma de decisiones se focalizan a nivel estratégico con la selección de los proyectos de inversión. La limitación de datos relacionados, por ejemplo, con el valor económico de las pérdidas de producto, las características del parque automotor, los tiempos de servicio por modo de transporte o los flujos de pasajeros podrían tener efecto en las decisiones de asignación. No obstante, por las características combinadas del problema LI-DR la introducción de estos datos también podrían conllevar a cambios en las decisiones estratégicas. De manera similar para las dimensiones de sostenibilidad social y medioambiental, la situación real de la ZODES Magdalena Medio ofrece un conjunto de 
posibilidades para establecer enfoques que permitan valorar con más profundidad la planificación del territorio.

Por su parte, los procedimientos definidos para resolver los modelos de programación permitieron explorar dos alternativas de codificación distintas, proporcionando cierta flexibilidad para ajustar el código informático conforme a las condiciones establecidas para validar los modelos. La aplicación de Gurobi Optimizer como solucionador demostró la idoneidad de los tiempos de ejecución para su utilización en la resolución del número de instancias generadas para cada modelo de programación, así como de las soluciones obtenidas. Aunque este aspecto no fue objeto de análisis para la tesis, la afirmación anterior se comprobó con las distintas pruebas llevadas a cabo al emplear CBC MILP Solver con el esquema implementado en Python. No obstante, se desconocen los efectos en la eficiencia computacional y de las soluciones de diseño de la red.

En el Capítulo 4 se presentaron los resultados del análisis de la literatura desde dos perspectivas. En la primera se identificaron las aportaciones alrededor de los sistemas de transporte intermodal para identificar factores clave en la composición y funcionamiento del sistema, así como la caracterización en la planificación y niveles de decisión. Para ello se aplicaron los pasos propuestos por Denyer \& Tranfield (2009) para una revisión sistemática de literatura (RSL). La segunda perspectiva se focalizó en los problemas combinados LI-DR basados en el uso de técnicas de programación matemática. Para ello se identificó una oportunidad metodológica en el proceso de revisión que permitió aportar una novedosa propuesta en la literatura, al vincular tres nuevos pasos como etapa previa a los propuestos por Denyer \& Tranfield (2009). Esta propuesta novedosa se denominó revisión sistemática de la literatura en dos etapas vinculadas (RSL-2EV) y se utilizó para aportar los resultados en la segunda perspectiva. Para ambos análisis se proponen clasificaciones novedosas que facilitan la interpretación de la literatura y actualización futura del estado de la misma. Además, las lagunas de investigación identificadas en ambas revisiones, tales como la capacidad física y financiera, el enfoque de sostenibilidad o los múltiples presupuestos de inversión, permitieron perfilar los modelos de programación que se propusieron en los capítulos 5, 6 y 7 .

Con las dos perspectivas de revisión de la literatura se subsanaron posibles imprecisiones en la selección de los estudios y en los hallazgos que se aportaron, pues, más que concentrar un extenso número de estudios en un único análisis se procuró la segmentación en la exploración en las dos perspectivas debido a que, por una parte, se hizo necesario identificar los componentes de los sistemas intermodales y, por otra parte, analizar un problema de decisiones en particular. Con la RSL se cubrió satisfactoriamente el primer segmento. Los resultados no solo permitieron la identificación de los factores clave de los sistemas intermodales para enmarcar las formulaciones de los modelos propuestos. Igualmente, condujeron a definir líneas de investigación futuras en el campo de transporte intermodal.

La RSL-2EV cubrió favorablemente el segundo objetivo de la revisión de la literatura. Los problemas combinados LI-DR se rastrearon a partir de dos estrategias de búsqueda aplicadas de manera exitosa, demostrándose el potencial de estos problemas para la aplicación y desarrollo de técnicas de programación matemática. Sin embargo, el uso de estas técnicas como criterio de inclusión delimitó la exploración de otros estudios con resultados que podrían enriquecer la identificación de las características del problema combinado LI-DR y de posibles temas emergentes. Esto no indica que los resultados obtenidos de la RSL-2EV sean ambiguos, 
pues la clasificación propuesta se centra en los componentes del problema de investigación y la técnica de programación matemática. Las posibles contribuciones de los estudios que emplearon otras técnicas, como por ejemplo la simulación o técnicas multi-atributo, podrían dar cuenta de otros criterios de optimización relacionados directamente con la toma de decisiones de nivel estratégico y de análisis basados en los enfoques de sostenibilidad y toma de decisiones con incertidumbre en los datos.

En el Capítulo 5 se propuso un modelo de programación lineal entero-mixto (MPLEM) mono-periodo para diseñar la red intermodal optimizando sus costes de uso. El modelo satisface el problema combinado LI-DR intermodal desde un enfoque económico, a la vez que es extensible a otros contextos o regiones con características similares. Para formularlo se incorporó la planificación física y financiera del sistema y la importancia de los costes como factores clave en el funcionamiento de este, acorde con las oportunidades de investigación identificadas en el capítulo 4. La solución de diseño económico de la red de transporte intermodal (DERTI) se analizó parametrizando los valores de la demanda, las capacidades y el presupuesto de inversión. Los resultados reflejan un comportamiento aceptable en las soluciones de DERTI. Dado que la disponibilidad de financiación es una limitante al diseño, se identificó un rango de disponibilidades factibles cuyos efectos proporcionan información vital para guiar la toma de decisiones de las administraciones públicas. El análisis identificó los desequilibrios que se ocasionan en la región por las pérdidas de producto lo que llevó a plantear una variante al modelo para compensar estos efectos. Los resultados de esta variante demostraron la significativa sensibilidad de los diseños ante los cambios en las pérdidas de producto admitidas en cada nodo de origen, cuando se consideró una amplia capacidad física en los proyectos intermodales.

El MPLEM mono-periodo permitió obtener soluciones estratégicas de DERTI que, además de satisfacer los requerimientos en el cumplimiento de la demanda, satisface las necesidades de la nueva infraestructura y los objetivos de los decisores de la cadena de suministro agrícola, la cadena de transporte y de los actores gubernamentales de la ZODES Magdalena Medio. Como se mencionó anteriormente, la limitación en la variedad de datos para definir los criterios de optimización atenuó el alcance del problema de investigación y con ello el sentido de la función objetivo propuesta. Una formulación con sentido en la maximización de los beneficios podría acarrear mayor participación de los agricultores, especialmente, por las pérdidas de producto que asumen, así como evaluar la factibilidad de los proyectos en función del coste de oportunidad entendida desde la limitada disponibilidad de los recursos económicos por parte de las administraciones públicas.

La sensibilidad a nivel estratégico de las soluciones de DERTI demostró la solidez en la estructura de la red de entrada construida, aunque los análisis no permiten obtener resultados concluyentes respecto a la flexibilidad de esta estructura. El conjunto de nodos y arcos de las redes de transporte subyacentes proporcionados, para el modelo propuesto en este capítulo y para los modelos propuestos en los capítulos 6 y 7, no se modificó. Una modificación a la estructura de la red, bien sea agregando un nuevo arco o suprimiéndolo, podría presentar soluciones de DERTI con distintas características en la configuración intermodal y, por consiguiente, distintos costes de uso de la red intermodal. Mientras, la sensibilidad a nivel táctico de las soluciones de DERTI, específicamente en las infraestructuras de transporte de primera milla, corroboran la necesidad de caracterizar las capacidades de los vehículos modales para profundizar en el análisis de uso de la red. La asignación de los flujos basada únicamente 
en los costes no permite capturar los desequilibrios que se pueden ocasionar en esta decisión con la utilización de un tipo de vehículo especifico.

En el Capítulo 6 se propuso la extensión multi-periodo del MPLEM presentado en el capítulo 5 para analizar la sensibilidad de las soluciones de DERTI en un horizonte de planificación a largo plazo y se incorporaron dos condiciones a la formulación con la finalidad de validar los resultados. Las soluciones estratégicas de DERTI en múltiples periodos de decisión permite identificar el orden de preferencia en la selección y programación de los proyectos de inversión, a medida que cambian los costes de transporte por modo y la disponibilidad de los recursos financieros en cada periodo. Los análisis reflejan qué, para la ZODES, los costes de transporte fluvial tienen más influencia en la sensibilidad de la solución provocando cambios sustanciales en el listado de proyectos de inversión priorizados. La validación de las condiciones de interdependencia entre los proyectos de inversión y de las múltiples fuentes de financiación guardan estrecha relación con el rol de las administraciones públicas en la ZODES. En este sentido ambas condiciones son funcionales y coherentes con el problema combinado LI-DR, específicamente para la segunda condición se demostró que más participación del actor público no conlleva a mejores costes óptimos y que la participación de inversores privados es viable siempre que controle su intervención.

El MPLEM multi-periodo es de gran utilidad en la toma de decisiones para priorizar los proyectos de inversión financiables, sin embargo, se podría valorar la reestructuración del supuesto que permite el uso del proyecto desde el mismo periodo que se priorizó. La aplicación de este supuesto no invalida los resultados analizados en el capítulo, puesto que, para los proyectos de primera milla, se evaluó una sola opción de inversión (desplegada en tres alternativas secuenciadas) en casi todos los nodos de origen. La reestructuración del tal supuesto podría dirigirse en considerar los periodos de inactividad en la construcción de los proyectos según el modo de transporte. Los tiempos de inactividad pueden tener mayor influencia en las decisiones tácticas que en las estratégicas, ya que al imponer el periodo de inactividad se pueden usar las vías de transporte actuales mientras se construyen los proyectos de primera milla. La distinción de los tiempos de inactividad por modo de transporte parece atractiva porque se consideran tres redes modales subyacentes diferentes $\mathrm{y}$, por tanto, el orden de preferencia de los proyectos priorizados posiblemente se vería alterado.

Además de lo anterior, con el MPLEM multi-periodo con interdependencias entre los proyectos de inversión parece interesante efectuar una validación con otra estructura de red para comprobar si los resultados obtenidos tienden a la unificación de la configuración intermodal de la red. Para el MPLEM multi-periodo con interdependencias y con los múltiples presupuestos de inversión, si bien se demuestran las ventajas económicas de financiar los proyectos de inversión con fuentes privadas, el coste social considerado podría tener un alcance limitado frente a la rentabilidad esperada por parte de este inversor. Por tanto, la métrica del coste social podría basarse en cálculos estimados de la tasa de retorno de la inversión para cada proyecto.

En el Capítulo 7 se propuso un MPLEM multi-objetivo para abordar el problema combinado LI-DR intermodal optimizando de manera simultánea las tres dimensiones de sostenibilidad: económica, social y medioambiental. Los condiciones sociales y medioambientales identificados en el problema de investigación se definieron como criterios de optimización que se agruparon e introdujeron en dos nuevas funciones objetivo al MPLEM 
propuesto en el capítulo 5. Los tres enfoques se direccionaron de tal modo que pretenden, en conjunto, impulsar la actividad económica y el beneficio social a la vez que se preserva el patrimonio natural. Este modelo supone una contribución a la literatura ya que los enfoques social y medioambiental han sido escasamente explorados en la literatura del campo del transporte. Además, se distinguen significativamente por la propuesta novedosa en los criterios de optimización definidos desde una óptica externa, pues para estos no se estableció una vinculación directa con las decisiones tácticas de asignación. El problema de investigación tiene un gran número de componentes que permitirían precisar otros criterios de optimización en cada etapa de la cadena de suministro para integrarlos a estas funciones, permitiendo que en cada proyecto también se evalúe la convergencia de la óptica externa asumida y una óptica interna de las organizaciones.

El análisis de las soluciones para el diseño social de la red intermodal (DSORTI) y el diseño medioambiental de la red intermodal (DMRTI) se analizó variando los parámetros de demanda y presupuesto de inversión. Los DSORTI y DMRTI demuestran la incompatibilidad de los objetivos y la necesidad de definir límites de inversión admisibles para realizar los análisis de las soluciones Pareto óptimas. Aunque en la practica la determinación de este rango de inversión factible puede ser adecuado, ofrece a la actividad económica cierta prioridad sobre los otros dos enfoques. Una determinación más afín a la realidad podría ser definiendo el limite desde el valor objetivo que persigan los decisores. Es posible que con esta forma se planteen nuevos grados de conflicto entre los objetivos, especialmente con el objetivo medioambiental, para las disponibilidades de financiación analizadas. Esto implicaría para cada presupuesto de inversión un conjunto de soluciones Pareto-óptimas distintas a las presentadas en este capítulo.

Para resolver el MPLEM multi-objetivo se llevó a cabo un análisis para definir estrategias en la asignación de preferencias (vectores de peso) a cada objetivo para aplicar el método de suma ponderada (MSP). Con estas se demostró que el conjunto de soluciones Pareto-optimas resueltas para el diseño sostenible de la red intermodal (DSRTI) y el conflicto entre los objetivos varía según la disponibilidad de financiación, y que un mismo grupo de preferencias asignadas a los objetivos tienen efectos que difieren según la disponibilidad financiera para tomar las decisiones. Aun cuando el MSP permite la optimización simultánea de las tres funciones objetivo, previa normalización de los datos, y con los análisis presentados se comprueba la utilidad del modelo. Si bien se demuestra tal utilidad, el MSP no permite hallar el conjunto de las soluciones Pareto-óptimas en tiempos razonables, encontrando solo un número determinado de éstas. Ello más que una limitación se puede entender como una debilidad subsanable en el esquema de resolución, considerando la construcción de algoritmo que evalúe los vectores de pesos y clasifique las soluciones del conjunto Pareto-óptimo.

\subsection{Líneas futuras de investigación}

En cada etapa de la tesis emergieron cuestiones de investigación que requirieron ser discutidas con el propósito de precisar el alcance de las actividades planificadas y los resultados esperados. Con cada discusión se fue perfilando la estructura de la tesis presentada en este documento. Las cuestiones, más allá de ser aceptadas o rechazadas, permitieron identificar un conjunto de oportunidades para enriquecer el trabajo presentado y otro conjunto para la futura exploración y desarrollo de líneas de investigación. En esta sección se exponen las líneas de investigación con potencial de exploración para dar continuidad al trabajo de investigación y 
enmarcar la carrera investigadora de la autora de la tesis. Algunas de estas líneas se derivan de las limitaciones discutidas en la sección anterior.

La estructura de la red de entrada constituye una ruta de acción con la cual es posible validar no solo la flexibilidad de la misma desde la perspectiva de sensibilidad del diseño de red, ya sea reduciendo o ampliando el número de nodos y arcos proporcionados, sino también bajo escenarios hipotéticos de ocurrencia de situaciones comerciales y políticas que condicionen la toma de decisiones, especialmente de tipo estratégico. De igual manera, a partir de estructuras de redes de distintas dimensiones sería interesante comprobar la utilidad de los modelos propuestos y realizar comprobaciones y comparaciones computacionales entre estas. Desde la perspectiva de las características de la red, en el futuro se podría introducir la categorización de los nodos y los arcos para combinar en la red las conexiones locales, regionales y nacional con el propósito de analizar el impacto del diseño de la red en función de la planificación del territorio y las redes de transporte integradas.

En relación con el problema de investigación y su formulación, la investigación futura se propone en varios sentidos: los objetivos de optimización y los problemas de decisión. En cuanto al primero, se propone extender las formulaciones atendiendo a los factores clave identificados para mejorar la eficiencia del sistema y la calidad del servicio. En este grupo se contemplan tiempos de transporte, tiempos de operación en las terminales intermodales, tiempos de inactividad de las infraestructuras durante la construcción, al igual que costes por pérdidas de producto, costes sociales, estacionalidad del producto, capacidades en los arcos, entre otros. También la diversificación de criterios de los enfoques social y medioambiental utilizados. La incertidumbre de los datos asociados a los diferentes escenarios de ocurrencia para evaluar la viabilidad y optimización de soluciones, tanto como los efectos en la volatilidad del presupuesto de inversión también pueden ser líneas prometedoras para explorar y desarrollar.

En cuanto al segundo ámbito, para el modelado de las decisiones de localización y asignación del problema combinado LI-DR intermodal sería conveniente poner mayor énfasis en las implicaciones de la pérdida de producto, no solo en los nodos de origen sino en las distintas etapas de la cadena debido a la característica perecedera del producto. Además, los modelos propuestos son adaptables para estudiar otros problemas de decisión que, desde el contexto de la planificación urbana y territorial, permiten analizar las decisiones en problemas de localización de múltiples tipos de instalaciones, localización jerárquica de instalaciones, tanto como en la planificación estratégica de los sistemas de transporte urbanos.

Para los métodos y procedimientos de resolución se propone consolidar los esquemas de resolución empleados en esta tesis utilizando otros solucionadores como Local Solver o CBC MILP Solver que permitan realizar análisis comparativos respecto a la eficiencia computacional. De igual manera, extender los procedimientos construyendo heurísticas que respondan rápidamente a la optimización personalizada y a escala de los datos del modelo, así como la aplicación de programación estocástica en dos etapas para la validación y optimización basada en la incertidumbre de la demanda y la disponibilidad de financiación. Para la resolución de modelos multi-objetivo sería conveniente utilizar otras técnicas como la suma ponderada adaptativa, restricción-e aumentada o algoritmos evolutivos con la finalidad de definir las soluciones Pareto-optimas de una manera más eficiente. 
Respecto a la sostenibilidad y criterios de optimización sería importante realizar una revisión de la literatura respecto a los criterios de sostenibilidad estudiados en el diseño de redes de transporte, en preferencia los criterios sociales y medioambientales (distintos a las emisiones), para determinar el grado de implicación de estos enfoques en la toma de decisiones, así como la activa participación de la sociedad y los grupos de interés. De acuerdo con la óptica externa que se propuso para esta tesis, esta puede complementarse con decisiones de restauración de la biodiversidad como con criterios de seguridad alimentaria basados en las pérdidas de producto. Desde la óptica interna de las organizaciones la colaboración y cooperación entre los actores y la sincronización de las actividades y procesos entre las etapas de la cadena de suministro también son líneas futuras prometedoras. Esta línea también alberga la utilización de técnicas multi-atributo como AHP, TOPSIS, DEMATEL y PROMETHEE como método complementario para validar las métricas, determinar la importancia relativa entre los criterios de un mismo enfoque, establecer los pesos en las preferencias de los decisores o clasificar las soluciones del conjunto Pareto-óptimo.

\subsection{Implicaciones gerenciales: Administraciones públicas}

La respuesta a la pregunta de investigación que direccionó el objetivo general de esta tesis proporciona información vital para las administraciones públicas, pues, da la contestación a ciertos interrogantes que estos se harían antes de tomar la decisión de invertir en grandes proyectos de infraestructura. Entre estas, la consideración en la evaluación conjunta de dos tipos de proyectos de inversión para gestionar la cartera de proyectos, los aspectos críticos para hacer más eficiente la asignación del presupuesto de inversión o la participación de actores privados como financiadores de los proyectos. Todas estas con una visión integral y sostenible en la transformación de la región a partir del fortalecimiento de las cadenas de suministro, el beneficio a la sociedad y el medioambiente. Si bien se reconoce que las inquietudes están interrelacionadas y tienen efectos recíprocos, los análisis presentados tienen diversas implicaciones gerenciales para las administraciones públicas. Los resultados, aunque son tendenciosos a la situación de la ZODES Magdalena Medio, ofrecen elementos que los hacen extensibles a otras regiones.

Una de las principales implicaciones gerenciales destaca la identificación adecuada del problema de decisiones como garante para evaluar las estrategias de inversión. Por una parte, los gestores deben reconocer el potencial de los sistemas intermodales para mejorar el desempeño logístico de la ZODES, las características geográficas facilitarían la implementación de estos sistemas con una significativa reducción de los costes que permitiría incrementar la competitividad de la cadena de suministro agrícola e impulsar el desarrollo de la región. No obstante, las bondades que se podrían obtener están sujetas a acciones complementarias encaminadas a fortalecer la cadena agroalimentaria a través de programas que aseguren la oferta de productos. Por otro parte, el reconocimiento de las decisiones críticas para el problema conduce a establecer un marco de análisis con una utilidad práctica. En este caso la evaluación de los dos tipos de proyectos de inversión, la etapa de la cadena de suministro que se analiza y los intereses de los distintos actores y grupos permiten confluir aspectos que en ocasiones son abordas de manera independiente. Desde esta óptica, la colaboración y participación institucional de las administraciones públicas es fundamental y 
requiere de establecer compromisos y acercamientos tanto con los actores de la cadena de suministro y de la cadena de transporte, como con la sociedad y los inversores privados.

Por lo anterior, los modelos de optimización propuestos tienen un gran potencial como herramienta de soporte para la toma de decisiones en las administraciones públicas. El análisis de soluciones realizado garantiza la utilidad de los mismos. Los modelos ofrecen posibilidades de mejorar la eficiencia en la asignación de los recursos económicos a la luz de las capacidades del sistema, la demanda y la disponibilidad de financiación. Reconociendo la complejidad inherente a la realidad, con estos modelos se puede continuar la construcción gradual de la formulación. Sin embargo, los gestores públicos enfrentan un gran desafío para asegurar la funcionalidad y validez de las soluciones obtenidos. Este desafío apunta a una gestión adicional que procure la obtención y depuración de los datos necesarios para validar los nuevos modelos. En especial los referidos a las actividades realizadas en las zonas de cultivo, las estrategias de comercialización y la participación de los intermediarios, abordados desde los tres enfoques de sostenibilidad.

\subsection{Trayectoria investigadora de la doctoranda}

En esta sección se realiza un breve recorrido de mi trayectoria en la etapa de formación doctoral que contribuyeron en el desarrollo de mi carrera como investigadora, desde la incorporación al programa de doctorado en Administración y Dirección de Empresas (ADE) de la Universitat Politècnica de València (UPV).

Este recorrido inició en septiembre de 2016, con el apoyo de la Fundación CEIBA y la Gobernación de Bolívar (Colombia) al concederme una de las Becas Condonable del programa de formación de talento humano "Bolívar Gana con Ciencia". La recepción en el Departamento de Organización de Empresas de la UPV, por parte de mi Director de tesis, y la interacción con el personal docente-investigador y con otros doctorandos, facilitó mi transición socio-cultural y la adaptación al ambiente laboral. Además, es conveniente resaltar el constante respaldo recibido por mi co-director de tesis y del Departamento de Organización de Empresas, Marketing y Sociología de la Universidad de Jaén (UJA).

Las actividades desarrolladas se centraron, inicialmente, en cumplir las horas de formación transversal exigidas por el programa de doctorado en ADE, en conjunto con la elaboración del plan de investigación y la revisión de la literatura. Estas actividades permitieron el fortalecimiento de mis habilidades y capacidades en la comprensión e identificación de problemas en el campo del transporte y su delimitación conforme con los distintos grados de complejidad en los procesos de toma de decisiones, así como la cualificación en el uso de las bases de datos científicas con sentido pragmático y utilitario y el trabajo multidisciplinar. A partir de los resultados parciales obtenidos del cumplimiento de las actividades del plan de investigación, se alternó la elaboración de propuestas de artículos científicos con la difusión de los avances en congresos, jornadas doctorales y presentaciones en el interior de la comunidad académica de la UPV y la UJA. Estas actividades permitieron el continuo fortalecimiento de la comunicación oral y escrita en contextos académicos, y el desarrollo de redes de conocimiento y colaboración con investigadores nacionales e internacionales. A continuación, se sintetizan los resultados obtenidos como producto de las experiencias descritas. 

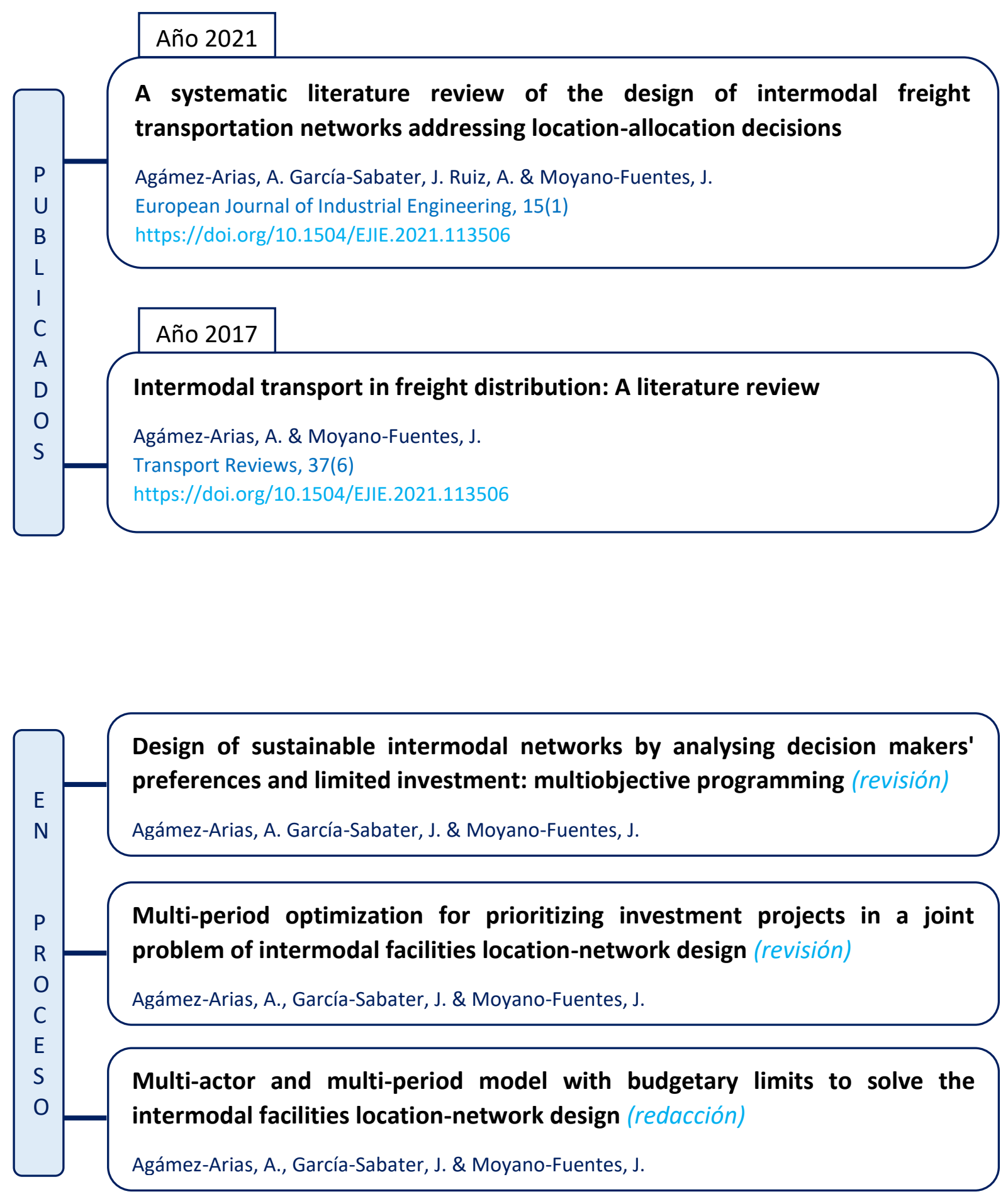


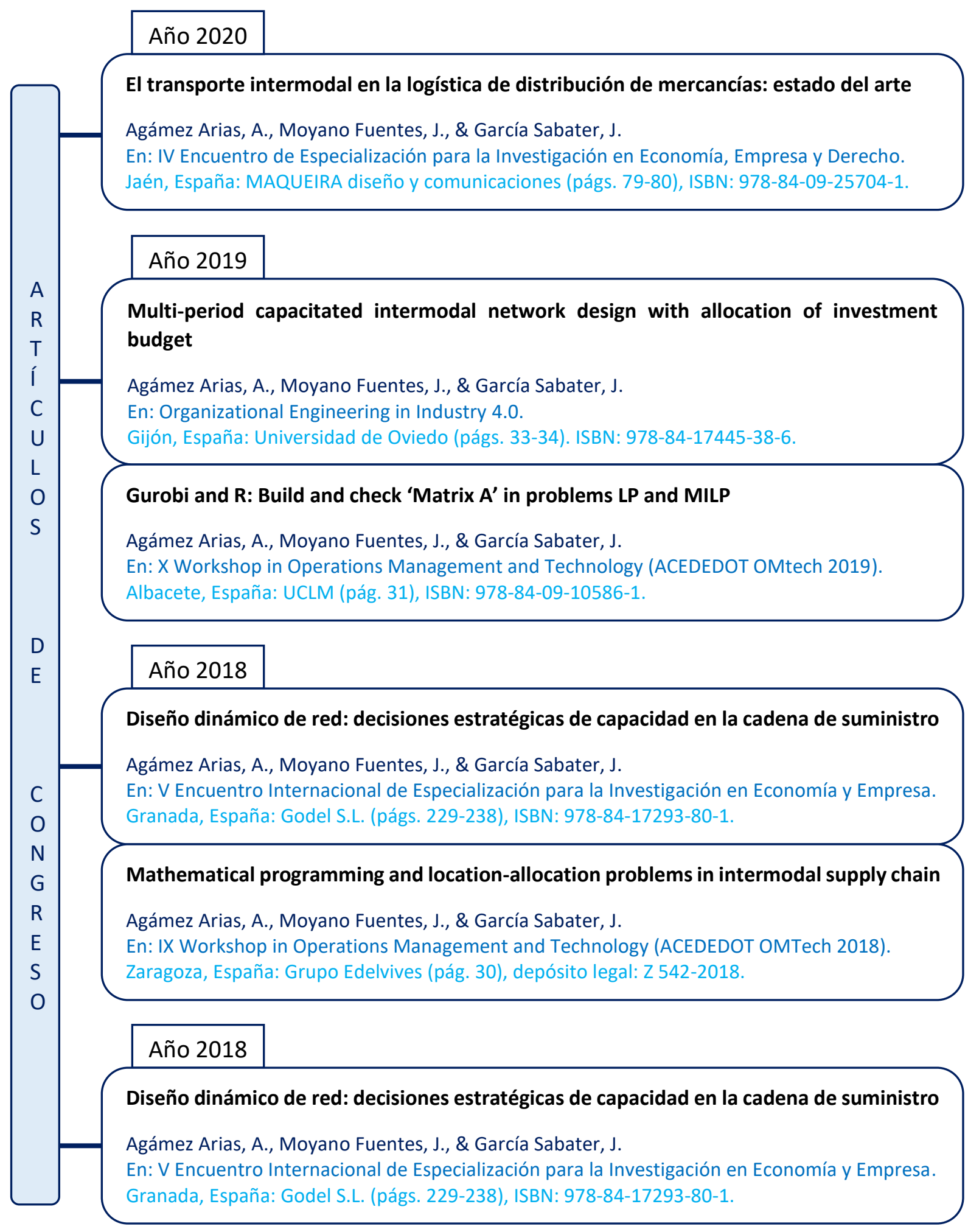


- Comunicaciones a congresos

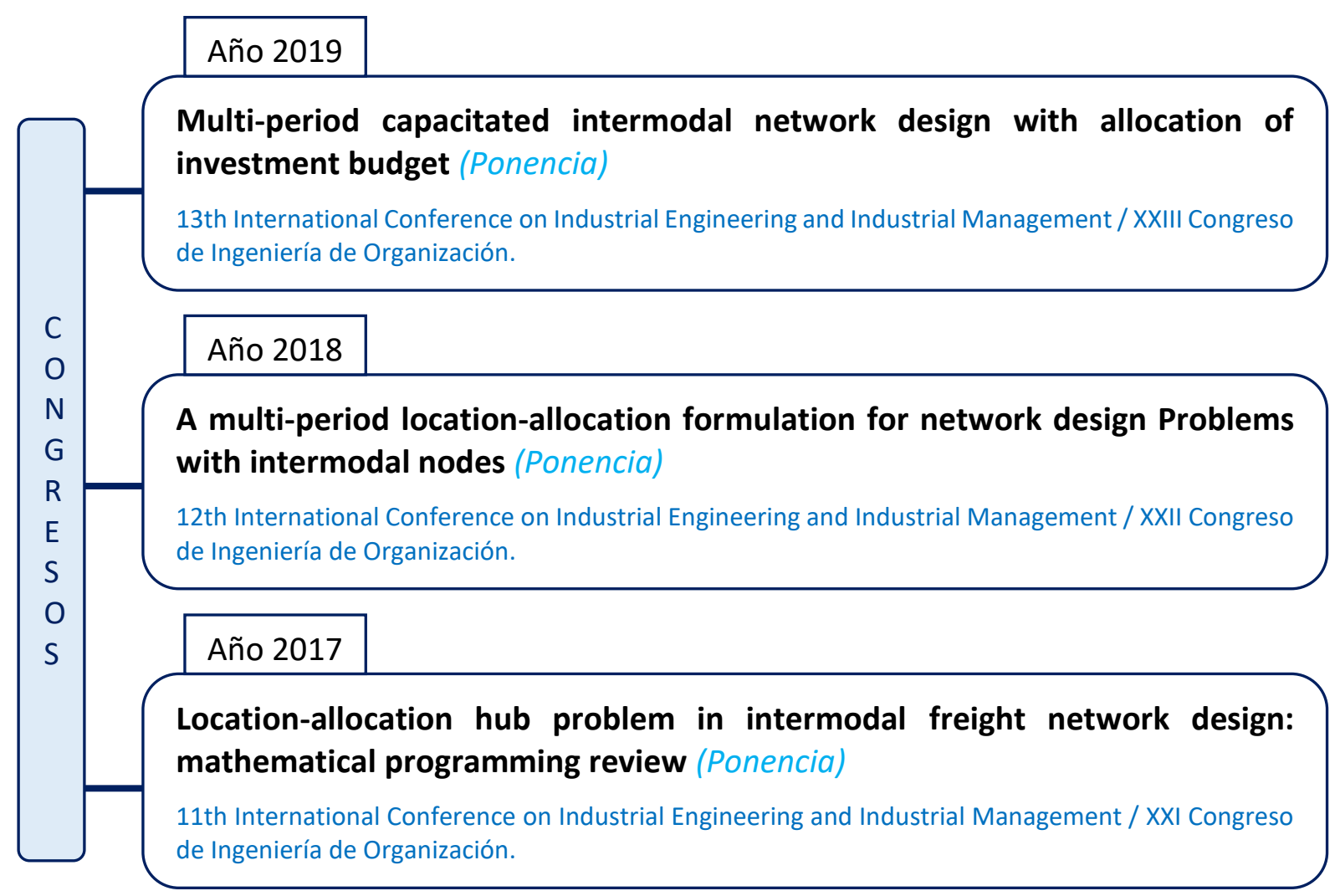




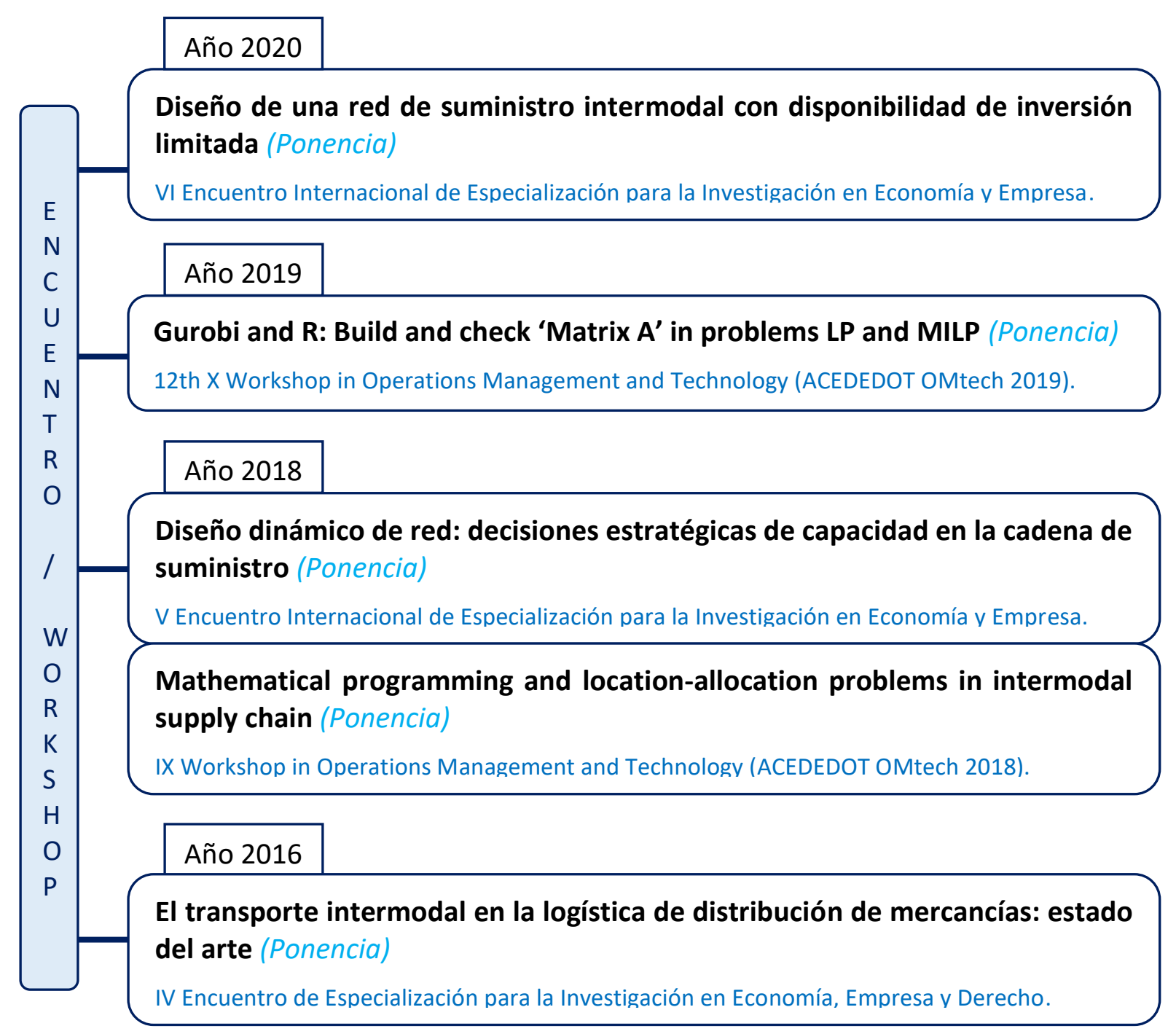




\section{Referencias}

Agámez-Arias, A., García-Sabater, J., Ruiz, A. \& Moyano-Fuentes, J. (2021). A systematic literature review of the design of intermodal freight transportation networks addressing location-allocation decisions. European Journal of Industrial Engineering, 15(1), 1-34. https://doi.org/10.1504/EJIE.2020.10031931

Agámez-Arias, A. \& Moyano-Fuentes, J. (2017). Intermodal transport in freight distribution: A literature review. Transport Reviews, 37(6), 782-807. https://doi.org/10.1080/01441647.2017.1297868

Agencia Nacional de Infraestructuras. (15 de junio de 2021). Mapas de carreteras, puertos y ferrocarriles. https://www.ani.gov.co/

Ahumada, O. \& Villalobos, J. (2009). Application of planning models in the agri-food supply chain: A review. European Journal of Operational Research, 196(1), 1-20. https://doi.org/10.1016/j.ejor.2008.02.014

Akgün, İ. \& Erdal, H. (2019). Solving an ammunition distribution network design problem using multi-objective mathematical modeling, combined AHP-TOPSIS, and GIS. Computers \& Industrial Engineering, 129, 512528. https://doi.org/10.1016/j.cie.2019.02.004

Alarcón, S., Marcucci, D. \& Quiroga, M. (2018). Territorialidad campesina y agroindustria en el río Cimitarra. Bitacora Urbano Territorial, 3, 181-188. https://doi.org/10.15446/bitacora.v28n3.72206

Alenezi, A. \& Darwish, M. (2014). Integrated location model with risk pooling and transportation mode selection. International Journal of Industrial and Systems Engineering, 16(2), 200. https://doi.org/10.1504/IJISE.2014.058836

Alizadeh, M., Mahdavi, I., Mahdavi-Amiri, N. \& Shiripour, S. (2015). A capacitated location-allocation problem with stochastic demands using sub-sources: An empirical study. Applied Soft Computing, 34, 551-571. https://doi.org/10.1016/j.asoc.2015.05.020

Alkire, S. \& Santos, M. (2014). Measuring acute poverty in the developing world: Robustness and scope of the multidimensional poverty index. World Development, 59, 251-274. https://doi.org/10.1016/j.worlddev.2014.01.026

Allaoui, H., Guo, Y., Choudhary, A. \& Bloemhof, J. (2018). Sustainable agro-food supply chain design using two-stage hybrid multi-objective decision-making approach. Computers \& Operations Research, 89, 369384. https://doi.org/10.1016/j.cor.2016.10.012

Almotairi, B., Flodén, J., Stefansson, G. \& Woxenius, J. (2011). Information flows supporting hinterland transportation by rail: Applications in Sweden. Research in Transportation Economics, 33(1), 15-24. https://doi.org/10.1016/j.retrec.2011.08.003

Alstadt, B., Weisbrod, G. \& Cutler, D. (2012). Relationship of transportation access and connectivity to local economic outcomes. Transportation Research Record, 2297(1), 154-162. https://doi.org/10.3141/2297-19

Alumur, S. \& Kara, B. (2008). Network hub location problems: The state of the art. European Journal of Operational Research, 190(1), 1-21. https://doi.org/10.1016/j.ejor.2007.06.008

Alumur, S., Kara, B. \& Karasan, O. (2012). Multimodal hub location and hub network design. Omega, 40(6), 927-939. https://doi.org/10.1016/j.omega.2012.02.005

Alumur, S., Nickel, S., Saldanha-da-Gama, F. \& Seçerdin, Y. (2016). Multi-period hub network design problems with modular capacities. Annals of Operations Research, 246(1-2), 289-312. https://doi.org/10.1007/s10479015-1805-9

Álvarez-Miranda, E., García-Gonzalo, J., Pais, C. \& Weintraub, A. (2019). A multicriteria stochastic optimization framework for sustainable forest decision making under uncertainty. Forest Policy and Economics, 103, 112122. https://doi.org/10.1016/j.forpol.2018.03.006

Amaral, M., Almeida, M. \& Morabito, R. (2012). Um modelo de fluxos e localização de terminais intermodais para escoamento da soja brasileira destinada à exportação. Gestão \& Produção, 19(4), 717-732. https://doi.org/10.1590/S0104-530X2012000400005

Ambra, T., Caris, A. \& Macharis, C. (2019). Should I stay or should I go? Assessing intermodal and synchromodal resilience from a decentralized perspective. Sustainability, 11(6), 1765. https://doi.org/10.3390/su11061765

Ambrosino, D. \& Sciomachen, A. (2021). Impact of externalities on the design and management of multimodal logistic networks. Sustainability, 13(9), 5080. https://doi.org/10.3390/su13095080

Ameknassi, L., Aït-Kadi, D. \& Rezg, N. (2016). Integration of logistics outsourcing decisions in a green supply chain design: A stochastic multi-objective multi-period multi-product programming model. International 
Journal of Production Economics, 182, 165-184. https://doi.org/10.1016/j.ijpe.2016.08.031

Amézquita-López, J., Agámez-Arias, A. \& Jurado-Almonte, J. (2018). La infraestructura logística en el sur de Bolívar: Estado actual y potencialidades. In J. A. Márquez Domínguez (Ed.), Sistemas productivos con anclaje territorial (pp. 311-325).

Ansari, S., Başdere, M., Li, X., Ouyang, Y. \& Smilowitz, K. (2018). Advancements in continuous approximation models for logistics and transportation systems: 1996-2016. Transportation Research Part B: Methodological, 107, 229-252. https://doi.org/10.1016/j.trb.2017.09.019

Anvari, S. \& Turkay, M. (2017). The facility location problem from the perspective of triple bottom line accounting of sustainability. International Journal of Production Research, 55(21), 6266-6287. https://doi.org/10.1080/00207543.2017.1341064

Arabani, A. \& Farahani, R. (2012). Facility location dynamics: An overview of classifications and applications. Computers \& Industrial Engineering, 62(1), 408-420. https://doi.org/10.1016/j.cie.2011.09.018

Arampantzi, C. \& Minis, I. (2017). A new model for designing sustainable supply chain networks and its application to a global manufacturer. Journal of Cleaner Production, 156, 276-292. https://doi.org/10.1016/j.jclepro.2017.03.164

Archer, N. \& Ghasemzadeh, F. (1999). An integrated framework for project portfolio selection. International Journal of Project Management, 17(4), 207-216. https://doi.org/10.1016/S0263-7863(98)00032-5

Arnold, P., Peeters, D. \& Thomas, I. (2004). Modelling a rail/road intermodal transportation system. Transportation Research Part E: Logistics and Transportation Review, 40(3), 255-270. https://doi.org/10.1016/j.tre.2003.08.005

Asl, A., Zarandi, F., Sotudian, S. \& Amini, A. (2020). A fuzzy capacitated facility location-network design model: A hybrid firefly and invasive weed optimization (FIWO) solution. Iranian Journal of Fuzzy Systems, 17(2), 79-95. https://doi.org/10.22111/ijfs.2020.5221

Assadipour, G., Ke, G. \& Verma, M. (2016). A toll-based bi-level programming approach to managing hazardous materials shipments over an intermodal transportation network. Transportation Research Part D: Transport and Environment, 47, 208-221. https://doi.org/10.1016/j.trd.2016.06.002

Ayar, B. \& Yaman, H. (2012). An intermodal multicommodity routing problem with scheduled services. Computational Optimization and Applications, 53(1), 131-153. https://doi.org/10.1007/s10589-011-9409-z

Badri, H., Bashiri, M. \& Hejazi, T. (2013). Integrated strategic and tactical planning in a supply chain network design with a heuristic solution method. Computers \& Operations Research, 40(4), 1143-1154. https://doi.org/10.1016/j.cor.2012.11.005

Badyal, V., Ferrell, W., Huynh, N. \& Padmanabhan, B. (2020). Multi-Period Optimization Model for Siting Capacitated Intermodal Facilities. Transportation Research Record, 2674(7), 135-147. https://doi.org/10.1177/0361198120921165

Bagloee, S., Sarvi, M., Patriksson, M. \& Asadi, M. (2018). Optimization for roads' construction: Selection, prioritization, and scheduling. Computer-Aided Civil and Infrastructure Engineering, 33(10), 833-848. https://doi.org/10.1111/mice.12370

Bagloee, S. \& Tavana, M. (2012). An efficient hybrid heuristic method for prioritising large transportation projects with interdependent activities. International Journal of Logistics Systems and Management, 11(1), 114-142. https://doi.org/10.1504/IJLSM.2012.044052

Bal, A. \& Satoglu, S. (2018). A goal programming model for sustainable reverse logistics operations planning and an application. Journal of Cleaner Production, 201, 1081-1091. https://doi.org/10.1016/j.jclepro.2018.08.104

Barbosa-Póvoa, A., da Silva, C. \& Carvalho, A. (2018). Opportunities and challenges in sustainable supply chain: An operations research perspective. European Journal of Operational Research, 268(2), 399-431. https://doi.org/10.1016/j.ejor.2017.10.036

Baykasoğlu, A. \& Gölcük, İ. (2019). A dynamic multiple attribute decision making model with learning of fuzzy cognitive maps. Computers \& Industrial Engineering, 135, 1063-1076. https://doi.org/10.1016/j.cie.2019.06.032

Baykasoğlu, A., Subulan, K., Taşan, A. \& Dudaklı, N. (2019). A review of fleet planning problems in single and multimodal transportation systems. Transportmetrica A: Transport Science, 15(2), 631-697. https://doi.org/10.1080/23249935.2018.1523249

Behbahani, H., Nazari, S., Partovifar, H. \& Kang, M. (2019). Designing a road network using John Rawls's social justice approach. Journal of Urban Planning and Development, 145(2), 05019002. https://doi.org/10.1061/(ASCE)UP.1943-5444.0000500 
Bendekovic, J., Rogozar, Z. \& Naletina, D. (2014). The role and importance of intermodal transport in Croatia for $\mathrm{EU}$ countries. In DAAAM International Scientific Book (pp. 267-278). https://doi.org/10.2507/daaam.scibook.2014.22

Berg, C., Deichmann, U., Liu, Y. \& Selod, H. (2017). Transport policies and development. The Journal of Development Studies, 53(4), 465-480. https://doi.org/10.1080/00220388.2016.1199857

Bertolini, L. \& le Clercq, F. (2003). Urban development without more mobility by car? Lessons from Amsterdam, a multimodal urban region. Environment and Planning A: Economy and Space, 35(4), 575-589. https://doi.org/10.1068/a3592

Bhattacharyya, R., Kumar, P. \& Kar, S. (2011). Fuzzy R\&D portfolio selection of interdependent projects. $\begin{array}{lllll}\text { Computers } \& \text { Mathematics } & \text { Applications, }\end{array}$ https://doi.org/10.1016/j.camwa.2011.09.036

Bhattarai, S., Golias, M., Mishra, S. \& Talebian, A. (2020). Multidimensional resource allocation for freight transportation project planning and decision making. Transportation Research Part A: Policy and Practice, 137, 95-110. https://doi.org/10.1016/j.tra.2020.04.017

Biermann, F., Teuber, M. \& Wedemeier, J. (2015). Bremen's and Hamburg's port position: Transport infrastructure and hinterland connections within the North Range. International Business and Global Economy, 34(1), 78-89. https://doi.org/10.4467/23539496IB.13.006.3979

Bigotte, J., Krass, D., Antunes, A. \& Berman, O. (2010). Integrated modeling of urban hierarchy and transportation network planning. Transportation Research Part A: Policy and Practice, 44(7), 506-522. https://doi.org/10.1016/j.tra.2010.03.020

Bolouri, S., Vafaeinejad, A., Alesheikh, A. \& Aghamohammadi, H. (2018). The ordered capacitated multiobjective location-allocation problem for fire stations using spatial optimization. ISPRS International Journal of Geo-Information, 7(2), 44. https://doi.org/10.3390/ijgi7020044

Bontekoning, Y., Macharis, C. \& Trip, J. (2004). Is a new applied transportation research field emerging?-A review of intermodal rail-truck freight transport literature. Transportation Research Part A: Policy and Practice, 38(1), 1-34. https://doi.org/10.1016/j.tra.2003.06.001

Bouzembrak, Y., Allaoui, H., Goncalves, G. \& Bouchriha, H. (2013). A multi-modal supply chain network design for recycling waterway sediments. International Journal of Environment and Pollution, 51(1/2), 15-31. https://doi.org/10.1504/IJEP.2013.053176

Bradley, S., Hax, A. \& Magnanti, T. (1977). Applied Mathematical Programming (Addison-Wesley (ed.)). http://web.mit.edu/15.053/www/

Braekers, K., Caris, A. \& Janssens, G. (2013). Integrated planning of loaded and empty container movements. $O R$ Spectrum, 35(2), 457-478. https://doi.org/10.1007/s00291-012-0284-5

Brahami, M., Dahane, M., Souier, M. \& Sahnoun, M. (2020). Sustainable capacitated facility location/network design problem: A non-dominated sorting genetic algorithm based multiobjective approach. Annals of Operations Research. https://doi.org/10.1007/s10479-020-03659-9

Bravo, J. \& Vidal, C. (2013). Freight transportation function in supply chain optimization models: A critical review of recent trends. Expert Systems with Applications, 40(17), 6742-6757. https://doi.org/10.1016/j.eswa.2013.06.015

Broniewicz, E. \& Ogrodnik, K. (2020). Multi-criteria analysis of transport infrastructure projects. Transportation Research Part D: Transport and Environment, 83, 102351. https://doi.org/10.1016/j.trd.2020.102351

Bubicz, M, Barbosa-Póvoa, A. \& Carvalho, A. (2019). Incorporating social aspects in sustainable supply chains: Trends and future directions. Journal of Cleaner Production, 237, 117500. https://doi.org/10.1016/j.jclepro.2019.06.331

Camargo-Pérez, J., Montoya-Torres, J. \& Carrillo-Ramirez, M. (2013). Multi-criteria deision making for locating multimodal transfer nodes in passenger transport systems. 2013 IEEE International Conference on Systems, Man, and Cybernetics, 718-723. https://doi.org/10.1109/SMC.2013.127

Campbell, J. \& O'Kelly, M. (2012). Twenty-five years of hub location research. Transportation Science, 46(2), 153-169. https://doi.org/10.1287/trsc.1120.0410

Caris, A., Limbourg, S., Macharis, C., van Lier, T. \& Cools, M. (2014). Integration of inland waterway transport in the intermodal supply chain: A taxonomy of research challenges. Journal of Transport Geography, 41, 126136. https://doi.org/10.1016/j.jtrangeo.2014.08.022

Caris, A., Macharis, C. \& Janssens, G. (2008). Planning problems in intermodal freight transport: Accomplishments and prospects. Transportation Planning and Technology, 31(3), $277-302$. https://doi.org/10.1080/03081060802086397 
Caris, A., Macharis, C. \& Janssens, G. (2013). Decision support in intermodal transport: A new research agenda. Computers in Industry, 64(2), 105-112. https://doi.org/10.1016/j.compind.2012.12.001

Carpio-Pinedo, J. (2021). Multimodal transport and potential encounters with social difference: A novel approach based on network analysis. Journal of Urban Affairs, 43(1), 93-116. https://doi.org/10.1080/07352166.2019.1662727

Casu, M., Cicala, G. \& Tacchella, A. (2013). Ontology-based data access: An application to intermodal logistics. Information Systems Frontiers, 15(5), 849-871. https://doi.org/10.1007/s10796-012-9395-4

Chen, A., Zhou, Z., Chootinan, P., Ryu, S., Yang, C. \& Wong, S. (2011). Transport network design problem under uncertainty: A review and new developments. Transport Reviews, 31(6), 743-768. https://doi.org/10.1080/01441647.2011.589539

Cho, J., Kim, H. \& Choi, H. (2012). An intermodal transport network planning algorithm using dynamic programming - A case study: From Busan to Rotterdam in intermodal freight routing. Applied Intelligence, 36(3), 529-541. https://doi.org/10.1007/s10489-010-0223-6

Chopra, S. \& Meindl, P. (2013). Supply chain management: strategy, planning, and operation. In Supply chain management: strategy, planning, and operation (Pearson).

Comisión de la Verdad. (2020). https://comisiondelaverdad.co/actualidad/blogs/en-medio-de-la-presion-deldesplazamiento-forzado-el-magdalena-medio-resiste-y-permanece

Consejo Privado de Competitividad de Colombia. (2021). Índice Departamental de Competitividad. https://compite.com.co/indice-departamental-de-competitividad/

Contreras, I. \& Fernández, E. (2012). General network design: A unified view of combined location and network design problems. European Journal of Operational Research, 219(3), 680-697. https://doi.org/10.1016/j.ejor.2011.11.009

Cordeau, J., Pasin, F. \& Solomon, M. (2006). An integrated model for logistics network design. Annals of Operations Research, 144(1), 59-82. https://doi.org/10.1007/s10479-006-0001-3

Cordier, R., Milbourn, B., Martin, R., Buchanan, A., Chung, D. \& Speyer, R. (2017). A systematic review evaluating the psychometric properties of measures of social inclusion. PLOS ONE, 12(6), e0179109. https://doi.org/10.1371/journal.pone.0179109

Cornet, Y., Barradale, M., Gudmundsson, H. \& Barfod, M. (2018). Engaging multiple actors in large-scale transport infrastructure project appraisal: An application of MAMCA to the case of HS2 high-speed rail. Journal of Advanced Transportation, 2018, 1-22. https://doi.org/10.1155/2018/9267306

Coronado-Mondragon, A., Lalwani, C., Coronado-Mondragon, E., Coronado-Mondragon, C. \& Pawar, K. (2012). Intelligent transport systems in multimodal logistics: A case of role and contribution through wireless vehicular networks in a sea port location. International Journal of Production Economics, 137(1), 165-175. https://doi.org/10.1016/j.ijpe.2011.11.006

Corporación Autónoma Regional del Río Grande de la Magdalena. (17 de marzo de 2021). Plan de acción 20192021: Por la transparencia y la eficiencia. http://www.cormagdalena.gov.co/

Corredor, L. \& Díaz, L. (2018). Navegabilidad del Río Magdalena y competitividad de la logística del transporte en Colombia. Questionar: Investigación Específica, 6(1), 67-78. https://doi.org/10.29097/23461098.249

Correia, I. \& Melo, T. (2016). Multi-period capacitated facility location under delayed demand satisfaction. European Journal of Operational Research, 255(3), 729-746. https://doi.org/10.1016/j.ejor.2016.06.039

Correia, I. \& Melo, T. (2017). A multi-period facility location problem with modular capacity adjustments and flexible demand fulfillment. Computers \& Industrial Engineering, 110, 307-321. https://doi.org/10.1016/j.cie.2017.06.003

Correia, I., Nickel, S. \& Saldanha-da-Gama, F. (2010). Single-assignment hub location problems with multiple capacity levels. Transportation Research Part B: Methodological, 44(8-9), 1047-1066. https://doi.org/10.1016/j.trb.2009.12.016

Correia, I., Nickel, S. \& Saldanha-da-Gama, F. (2018). A stochastic multi-period capacitated multiple allocation hub location problem: Formulation and inequalities. Omega, 74, 122-134. https://doi.org/10.1016/j.omega.2017.01.011

Corry, P. \& Kozan, E. (2008). Optimised loading patterns for intermodal trains. OR Spectrum, 30(4), 721-750. https://doi.org/10.1007/s00291-007-0112-5

Crainic, T. (2000). Service network design in freight transportation. European Journal of Operational Research, 122(2), 272-288. https://doi.org/10.1016/S0377-2217(99)00233-7

Crainic, T. \& Kim, K. (2007). Chapter 8 Intermodal Transportation. In Handbooks in Operations Research and Management Science (Vol. 14, pp. 467-537). https://doi.org/10.1016/S0927-0507(06)14008-6 
Crainic, T. \& Laporte, G. (1997). Planning models for freight transportation. European Journal of Operational Research, 97(3), 409-438. https://doi.org/10.1016/S0377-2217(96)00298-6

Crainic, T., Perboli, G. \& Rosano, M. (2018). Simulation of intermodal freight transportation systems: A taxonomy. European Journal of Operational Research, 270(2), 401-418. https://doi.org/10.1016/j.ejor.2017.11.061

D’Eusanio, M., Zamagni, A. \& Petti, L. (2019). Social sustainability and supply chain management: Methods and tools. Journal of Cleaner Production, 235, 178-189. https://doi.org/10.1016/j.jclepro.2019.06.323

Danesh, D., Ryan, M. \& Abbasi, A. (2018). Multi-criteria decision-making methods for project portfolio management: A literature review. International Journal of Management and Decision Making, 16(1), 75-94. https://doi.org/10.1504/IJMDM.2017.10006139

Dantzig, G. (1963). Linear programming and extensions. In R. Corporation (Ed.), Linear Programming and Extensions (Issue August).

Darabann, S., Stefanescu, P. \& Crisan, R. (2012). Economic benefits of developing intermodal transport in the European Union. Annals of the University of Oradea, Economic Science Series, 1(2), 81-87. https://ideas.repec.org/a/ora/journl/v1y2012i2p81-87.html

Davidson, E. \& Leachman, R. (2012). Optimizing a multi-strategy port and modal allocation of containerized imports from asia to the United States. Journal of Marketing Channels, 19(2), 120-140. https://doi.org/10.1080/1046669X.2012.667762

Dekker, R., Bloemhof, J. \& Mallidis, I. (2012). Operations research for green logistics - An overview of aspects, issues, contributions and challenges. European Journal of Operational Research, 219(3), 671-679. https://doi.org/10.1016/j.ejor.2011.11.010

Dekker, R., van Asperen, E., Ochtman, G. \& Kusters, W. (2009). Floating stocks in FMCG supply chains: using intermodal transport to facilitate advance deployment. International Journal of Physical Distribution \& Logistics Management, 39(8), 632-648. https://doi.org/10.1108/09600030910996297

Delbart, T., Molenbruch, Y., Braekers, K. \& Caris, A. (2021). Uncertainty in Intermodal and Synchromodal Transport: Review and Future Research Directions. Sustainability, 13(7), 3980. https://doi.org/10.3390/su13073980

Denyer, D. \& Tranfield, D. (2009). Producing a systematic review. In The sage handbook of organizational reseacrh methods (pp. 671-689).

Departamento Administrativo Nacional de Estadísticas (30 de abril de 2021). Geoportal DANE. https://geoportal.dane.gov.co/

Departamento Nacional de Planeación. (26 de noviembre de 2020). Plan Maestro Ferroviario. https://colaboracion.dnp.gov.co/CDT/Prensa/Plan-Maestro-Ferroviario.pdf

Departamento Nacional de Planeación. (5 de mayo de 2021). CONPES 3982: Política Nacional Logística. https://colaboracion.dnp.gov.co/CDT/Conpes/Econ\%C3\%B3micos/3982.pdf

Departamento Nacional de Planeación. (7 de agosto de 2019). Pacto por Colombia, pacto por la equidad. https://colaboracion.dnp.gov.co/CDT/Prensa/Resumen-PND2018-2022-final.pdf

Dewan, I., Zunder, T. \& Jorna, R. (2013). Performance evaluation of an online benchmarking tool for European freight transport chains. Benchmarking: An International Journal, 20(2), 233-250. https://doi.org/10.1108/14635771311307696

Díaz, D. (2020). Avances y retos de la Política Nacional Logística. https://grupozfb.com/wpcontent/uploads/2020/09/AVANCES-Y-RETOS-DE-LA-PNL.pdf

Ding, Z., Lu, Y., Lai, K., Yang, M. \& Lee, W. (2020). Optimal coordinated operation scheduling for electric vehicle aggregator and charging stations in an integrated electricity-transportation system. International Journal of Electrical Power \& Energy Systems, 121, 106040. https://doi.org/10.1016/j.ijepes.2020.106040

Dotoli, M., Zgaya, H., Russo, C. \& Hammadi, S. (2017). A multi-agent advanced traveler information system for optimal trip planning in a co-modal framework. IEEE Transactions on Intelligent Transportation Systems, 18(9), 2397-2412. https://doi.org/10.1109/TITS.2016.2645278

Dua, A. \& Sinha, D. (2019). Quality of multimodal freight transportation: a systematic literature review. World Review of Intermodal Transportation Research, 8(2), 167. https://doi.org/10.1504/WRITR.2019.099136

Dukkanci, O., Peker, M. \& Kara, B. (2019). Green hub location problem. Transportation Research Part E: Logistics and Transportation Review, 125, 116-139. https://doi.org/10.1016/j.tre.2019.03.005

Dunning, I., Huchette, J. \& Lubin, M. (2017). JuMP: A modeling language for mathematical optimization. SIAM Review, 59(2), 295-320. https://doi.org/10.1137/15M1020575 
Durango-Cohen, P. \& Sarutipand, P. (2007). Capturing interdependencies and heterogeneity in the management of multifacility transportation infrastructure systems. Journal of Infrastructure Systems, 13(2), 115-123. https://doi.org/10.1061/(ASCE)1076-0342(2007)13:2(115)

Elbert, R., Müller, J. \& Rentschler, J. (2020). Tactical network planning and design in multimodal transportation - A systematic literature review. Research in Transportation Business \& Management, 35, 100462. https://doi.org/10.1016/j.rtbm.2020.100462

Eskandarpour, M., Dejax, P., Miemczyk, J. \& Péton, O. (2015). Sustainable supply chain network design: An optimization-oriented review. Omega, 54, 11-32. https://doi.org/10.1016/j.omega.2015.01.006

Esteso, A., Alemany, M., Ortiz, Á. \& Peidro, D. (2018). A multi-objective model for inventory and planned production reassignment to committed orders with homogeneity requirements. Computers \& Industrial Engineering, 124(May), 180-194. https://doi.org/10.1016/j.cie.2018.07.025

Evers, P. \& Johnson, C. (2000). Performance perceptions, satisfaction, and intention: The intermodal shipper's perspective. Transportation Journal, 40(2), 27-39. http://www.jstor.org/stable/20713452

Farahani, R. \& Hekmatfar, M. (2009). Facility Location. In R. Zanjirani Farahani \& M. Hekmatfar (Eds.), Facility Location: Concepts, Models, Algorithms and Case Studies (First, Vol. 40, Issue 3). Physica-Verlag HD. https://doi.org/10.1007/978-3-7908-2151-2

Farahani, R., Hekmatfar, M., Arabani, A. \& Nikbakhsh, E. (2013). Hub location problems: A review of models, classification, solution techniques, and applications. Computers and Industrial Engineering, 64(4), 10961109. https://doi.org/10.1016/j.cie.2013.01.012

Farahani, R., Miandoabchi, E., Szeto, W. \& Rashidi, H. (2013). A review of urban transportation network design problems. European Journal of Operational Research, 229(2), 281-302. https://doi.org/10.1016/j.ejor.2013.01.001

Farahani, R., SteadieSeifi, M. \& Asgari, N. (2010). Multiple criteria facility location problems: A survey. Applied Mathematical Modelling, 34(7), 1689-1709. https://doi.org/10.1016/j.apm.2009.10.005

Fathollahi-Fard, A. \& Hajaghaei-Keshteli, M. (2018). A tri-level location-allocation model for forward/reverse supply chain. Applied Soft Computing, 62, 328-346. https://doi.org/10.1016/j.asoc.2017.11.004

Fattahi, M., Mahootchi, M., Govindan, K. \& Moattar Husseini, S. (2015). Dynamic supply chain network design with capacity planning and multi-period pricing. Transportation Research Part E: Logistics and Transportation Review, 81, 169-202. https://doi.org/10.1016/j.tre.2015.06.007

Fernandes, L., Relvas, S. \& Barbosa-Póvoa, A. (2013). Strategic network design of downstream petroleum supply chains: Single versus multi-entity participation. Chemical Engineering Research and Design, 91(8), 15571587. https://doi.org/10.1016/j.cherd.2013.05.028

Flórez, S. (31 de diciembre 2018). La deforestación en Bolívar, un enemigo silencioso. El Universal. https://www.eluniversal.com.co/regional/deforestacion-ha-en-el-ano-2017-en-los-municipios-de-bolivarJD533748

Fotuhi, F. \& Huynh, N. (2018). A reliable multi-period intermodal freight network expansion problem. Computers \& Industrial Engineering, 115, 138-150. https://doi.org/10.1016/j.cie.2017.11.007

Garay-Sianca, A. \& Nurre-Pinkley, S. (2021). Interdependent integrated network design and scheduling problems with movement of machines. European Journal of Operational Research, 289(1), 297-327. https://doi.org/10.1016/j.ejor.2020.07.013

García-Montero, L., López, E., Monzón, A. \& Otero, I. (2010). Environmental screening tools for assessment of infrastructure plans based on biodiversity preservation and global warming (PEIT, Spain). Environmental Impact Assessment Review, 30(3), 158-168. https://doi.org/10.1016/j.eiar.2009.08.008

García-Sabater, J. \& Maheut, J. (2015). Modelado y resolución de problemas de organización industrial mediante programación matemática http://personales.upv.es/jpgarcia/LinkedDocuments/modeladomatematico.pdf

García-Sabater, J. (2021). Programación matemática en Python con PULP. http://hdl.handle.net/10251/158416

García-Sánchez, J. (2012). Modelling in mathematical programming: Methodology and techniques. Springer International Publishing. https://doi.org/10.1007/978-3-030-57250-1

Ghaderi, A. \& Jabalameli, M. (2013). Modeling the budget-constrained dynamic uncapacitated facility locationnetwork design problem and solving it via two efficient heuristics: A case study of health care. Mathematical and Computer Modelling, 57(3-4), 382-400. https://doi.org/10.1016/j.mcm.2012.06.017

Giannopoulos, G. (2004). The application of information and communication technologies in transport. European Journal of Operational Research, 152(2), 302-320. https://doi.org/10.1016/S0377-2217(03)00026-2

Ginieis, M., Sánchez-Rebull, M. \& Campa-Planas, F. (2012). The academic journal literature on air transport: 
Analysis using systematic literature review methodology. Journal of Air Transport Management, 19(1), 3135. https://doi.org/10.1016/j.jairtraman.2011.12.005

Gobernación de Bolívar. (2016). Plan de desarrollo del departamento de Bolívar 2016-2019.

Gobernación de Bolívar. (2020a). Plan de desarrollo del departamento de Bolívar 2020-2023.

Gobernación de Bolívar. (2020b). Plan departamental de extensión agropeuaría 2020-2023.

Govindan, K., Fattahi, M. \& Keyvanshokooh, E. (2017). Supply chain network design under uncertainty: A comprehensive review and future research directions. European Journal of Operational Research, 263(1), 108-141. https://doi.org/10.1016/j.ejor.2017.04.009

Guimarães, V., Ribeiro, G., Forte, V. \& Lucena, A. (2017). A location-allocation model for Logistics integration centers. International Journal of Transport Economics, 44(2), 273-292. https://doi.org/10.19272/201706702006

Guo, Y., Xia, D., Sun, B., Wang, X., Zhang, D. \& Li, J. (2018). Ecological footprints and development trends in Hefei, China. Management of Environmental Quality: An International Journal, 29(1), 2-14. https://doi.org/10.1108/MEQ-12-2016-0094

Gurobi Optimización (2019). Manual de referencia del optimizador Gurobi, version 8.1. https://www.gurobi.com/documentation/8.1/refman/refman.html

Hajibabai, L. \& Ouyang, Y. (2013). Integrated Planning of Supply Chain Networks and Multimodal Transportation Infrastructure Expansion: Model Development and Application to the Biofuel Industry. Computer-Aided Civil and Infrastructure Engineering, 28(4), 247-259. https://doi.org/10.1111/j.14678667.2012.00791.x

Harris, I., Wang, Y. \& Wang, H. (2015). ICT in multimodal transport and technological trends: Unleashing potential for the future. International Journal of Production Economics, 159, 88-103. https://doi.org/10.1016/j.ijpe.2014.09.005

Hasan, K., Zhang, W. \& Shi, W. (2021). Barriers to intermodal freight diversion: a total logistics cost approach. Maritime Economics \& Logistics. https://doi.org/10.1057/s41278-020-00182-3

Hernández, S., \& Monzón, A. (2016). Key factors for defining an efficient urban transport interchange: Users' perceptions. Cities, 50, 158-167. https://doi.org/10.1016/j.cities.2015.09.009

Higuera, E. (2020). Logística, competitividad y zonas francas. https://grupozfb.com/wpcontent/uploads/2020/09/Logistica-Zonas-Francas-2020-Edgar-H.pdf

Hoff, A., Peiró, J., Corberán, Á. \& Martí, R. (2017). Heuristics for the capacitated modular hub location problem. Computers \& Operations Research, 86, 94-109. https://doi.org/10.1016/j.cor.2017.05.004

Hosseininasab, S. \& Shetab-Boushehri, S. (2015). Integration of selecting and scheduling urban road construction projects as a time-dependent discrete network design problem. European Journal of Operational Research, 246(3), 762-771. https://doi.org/10.1016/j.ejor.2015.05.039

Hosseininasab, S., Shetab-Boushehri, S., Hejazi, S. \& Karimi, H. (2018). A multi-objective integrated model for selecting, scheduling, and budgeting road construction projects. European Journal of Operational Research, 271(1), 262-277. https://doi.org/10.1016/j.ejor.2018.04.051

Instituto de Hidrología, Meteorología y Estudios Ambientales. (24 de abril de 2021). Monitoreo del cambio en la superficie cubierta por bosque natural. http://www.ideam.gov.co/web/ecosistemas/cambio-de-la-superficiecubierta-por-bosque-natural

Instituto Nacional de Vías. (7 de mayo de 2021a). Clasificación de las carreteras en Colombia. https://www.invias.gov.co/index.php/red-vial-nacional/2-uncategorised/2706-clasificacion-de-las-carreteras

Instituto Nacional de Vías. (15 de junio de 2021b). Mapas de la red vial. https://www.invias.gov.co/index.php/component/content/article/2-uncategorised/59-mapas-de-la-red terciaria-y-ferrea

Ishfaq, R. \& Sox, C. (2012). Design of intermodal logistics networks with hub delays. European Journal of Operational Research, 220(3), 629-641. https://doi.org/10.1016/j.ejor.2012.03.010

Isler, C., Asaff, Y. \& Marinov, M. (2020). Designing a geo-strategic railway freight network in Brazil using GIS. Sustainability, 13(1), 85. https://doi.org/10.3390/su13010085

Jabalameli, M. \& Mortezaei, M. (2011). A hybrid model for multi-objective capacitated facility location network design problem. International Journal of Industrial Engineering Computations, 2(3), 509-522. https://doi.org/10.5267/j.ijiec.2011.04.001

Jamshidi, A., Jamshidi, F., Ait-Kadi, D. \& Ramudhin, A. (2019). A review of priority criteria and decision-making methods applied in selection of sustainable city logistics initiatives and collaboration partners. International 
Journal of Production Research, 57(15-16), 5175-5193. https://doi.org/10.1080/00207543.2018.1540892

Jawad, S. \& Liu, J. (2020). Electrical vehicle charging services planning and operation with interdependent power networks and transportation networks: A review of the current scenario and future trends. Energies, 13(13), 3371. https://doi.org/10.3390/en13133371

Jena, S., Cordeau, J. \& Gendron, B. (2015). Dynamic facility location with generalized modular capacities. Transportation Science, 49(3), 484-499. https://doi.org/10.1287/trsc.2014.0575

Jeon, C. \& Amekudzi, A. (2005). Addressing sustainability in transportation systems: Definitions, indicators, and metrics. Journal of Infrastructure Systems, 11(1), 31-50. https://doi.org/10.1061/(ASCE)10760342(2005)11:1(31)

Jia, G., Ma, R. \& Hu, Z. (2019). Review of urban transportation network design problems based on CiteSpace. Mathematical Problems in Engineering, 2019, 1-22. https://doi.org/10.1155/2019/5735702

Josa, I. \& Aguado, A. (2019). Infrastructures and society: From a literature review to a conceptual framework. Journal of Cleaner Production, 238, 117741. https://doi.org/10.1016/j.jclepro.2019.117741

Jovovic, R., Draskovic, M., Delibasic, M. \& Jovovic, M. (2017). The concept of sustainable regional development - institutional aspects, policies and prospects. Journal of International Studies, 10(1), 255-266. https://doi.org/10.14254/2071-8330.2017/10-1/18

Kadaifci, C., Asan, U., Serdarasan, S. \& Arican, U. (2019). A new rule-based integrated decision making approach to container transshipment terminal selection. Maritime Policy \& Management, 46(2), 237-256. https://doi.org/10.1080/03088839.2018.1489149

Kasso, M. \& Bekele, A. (2018). Post-harvest loss and quality deterioration of horticultural crops in Dire Dawa Region, Ethiopia. Journal of the Saudi Society of Agricultural Sciences, 17(1), 88-96. https://doi.org/10.1016/j.jssas.2016.01.005

Kazemi, M., Sedighizadeh, M., Mirzaei, M. \& Homaee, O. (2016). Optimal siting and sizing of distribution system operator owned EV parking lots. Applied Energy, 179, 1176-1184. https://doi.org/10.1016/j.apenergy.2016.06.125

Kazemi, Y. \& Szmerekovsky, J. (2015). Modeling downstream petroleum supply chain: The importance of multimode transportation to strategic planning. Transportation Research Part E: Logistics and Transportation Review, 83, 111-125. https://doi.org/10.1016/j.tre.2015.09.004

Kelleher, G., El-Rhalibi, A. \& Arshad, F. (2003). Scheduling for intermodal transport. Logistics Information Management, 16(5), 363-372. https://doi.org/10.1108/09576050310499354

Khaslavskaya, A. \& Roso, V. (2020). Dry ports: research outcomes, trends, and future implications. Maritime Economics \& Logistics, 22(2), 265-292. https://doi.org/10.1057/s41278-020-00152-9

Khodakarami, M., Mitchell, K. \& Wang, X. (2014). Modeling maintenance project selection on a multimodal transportation network. Transportation Research Record, 2409(1), 1-8. https://doi.org/10.3141/2409-01

Khooban, Z. (2011). Transportation. In Logistics Operations and Management (pp. 109-126). Elsevier. https://doi.org/10.1016/B978-0-12-385202-1.00007-4

Kim, S., Park, M. \& Lee, C. (2013). Multimodal freight transportation network design problem for reduction of greenhouse gas emissions. Transportation Research Record, 2340(1), 74-83. https://doi.org/10.3141/2340-09

Klose, A. \& Drexl, A. (2005). Facility location models for distribution system design. European Journal of Operational Research, 162(1), 4-29. https://doi.org/10.1016/j.ejor.2003.10.031

Ko, S., Lautala, P., Fan, J. \& Shonnard, D. (2019). Economic, social, and environmental cost optimization of biomass transportation: a regional model for transportation analysis in plant location process. Biofuels Bioproducts \& Biorefining-Biofpr, 13(3), 582-598. https://doi.org/10.1002/bbb.1967

Konstantinidou, M., Kepaptsoglou, K. \& Stathopoulos, A. (2019). A multi-objective network design model for post-disaster transportation network management. PROMET - Traffic\&Transportation, 31(1), 11-23. https://doi.org/10.7307/ptt.v31i1.2743

Kreutzberger, E. (2008). Distance and time in intermodal goods transport networks in Europe: A generic approach. Transportation Research Part A: Policy and Practice, 42(7), 973-993. https://doi.org/10.1016/j.tra.2008.01.012

Kumar, A. \& Anbanandam, R. (2019). Multimodal freight transportation strategic network design for sustainable supply chain. International Journal of System Dynamics Applications, 8(2), 19-35. https://doi.org/10.4018/IJSDA.2019040102

Kumar, A. \& Mishra, S. (2018). A simplified framework for sequencing of transportation projects considering user costs and benefits. Transportmetrica A: Transport Science, 14(4), 346-371. https://doi.org/10.1080/23249935.2017.1387827 
Kumar, R. (2008). Research methodology. (APH Publishing Corporation (Ed.)).

Lal, R. (2015). Restoring soil quality to mitigate soil degradation. Sustainability, 7(5), 5875-5895. https://doi.org/10.3390/su7055875

Lall, S., Schroeder, E. \& Schmidt, E. (2014). Identifying spatial efficiency-equity trade-offs in territorial development policies: Evidence from Uganda. The Journal of Development Studies, 50(12), 1717-1733. https://doi.org/10.1080/00220388.2014.957277

Lam, J. \& Gu, Y. (2013). Port hinterland intermodal container flow optimisation with green concerns: A literature review and research agenda. International Journal of Shipping and Transport Logistics, 5(3), 257. https://doi.org/10.1504/IJSTL.2013.054190

Lee, C. (1991). An optimal algorithm for the multiproduct capacitated facility location problem with a choice of facility type. Computers and Operations Research, 18(2), 167-182. https://doi.org/10.1016/03050548(91)90087-8

Leibowicz, B. (2020). Urban land use and transportation planning for climate change mitigation: A theoretical framework. European Journal of Operational Research, 284(2), 604-616. https://doi.org/10.1016/j.ejor.2019.12.034

Leviakangas, P., Haajanen, J. \& Alaruikka, A. (2007). Information service architecture for international multimodal logistic corridor. IEEE Transactions on Intelligent Transportation Systems, 8(4), 565-574. https://doi.org/10.1109/TITS.2007.903049

Li, Z., Kaul, H., Kapoor, S., Veliou, E., Zhou, B. \& Lee, S. (2012). New methodology for transportation investment decisions with consideration of project interdependencies. Transportation Research Record, 2285(1), 36-46. https://doi.org/10.3141/2285-05

Litman, T. (2007). Developing indicators for comprehensive and sustainable transport planning. Transportation Research Record, 2017(1), 10-15. https://doi.org/10.3141/2017-02

Litman T. (2019). Evaluating transportation equity: Guidance for incorporating distributional impacts in transportation planning. Victoria Transport Policy Institute, págs. 66.

Liu, X. \& Kwon, C. (2020). Exact robust solutions for the combined facility location and network design problem in hazardous materials transportation. IISE Transactions, 52(10), 1156-1172.

López-Ospina, H., Agudelo-Bernal, Á., Reyes-Muñoz, L., Zambrano-Rey, G. \& Pérez, J. (2021). Design of a location and transportation optimization model including quality of service using constrained multinomial logit. Applied Mathematical Modelling, 89(Part 1), 428-453. https://doi.org/10.1016/j.apm.2020.07.054

Lun, Y., Wong, C., Lai, K. \& Cheng, T. (2008). Institutional perspective on the adoption of technology for the security enhancement of container transport. Transport Reviews, 28(1), 21-33. https://doi.org/10.1080/01441640701358804

Macharis, C. \& Bontekoning, Y. (2004). Opportunities for OR in intermodal freight transport research: A review. European Journal of Operational Research, 153(2), 400-416. https://doi.org/10.1016/S0377-2217(03)001619

Macharis, C., de Witte, A. \& Ampe, J. (2009). The multi-actor, multi-criteria analysis methodology (MAMCA) for the evaluation of transport projects: Theory and practice. Journal of Advanced Transportation, 43(2), 183202. https://doi.org/10.1002/atr.5670430206

Maiyar, L. \& Thakkar, J. (2019). Modelling and analysis of intermodal food grain transportation under hub disruption towards sustainability. International Journal of Production Economics, 217, 281-297. https://doi.org/10.1016/j.ijpe.2018.07.021

Mansouri, S., Lee, H. \& Aluko, O. (2015). Multi-objective decision support to enhance environmental sustainability in maritime shipping: A review and future directions. Transportation Research Part E: Logistics and Transportation Review, 78, 3-18. https://doi.org/10.1016/j.tre.2015.01.012

Marchet, G., Perotti, S. \& Mangiaracina, R. (2012). Modelling the impacts of ICT adoption for intermodal transportation. International Journal of Physical Distribution \& Logistics Management, 42(2), 110-127. https://doi.org/10.1108/09600031211219645

Marín, A., Álvarez, C., Giraldo, C. \& Uribe, S. (2018). Análisis multitemporal del paisaje en el Magdalena Medio en el periodo 1985-2011: Una ventana de interpretación de cambios históricos e implicaciones en la conectividad estructural. Cuadernos de Geografía: Revista Colombiana de Geografía, 27(1), 10-26. https://doi.org/10.15446/rcdg.v27n1.55783

Marler, R. \& Arora, J. (2010). The weighted sum method for multi-objective optimization: new insights. Structural and Multidisciplinary Optimization, 41(6), 853-862. https://doi.org/10.1007/s00158-009-0460-7

Marler, R. \& Arora, J. (2004). Survey of multi-objective optimization methods for engineering. Structural and 
Multidisciplinary Optimization, 26(6), 369-395. https://doi.org/10.1007/s00158-003-0368-6

Márquez, L. (2017). El ferrocarril colombiano: 4 temas recurrentes en la literatura. Estudios Gerenciales, 33, 187194. https://doi.org/10.1016/j.estger.2017.04.003

Martínez-López, A. \& Chica, M. (2020). Joint optimization of routes and container fleets to design sustainable intermodal chains in Chile. Sustainability, 12(6), 2221. https://doi.org/10.3390/su12062221

Marufuzzaman, M., Eksioglu, S., Li, X. \& Wang, J. (2014). Analyzing the impact of intermodal-related risk to the design and management of biofuel supply chain. Transportation Research Part E: Logistics and Transportation Review, 69, 122-145. https://doi.org/10.1016/j.tre.2014.06.008

Mathur, K. \& Solow, D. (1996). Investigación de operaciones: El arte de la toma de decisiones (P. Hall (Ed.)).

Mavrotas, G. (2009). Effective implementation of the $\varepsilon$-constraint method in multi-objective mathematical programming problems. Applied Mathematics and Computation, 213(2), 455-465. https://doi.org/10.1016/j.amc.2009.03.037

Maza-Ávila, F., Agámez-Arias, A. \& Pérez-González, M. (2015). Pertinencia de la infraestructura de transporte para la competitividad del sector agrícola de la ZODES Montes de María: Una aproximación. Saber, Ciencia y Libertad, 10(1), 161-177. https://doi.org/10.18041/2382-3240/saber.2015v10n1.922

Meemken, E. \& Qaim, M. (2018). Can private food standards promote gender equality in the small farm sector? Journal of Rural Studies, 58(May 2017), 39-51. https://doi.org/10.1016/j.jrurstud.2017.12.030

Melkote, S. \& Daskin, M. (2001). Capacitated facility location/network design problems. European Journal of Operational Research, 129(3), 481-495. https://doi.org/10.1016/S0377-2217(99)00464-6

Melo, M., Nickel, S. \& Saldanha-da-Gama, F. (2009). Facility location and supply chain management - A review. European Journal of Operational Research, 196(2), 401-412. https://doi.org/10.1016/j.ejor.2008.05.007

Meng, Q. \& Wang, X. (2011). Intermodal hub-and-spoke network design: Incorporating multiple stakeholders and multi-type containers. Transportation Research Part B: Methodological, 45(4), 724-742. https://doi.org/10.1016/j.trb.2010.11.002

Messmann, L., Zender, V., Thorenz, A. \& Tuma, A. (2020). How to quantify social impacts in strategic supply chain optimization: State of the art. Journal of Cleaner Production, 257, 120459. https://doi.org/10.1016/j.jclepro.2020.120459

Miraj, P., Berawi, M., Zagloel, T., Sari, M. \& Saroji, G. (2021). Research trend of dry port studies: a two-decade systematic review. Maritime Policy \& Management, 48(4), 563-582. https://doi.org/10.1080/03088839.2020.1798031

Miralinaghi, M., Seilabi, S., Chen, S., Hsu, Y. \& Labi, S. (2020). Optimizing the selection and scheduling of multi -class projects using a Stackelberg framework. European Journal of Operational Research, 286(2), 508-522. https://doi.org/10.1016/j.ejor.2020.03.051

Miret, C., Chazara, P., Montastruc, L., Negny, S. \& Domenech, S. (2016). Design of bioethanol green supply chain: Comparison between first and second generation biomass concerning economic, environmental and social criteria. Computers \& Chemical Engineering, 85, 16-35. https://doi.org/10.1016/j.compchemeng.2015.10.008

Mogale, D., Cheikhrouhou, N. \& Tiwari, M. (2020). Modelling of sustainable food grain supply chain distribution system: a bi-objective approach. International Journal of Production Research, 58(18), 5521-5544. https://doi.org/10.1080/00207543.2019.1669840

Mogale, D., Kumar, S. \& Tiwari, M. (2018). An MINLP model to support the movement and storage decisions of the Indian food grain supply chain. Control Engineering Practice, 70, 98-113. https://doi.org/10.1016/j.conengprac.2017.09.017

Mohammadi, M., Jula, P. \& Tavakkoli-Moghaddam, R. (2019). Reliable single-allocation hub location problem with disruptions. Transportation Research Part E: Logistics and Transportation Review, 123, 90-120. https://doi.org/10.1016/j.tre.2019.01.008

Mohammed, A., Harris, I., Soroka, A. \& Nujoom, R. (2019). A hybrid MCDM-fuzzy multi-objective programming approach for a G-resilient supply chain network design. Computers \& Industrial Engineering, 127, 297-312. https://doi.org/10.1016/j.cie.2018.09.052

Moheb-Alizadeh, H. \& Handfield, R. (2019). Sustainable supplier selection and order allocation: A novel multiobjective programming model with a hybrid solution approach. Computers \& Industrial Engineering, 129, 192-209. https://doi.org/10.1016/j.cie.2019.01.011

Monios, J. (2016). Intermodal transport as a regional development strategy: The case of italian freight villages. Growth and Change, 47(3), 363-377. https://doi.org/10.1111/grow.12129

Monios, J. \& Wilmsmeier, G. (2013). The role of intermodal transport in port regionalisation. Transport Policy, 
30, 161-172. https://doi.org/10.1016/j.tranpol.2013.09.010

Moreno-Camacho, C., Montoya-Torres, J., Jaegler, A. \& Gondran, N. (2019). Sustainability metrics for real case applications of the supply chain network design problem: A systematic literature review. Journal of Cleaner Production, 231, 600-618. https://doi.org/10.1016/j.jclepro.2019.05.278

Mostert, M., Caris, A. \& Limbourg, S. (2017). Road and intermodal transport performance: the impact of operational costs and air pollution external costs. Research in Transportation Business \& Management, 23, 75-85. https://doi.org/10.1016/j.rtbm.2017.02.004

Mota, B., Gomes, M., Carvalho, A. \& Barbosa-Povoa, A. (2015). Towards supply chain sustainability: economic, environmental and social design and planning. Journal of Cleaner Production, 105, 14-27. https://doi.org/10.1016/j.jclepro.2014.07.052

Mula, J., Poler, R., García-Sabater, J. \& Lario, F. (2006). Models for production planning under uncertainty: A review. International Journal of Production Economics, 103(1), 271-285. https://doi.org/10.1016/j.ijpe.2005.09.001

Munim, Z. \& Haralambides, H. (2018). Competition and cooperation for intermodal container transhipment: A network optimization approach. Research in Transportation Business \& Management, 26, 87-99. https://doi.org/10.1016/j.rtbm.2018.03.004

Naciones Unidas. (2015). Transforming our world: the 2030 Agenda for Sustainable Development. https://sdgs.un.org/2030agenda

Naciones Unidas. (2020). Marco de indicadores mundiales para los Objetivos de Desarrollo Sostenible y metas de la Agenda 2030 para el Desarrollo Sostenible, E/CN.3/2020/2. https://unstats.un.org/sdgs/indicators/Global\%20Indicator\%20Framework\%20after\%202020\%20review_Spa .pdf

Nickel, S., Saldanha-da-Gama, F. \& Ziegler, H. (2012). A multi-stage stochastic supply network design problem with financial decisions and risk management. Omega, 40(5), 511-524. https://doi.org/10.1016/j.omega.2011.09.006

Nieto, C. (2011). El ferrocarril en Colombia y la búsqueda de un país. Apuntes, 24(1), 62-75.

Nourbakhsh, S. Bai, Y., Maia, G., Ouyang, Y. \& Rodriguez, L. (2016). Grain supply chain network design and logistics planning for reducing post-harvest loss. Biosystems Engineering, 151, 105-115. https://doi.org/10.1016/j.biosystemseng.2016.08.011

Olander, S. (2007). Stakeholder impact analysis in construction project management. Construction Management and Economics, 25(3), 277-287. https://doi.org/10.1080/01446190600879125

Oren, O., Yechieli, Y., Böhlke, J. \& Dody, A. (2004). Contamination of groundwater under cultivated fields in an arid environment, central Arava Valley, Israel. Journal of Hydrology, 290(3-4), 312-328. https://doi.org/10.1016/j.jhydrol.2003.12.016

Ortega, E., Otero, I. \& Mancebo, S. (2014). TITIM GIS-tool: A GIS-based decision support system for measuring the territorial impact of transport infrastructures. Expert Systems with Applications, 41(16), 7641-7652. https://doi.org/10.1016/j.eswa.2014.05.028

Ortiz-Astorquiza, C., Contreras, I. \& Laporte, G. (2018). Multi-level facility location problems. In European Journal of Operational Research (Vol. 267, Issue 3, pp. 791-805). https://doi.org/10.1016/j.ejor.2017.10.019

Ospina, G. (2016). El papel de las vías secundarias y los caminos vecinales en el desarrollo de Colombia. Revista de Ingeniería, 44, 20-27.

Pallme, D., Lambert, B., Miller, C. \& Lipinski, M. (2015). A review of public and private intermodal railroad development in the Memphis region. Research in Transportation Business \& Management, 14, 44-55. https://doi.org/10.1016/j.rtbm.2014.10.011

Papadimitriou, D., Colle, D. \& Demeester, P. (2018). Mixed integer optimization for the combined capacitated facility location-routing problem. Annals of Telecommunications, 73(1-2), 37-62. https://doi.org/10.1007/s12243-017-0620-5

Parques Nacionales Naturales de Colombia. (2021). Portafolio de nuevas áreas y ampliaciones del ámbito de gestión nacional. https://www.parquesnacionales.gov.co/portal/es/sistema-nacional-de-areas-protegidassinap/portafolio-de-nuevas-areas-protegidas-del-sistemas-de-parques-nacionales/

Peña-Mora, F. \& Li, M. (2001). Dynamic planning and control methodology for design/build fast-track construction projects. Journal of Construction Engineering and Management, 127(1), 1-17. https://doi.org/10.1061/(ASCE)0733-9364(2001)127:1(1)

Peng, Y., Li, Z. \& Schonfeld, P. (2019). Development of rail transit network over multiple time periods. Transportation Research Part A: Policy and Practice, 121, 235-250. https://doi.org/10.1016/j.tra.2019.01.017 
Penyalver, D., Turró, M. \& Williamson, J. (2019). Measuring the value for money of transport infrastructure procurement; an intergenerational approach. Transportation Research Part A: Policy and Practice, 119, 238254. https://doi.org/10.1016/j.tra.2018.11.013

Phillips, B., Bullock, J., Osborne, J. \& Gaston, K. (2020). Ecosystem service provision by road verges. Journal of Applied Ecology, 57(3), 488-501. https://doi.org/10.1111/1365-2664.13556

Pinto, J., Mistage, O., Bilotta, P. \& Helmers, E. (2018). Road-rail intermodal freight transport as a strategy for climate change mitigation. Environmental Development, 25, 100-110. https://doi.org/10.1016/j.envdev.2017.07.005

Pita-Pico, R. (2016). Colonización, conflicto y cultura en la región del Magdalena Medio: Entre la diversidad y la estigmatización. Revista Temas, 10, 65-80. https://doi.org/10.15332/rt.v0i10.1606

Pourjavad, E. \& Mayorga, R. (2019). Multi-objective fuzzy programming of closed-loop supply chain considering sustainable measures. International Journal of Fuzzy Systems, 21(2), 655-673. https://doi.org/10.1007/s40815-018-0551-y

Puerto, J., Ricca, F. \& Scozzari, A. (2018). Extensive facility location problems on networks: an updated review. TOP, 26(2), 187-226. https://doi.org/10.1007/s11750-018-0476-5

Puettmann, C. \& Stadtler, H. (2010). A collaborative planning approach for intermodal freight transportation. OR Spectrum, 32(3), 809-830. https://doi.org/10.1007/s00291-010-0211-6

Rad, R. \& Nahavandi, N. (2018). A novel multi-objective optimization model for integrated problem of green closed loop supply chain network design and quantity discount. Journal of Cleaner Production, 196, 15491565. https://doi.org/10.1016/j.jclepro.2018.06.034

Rahmaniani, R. \& Ghaderi, A. (2013). A combined facility location and network design problem with multi-type of capacitated links. Applied Mathematical Modelling, 37(9), 6400-6414. https://doi.org/10.1016/j.apm.2013.01.001

Rahmaniani, R. \& Ghaderi, A. (2015). An algorithm with different exploration mechanisms: Experimental results to capacitated facility location/network design problem. Expert Systems with Applications, 42(7), 3790-3800. https://doi.org/10.1016/j.eswa.2014.12.051

Rahmati, R. \& Neghabi, H. (2021). Adjustable robust balanced hub location problem with uncertain transportation cost. Computational and Applied Mathematics, 40(14). https://doi.org/10.1007/s40314-020-01405-2

Ranaiefar, F. \& Amelia, R. (2011). Freight transportation externalities. In Logistics Operations and Management (pp. 333-358). Elsevier. https://doi.org/10.1016/B978-0-12-385202-1.00016-5

Red de Información y Comunicación del Sector Agropecuario de Colombia AGRONET (20 abril de 2021). Reportes estadísticos. https://www.agronet.gov.co/estadistica/Paginas/home.aspx?cod=4\#

Reis, V., Fabian Meier, J., Pace, G. \& Palacin, R. (2013). Rail and multi-modal transport. Research in Transportation Economics, 41(1), 17-30. https://doi.org/10.1016/j.retrec.2012.10.005

Rohmer, S., Gerdessen, J. \& Claassen, G. (2019). Sustainable supply chain design in the food system with dietary considerations: A multi-objective analysis. European Journal of Operational Research, 273(3), 1149-1164. https://doi.org/10.1016/j.ejor.2018.09.006

Romero, C. \& Rehman, T. (2003). Multiobjective programming. In Multiple Criteria Analysis for Agricultural Decisions (pp. 47-61). https://doi.org/10.1016/S0926-5589(03)80006-7

Rothenbächer, A., Drexl, M. \& Irnich, S. (2016). Branch-and-price-and-cut for a service network design and hub location problem. European Journal of Operational Research, 255(3), 935-947. https://doi.org/10.1016/j.ejor.2016.05.058

Rowsell, J., Brooks, M., Behrens, K., Heaver, T. \& Lawson, J. (2018). Valuing freight transport: A Canadian example of the role of selected methodologies. Research in Transportation Business \& Management, 29, 7784. https://doi.org/10.1016/j.rtbm.2018.06.001

Rožić, T., Rogić, K. \& Bajor, I. (2016). Research trends of inland terminals: A literature review. PROMET Traffic\&Transportation, 28(5), 539-548. https://doi.org/10.7307/ptt.v28i5.2090

Saeed, N. (2013). Cooperation among freight forwarders: Mode choice and intermodal freight transport. Research in Transportation Economics, 42(1), 77-86. https://doi.org/10.1016/j.retrec.2012.11.005

Sahebjamnia, N., Fathollahi-Fard, A. \& Hajiaghaei-Keshteli, M. (2018). Sustainable tire closed-loop supply chain network design: Hybrid metaheuristic algorithms for large-scale networks. Journal of Cleaner Production, 196, 273-296. https://doi.org/10.1016/j.jclepro.2018.05.245

Sanci, E. \& Daskin, M. S. (2019). Integrating location and network restoration decisions in relief networks under uncertainty. European Journal of Operational Research, 279(2), 335-350. https://doi.org/10.1016/j.ejor.2019.06.012 
Santos, B., Limbourg, S. \& Carreira, J. S. (2015). The impact of transport policies on railroad intermodal freight competitiveness - The case of Belgium. Transportation Research Part D: Transport and Environment, 34, 230-244. https://doi.org/10.1016/j.trd.2014.10.015

Santos, M. \& Villatoro, P. (2018). A multidimensional poverty index for latin america. Review of Income and Wealth, 64(1), 52-82. https://doi.org/10.1111/roiw.12275

Schneider, M. \& Drexl, M. (2017). A survey of the standard location-routing problem. Annals of Operations Research, 259(1-2), 389-414. https://doi.org/10.1007/s10479-017-2509-0

Shayanfar, E., Abianeh, A., Schonfeld, P. \& Zhang, L. (2016). Prioritizing interrelated road projects using metaheuristics. Journal of Infrastructure Systems, 22(2), 04016004. https://doi.org/10.1061/(ASCE)IS.1943555X.0000293

Shayanfar, E. \& Schonfeld, P. (2019). Selecting and scheduling interrelated road projects with uncertain demand. Transportmetrica A: Transport Science, 15(2), 1712-1733. https://doi.org/10.1080/23249935.2019.1639083

Sherafati, M., Bashiri, M., Tavakkoli-Moghaddam, R. \& Pishvaee, M. (2019). Supply chain network design considering sustainable development paradigm: A case study in cable industry. Journal of Cleaner Production, 234, 366-380. https://doi.org/10.1016/j.jclepro.2019.06.095

Shishebori, D. \& Jabalameli, M. (2013). A new integrated mathematical model for optimizing facility location and network design policies with facility disruptions. Life Science Journal, 10(1), 1896-1906. https://doi.org/10.1016/j.tine.2019.100122

Shishebori, D., Yousefi-Babadi, A. \& Davoudi, A. (2016). A multi objective FLND problem under uncertain environment using robust optimization: A heuristic approach and case study. 2016 12th International Conference on Industrial Engineering (ICIE), 83-89. https://doi.org/10.1109/INDUSENG.2016.7519353

Shukla, M. \& Jharkharia, S. (2013). Agri-fresh produce supply chain management: a state-of-the-art literature review. International Journal of Operations \& Production Management, 33(2), 114-158. https://doi.org/10.1108/01443571311295608

Silva, A., Aloise, D., Coelho, L. \& Rocha, C. (2021). Heuristics for the dynamic facility location problem with modular capacities. European Journal of Operational Research, 290(2), 435-452. https://doi.org/10.1016/j.ejor.2020.08.018

Silva, J. (21 de septiembre 2012). Serranía de San Lucas, un oasis condenado a morir: Uno de los ecosistemas más ricos del país clama la creación de un parque nacional en la zona. El Tiempo. https://www.eltiempo.com/archivo/documento/CMS-12243772

Sociedad Vías de las Américas. (15 de junio de 2021). Mapas proyecto vía de las Américas. https://www.transversaldelasamericas.com/images/mapas/PLANO_LOCALIZACI\%C3\%93N_PROYECTO _ACTUALIZADO_SEPT_2017.jpg

Stank, T. \& Roath, A. (1998). Some propositions on intermodal transportation and logistics facility development: Shippers' perspectives. Transportation Journal, 13-24. https://www.jstor.org/stable/20713350

SteadieSeifi, M., Dellaert, N., Nuijten, W., Van Woensel, T. \& Raoufi, R. (2014). Multimodal freight transportation planning: A literature review. European Journal of Operational Research, 233(1), 1-15. https://doi.org/10.1016/j.ejor.2013.06.055

Sternberg, H., Hagen, A., Paganelli, P. \& Lumsden, K. (2010). Intelligent cargo - enabling future's sustainable and accountable transportation system. World Journal of Science, Technology and Sustainable Development, 7(3), 253-262. https://doi.org/10.1108/20425945201000016

Sun, O. \& Fan, N. (2020). A Review on optimization methods for biomass supply chain: Models and algorithms, sustainable issues, and challenges and opportunities. Process Integration and Optimization for Sustainability, 4(3), 203-226. https://doi.org/10.1007/s41660-020-00108-9

Szeto, W., Jiang, Y., Wang, D. \& Sumalee, A. (2015). A sustainable road network design problem with land use transportation interaction over time. Networks and Spatial Economics, 15(3), 791-822. https://doi.org/10.1007/s11067-013-9191-9

Taha, H. (2012). Investigación de operaciones (Pearson (Ed.); 11th ed.).

Trebilcock, M. \& Rosenstock, M. (2015). Infrastructure public-private partnerships in the developing world: lessons from recent experience. The Journal of Development Studies, 51(4), 335-354. https://doi.org/10.1080/00220388.2014.959935

Tripathi, S., Hudnurkar, M. \& Ambekar, S. (2021). Selection of mode of transport for freight movement. International Journal of Innovation Science, 13(3), 268-285. https://doi.org/10.1108/IJIS-09-2020-0173

Tsao, Y. \& Thanh, V. (2019). A multi-objective mixed robust possibilistic flexible programming approach for sustainable seaport-dry port network design under an uncertain environment. Transportation Research Part 
E: Logistics and Transportation Review, 124, 13-39. https://doi.org/10.1016/j.tre.2019.02.006

Tudorica, A. \& Banacu, C. (2017). The potential of intermodal transport projects in Romania. Proceedings of the International Conference on Business Excellence, 11(1), 649-659. https://doi.org/10.1515/picbe-2017-0069

Ukkusuri, S. \& Patil, G. (2009). Multi-period transportation network design under demand uncertainty. Transportation Research Part B: Methodological, 43(6), 625-642. https://doi.org/10.1016/j.trb.2009.01.004

UNECE. (2001). Terminology on Combined Transport. Geneva: United Nations.

Van Zyl, F. (1992). Freight transport in a changing environment: Implementation of deregulation. International Journal of Physical Distribution \& Logistics Management, 22(2), 33-39. https://doi.org/10.1108/EUM0000000000406

Verweij, K. (2011). Synchronic modalities: Critical success factors. In: P. J. van der Sterre (Ed.), Logistics yearbook edition 2011 (pp. 75-92).

Wątróbski, J., Jankowski, J., Ziemba, P., Karczmarczyk, A. \& Zioło, M. (2019). Generalised framework for multicriteria method selection. Omega, 86, 107-124. https://doi.org/10.1016/j.omega.2018.07.004

Wei, W., Wu, D., Wu, Q., Shafie-Khah, M. \& Catalao, J. (2019). Interdependence between transportation system and power distribution system: a comprehensive review on models and applications. Journal of Modern Power Systems and Clean Energy, 7(3), 433-448. https://doi.org/10.1007/s40565-019-0516-7

Wiegmans, B. \& Behdani, B. (2018). A review and analysis of the investment in, and cost structure of, intermodal rail terminals. Transport Reviews, 38(1), 33-51. https://doi.org/10.1080/01441647.2017.1297867

Wieslaw, K., Tadeusz, N. \& Michal, S. (2016). Innovative project of prototype railway wagon and intermodal transport system. In L. Rafalski \& A. Zofka (Eds.), Transport Research Arena Tra2016 (pp. 615-624). https://doi.org/10.1016/j.trpro.2016.05.307

Wiley, J. \& Timmermans, H. (2009). Modelling portfolio choice in transportation research. Transport Reviews, 29(5), 569-586. https://doi.org/10.1080/01441640902829462

Wilmsmeier, G., Monios, J. \& Rodrigue, J. (2015). Drivers for outside-in port hinterland integration in Latin America: The case of Veracruz, Mexico. Research in Transportation Business \& Management, 14, 34-43. https://doi.org/10.1016/j.rtbm.2014.10.013

Wisner, J. (1999). A study of successful quality improvement programs in the transportation industry. Benchmarking: An International Journal, 6(2), 147-163. https://doi.org/10.1108/14635779910269759

Witte, P., Van Oort, F., Wiegmans, B. \& Spit, T. (2014). European corridors as carriers of dynamic agglomeration externalities? $\quad$ European $\quad$ Planning $\quad$ Studies, $22(11), \quad 2350$. https://doi.org/10.1080/09654313.2013.837153

Woxenius, J. (2007). Generic framework for transport network designs: Applications and treatment in intermodal freight transport literature. Transport Reviews, 27(6), 733-749. https://doi.org/10.1080/01441640701358796

Xiang, Y., Yang, W., Liu, J. \& Li, F. (2016). Multi-objective distribution network expansion incorporating electric vehicle charging stations. Energies, 9(11), 909. https://doi.org/10.3390/en9110909

Xiao, Y., Norris, C., Lenzen, M., Norris, G. \& Murray, J. (2017). How social footprints of nations can assist in achieving the sustainable development goals. Ecological Economics, 135, 55-65. https://doi.org/10.1016/j.ecolecon.2016.12.003

Xie, F., Huang, Y. \& Eksioglu, S. (2014). Integrating multimodal transport into cellulosic biofuel supply chain design under feedstock seasonality with a case study based on California. Bioresource Technology, 152, 1523. https://doi.org/10.1016/j.biortech.2013.10.074

$\mathrm{Xu}, \mathrm{X}$., Chen, A. \& Yang, C. (2016). A review of sustainable network design for road networks. KSCE Journal of Civil Engineering, 20(3), 1084-1098. https://doi.org/10.1007/s12205-016-1729-1

Yannis, G., Kopsacheili, A., Dragomanovits, A. \& Petraki, V. (2020). State-of-the-art review on multi-criteria decision-making in the transport sector. Journal of Traffic and Transportation Engineering (English Edition), 7(4), 413-431. https://doi.org/10.1016/j.jtte.2020.05.005

Yoon, M. \& Current, J. (2008). The hub location and network design problem with fixed and variable arc costs: formulation and dual-based solution heuristic. Journal of the Operational Research Society, 59(1), 80-89. https://doi.org/10.1057/palgrave.jors.2602307

Zhang, M, Janic, M. \& Tavasszy, L. (2015). A freight transport optimization model for integrated network, service, and policy design. Transportation Research Part E: Logistics and Transportation Review, 77, 61-76. https://doi.org/10.1016/j.tre.2015.02.013

Zhang, Mo, Wiegmans, B. \& Tavasszy, L. (2013). Optimization of multimodal networks including environmental costs: A model and findings for transport policy. Computers in Industry, 64(2), 136-145. 
https://doi.org/10.1016/j.compind.2012.11.008

Zhang, X. \& Waller, S. (2018). Mixed-vehicular aggregated transportation network design considering en-route recharge service provision for electric vehicles. Journal of Systems Science and Complexity, 31(5), 13291349. https://doi.org/10.1007/s11424-018-7165-1

Zhao, X., Ke, Y., Zuo, J., Xiong, W. \& Wu, P. (2020). Evaluation of sustainable transport research in 2000-2019. Journal of Cleaner Production, 256, 120404. https://doi.org/10.1016/j.jclepro.2020.120404

Zhou, B., Li, Z., Patel, H., Roshandeh, A. \& Wang, Y. (2014). Risk-based two-step optimization model for highway transportation investment decision-making. Journal of Transportation Engineering, 140(5), 04014007. https://doi.org/10.1061/(ASCE)TE.1943-5436.0000652

Zhou, M., Duan, Y., Yang, W., Pan, Y. \& Zhou, M. (2018). Capacitated multi-modal network flow models for minimizing total operational cost and $\mathrm{CO}^{2}$ emission. Computers \& Industrial Engineering, 126, 361-377. https://doi.org/10.1016/j.cie.2018.09.049 


\section{Anexos}

Anexo A. Datos de área sembrada y producción por alternativa agrícola, municipio y año.

\begin{tabular}{|c|c|c|c|c|c|c|c|c|c|c|c|c|c|}
\hline \multirow{2}{*}{\multicolumn{2}{|c|}{ Producto }} & \multicolumn{6}{|c|}{ Área Sembrada (hectáreas)* } & \multicolumn{6}{|c|}{ Producción (toneladas) ${ }^{* *}$} \\
\hline & & M1 & M2 & M3 & M4 & M5 & M6 & M1 & M2 & M3 & M4 & M5 & M6 \\
\hline \multirow{16}{*}{ 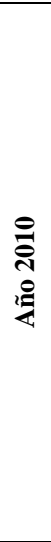 } & P1 & & & & & & & & & & & & \\
\hline & $\mathrm{P} 2$ & & & 259 & & & & & & 1440 & & & \\
\hline & P3 & & & & & & & & & & & & \\
\hline & P4 & & & & 650 & & 820 & & & & 1200 & & 1386 \\
\hline & P5 & & & & & & & & & & & & \\
\hline & P6 & 87 & 574 & 117 & 1160 & 2478 & 744 & 18 & 62 & 19 & 120 & 294 & 126 \\
\hline & P7 & & & & & 1030 & & & & & & 510 & \\
\hline & P8 & & & & & 360 & & & & & & 1960 & \\
\hline & P9 & & 6 & & 297 & & 331 & & & & & & \\
\hline & P10 & & & 950 & & 1090 & 180 & & & 400 & & 538 & 41 \\
\hline & P11 & 1750 & 1320 & 2280 & 1765 & 1100 & 2700 & 786 & 1932 & 2882 & 1514 & 1510 & 2220 \\
\hline & P12 & 122 & 729 & 563 & 4442 & & 4949 & & 2273 & & 14375 & & 14056 \\
\hline & P13 & & & & & & & & & & & & \\
\hline & P14 & & & & & 30 & & & & & & 30 & \\
\hline & P15 & & 100 & & & 400 & & & 500 & & & 1750 & \\
\hline & $\mathrm{P} 16$ & 180 & 850 & 750 & 850 & 700 & 1200 & 750 & 4242 & 2795 & 6400 & 3250 & 4800 \\
\hline & & & & & & & & & & & & & \\
\hline \multirow{16}{*}{ 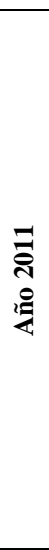 } & P1 & & & & & & & & & & & & \\
\hline & P2 & 3 & & 257 & & & & & & 379 & & & \\
\hline & P3 & & & & & & & & & & & & \\
\hline & P4 & & & 950 & 800 & & 700 & & & 1520 & 1225 & & 1380 \\
\hline & P5 & & & & & & & & & & & & \\
\hline & P6 & 87 & 574 & 117 & 1160 & 2478 & 744 & 18 & 105 & 19 & 120 & 294 & 126 \\
\hline & P7 & & & & & 1030 & & & & & & 510 & \\
\hline & P8 & & & & & 360 & & & & & & 2142 & \\
\hline & P9 & & 6 & & 297 & & 331 & & 6 & & & & \\
\hline & P10 & & & 1600 & & 880 & 215 & & & 549 & & 216 & 70 \\
\hline & P11 & 650 & 800 & 1950 & 960 & 1200 & 3400 & 2130 & 810 & 3984 & 2031 & 1360 & 2545 \\
\hline & $\mathrm{P} 12$ & 122 & 1443 & 1950 & 4442 & & 4949 & & 2293 & & 14440 & & 13962 \\
\hline & P13 & & & & & & & & & & & & \\
\hline & P14 & & & & & 30 & & & & & & 30 & \\
\hline & P15 & & 100 & & & 400 & & & 500 & & & 1750 & \\
\hline & P16 & 200 & 360 & 800 & 600 & 600 & 400 & 600 & 1320 & 3500 & 4640 & 2800 & 2100 \\
\hline & & & & & & & & & & & & & \\
\hline \multirow{16}{*}{ 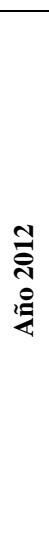 } & P1 & & & & & 115 & & & & & & 80 & \\
\hline & P2 & 3 & & 249 & & & & 5 & & 257 & & & \\
\hline & P3 & & & 450 & & & & & & & & & \\
\hline & P4 & 120 & & 800 & 280 & & 770 & 1 & & 2798 & 750 & & 1518 \\
\hline & P5 & & & & & & & & & & & & \\
\hline & P6 & 70 & 670 & 137 & 1340 & 3040 & 920 & 14 & 65 & 44 & 390 & 1150 & 290 \\
\hline & P7 & & & & & 1390 & & & & & & 653 & \\
\hline & P8 & & & & & 470 & & & & & & 3000 & \\
\hline & P9 & & 9 & & 373 & & 333 & & 6 & & & & \\
\hline & P10 & & & 1610 & & 870 & 200 & & & 800 & & 430 & 93 \\
\hline & P11 & 1600 & 650 & 4100 & 820 & 1250 & 3650 & 1530 & 720 & 3165 & & 1629 & 4490 \\
\hline & P12 & 140 & 1283 & 690 & 6750 & & 5600 & 360 & 3171 & 960 & 18126 & & 16450 \\
\hline & P13 & & & & & & & & & & & & \\
\hline & P14 & & & & & 25 & & & & & & 100 & \\
\hline & P15 & & & & & 505 & & & & & & 2760 & \\
\hline & P16 & 180 & 450 & 700 & 700 & 650 & 450 & 900 & 4500 & 5600 & 5440 & 3900 & 3010 \\
\hline & & & & & & & & & & & & & \\
\hline \multirow{10}{*}{ 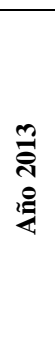 } & P1 & & & & & 120 & & & & & & 100 & \\
\hline & P2 & & & 403 & & & & 5 & & 324 & & & \\
\hline & P3 & & & 280 & & & & & & 2145 & & & \\
\hline & P4 & 150 & & 400 & 900 & & 843 & 360 & & 1200 & 2940 & & 2180 \\
\hline & P5 & & & & & & & & & & & & \\
\hline & P6 & 70 & 710 & 137 & 1400 & 3200 & 1005 & 18 & 81 & 36 & 372 & 960 & 262 \\
\hline & P7 & & & 77 & & 922 & & & & 41 & & 355 & \\
\hline & P8 & & & & & 500 & & & & & & 3760 & \\
\hline & P9 & & 9 & & 200 & & 223 & & & & & & \\
\hline & P10 & & & 1300 & & 1100 & 255 & & & 685 & & 815 & 90 \\
\hline
\end{tabular}




\begin{tabular}{|c|c|c|c|c|c|c|c|c|c|c|c|c|c|}
\hline & P11 & 2300 & 800 & 3550 & 2350 & 2460 & 4200 & 3519 & 1400 & 5560 & 3720 & 4675 & 4330 \\
\hline & P12 & 140 & 883 & 979 & 7200 & & 6100 & 396 & 3444 & 1798 & 18092 & & 16830 \\
\hline & P13 & & & & & & & & & & & & \\
\hline & P14 & & & & & 25 & & & & & & 100 & \\
\hline & P15 & & & & & 550 & & & & & & 3000 & \\
\hline & P16 & 170 & 500 & 850 & 1200 & 600 & 560 & 1190 & 4000 & 6000 & 9600 & 3600 & 3920 \\
\hline \multirow{16}{*}{ 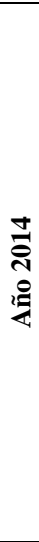 } & P1 & & & & & 120 & & & & & & 200 & \\
\hline & $\mathrm{P} 2$ & & & 388 & & & & & & 645 & & & \\
\hline & P3 & & & 213 & & & & & & 994 & & & \\
\hline & $\mathrm{P} 4$ & 150 & & & 900 & & 840 & 450 & & & 3000 & & 2460 \\
\hline & P5 & & & & & & & & & & & & \\
\hline & P6 & 70 & 710 & 137 & 1400 & 3290 & 1005 & 21 & 54 & 18 & 372 & 1345 & 262 \\
\hline & P7 & & & 77 & & 1040 & & & & 45 & & 562 & \\
\hline & P8 & & & & & 560 & & & & & & 3840 & \\
\hline & P9 & & 9 & & 200 & & 223 & & & & 27 & & 63 \\
\hline & P10 & & & 1370 & & 1250 & 260 & & & 737 & & 505 & 150 \\
\hline & P11 & 2300 & 500 & 3550 & 2350 & 2506 & 4200 & 4300 & 1600 & 6600 & 4420 & 4920 & 6041 \\
\hline & P12 & 140 & 883 & 979 & 7200 & & 6100 & 415 & 3444 & 1800 & 20631 & & 17850 \\
\hline & P13 & & & & & & & & & & & & \\
\hline & P14 & & & & & 25 & & & & & & 100 & \\
\hline & P15 & & & & & 539 & & & & & & 3006 & \\
\hline & P16 & 170 & 500 & 850 & 1200 & 900 & 560 & 1190 & 4000 & 7650 & 9600 & 5400 & 3920 \\
\hline & & & & & & & & & & & & & \\
\hline \multirow{16}{*}{ 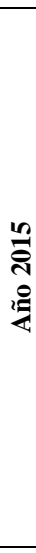 } & $\mathrm{P} 1$ & & & & & 123 & & & & & & 215 & \\
\hline & $\mathrm{P} 2$ & & & 350 & & & & & & 620 & & & \\
\hline & $\mathrm{P} 3$ & & & 255 & & & & & & 1004 & & & \\
\hline & $\mathrm{P} 4$ & 150 & & & 750 & & 860 & 450 & & & 2025 & & 2280 \\
\hline & P5 & & & & & & & & & & & & \\
\hline & P6 & 70 & 710 & 137 & 1400 & 3290 & 1005 & 18 & 120 & 31 & 372 & 1431 & 262 \\
\hline & P7 & & & 81 & & 1078 & & & & 65 & & 1025 & \\
\hline & P8 & & & & & 565 & & & & & & 3840 & \\
\hline & P9 & & 9 & & 200 & & 223 & & & & & & \\
\hline & P10 & & & 1350 & & 1150 & 280 & & & 775 & & 612 & 129 \\
\hline & $\mathrm{P} 11$ & 1450 & 490 & 4500 & 2100 & 1500 & 3600 & 2800 & 900 & 8200 & 3400 & 2412 & 6100 \\
\hline & P12 & 140 & 863 & 979 & 7200 & & 6100 & 327 & 2564 & 1780 & 15712 & & 15147 \\
\hline & P13 & & & & & & & & & & & & \\
\hline & P14 & & & & & 25 & & & & & & 100 & \\
\hline & P15 & & & & & 710 & & & & & & 4080 & \\
\hline & P16 & 180 & 550 & 900 & 1100 & 950 & 580 & 770 & 3600 & 6400 & 7000 & 4800 & 3500 \\
\hline & & & & & & & & & & & & & \\
\hline \multirow{16}{*}{ 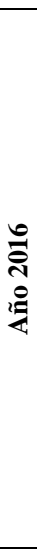 } & P1 & & & & & 123 & & & & & & 344 & \\
\hline & $\mathrm{P} 2$ & & & 381 & & & & & & 413 & & & \\
\hline & P3 & & & 1169 & 172 & & & & & 3106 & 300 & & \\
\hline & $\mathrm{P} 4$ & 24 & & & 250 & & 860 & 306 & & & 750 & & 2100 \\
\hline & P5 & 59 & 87 & & 58 & & 30 & 165 & 145 & & 174 & & 80 \\
\hline & P6 & 120 & 767 & 140 & 1350 & 3490 & 1005 & 48 & 140 & 32 & 314 & 1272 & 262 \\
\hline & P7 & & & 81 & & 1102 & & & & 66 & & 1063 & \\
\hline & P8 & & & & & 585 & & & & & & 4520 & \\
\hline & P9 & & 9 & & 370 & & 220 & & & & 153 & & \\
\hline & P10 & & & 1452 & & 270 & 280 & & & 752 & & 354 & 114 \\
\hline & P11 & 3500 & 510 & 4565 & 1650 & 1700 & 3600 & 2880 & 729 & 7290 & 2550 & 2700 & 4700 \\
\hline & P12 & 140 & 915 & 1038 & 11950 & & 6100 & 280 & 2324 & 1613 & 13437 & & 12954 \\
\hline & P13 & & & & 35 & & & & & & 150 & & \\
\hline & P14 & & & & & 25 & & & & & & 75 & \\
\hline & P15 & 250 & & & 950 & 810 & & 1000 & & & 11400 & 4615 & \\
\hline & P16 & 900 & 545 & 873 & 850 & 1000 & 580 & 900 & 3529 & 6022 & 12750 & 6000 & 3500 \\
\hline & & & & & & & & & & & & & \\
\hline \multirow{13}{*}{ 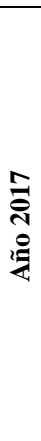 } & $\mathrm{P} 1$ & & & & & 123 & & & & & & 1230 & \\
\hline & $\mathrm{P} 2$ & & & 23 & & & & & & 492 & & & \\
\hline & P3 & & & 480 & & & & & & 3628 & 75 & & \\
\hline & $\mathrm{P} 4$ & 50 & & & 1250 & & 980 & 48 & & & 1755 & & 2700 \\
\hline & P5 & 28 & 40 & & 430 & & 10 & 132 & 113 & & 1008 & & 31 \\
\hline & P6 & 60 & 800 & 137 & 1350 & 3350 & 1005 & 24 & 215 & 32 & 314 & 1368 & 302 \\
\hline & P7 & & & 81 & & 1151 & & & & 51 & & 699 & \\
\hline & P8 & & & 8 & & 585 & & & & 40 & & 4680 & \\
\hline & P9 & & 9 & & 370 & & 220 & & & & 51 & & \\
\hline & P10 & & & 600 & & 400 & 330 & & & 589 & & 210 & 185 \\
\hline & P11 & 1000 & 750 & 2300 & 1750 & 1550 & 3500 & 1950 & 991 & 5544 & 2400 & 2700 & 3080 \\
\hline & P12 & 140 & 400 & 979 & 11950 & & 6175 & 373 & 960 & 1724 & 40511 & & 17025 \\
\hline & P13 & & & & 40 & & & & & & 600 & & \\
\hline
\end{tabular}




\begin{tabular}{|c|c|c|c|c|c|c|c|c|c|c|c|c|c|}
\hline & P14 & & & & & 25 & & & & & & 75 & \\
\hline & P15 & 300 & & 200 & 950 & 810 & & 1200 & & 1000 & 9500 & 5670 & \\
\hline & P16 & 30 & 650 & 950 & 900 & 1000 & 600 & 300 & 6000 & 10800 & 11200 & 8100 & 4125 \\
\hline \multirow{17}{*}{ 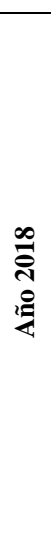 } & & & & & & & & & & & & & \\
\hline & P1 & & & & & 123 & & & & & & 1230 & \\
\hline & P2 & & & & & & & & & & & & \\
\hline & P3 & & & 296 & & & & & & 1479 & & & \\
\hline & P4 & 25 & & & 600 & & 400 & 38 & & & 1200 & & 1320 \\
\hline & P5 & & & & 148 & & & & & & 665 & & \\
\hline & P6 & 60 & 800 & 197 & 1350 & 3350 & 1005 & 24 & 215 & 81 & 314 & 1368 & 302 \\
\hline & P7 & & & 81 & & 1188 & & & & 51 & & 685 & \\
\hline & P8 & & & 8 & & 585 & & & & 48 & & 3510 & \\
\hline & P9 & & 9 & & 370 & & 220 & & & & 85 & & 20 \\
\hline & P10 & & & 600 & & 200 & 200 & & & 420 & & 140 & 100 \\
\hline & P11 & 420 & 400 & 2000 & 900 & 1000 & 2000 & 630 & 620 & 5000 & 1350 & 2000 & 4000 \\
\hline & P12 & 140 & 400 & 979 & 11950 & & 6175 & 373 & 1056 & 2034 & 40511 & & 19239 \\
\hline & P13 & & & & 25 & & & & & & 350 & & \\
\hline & P14 & & & & & 25 & & & & & & 75 & \\
\hline & P15 & 300 & & 200 & 950 & 810 & & 1200 & & 1200 & 11875 & 5670 & \\
\hline & P16 & 50 & 650 & 1000 & 900 & 1300 & 602 & 500 & 6500 & 12000 & 12600 & 11700 & 4559 \\
\hline
\end{tabular}

*M1: Arenal, M2: Cantagallo, M3: Morales, M4: San Pablo, M5: Santa Rosa del Sur, M6: Simití.

**P1: Aguacate, P2: Algodón, P3: Arroz Riego, P4: Arroz Secano Manual, P5: Arroz Secano Mecanizado, P6: Cacao, P7: Café, P8: Caña panelera, P9: Caucho, P10: Frijol, P11: Maíz Tradicional, P12: Palma Aceite, P13: Patilla, P14: Pitahaya, P15: Plátano, P16: Yuca 


\section{Anexo B. Criterios de optimización económico, social y medioambiental identificados en el proceso de revisión de la literatura.}

\begin{tabular}{|c|c|c|c|c|c|}
\hline \multicolumn{2}{|r|}{ Criterios Económicos } & 36 & $\begin{array}{l}\text { Calidad de los } \\
\text { proveedores/productores clave }\end{array}$ & 70 & S-LCA \\
\hline \multicolumn{2}{|c|}{ Costes } & 37 & $\begin{array}{l}\text { Impuestos por localización de la } \\
\text { instalación }\end{array}$ & \multirow{2}{*}{\multicolumn{2}{|c|}{ Criterios Medioambientales }} \\
\hline 1 & $\begin{array}{l}\text { Construcción/apertura de las } \\
\text { instalaciones }\end{array}$ & 38 & Crecimiento de la industria & & \\
\hline 2 & Cierre de instalaciones & 39 & Competitividad en la región anfitriona & \multicolumn{2}{|c|}{ Aire (emisiones) } \\
\hline 3 & $\begin{array}{l}\text { Expansión de la capacidad en las } \\
\text { instalaciones }\end{array}$ & 40 & Valor condicional por riesgo en costes & 71 & $\begin{array}{l}\text { Construcción/operación en la } \\
\text { instalación }\end{array}$ \\
\hline 4 & Expansión de la capacidad de los arcos & 41 & Riesgos en el área de localización & 72 & Producción de materias primas \\
\hline 5 & $\begin{array}{l}\text { Penalización por capacidad inactiva de } \\
\text { la instalación }\end{array}$ & 42 & Puntaje de eficiencia & 73 & Fabricación \\
\hline 6 & Mantenimiento de las instalaciones & 43 & Resiliencia de la cadena de suministro & 74 & $\begin{array}{l}\text { Operación/manipulación en la } \\
\text { instalación }\end{array}$ \\
\hline 7 & Depreciación de las instalaciones & & & 75 & Almacenamiento/inventario \\
\hline 8 & Operación en las instalaciones & \multirow{2}{*}{\multicolumn{2}{|c|}{$\begin{array}{l}\text { Criterios Sociales } \\
\text { Condiciones laborales } \\
\end{array}$}} & 76 & Transporte \\
\hline 9 & Manipulación/preparación de pedidos & & & 77 & $\begin{array}{l}\text { Reprocesamiento del producto } \\
\text { devuelto }\end{array}$ \\
\hline 10 & Compras/adquisiciones & 44 & $\begin{array}{l}\text { Trabajo creado en la } \\
\text { localización/construcción de las } \\
\text { instalaciones }\end{array}$ & 78 & Emisiones capturadas \\
\hline 11 & Producción/fabricación & 45 & $\begin{array}{l}\text { Trabajo creado a lo largo de la cadena } \\
\text { de suministro }\end{array}$ & \multicolumn{2}{|c|}{ Agua } \\
\hline 12 & Subcontratación para la producción & 46 & $\begin{array}{l}\text { Trabajo creado en la actividad de } \\
\text { transporte }\end{array}$ & 79 & Consumida en distintos procesos \\
\hline 13 & $\begin{array}{l}\text { Subcontratación para el } \\
\text { almacenamiento }\end{array}$ & 47 & $\begin{array}{l}\text { Trabajo destruido por el cierre de las } \\
\text { instalaciones }\end{array}$ & 80 & Contaminada por distintos procesos \\
\hline 14 & Inventario & 48 & $\begin{array}{l}\text { Trabajo destruido por el despido de } \\
\text { empleados }\end{array}$ & 81 & Calidad/disponibilidad \\
\hline 15 & $\begin{array}{l}\text { Recuperación de producto o materias } \\
\text { primas }\end{array}$ & 49 & Horas de trabajo & \multicolumn{2}{|c|}{ Suelo } \\
\hline 16 & Reprocesamiento producto devuelto & 50 & Estabilidad laboral & 82 & Para construcción de la instalación \\
\hline 17 & Eliminación de desechos & 51 & $\begin{array}{l}\text { Seguridad/daños en el desempeño } \\
\text { laboral }\end{array}$ & 83 & Para la producción de alimentos \\
\hline 18 & Transporte & 52 & Salud de los empleados & 84 & Contaminación \\
\hline 19 & Enrutamiento & 53 & Satisfacción de los empleados & \multicolumn{2}{|c|}{ Biodiversidad } \\
\hline 20 & Interrupciones en la red & 54 & Lesiones y muertes de los empleados & 85 & Protección \\
\hline 21 & Laborales & \multicolumn{2}{|c|}{ Sociedad } & 86 & Reforestación \\
\hline 22 & Laborales por inactividad & 55 & $\begin{array}{l}\text { Disponibilidad de instalaciones } \\
\text { médicas }\end{array}$ & \multicolumn{2}{|c|}{ Energía } \\
\hline 23 & Selección de proveedores & 56 & $\begin{array}{l}\text { Disponibilidad de instalaciones } \\
\text { educativas }\end{array}$ & 87 & Consumida en distintos procesos \\
\hline 24 & Energía & 57 & Pobreza & 88 & Desaprovechada en distintos procesos \\
\hline 25 & Emisiones & 58 & Población desempleada e inmigrante & \multicolumn{2}{|c|}{ Residuos de material } \\
\hline 26 & $\begin{array}{l}\text { Renovación/reparación } \\
\text { medioambiental }\end{array}$ & 59 & Satisfacción del cliente & 89 & $\begin{array}{l}\text { Generado en la } \\
\text { fabricación/construcción }\end{array}$ \\
\hline 27 & $\begin{array}{l}\text { Penalización por incumplimiento en el } \\
\text { tiempo de servicio }\end{array}$ & \multicolumn{2}{|c|}{ Características del área } & 90 & Tratamiento de desperdicios \\
\hline \multicolumn{2}{|c|}{ Ingresos } & 60 & Estabilidad política & 91 & Disposición de desechos \\
\hline 28 & Por venta del producto & 61 & Barreras comerciales & 92 & $\begin{array}{l}\text { Desperdicio de producto por manejo } \\
\text { inadecuado }\end{array}$ \\
\hline 29 & Por ventas de emisiones & 62 & Tecnología disponible & \multicolumn{2}{|c|}{ Miscelánea con enfoque medioambiental } \\
\hline 30 & Por materiales reciclados & 63 & Nivel de seguridad & 93 & Contaminación acústica \\
\hline 31 & Presupuesto remanente & 64 & Accesibilidad física & 94 & LCA \\
\hline Tier & & 65 & Congestión de tráfico & 95 & Ecoindicator-99 \\
\hline 32 & De espera & 66 & $\begin{array}{l}\text { Nivel de riesgo por localización } \\
\text { potencial }\end{array}$ & 96 & IPCC \\
\hline 33 & De permanencia & 67 & Desarrollo regional & 97 & ReCiPe \\
\hline 34 & De servicio & \multicolumn{2}{|c|}{ Miscelánea con enfoque social } & 98 & Carga de morbilidad ambiental \\
\hline \multicolumn{2}{|c|}{ Miscelánea enfoque económico } & 68 & Responsabilidad social & & \\
\hline 35 & Proximidad a proveedores clave & 69 & Imagen empresarial & & \\
\hline
\end{tabular}


Anexo C. Entradas no nulas de la Matriz A construida para aplicar el esquema de resolución $\mathbf{R}+$ Gurobi en los modelos de programación mono-objetivo y multi-objetivo.

Variables: 1 a 485

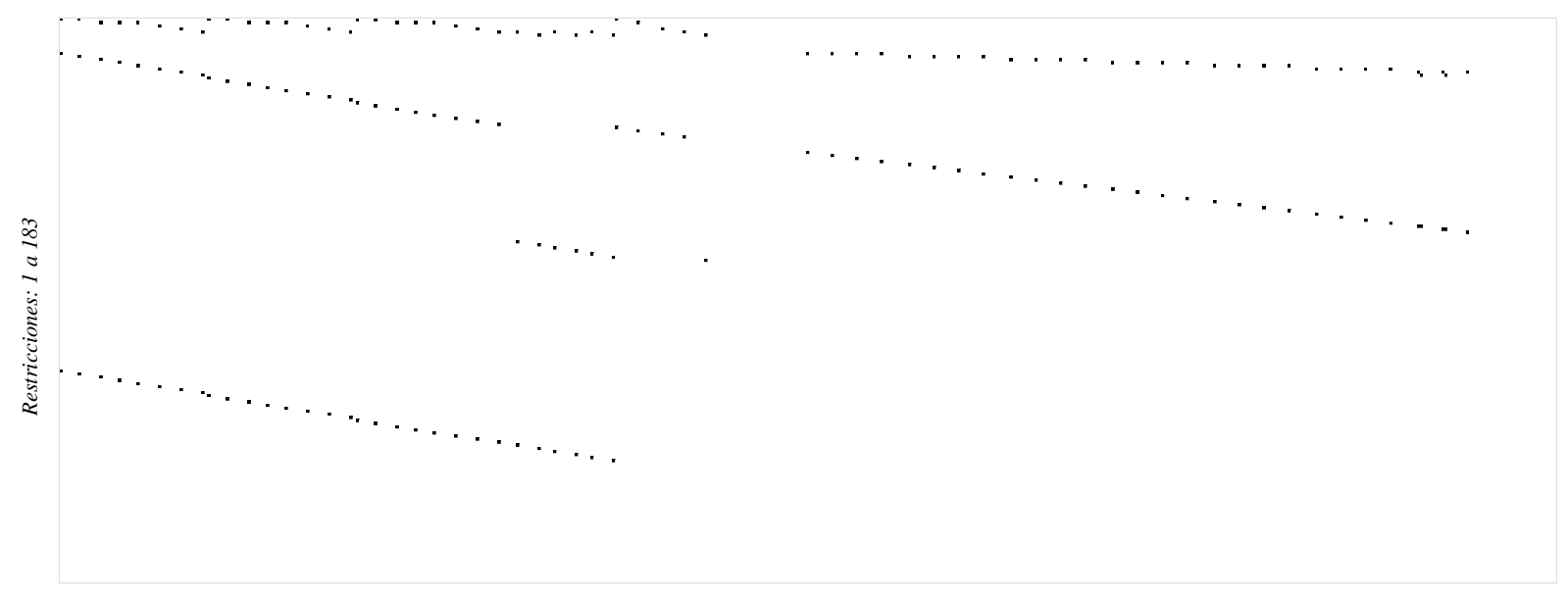

Variables: 486 a 970

Variables: 971 a 1455

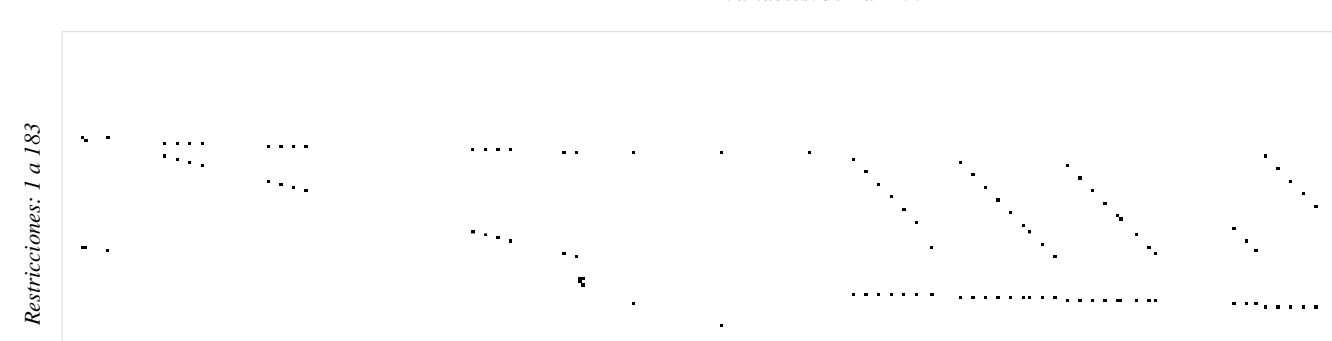


Variables: 1456 a 1940

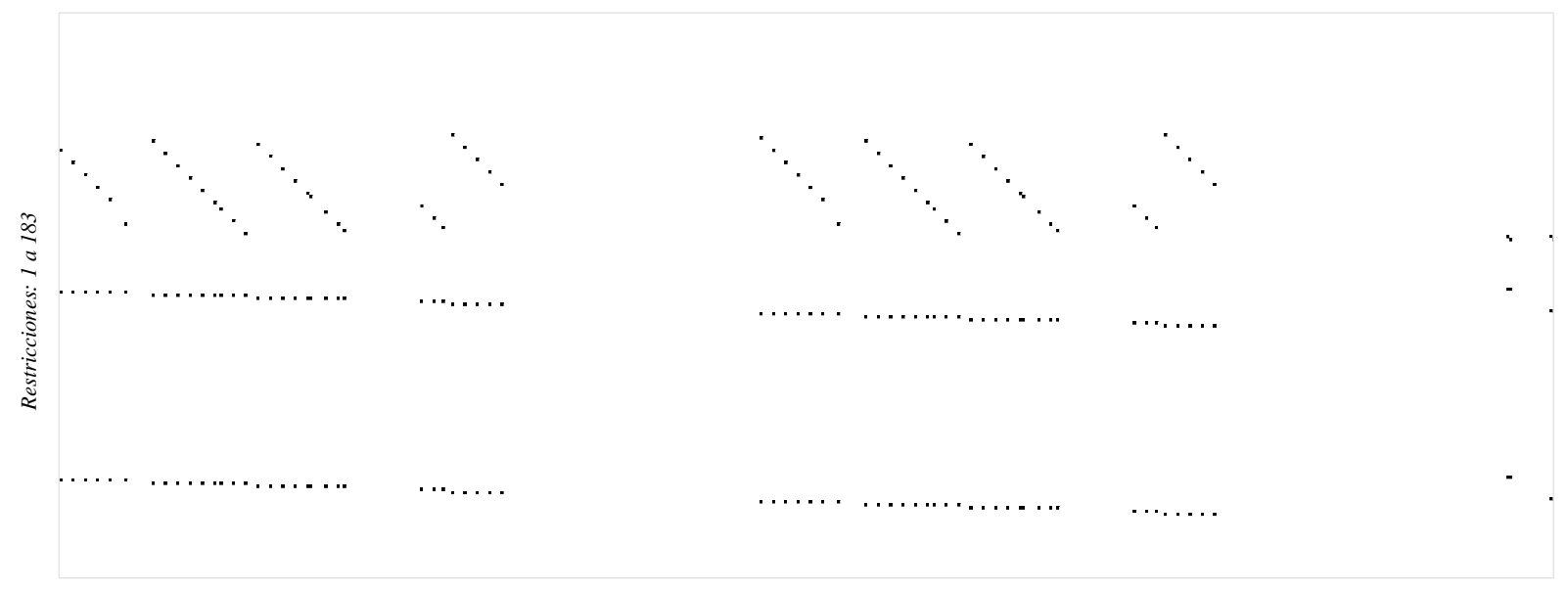

Variables: 1941 a 2426

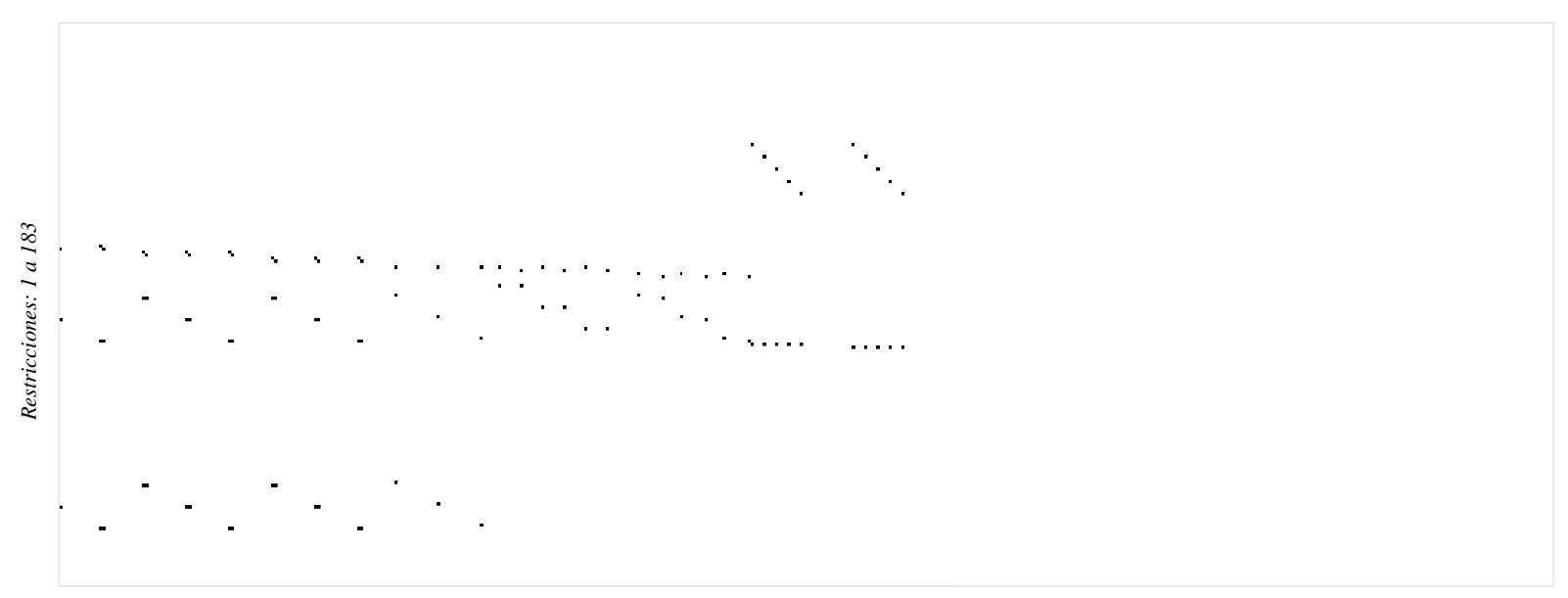

Variables: 2427 a 2912

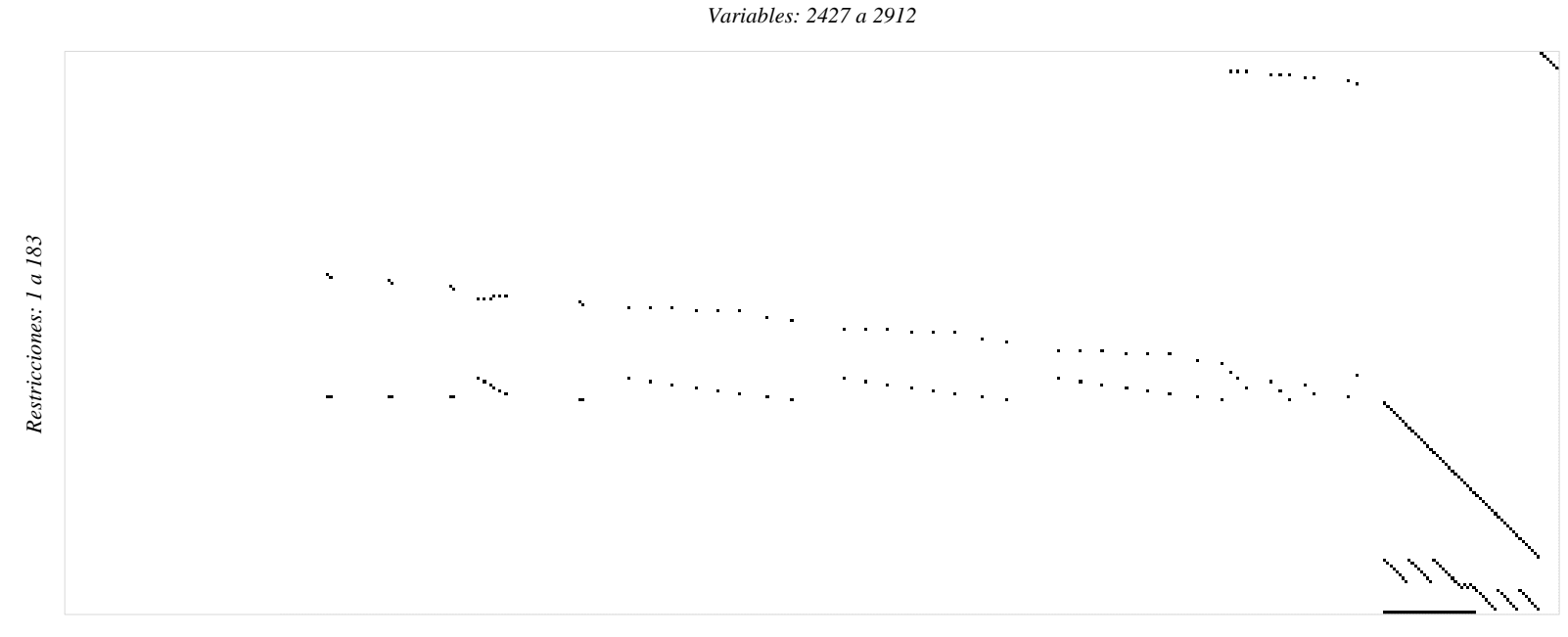




\section{Anexo D. Consolidado del proceso de revisión llevado a cabo en la RSL.}

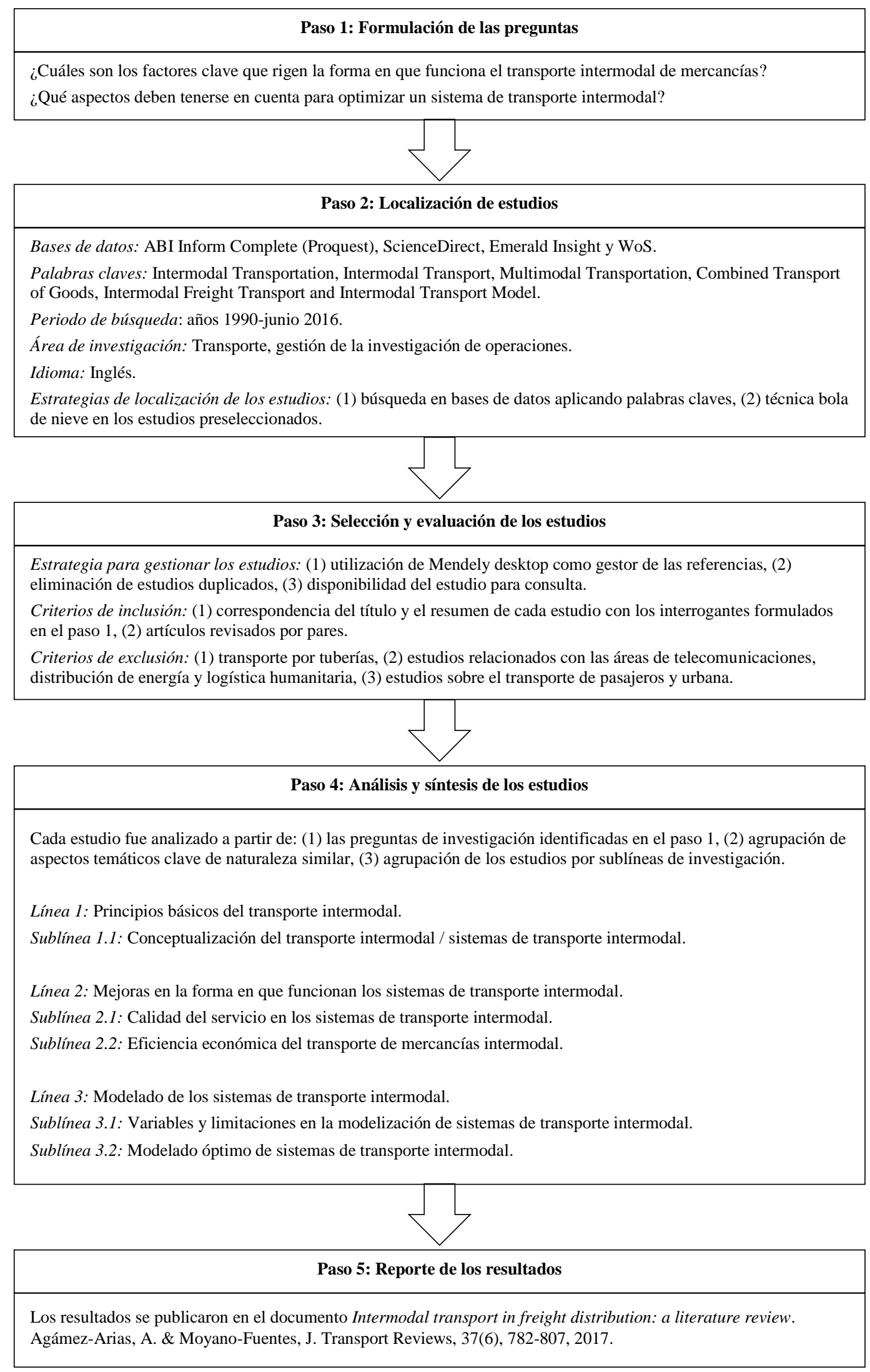




\section{Anexo E. Listado de estudios preseleccionados para la RSL-2EV que se excluyeron en el último filtro.}

\begin{tabular}{|c|c|c|}
\hline Título & Autores & Año \\
\hline An integrated model of facility location and transportation network design & Melkote, S. \& Daskin, M. & 2001 \\
\hline Capacitated facility location/network design problems & Melkote, S. \& Daskin, M. & 2001 \\
\hline Network design: selection and design of links and facility location & Drezner, Z. \& Wesolowsky, G. & 2003 \\
\hline Approximation algorithms for problems combining facility location and network design & Ravi, R. \& Sinha, A. & 2006 \\
\hline Multi-period transportation network design under demand uncertainty & Ukkusuri, S. \& Patil, G. & 2009 \\
\hline Integrated modeling of urban hierarchy and transportation network planning & $\begin{array}{l}\text { Bigotte, J., Krass, D., Antunes, } \\
\text { A. \& Berman, O. }\end{array}$ & 2010 \\
\hline A hybrid model for multi-objective capacitated facility location network design problem & $\begin{array}{l}\text { Jabalameli, M. \& Mortezaei, } \\
\text { M. }\end{array}$ & 2011 \\
\hline $\begin{array}{l}\text { Minimizing the maximum travel time in a combined model of facility location and network } \\
\text { design }\end{array}$ & $\begin{array}{l}\text { Contreras, I., Fernández, E. \& } \\
\text { Reinelt, G. }\end{array}$ & 2012 \\
\hline Improving access to health facilities in Nouna district, Burkina Faso & $\begin{array}{l}\text { Cocking, C., Flessa, S. \& } \\
\text { Reinelt, G. }\end{array}$ & 2012 \\
\hline $\begin{array}{l}\text { Modeling the budget-constrained dynamic uncapacitated facility location-network design } \\
\text { problem and solving it via two efficient heuristics: A case study of health care }\end{array}$ & Ghaderi, A. \& Jabalameli, M. & 2013 \\
\hline Facility location-network design problem: Reliability and investment budget constraint & $\begin{array}{l}\text { Shishebori, D., Jabalameli, M. } \\
\text { \& Jabbarzadeh, A. }\end{array}$ & 2014 \\
\hline A reliable budget-constrained fl/nd problem with unreliable facilities & $\begin{array}{l}\text { Shishebori, D. Snyder, L. \& } \\
\text { Jabalameli, M. }\end{array}$ & 2014 \\
\hline $\begin{array}{l}\text { Heuristic algorithms for solving an integrated dynamic center facility location - network } \\
\text { design model }\end{array}$ & Ghaderi, A. & 2015 \\
\hline $\begin{array}{l}\text { A combined facility location and network design model with multi-type of capacitated } \\
\text { links and backup facility and non-deterministic demand by fuzzy logic }\end{array}$ & $\begin{array}{l}\text { Asl, A., Zarandi, F. \& Sadeghi, } \\
\text { A. }\end{array}$ & 2016 \\
\hline Sustainable urban facility location: Minimising noise pollution and network congestion & $\begin{array}{l}\text { Hammad, A. Akbarnezhad, A. } \\
\text { \& Rey, D. }\end{array}$ & 2017 \\
\hline $\begin{array}{l}\text { An integrated network design and scheduling problem for network recovery and } \\
\text { emergency response }\end{array}$ & Iloglu, S. \& Albert, L. & 2017 \\
\hline $\begin{array}{l}\text { A Lagrangian relaxation approach to fuzzy robust multi-objective facility location network } \\
\text { design problem }\end{array}$ & $\begin{array}{l}\text { Shishebori, D., Babadi, A. \& } \\
\text { Noormohammadzadeh, Z. }\end{array}$ & 2018 \\
\hline Facility location for recovering systems of interdependent networks & $\begin{array}{l}\text { Mooney, E., Almoghathawi, Y. } \\
\text { \& Barker, K. }\end{array}$ & 2019 \\
\hline Integrating location and network restoration decisions in relief networks under uncertainty & Sanci, E \& Daskin, M. & 2019 \\
\hline Regional logistics network design in mitigating truck flow-caused congestion problems & $\begin{array}{l}\text { Gan, M., Li, X., Zhang, F. \& } \\
\text { He, Z. }\end{array}$ & 2020 \\
\hline $\begin{array}{l}\text { Combined facility location and distribution network design problem: Progressive models } \\
\text { and a case study }\end{array}$ & Kucukkoc, I. \& Acar, M. & 2020 \\
\hline $\begin{array}{l}\text { A stochastic programming model with endogenous and exogenous uncertainty for reliable } \\
\text { network design under random disruption }\end{array}$ & $\begin{array}{l}\text { Bhuiyan, T., Medal, H. \& } \\
\text { Harun, S. }\end{array}$ & 2020 \\
\hline $\begin{array}{l}\text { Multidimensional resource allocation for freight transportation project planning and } \\
\text { decision making }\end{array}$ & $\begin{array}{l}\text { Bhattarai, S., Golias, M., } \\
\text { Mishra, S. \& Talebian, A. }\end{array}$ & 2020 \\
\hline $\begin{array}{l}\text { Exact robust solutions for the combined facility location and network design problem in } \\
\text { hazardous materials transportation }\end{array}$ & Liu, X. \& Kwon, C. & 2020 \\
\hline $\begin{array}{l}\text { A fuzzy capacitated facility location-network design model: A hybrid firefly and invasive } \\
\text { weed optimization (FIWO) solution }\end{array}$ & $\begin{array}{l}\text { Asl, A., Zarandi, F., Sotudian, } \\
\text { S. \& Amini, A. }\end{array}$ & 2020 \\
\hline $\begin{array}{l}\text { Design of a location and transportation optimization model including quality of service } \\
\text { using constrained multinomial logit }\end{array}$ & $\begin{array}{l}\text { López-Ospina, H., Agudelo- } \\
\text { Bernal, A., Reyes-Muñoz, L. } \\
\text { Zambrano-Rey, G. \& Pérez, J. }\end{array}$ & 2021 \\
\hline $\begin{array}{l}\text { An integer L-shaped algorithm for the integrated location and network restoration problem } \\
\text { in disaster relief }\end{array}$ & Sanci, E. \& Daskin, M. & 2021 \\
\hline Optimizing locations of waste transfer stations in rural areas & $\begin{array}{l}\text { Chen, Y., Lai, Z., Wang, Z., } \\
\text { Yang, D. \& Wu, L. }\end{array}$ & 2021 \\
\hline
\end{tabular}

